
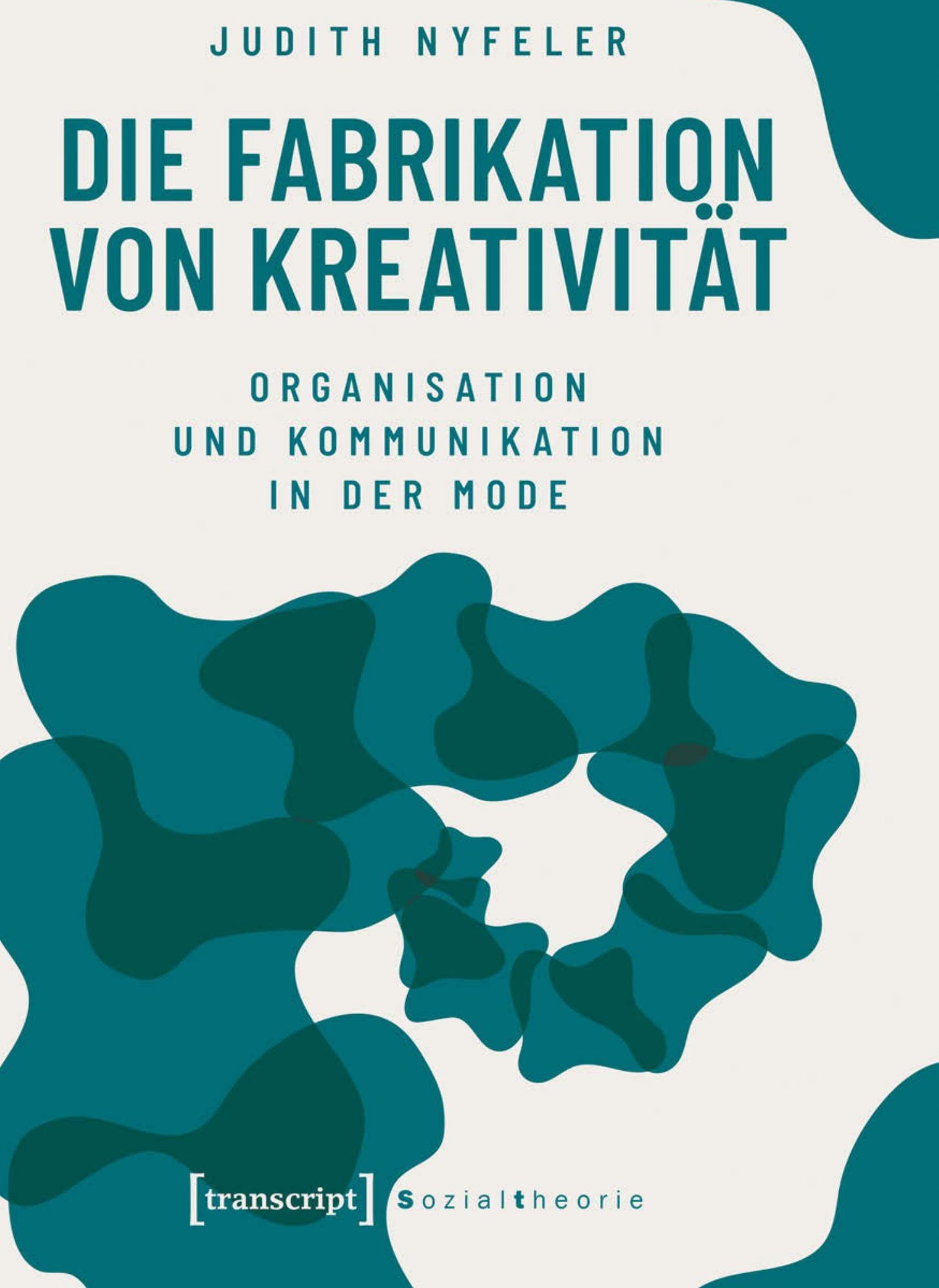
Judith Nyfeler

Die Fabrikation von Kreativität 
Fashion is a form of ugliness so intolerable that we have to alter it every six months. (Oscar Wilde)

Judith Nyfeler promovierte 2018 an der Universität Luzern. Sie lehrt und forscht im Bereich Organisations- und Wirtschaftssoziologie. Ihre Themenschwerpunkte sind Kreativität, Sprache und Mode. 
Judith Nyfeler

\section{Die Fabrikation von Kreativität}

Organisation und Kommunikation in der Mode 
Die vorliegende Arbeit wurde 2018 vom Soziologischen Seminar der Kulturund Sozialwissenschaftlichen Fakultät der Universität Luzern als Dissertation angenommen.

Gutachter*in: Prof. Dr. Raimund Hasse und Prof. Dr. Sophie Mützel

Publiziert mit Unterstützung des Schweizerischen Nationalfonds zur Förderung der wissenschaftlichen Forschung

\section{Bibliografische Information der Deutschen Nationalbibliothek}

Die Deutsche Nationalbibliothek verzeichnet diese Publikation in der Deutschen Nationalbibliografie; detaillierte bibliografische Daten sind im Internet über http://dnb.d-nb.de abrufbar.

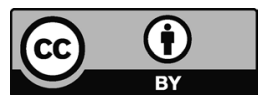

Dieses Werk ist lizenziert unter der Creative Commons Attribution 4.0 Lizenz (BY). Diese Lizenz erlaubt unter Voraussetzung der Namensnennung des Urhebers die Bearbeitung, Vervielfältigung und Verbreitung des Materials in jedem Format oder Medium für beliebige Zwecke, auch kommerziell. (Lizenztext:

https://creativecommons.org/licenses/by/4.o/deed.de)

Die Bedingungen der Creative-Commons-Lizenz gelten nur für Originalmaterial. Die Wiederverwendung von Material aus anderen Quellen (gekennzeichnet mit Quellenangabe) wie z.B. Schaubilder, Abbildungen, Fotos und Textauszüge erfordert ggf. weitere Nutzungsgenehmigungen durch den jeweiligen Rechteinhaber.

Erschienen 2019 im transcript Verlag, Bielefeld

(C) Judith Nyfeler

Umschlaggestaltung: Maria Arndt, Bielefeld

Korrektorat: Dr. Wolfgang Delseit, Köln

Satz: Isabel Lina Christen, Basel

Druck: Majuskel Medienproduktion GmbH, Wetzlar

Print-ISBN 978-3-8376-4992-5

PDF-ISBN 978-3-8394-4992-9

https://doi.org/10.14361/9783839449929

Gedruckt auf alterungsbeständigem Papier mit chlorfrei gebleichtem Zellstoff. Besuchen Sie uns im Internet: https://www.transcript-verlag.de

Unsere aktuelle Vorschau finden Sie unter

www.transcript-verlag.de/vorschau-download 


\section{Inhaltsverzeichnis}

\section{Abbildungs- und Tabellenverzeichnis | 7}

\section{$1 \quad$ Einleitung | 9}

\section{Forschungsdesign | 23}

2.I Standorte $\mid 24$

2.2 Techniken $\mid 27$

2.3 Analyse oder was das Feldmaterial erzählt | 35

$3 \quad$ Zur Feldstrukturierung der Creative Industries | 37

3.I Zum Feldbegriff der Creative Industries 39

3.2 Empirische Feldbeschreibungen 49

3.3 Der Modesektor 56

3.4 Fazit I: Der Creative Director im Fokus $\mid 63$

4 Ansätze der Kreativitätsforschung | 65

4.I Psychologische Anfänge: Charakter und Fähigkeit | 66

4.2 Sozialtheoretische Weiterentwicklungen $\mid 72$

$5 \quad$ Kreativität als Gegenstand der Organisationsforschung $\mid 79$

5.I Kreativität zwischen Organisationen $\mid 80$

5.2 Kreativität innerhalb der Organisation $\mid 88$

5.3 Fazit II: Kreativität als Resultat mehrlagiger Spannungsverhältnisse | 93

6 Die Organisation der Modekreation | 95

6.I Verlässlichkeit und Variation: Routineforschung | 96

6.2 Öffnung und Schließung: Kontingenztheorie | Io6

6.4 Technologische Aspekte und die Neuheitsroutine I46 


\section{Darstellungsformen der Mode $\mid$ I53}

7.I Sprache und Kreativität | I55

7.2 Orientierende Narrative $\mid \mathrm{I} 60$

7.3 Inszenierende Shows $\mid$ I70

7.4 Finalisierendes Storytelling | I78

7.5 Kommunikative Aspekte, Narrative und Zeremonien | I85

8 Organisierte Kreativität in der Mode $\mid$ I93

8.I Das Prinzip des Kreierens neuer Moden $\mid$ I96

8.2 Lose Kopplung von Technologie und Sprache $\mid$ I99

8.3 Schlussbemerkungen und Ausblick | 2II

Bibliografie | 2I5

Anhänge | 239

Anhang A: Übersicht Interviews und Beobachtungsprotokolle | 239

Anhang B: Organisation und Darstellung der Modekreation | 24I

Anhang C: Interviewleitfaden $\mid 242$

Anhang D: Vorlage Feldnotizen | 243

Anhang E: Telefoninterviews | 244

Dank | 245 


\section{Abbildungs- und Tabellenverzeichnis}

\section{AbbiLdungsVerzeichnis}

Abb. I: Zentrale Kategorien der Kreativität: Beschreibungen, Definitionen, repräsentative Studien, eigene Darstellung nach Slavich/Svejenova (20I6: 24I) Abb. 2: Die innovative Organisation »Adhocracy« nach Mintzberg (I979: 198) Abb. 3: Organisation der Modekreation (I): Von der Vorstellung der Entwürfe bis zum Muster Abb. 4: Organisation der Modekreation (2): Vom Muster zum Kollektionsmuster Abb. 5: Kampagnenbild »On the Road« Abb. 6: Kampagnenbild »Instrumente« Abb. 7: Skizze der Kollektionsmodelle und Berufe im Hauptgebäude Abb. 8: Kampagnenbild »La Route de la Soie« Abb. 9: Kollektionslogo Abb. Io: Bildausschnitt des Fotoshootings im spanischen Klublokal Abb. II: Kampagnenbilder mit Comic-Elementen Abb. I2: Präparierte Schuhe für die Modenschau Abb. I3: Beispiel der Postproduktion für den Newsletter Abb. I4: Argentinisches Gedicht aus einer Broschüre Abb. I5: Generelle Aspekte des Entwurfs Abb. I6: Generelle Aspekte der Inszenierung (I) Abb. I7: Spezifische Aspekte des Fotoshootings/der Modenschau (2) Abb. I8: Spezifische Aspekte der Postproduktion/Marketing (3)

\section{TABELLENVERZEICHNIS}

Tab. I: Statistische Informationen und Verschiedenheiten Tab. 2: Liste des empirischen Feldmaterials 



\section{$1 \quad$ Einleitung}

Seit I868 definierte und kontrollierte die »Chambre Syndicale de la Haute Couture « aus der französischen Hauptstadt die Modetrends (Lipovetsky 2002; Crane 20I2). Es entstand ein Modediktat, das weltweit zum Vorbild genommen wurde. Die französische Modekammer sollte die Hegemonie der Modeherstellung schützen und deren Monopol in Paris erhalten. Haute Couture ist bis heute eine regulierte und überwachte Bezeichnung, welcher (aus finanziellen Gründen) nur noch wenige Modehäuser nachkommen. ${ }^{\mathrm{I}}$ Mit den technologischen Entwicklungen serieller Herstellung von Konfektionsbekleidung haben sich Designer und ihre >Häuser rund um den Globus etablieren können, weshalb die Kammer sich I973 in die »Fédération Française de la Couture, du Prêt-à-porter des Couturiers et des Créateurs de Mode « umbenannte. ${ }^{2}$ Neben das ehemalige Modezentrum Paris sind nun weitere getreten und so gelten auch London, Mailand, Kopenhagen, Stockholm, New York oder Hongkong als Modestädte, in denen Modewochen stattfinden und in denen sich berühmte Designer und Stammhäuser niedergelassen haben (Kawamura 2005; Aspers 20I0).

Dadurch ist die Kontrolle über die Mode dezentralisiert worden. Längst ist nicht mehr nur die französische Modekammer für die saisonale Orchestrierung neuer Trends zuständig. Eine Vielzahl von Akteuren der Modeindustrie, von Unternehmen, über deren Angestellte bis hin zu den im Jargon auch mit »Influencer« betitelten Modebloggern oder Trendagenturen beeinflussen Marotten und Moden und werden gleichzeitig davon beeinflusst (Taylor 2005; Mora 2006; Skov 2006; Giertz-Mårtenson 2008). Es entstehen wechselseitige Beziehungen zwischen Beobachtern und Beobachteten, die typisch für dy-

1 Haute Couture ist ein stark regulierter Begriff. Eine der Regeln war, dass mindestens zweimal jährlich eine Kollektion mit vorgeschriebener Anzahl Kleidern inklusive eines Brautkleides in den Räumlichkeiten des in Paris liegenden Hauptsitzes der Firma an lebendigen Models vorgestellt wird (vgl. Crane 2012; Djelic/Ainamo 1999).

2 | Im Folgenden wird aus Gründen der besseren Lesbarkeit ausschließlich die männliche Form benutzt. Es sind dabei aber sowohl männliche als auch weibliche Personen gemeint. 
namische und innovative Marktlandschaften wie die Mode sind: Modehäuser sind von Aktualitäten des Weltgeschehens betroffen, weshalb die Relevanz und Brisanz dieser Geschehnisse nicht selten zur Inspirationsquelle für Designer und deren Häuser werden. Infolgedessen dienen soziale Inspirationsquellen dem Modedesign als Grundlage künstlerischen Schaffens (Esposito 20II; Aspers/Godart 2013).

Aufgrund dieser wechselseitigen Beeinflussung, Beobachtung und Nachahmung verschwimmt die Trennlinie zwischen der Inspirationsquelle und dem eigenen Beitrag. Vor diesem Hintergrund kann ein nicht ausgewiesenes, fremdes geistiges Eigentum in Form einer Imitation, Kopie oder Fälschung zum Problem juristischer Grenzziehung werden. Dieses juristische Problem, das sich aufgrund der Flüchtigkeit der Mode oftmals von selbst entschärft, bevor es überhaupt zu einer Klage kommt, zeigt sich andererseits als Demokratisierung unbezahlbarer Modetrends: das stilgerechte und detailgetreue Nachmachen von Designoriginalen. Erst kürzlich hat sich ein wissenschaftliches Spezialistentum herausgebildet, das sich auf Probleme der Hoheitsrechte geistigen Eigentums in den Creative Industries versteht (Raustiala/Sprigman 2006; McRobbie 20I6). Unternehmen intervenieren selten und nur in ökonomisch lukrativen Fällen juristisch, denn Kreativen schmeichelt es, wenn »ihre« Idee als Trend kopiert wird und dadurch allgemeine Verbreitung findet.

Die wechselseitige Beobachtung führt dazu, dass bestimmte Muster und Moden feststellbar sind. Je kohärenter und abgestimmter die an den Modewochen vorgeführten Modekollektionen der internationalen Designergarde, desto deutlicher der scheinbare Trend, der ein Jahr später auch die günstigen Bekleidungsketten erreicht haben wird, und desto klarer der Ausweis der gegenseitigen Wahrnehmung (Blumer 1969; Godart/Mears 2009; Godart/Shipilov/ Claes 20I4). ${ }^{3}$ Dies legt die Vermutung nahe, dass Unsicherheiten, Ambivalenzen und das Risiko von kreativen Fehlschlägen durch die Orientierung am Vorbild des Modediktats der Großen reduziert werden. Das bedeutet, dass man kaum etwas falsch machen kann - außer, zu langsam zu sein und den Trend zu verpassen.

Während diese Grundannahme zum Modesystem weit verbreitet ist, beleuchtet die vorliegende Studie einen anderen Aspekt der Kreativitätshervorbringung. Diese Arbeit argumentiert, dass Modeunternehmen zwar Bezug auf Vorbilder nehmen und dadurch einen Referenzrahmen der Hervorbringung

3 | Nicht nur feldkonfigurierende Ereignisse wie Modenschauen oder Messen dienen als mögliche Inspirationsgrundlage, sondern auch geteilte Produktionsinfrastrukturen. Als Plattformen gegenseitiger Wahrnehmung findet dort die Produktion unterschiedlicher Modekollektionen nur wenige Meter voneinander statt. Räumliche Nähe in Produktionsstätten vereinfacht das Kopieren und Abschauen neuer Designs, bevor sie überhaupt die Öffentlichkeit erreicht haben. 
von Kreationen schaffen, den Prozess der Kreativitätshervorbringung aber vornehmlich technologisch organisieren, indem sie sich an Technologien halten, die sie beherrschen und an bestätigten Maßstäben und Modellen orientieren. Damit schützen sie die Operationen vor Umwelteinfluss und betreiben die kontinuierliche Produktionstätigkeit auf effiziente Weise. Diese Form der Versicherungstechnik fungiert als Mechanismus der Unsicherheitsreduktion, denn durch das Organisieren des Kreationsprozesses werden Unsicherheiten zwischen Designarbeiten und Resultat, die auch bei kleineren Transitionsaufgaben entstehen können, verringert und Variationen kontrolliert. Vor diesem Hintergrund haben Organisationen die Möglichkeit, Routinen und Projekte zu managen und die Kreationen in begrenztem, kontrolliertem Maße zu variieren. Dies ist wichtig, da Mode ein Termingeschäft ist und auf Zeit angelegt ist, d. h., nur wenig Zeit für die anschließende Verbreitung einer Neuerung zur Verfügung steht.

Kreativität und Innovation werden im Alltagsverständnis oft in einem Atemzug verwendet. Manchmal wird Innovation auch als Ergebnis vorangehender kreativer Prozesse verstanden (Woodman/Sawyer/Griffin I993; Carlsen/Clegg/Gjersvik 20I3; Cronin/Loewenstein 20I8). Dabei gilt es zu betonen, dass der kreative Prozess nicht zwingend zu neuen Kreationen führen muss (Shalley/Gilson 20I7). Während kreative Prozesse in einer Vielzahl von organisationalen Aktivitäten eine Rolle spielen - ein Grund dafür, weshalb die Organisationsforschung ein explizites Interesse an der Kreativitätsforschung zeigt -, fällt auf, dass diese Form der alltäglichen Kreativität nicht unbedingt zu kreativen Produkten, Neuerungen oder Innovationen führt. Cronin und Loewenstein (2018) verstehen den Kreativprozess als Handwerk, das aus drei unterschiedlichen kreativen Produkten besteht: erstens der Einsicht, zweitens einer Erfindung und drittens der Erleuchtung, die bestehende Denkweisen fundamental verändert. Kreativität wird deshalb oft als Entstehungsprozess später implementierter Innovationen verstanden (Shalley/Gilson 20I7: 607). ${ }^{4}$ Deshalb lohnt es sich, an dieser Stelle auf das Verhältnis von Kreativität und Innovation einzugehen. Während die Innovationsforschung viel Vorarbeit geleistet hat und für die Kreativitätsforschung anschlussfähig war, so unterscheiden sich diese beiden Begriffe dennoch wesentlich. Innovationen setzen Diffusion voraus (Rogers 2003), bergen ein vergleichsweise hohes Risiko des Scheiterns, sind schwer zu planen und nicht selten sind sie mit instrumentellen Ansprüchen verbunden - also versprechen eine Verbesserung -, während sich Kreati-

4 | Shalley und Gilson (2017) wenden die graduelle Eigenschaft von Innovationen (radikal bis inkrementell) direkt auf die Kreativität an und stellen damit nicht nur eine terminologische Verbindung, sondern auch eine konzeptuelle Verwandtschaft her. 
vität schlicht und einfach vom Bekannten abheben und dieses erneuern soll. ${ }^{5}$ Ebenso kennzeichnend ist, dass nicht alle kreativen Versuche weiterverfolgt werden. Für den hier vorliegenden Fall der Mode gilt, dass Innovationen keine Seltenheit, aber auch nicht die Regel sind (Crane 2000). Technologische Innovationen können z. B. in Form technischer Textilien, Färbtechniken oder Produktionstechnologien vorkommen, die von Modefirmen adaptiert und im Modedesign eingesetzt werden. Modische Innovationen mit Langzeitwirkung, so wird hier argumentiert, sind zwar möglich, aber deshalb selten, weil Mode auf Zeit angelegt ist und aufgrund des schnellen Turnus oft kaum Zeit für die anschließende Diffusion bleibt bzw. die Verbreitung der Neuerung während der Entwicklung zweitrangig ist. In der Kreativitäts- und Modeforschung wird deshalb oft von »ästhetischen" (McRobbie 20I6) oder »stilistischen Innovationen « gesprochen (Cappetta/Cillo/Ponti 2006). ${ }^{6}$ Bohn bezeichnet Mode als Temporalphänomen - im Vergleich zu Kleidung, die ein Universalphänomen ist: 7

Das Spezifische - und am Beginn der Moderne noch Überraschende - der Mode besteht offenbar darin, dass sie sich Geltung verschafft, obwohl sie schon im Aufscheinen ihr Verschwinden mitverkündet. Mode wird gerade deshalb zu einer typischen Form der Kontingenzbewältigung, die das Kontingente, Willkürliche, Kapriziöse akzeptabel macht, da sie dessen Untergang und Vergänglichkeit gleich mitkommuniziert. (Ebd.: 119)

Die Kurzfristigkeit und Temporalität werden vor allem in den saisonalen Kollektionen deutlich, die - kaum im Verkauf - wieder überholt und »old-fashioned« sind (Kawamura 2005; Aspers 20I0). In dieser Vergänglichkeit und dem

5 | Diffusion fungiert dann als Bekanntheitsmesser im institutionellen Feld, d. h., je weniger diffundiert eine Mode, desto eher wird von avant-garde gesprochen. Wird einem weit verbreiteten Trend nicht Folge geleistet, muss das Argument stark sein, das die Ablehnung legitimiert, um nicht als altmodisch zu gelten.

6 | Gemäß Cappetta, Cillo und Ponti (2006) bewegen sich stilistische Innovationen zwischen inkrementeller Veränderung und radikaler, "treibender Gärung“. Mit der Zeit stellt sich ein Konvergenzpunkt ein - die Innovationsforschung würde diesen mit erfolgreicher Diffusion bezeichnen -, an dem die Neuerung von den meisten Modehäusern als Referenzpunkt verwendet wird. Die Autoren unterscheiden dabei nicht zwischen innovativen und kreativen Neuerungen, d. h., es kann sowohl an einen Modetrend (kreative Modifizierung) als auch an eine dominante technische Veränderung (technologische Innovation) gedacht werden.

7 | Ferner unterscheidet Simmel (1986: 182) zwischen Kleidung, die von angepasster Zweckmäßigkeit bestimmt ist, und Mode, die sich frei von solcherlei Entscheidungen formt. 
steten Wandel sieht Blumer das Potenziale der Mode, die - eine »moving and changing world « vorausgesetzt - die Freiheit in sich trägt und das Handeln von alten Beschränkungen befreit, indem sie ermöglicht, neue Richtungen einzuschlagen (Blumer I969: 289). ${ }^{8}$ Während modische Ausgestaltungen - die Moden - auf Zeit angelegt und ephemer sind, ist die Mode als Faktum unvergänglich (Simmel I986: 204). Sie ist somit nicht nur eine Entwicklungs- und Produktions-, sondern auch eine Zeiteinheit (Patriotta 2003: 356). Zeitlichkeit beschreibt deshalb einen wichtigen Aspekt der Mode.

Für die Organisationsforschung ist der Fall der Mode deshalb spannend, weil ständig und verlässlich, in einem regelmäßig erneuernden Zyklus, neue Kreationen hervorgebracht und rigide zeitliche Vorgaben eingehalten werden. ${ }^{9}$ »Es entsteht nicht irgendwo ein Artikel, der dann Mode wird, sondern es werden Artikel zu dem Zweck aufgebracht, Mode zu werden. In gewissen Zeitintervallen wird eine neue Mode $a$ priori gefordert, und nun gibt es Erfinder und Industrien, die ausschließlich an der Ausfüllung dieses Rahmens arbeiten«, schreibt Simmel (I986: I83) und meint damit die Verschiebung der ehemals personalen Ideengenese zu einer kommerziell geprägten Wiederholungswirtschaft. Mode ist die Institution, die sich mittels vestimentärer Gestaltung und organisatorischer Prozesse regelmäßig aktualisiert (Barthes I986). Dadurch unterscheidet sie sich von anderen Innovationsfeldern. Als Konsequenz dieser Herausforderung muss die Möglichkeit der routinierten Planung, Koordination und Verwaltung unterstellt werden. Insofern fallen nicht nur Terminierungsaspekte, sondern auch Formen der Netzwerkbildung, des Wettbewerbs und der kooperativen Zusammenarbeit sowie Legitimität, Sprache und Kom-

8 | Blumer (1969: 290) versteht Mode als eine Funktion, die Ordnung herstellt: "Fashion should be recognized as a central mechanism in forming social order in a modern type of world, a mechanism whose operations will increase."

9 | Die sozialwissenschaftliche Modeforschung erklärt Wandel auf unterschiedliche Weise: mit Klassenmoden, die Schichten durchdringen, adaptiert und revolutioniert werden und zum Paradox zwischen Distinktion und Konformität führen (Simmel 2013); mit der mit Neid betrachteten Auszeichnung, die im ostentativen Konsum und Verschwendung zur Schau gestellt wird (Veblen 1986); mit der Konkurrenz des kapitalistischen Marktes, dem Gefälle zwischen Zentrum (Paris) und Peripherie, der Rolle der Modeschöpfer und Konfektionshäuser oder der Dynamik der Materialien (Sombart 1986); mit dem Willen, Güter, Reichtum und Prestige eigens durch Wegwerfen und Neuaneignungen übertreffen zu können (Barthes 1986); oder mit Störungen im Modezyklus, die als Mechanismus für Produktivitätswachstum und wirtschaftlichen Fortschritt verstanden werden können, denn nur aufgrund der Standardisierung ist die periodische, strukturelle Liquidierung vergangener Fehler in der Mode überhaupt möglich, das in Anschluss als "refreshing mechanism " zu neuen Ideen und Wandel führt (Chai/Earl/Potts 2011). 
munikation als zentrale Themen auf. Daraus folgernd versteht diese Arbeit die Organisation der Modekreation kollektiv (d. h., sie wird durch eine Vielzahl von Akteuren initiiert und umgesetzt), organisiert (d. h., die Koordination erfolgt über Projekte, bei denen bestimmte Organisationen bzw. Abteilungen oder Stellen federführend sind) und institutionell (d. h. durch Werte und Grundüberzeugungen so abgesichert, dass sie erwartet wird) geprägt. Kreativität ist deshalb nicht das verblüffende Resultat eines einzelnen »Genies«. Der institutionellen Dimension von Kreativität, die sowohl die Position im Team als auch die vorgängig installierten, unhinterfragten Vorstellungen und Erwartungen an die Organisation bzw. die organisationale Rolle der Kreativen umfasst, wurde von der organisationstheoretischen Kreativitätsforschung bislang kaum beachtet (Bouty/Gomez 20I6: 233). Zusammenfassend sehen sich Modefirmen damit konfrontiert, Ambivalenzen wie das Verhältnis von Variation und Verlässlichkeit, Tradition und Erneuerung sowie Kommerz und Kulturproduktion organisatorisch zu bewerkstelligen (Gilson u. a. 2005; Tschang 2007; Caves 2012).

Die Wiederholungswirtschaft hat zu Rationalisierung und Standardisierung im Feld der Mode geführt, die heute viele Formen neuer Modekreationen bedingen. Dies lässt die Frage offen, was an der geregelten Temporalität und den Auswüchsen zyklischer Wiederholungen überhaupt noch originell, neu und kreativ ist. Deshalb lautet die Fragestellung in dieser Arbeit:

- Wie wird Kreativität in der Modebranche hervorgebracht?

- Was macht die erfolgreiche Kommunikation der Neuerungen wahrscheinlich?

- Welche Organisationsformen und Bedingungen spielen eine Rolle im Entwicklungsprozess und welche Akteure sind daran beteiligt?

Die Lücke, die diese Arbeit zu füllen versucht, widmet sich der Frage, wie trotz standardisierter, serieller Herstellung von Neuerungen dennoch Kreativität reklamiert wird. Für die Creative Industries gilt generell, dass das künstlerische Sentiment und der schöpferische Grundwert der Kreativität mit denjenigen der effizienten Verfahrensweisen und Produktionsaktivitäten unvereinbar sind (Suddaby u. a. 20I7). Mode wird üblicherweise jedoch nicht zu den Creative Industries gezählt und findet nur selten Erwähnung als solche. Dennoch scheint Kreativität in der Mode fern von Rechenschaftspflicht und Erklärungsbedarf $\mathrm{zu}$ bestehen, was teilweise auf die Selbstbeschreibung der Creative Industries zurückgeführt werden kann. Was im Feld der Mode entsteht, hat per se eine kreative Wirkung. Diese generellen Annahmen erklären Entscheidungen und Handlungen während der Entwicklungsphase, d. h. im Vorfeld der Produktion kreativer Güter, sowie während der Kreativitätshervorbringung. 
Das empirische Feldmaterial hat gezeigt, das der gesellschaftliche Glaube besteht, dass technologische und wiederholte Vorgänge kein legitimes Argument für Kreativität sind und kreativer Erfolg auf weitaus mehr als guter Organisation und technischer Kontrolle der Produktionskapazitäten beruht. Das lässt sich daran erkennen, dass Modefirmen ihre Kollektionen ressourcenintensiv und ostensiv inszenieren. Deshalb fügt diese Arbeit der kollektiven und organisationalen Auffassung von Kreativität eine institutionelle hinzu: Um erfolgreich modisch und kreativ zu sein, muss die Kreation bestehende soziale Werte und Vorstellungen beachten, d. h., normative Abgleichung oder moralische Passung demonstrieren. Werte und Vorstellungen sind institutionelle Erwartungen, an denen sich Modefirmen ausrichten und die sie in ihre Formalstruktur überführen. Damit versuchen sie, den extern postulierten Gesellschaftsansprüchen gerecht zu werden. Die formale Angleichung und Passung führt dazu, dass ein Produkt, eine Person oder eine Organisation als legitim bewertet wird (DiMaggio/Powell I983; Suchman I995; Suddaby u. a. 20I7). ${ }^{\text {10 }}$ Normalerweise werden Designs zu neuen Kreationen kombiniert, modifiziert oder ergänzt. Diese Praxis der modischen Feinabstimmung ist die Vorgehensweise, derer sich die meisten Modefirmen bedienen. So können Modefirmen trotz Erneuerung auf radikale Brüche verzichten und trotzdem modisch erscheinen. Hierbei wirken Sprachspiele und zeremonielle Aktivitäten konstitutiv und »kreativisierend « und heben den Neuheitswert und die individuelle Gestaltung der Neuerung hervor. Diese Hervorbringung modischer Neuerungen ist jedoch relativ voraussetzungsvoll, denn erstens muss eine Vorstellung davon bestehen, was als neu und kreativ erachtet wird, und zweitens muss diese Vorstellung auf modische Weise umgesetzt werden. Etablierte Modefirmen haben an dieser Stelle einen Vorteil, denn sie können das Wissen über existierende Moden gewinnbringend einsetzen (Cappetta/Cillo/Ponti 2006: I285). Deshalb kann die Grundannahme formuliert werden, dass Kreativität - und so auch ihre modischen Ausgestaltungen - höchst selten ex nihilo entsteht.

Diese Studie bestärkt die These, dass Kreativität eine Zuschreibung ist, die dann erfolgt, wenn soziale Akteure neue Kreationen kommunikativ als besonders ausweisen - trotz der Wiederholung und dem Befolgen bestimmter technologischer Bedingungen und Verfahren. Kreativität ist weder begründungspflichtig noch eine Rechtfertigungskategorie (beispielsweise für Ladenpreise),

10 | Am wenigsten Legitimität - und Kreativität - erhält die Praxis des Kopierens geistigen Eigentums, denn sie wird als moralisch unpassend wahrgenommen und ist somit nicht legitim. Am meisten Legitimität und die Zuschreibung von Kreativität erhalten Neuentwicklungen, die zwar als sehr avantgardistische, exzentrische und weil stark abweichend von bestehenden Normen als exotische Kreationen abgetan werden, jedoch der Neuheitsgehalt am größten ist. Haute Couture wird deshalb oft als Kunstform bezeichnet. 
denn die Zuschreibung besteht temporär und ihr Erhalt ist nicht garantiert. Deshalb bestehen bestimmte organisationale Formen lediglich für >Kreativitätszwecke<, so z. B. die Bestimmung eines Kollektionsthemas, besondere Projektkooperationen mit renommierten Kunstschaffenden oder bekannten Models, sowie Modenschauen und Fotoshootings, durch welche die Zuschreibung ständig erneuert und aufrechterhalten werden kann.

Als Ausgangspunkt für die theoretischen Überlegungen wurden zwei Schweizer Modebetriebe untersucht, wobei der Forschungsschwerpunkt auf den alltäglichen Tätigkeiten der Kollektionshervorbringung im Unternehmen lag. Um festzustellen, wie Kreativität im Modedesign generiert und entwickelt wird, musste in erster Linie untersucht werden, was die Organisationen tun separat davon, was sie damit beabsichtigen. Diese Perspektive erschloss die analytische Trennung zwischen der Entwicklung (und Produktion) und der Kommunikation und Darstellung von Kreativität.

Einerseits, so wird hier kontingenztheoretisch argumentiert, produzieren Modefirmen auf der Basis von Routinen und Technologien, die sich zu einer modularen Aktivitätsstruktur zusammenstellen. Organisationen der Creative Industries und folglich auch die des Modesektors trennen die Hinter- von der Vorderbühne, indem sie den organisationalen Betriebskern durch eine modulare Aktivitätsstruktur vor Unsicherheiten und Veränderungen der Umwelt abschotten. Damit dies gelingt, konzentriert man sich auf Fragen der technologischen Bedingungen der Produktion, und diese werden abgesondert und unabhängig von der Dynamik der Umwelt betrieben. Nur so kann die kontinuierliche Aktivität im Betriebskern gewährleistet werden. Dabei sind Rückgriffe auf Routinen vorherrschend, wodurch neue Kreationen auf spezifische Stile und Traditionen ausgerichtet werden. Basierend darauf werden die Möglichkeiten und das Maß an Kreativität zu einem wesentlichen Teil von Routinen und Technologien bestimmt. In der Kontingenzforschung werden unter dem Begriff Technologien sowohl technische Apparaturen, d. h. physische Ressourcen wie die Infrastruktur als auch nicht physische Ressourcen, wie das nötige und relevante Wissen, sowie Expertise und Erfahrung, gefasst (Child 1972; Thompson 2008; Mintzberg I989). Trotz der Bezeichnung des Kontingenzansatzes nach den aus der Umwelt stammenden »constraints and contingencies « (Thompson 2008: 24), - die deutsche Bezeichnung Situativer Ansatz verweist folglich mehr auf die situative Gestaltung der Organisation (Kieser 20I4) - ist der Mehrwert dieser Perspektive in der Abschottung des Organisationskerns von seiner Peripherie und Umwelt zu sehen. Durch das Puffern können interne Aktivitäten konstant bedient werden, während der externe Einfluss der Kontingenz auf den operativen Kern beschränkt wird. Organische, demokratische und teils dezentralisierte Strukturen prägen die innovative Organisation (Mintzberg 1989). Ebenso kennzeichnend ist, dass in Projekten wiederholt mit denselben Spezialisten kooperiert wird, so wie das auch für andere wirtschaft- 
liche Vernetzungen beschrieben worden ist. Da durch die Fluktuation der Projektteilnehmer die technologischen Möglichkeiten stark variieren, zahlen sich Wiederholungen der Projektkooperationen aus. Auf der Basis wechselnden Personals können innovative Organisationen in Projekten effektiv auf Veränderungen aus der Umwelt reagieren. Folglich werden Projekte als Etappenziele der Entwicklung und Produktion der neuen Kollektion verstanden, wozu sich permanente und temporäre Projektmitglieder zu Teams zusammenschließen. Durch die Verschränkung von temporären und auf Dauer angelegten Organisationsformen arbeitet diese Studie die Projektorganisation als grundlegende Form des Organisierens der Kreativitätshervorbringung heraus. Mithilfe der Kontingenzforschung wird die organisatorische Struktur von Kreationsprozessen in Modefirmen erläutert. In dieser Arbeit wird deshalb angenommen, dass die Organisation der Modekreation und die serielle Herstellung kreativer Güter auf den technologischen Aspekten der Kreativität beruhen. Die organisatorische Koordinierung von drei paradoxen Gleichzeitigkeiten, die da wären Verlässlichkeit und Variation, Schließung und Öffnung der Betriebsgrenzen und Permanenz und Wandel, werden in dieser Arbeit als Neuheitsroutine bezeichnet. Die Neuheitsroutine beschreibt den organisierten Prozess - die oben erwähnte Versicherungstechnik -, wie Organisationen sich an bewährte und beherrschte Technologien halten, Kontinuität herstellen sowie Unsicherheiten und Ambivalenzen reduzieren. Auf der Grundlage der genannten Prinzipien Routinen, Technologien und Projekte - können Modefirmen auf radikale Brüche verzichten und dennoch kreativ erscheinen. Hierdurch lassen sich Fehlschläge reduzieren und rigide zeitliche Vorgaben können eingehalten werden.

Andererseits, so wird hier auch neoinstitutionalistisch argumentiert, kann Kreativität als Phänomen der sozialen Wirklichkeit erst sprachlich und kommunikativ vermittelt wahrgenommen werden (Berger/Luckmann 20I3). Um die standardisierten Aktivitäten des täglichen Betriebs gegenüber den kollektiven Wertvorstellungen und Erwartungen aus der institutionellen Umwelt zu legitimieren, wird eine Formalstruktur der Organisation entworfen, die zeremoniell demonstriert und narrativ betont, dass die Organisation für die bestimmte Aufgabe geeignet ist und den Vorstellungen entsprechend operiert. Von Autoren wie Meyer und Rowan (1977) oder Bromley, Hwang und Powell (20I2) werden die Erwartungen auch als institutionelle Rationalitätsmythen bezeichnet.

Institutional rules function as myths which organizations incorporate, gaining legitimacy, resources, stability, and enhanced survival prospects. Organizations whose structures become isomorphic with the myths of the institu- tional environment - in contrast with those primarily structured by the demands of technical production and exchange - decrease internal coordination and control in order to maintain legitimacy. (Meyer/Rowan 1977: 340) 
Rational sind diese, weil sie die Regeln und Annahmen sozialer Ziele als auch die angemessenen Mittel und Zwecke festlegen; um Mythen handelt es sich, weil ihre Wirklichkeit und Wirksamkeit nicht einer »objektiven«, empirischen Überprüfung unterzogen werden können, sondern vielmehr auf dem geteilten Glauben daran gründen (Kieser/Walgenbach 20I0: 43). In Bezug auf die Kreativität beinhalten solche Mythen sowohl Personalisierungen, so z. B. den Werdegang oder den Verdienst charismatischer Personen, als auch »verkehrte« Vorstellungen kreativer Praxis, wie z. B. erwartete »Heureka«-Momente, dem freudigen Ausruf nach der Lösung eines schwierigen Problems. Hierbei werden Fehlversuche kaum erinnert. Die Mythen werden organisatorisch durch spezifische Strukturen, d. h. wenig Hierarchie und Formalität, sowie spezifisch für Kreativität eingerichtete Organisationssegmente (Creative Department) und Positionen (Creative Director) reproduziert. Insofern bewirken organisationale Prozesse eine Fortführung und tragen so zu Neuerungen bei (Hasse/Mützel/Nyfeler 20I9). Um auf die Umwelterwartungen zu antworten, errichten die Unternehmen (Legitimitäts-)Fassaden und implementieren zeremonienhaft Produktionsfaktoren (z. B. organisationale Abteilungen oder Professionen) unabhängig davon, ob sich diese messbar auf die Leistungserbringung auswirken oder die Effizienz steigern (Meyer/Rowan I977: 350 f.). Kreativität ist eine Basiskomponente der Formalstruktur von Organisationen der Creative Industries, die bei anderen Sektoren fehlt. Sie tangiert im Spezifischen die Formalstruktur, während die Aktivitätsstruktur einer >profanen Produktionsindustrie gleicht. »Formal structures that celebrate institutionalized myths differ from structures that act efficiently. Ceremonial activity is significant in relation to categorical rules, not in its concrete effects. [...] Activity, that is, has ritual significance: it maintains appearances and validates an organization« (ebd.: 355). Diese Formalstruktur schafft Vertrauen in die Organisation, deren Praktiken und Produkte (ebd.: 358 f.). Die Errichtung derartiger Kreativitätsfassaden (über-)betont dabei die Verwendung ungewohnter Herangehensweisen und innovativer Resultate und demonstriert die individuelle Verschiedenheit im Referenzrahmen des Feldes (Khaire 20I4: 59). Die kreativen Idealisierungen werden fortan als Kreativitätszeremonien und Neuerungsnarrative bezeichnet. Diese Zeremonien und Narrative sollen einen kreativen Anschein erwecken und sind für die retrospektive Außendarstellung - aber auch zur Sinnstiftung - bedeutsam. Interessant hierbei ist, dass diese Narrative und Zeremonien jedoch bereits während des Prozesses der Hervorbringung von Neuerungen vorbereitet werden (z. B., indem bestimmte Kleider nur für die Modenschau produziert werden oder der Kollektionsentwicklung ein Arbeitstitel gegeben wird) oder gehen diesem sogar voraus (z. B. in Form von Inspiration, die zu Modellskizzen der Kollektion entworfen werden). Dazu zählen vorgängige Narrative, auf denen konstruierte Geschichten gründen und die der prospektiven Generierung von Sinn dienen, sowie inszenierte Ereignisse 
und schließlich das retrospektive Geschichtenerzählen der außengerichteten Kommunikation. Die Kreativitätszeremonien und Neuerungsnarrative haben deshalb weniger eine funktionale, denn eine legitimierende Bedeutung. Folglich ist eine Prämisse dieser Arbeit, Neuerungen im Modedesign als zeremonielle Aktivität zu verstehen, deren Narrative und Zeremonien zur Grundlage für Bewertungs- und Deutungspraktiken werden. Deshalb wird diese Diskussion unter dem Begriff der kommunikativen Aspekte der Kreativität geführt.

Indem die Bedeutung der Sprache neoinstitutionalistisch herausgearbeitet wird, schließt diese Arbeit an das Verständnis der Kontingenzforschung an, welches die enge Sichtweise des situativen Determinismus kritisiert und die re- und proaktive Einflussnahme auf situative Faktoren thematisiert (Child I972). Infolgedessen wird in dieser Arbeit die These aufgestellt, dass zu den technologischen Bedingungen der Entwicklung und Produktion kreativer Güter Fragen der Kommunikation und Darstellungen hinzukommen und damit die organisationale Kreativität hervorgebracht und bestätigt wird. Dadurch reagieren Organisationen auf Erwartungen der Umwelt und demonstrieren ihre institutionelle Passung. Das Argument schließt an die legitimatorische Wirkung zeremonieller Aktivitäten an, die »Konformität mit in der gesellschaftlichen Umwelt institutionalisierten Erwartungen« signalisieren und dadurch zur erhofften Kreativitätszuschreibung führen (Hasse/Krücken 2009: 239). Die institutionelle Passung wird mit institutionellen Vokabularen symbolisiert, wie beispielsweise mit Berufsbezeichnungen oder durch den Einsatz von spezifischem Fachjargon,II oder in zeremoniellen Inszenierungen, wie beispielsweise in Modenschauen oder Fotoshootings. Die institutionelle Passung wird mit der losen Kopplung von Aktivitäts- und Formalstruktur erreicht, die in Form der technologischen Herstellung und der kommunikativen Idealisierung aufeinandertreffen: »To maintain ceremonial conformity, organizations that reflect institutional rules tend to buffer their formal structures from the uncertainties of technical activities by becoming loosely coupled, building gaps between their formal structures and actual work (Meyer/Rowan 1977: 34I). Durch die lose Kopplung von und die Löcher zwischen Aktivitäts- und Formalstruktur schaffen sich Unternehmen die Möglichkeit, die Technologien unabhängig der Zeremonien bzw. der sprachlichen Darstellung zu bedienen, $d . h$. sowohl effizient und kontinuierlich als auch variativ und modisch zu produzieren. Alvarez u. a. (2005) bezeichnen die Balance zwischen kreativer Individualität und der Isomorphie aus dem Feld als »optimale Verschiedenheit«. Demzufolge wirft diese Arbeit erstens Licht auf die kollektive Hervorbringung und organisationale Koordinierung der Kreativitätshervorbringung und beleuchtet

11 | Wie z. B. High-End- oder High-Street-Mode oder made to order, d. h. individuell gefertigte Kleidung auf Maß, oder ready-to-wear, auch prêt-à-porter genannt, was handwerklich hergestellte Kleidung ab Stange, tragebereit, meint. 
zweitens die institutionellen Spannungen zwischen Kreativitätserwartungen und operativen Aktivitäten, die Organisationen mithilfe von Zeremonien und Narrativen kommunikativ regulieren. Diese unterscheiden das Feld der Mode von anderen Creative Industries sowie von sprofanen Produktionsindustrien. Die Frage zu stellen, wie Organisationen konkret Legitimität, produktive Kontinuität und Effizienz erzielen, ohne dabei die Grundsätze der Kreativität und Erneuerung zu beeinträchtigen, ist deshalb eine bis anhin unbeachtete theoretische Problemstellung mit wesentlichen empirischen Konsequenzen. Die Frequenz der kreativen Erneuerung in der Mode ist das Resultat der geschickten Verschränkung technologischer und kommunikativer Bedingungen, die es gilt, organisatorisch zu verwalten. Konzeptuell ergänzen sich die technologischen und die kommunikativen Aspekte zu dem, was von den Organisationen als Kreativität reklamiert und gesellschaftlich als solches anerkannt ist.

Diese Arbeit leistet einen Beitrag zur aktuellen Kreativitätsforschung und zur soziologischen Organisationsforschung. Insbesondere wird versucht, kontingenztheoretische und neoinstitutionalistische Annahmen zu kombinieren und dadurch eine neue Sichtweise auf das Problem der Kreativitätshervorbringung zu ermöglichen. Anhand der Kreativitätshervorbringung in der Modebranche werden die theoretischen und empirischen Auseinandersetzungen mit Kreativität in Organisationen weiter ausgeführt und als kollektives, organisationales und institutionelles Problem konzeptualisiert. Dabei wird auf kontingenztheoretische und neoinstitutionalistische Überlegungen zurückgegriffen. Insbesondere wird von der empirischen Erarbeitung des Themas erhofft zu verstehen, wie in der Modeindustrie Kreativität >entsteht<, soll heißen, welche Rolle ihr während des Entwicklungsprozesses zugedacht wird und welche Effekte dies für die Organisation neuer, modischer Kreationen hat.

Auch auf praktischer Ebene leistet diese Arbeit eine Erklärung für die standardisierte Kreativitätshervorbringung in Organisationen. Was sich in erster Linie als Demystifizierung eines unhinterfragten Sachverhaltes herausstellen mag, kann gleichermaßen zum Inhalt weiterführender personaler und organisationspolitischer Entscheidungen werden: Die Tendenz der Professionalisierung und Ökonomisierung, die sich in einer Vielzahl der Creative Industries abzeichnet, spiegelt sich auch im Fall der Modekreation. Wenn es früher noch um das künstlerische Erschaffen ging - die créateurs waren als Schnitt- oder Farbvirtuosen neuer Kleiderkreationen bekannt -, lässt sich aktuell eine Verschiebung der Profession des Creative Directors in Richtung des Projektmanagements, der Personalkoordinierung und der administrativen Verwaltung kreativer Aufgaben und Neuerungen beobachten. Trotz Rationalisierung ist die Standardisierung im Modesektor bislang eher schwach, denn obwohl sich die Entwicklung und Produktion effizient gestalten, gewähren sie interpretative und gestalterische Freiräume. 
Die Arbeit ist folgendermaßen aufgebaut: Zunächst wird im zweiten Kapitel das Forschungsdesign vorgestellt und das grundlegende Problem beim Zugang zum Feld expliziert, nämlich das stillschweigende Voraussetzen von Kreativität in Modebetrieben. Es hat sich schnell herausgestellt, dass Kreativität als Begriff im Alltagsgeschäft kaum vorkommt. Die Beschreibung des Forschungsvorhabens illustriert dies.

Das dritte Kapitel setzt sich mit dem Sektor der Mode auseinander. Dabei werden nach einer kurzen Beschreibung der wissenschaftlichen Diskussion rund um die Themen Kulturproduktion und Kreativität und dem Versuch, kreative Sektoren mit dem Feldbegriff des soziologischen Neoinstitutionalismus zu erläutern (3.I), verschiedene empirische Definitionen der Creative Industries vorgestellt (3.2), um eine für die Arbeit geeignete Definition des Modesektors und die branchentypischen Spezifitäten herauszuarbeiten (3.3). Dazu werden einerseits die in der organisations- und wirtschaftssoziologischen Literatur als notwendig erachteten Faktoren rezipiert, andererseits werden auch unterschiedliche Begrifflichkeiten der Kulturpolitik erfasst.

Ausgangspunkt für das vierte Kapitel ist die sozialwissenschaftliche Kreativitätsforschung, die sich seit Jahrzehnten mit Fragen der Kreativität beschäftigt. Das Kapitel konzentriert sich auf die Beschreibung der Entstehungsgeschichte, die in der Psychologie der I950er-Jahre gründet (4.I) und sich erst nach und nach zu einem sozialtheoretischen Problem entwickelt hat (4.2). Nach der interdisziplinären Auseinandersetzung und Ausarbeitung spezifischer fachlicher Unterschiede wird in Kapitel fünf Kreativität zum Thema der soziologischen Organisationsforschung gemacht. Darin wird weiter auf Kreativität zwischen Organisationen, z. B. als Resultat von Produktions- oder Innovationsnetzwerken (5.I), und auf Kreativität aus innerbetrieblichen Prozessen und Praktiken eingegangen (5.2). Dazu zählen beispielsweise Strukturen wie Führung, Hierarchie und Verfahren, die Kreativität ermöglichen oder hemmen.

In den nächsten zwei Kapiteln folgt eine theoriegeleitete Interpretation der Kreativitätshervorbringung. Das sechste Kapitel dient der Überprüfung der organisatorischen Aktivitäten der Entwicklung von Kreativität in Modefirmen. Dazu werden drei Organisationsformen analysiert - Routinen (6.I), Technologien (6.2) und Projekte (6.3) -, die jeweils prägnant konzeptuell präsentiert und anschließend mit empirischen Beispielen veranschaulicht werden. Das siebte Kapitel analysiert den Stellenwert institutioneller Zeremonien - Narrative (7.2), Inszenierungen (7.3.) und Storytelling (7.4) -, weshalb zunächst die Beziehung von Sprache und Kreativität geklärt wird.

Im achten abschließenden Kapitel wird die Frage aufgegriffen, inwiefern die Hervorbringung der Kreativität im Feld der Mode Bezüge zu kontingenztheoretischen und neoinstitutionalistischen Konzepten herstellen lässt. Die organisationale Kreativität in der Mode wird anhand der grundlegenden 
Prinzipien des Kreierens (8.I) und der losen Kopplung von Technologien und Sprache in der Mode (8.2) ausgeführt, wobei letztere die Gedanken zu technologischen Bedingungen und Fragen der Produktion (8.2.2) und der kommunikativen Voraussetzungen und Fragen der Darstellung (8.2.3) von Kreativität zusammenführt. Abschließend werden die zentralen Ergebnisse der Arbeit zusammengefasst (8.3). 


\section{Forschungsdesign}

Dieses Kapitel führt in die Vorgehensweisen und die Erhebungen ein, die zur Formulierung der Forschungsfrage und ihrer Beantwortung geführt haben. Das Kapitel listet die Quellen des Feldmaterials auf, skizziert die Techniken der Materialsammlung und deren Erzeugung und führt in die Methoden der Analyse ein. Die Analyse ist aus einem iterativen Prozess der Abduktion entstanden, der sich zwischen intensiven Feldphasen und konzeptuellen Erarbeitungen, empirischen Vertiefungen und theoretischen Diskursen hin und her bewegte (Swedberg 20I2). Zentral dabei war es, die sozialwissenschaftlichen Daten zu erforschen und diese mit eigenen Ideen und Einfällen zu kombinieren (ebd.: 3I). Die Besonderheit sozialwissenschaftlicher Forschungsfragen ist, dass sie sich auf Konzepte beziehen, die sowohl die theoretischen Erläuterungen begründen als auch Teil der alltäglichen Interaktionen und sozialen Konstruktionen sind, weshalb Konzepte nicht selten soziales Handeln gestalten (Sohlberg/Leiulfsrud 20I7). Der Kontext der Entdeckungen ist dabei von bedeutender Wichtigkeit, weil »empirical data should utlimately drive the theorizing process « (Swedberg 20I2: 7). ${ }^{12}$ Die bedeutende Qualität eines wissenschaftlichen, empirisch fundierten Berichts gründet deshalb auf der sorgfältigen und repetitiven Arbeit »from field to desk« und zurück (Czarniawska 20I4a).

Feldforschung favorisiert »Wie? Und warum?«-Fragen gegenüber den Formulierungen »Was? Und weshalb?« Besuche an den Plätzen des Geschehens bieten die Möglichkeit für sowohl exploratives als auch explanatives Forschen, besonders in Feldern, die neu oder noch nicht vollumfänglich von der Organisationsforschung untersucht wurden, so auch die sog. Creative Industries. Gegenüber einer Zugangsweise, die einem empirischen Feld mit A-priori-Wissen über Kreativität in der Mode oder die Fertigung von Modekollektionen begegnet, befürwortet die qualitative Methodologie das Folgen der Ereignisse in situ

12 | Swedberg definiert Theoretisierung wie folgt: "Theorizing includes what I have called observation as well as the activities [...] such as naming, conceptualizing, constructing typologies, providing an explanation, and so on. [...] Intuition, imagination, and abduction are also all indispensable to successful theorizing" (2012: 15). 
und währenddem sie entstehen (Czarniawska 2007a und 20I4a). Durch Feldforschung wird Gewöhnliches ungewöhnlich, denn sie kreiert eine spezifische Distanz zwischen Forschenden und deren 〉Objekten<. Einige Wissenschaftler argumentieren, dass diese Qualität besonders geeignet ist, um Organisationsforschung zu betreiben (Eberle/Maeder 20I6). Die ständige Herausforderung der Modeunternehmen, Neuerungen hervorzubringen, lässt die Möglichkeit des geplanten Managements von Modekreationen vermuten, was wiederum die Frage evoziert, wie das denn möglich ist. Deshalb lautet meine Fragestellung: Wie wird Kreativität, das heißt Neuerungen im Feld der Mode, entwickelt und designt, also hervorgebracht? Zwei Bedingungen sind dabei ausschlaggebend: Erstens muss vermutet werden, dass die Organisationsmitglieder eine Vorstellung davon haben, was eine Neuerung darstellt, und zweitens, dass die Organisationsmitglieder und das Management versuchen, die Neuerung modisch zu gestalten. Um die Dynamiken des Entwicklungsprozesses und Bedingungen der Produktion aufzudecken und zu beschreiben, bietet sich eine mehrbetriebliche Feldstudie als passende Strategie an (Flyvbjerg 2006).

\subsection{Standorte}

Zum Zwecke dieser Studie wurden zwei Modeunternehmen ausgewählt, anhand derer zwei Varianten von Modedesign und deren Produktionsformen illustriert werden. Flyvbjerg weist darauf hin, dass eine kleine Nummer an Untersuchungsschauplätzen dabei helfen kann, sich auf wenige anstelle von einer »Masse des Gleichen« zu konzentrieren und so von »der Stärke es Exempels« zu profitieren (ebd.: 228). Die Unternehmensgrenzen wurden aufgrund zeitlicher, geografischer und organisationaler Erwägungen festgelegt; die ethnografischen Feldphasen fanden in den Hauptsitzen der zwei Unternehmen statt, die beide in der Schweiz liegen. Die Unternehmen wurden zwischen Herbst 20I4 und Herbst 2017 auf häufiger Basis, jedoch unregelmäßig besucht. Obwohl der saisonale Rhythmus einen beständigen Zeitplan bezüglich der Entwicklung und Produktion einer Kollektion bietet, beschränkt sich diese Studie nicht auf den Umfang einer Kollektionseinheit, sondern nimmt die Rekursivitäten zum Ziel, die sich über längeren Zeitverlauf zeigen und musterhaft wiederkehren.

Des Zugangs und der Machbarkeit willen wurden Betriebe kleiner oder mittlerer Größe gewählt. Die Studie beschränkt sich auf die Hauptsitze in der Schweiz, denn mit der Globalisierung wurde die ehemals hiesige Textilindustrie spätestens in den I970er-Jahren ins Ausland verlegt. ${ }^{13}$ Zusätzlich wurde der

13 | Einige Spuren dieser Zeit lassen sich noch finden, so z. B. im Textilmuseum in St. Gallen, was dem Fall der Mode- und Textilindustrie der Schweiz historische Reichweite verleiht. 
Grad der allgemeinen Bekanntheit als weiteres Selektionskriterium festgelegt (vorzugsweise mittlere Berühmtheit und Präsenz, denn neue Unternehmen bestehen zumeist aus einer geringen Anzahl von Angestellten, demgegenüber kennzeichnen ältere Firmen höhere Eintrittsbarrieren). Die Firmen unterscheiden sich hinsichtlich Technologie, Artikelgruppen und Sortiment, Geschäftsstandorten, Formalstruktur (Abteilungen, Verträge, Geschäftsmodell) wie auch bezüglich ihrer Zielgruppen resp. Konsumenten (s. Tab. I). Eines der Unternehmen, I993 von der Designerin gegründet, ist auf Strickwaren (Männer-, Frauenkleidung) spezialisiert, die mittels spezifischer Technik und Strickmaschine gefertigt werden. Die andere Firma umfasst ein breites Sortiment: von sensorischen Produkten (Honig, Parfüm und Duftkerzen), bis hin zu Haushaltsartikeln (Tischsets, Servietten und Keramikware), Einrichtungsgegenständen (aus Kupfer gefertigte Tür- oder Kommodengriffe, Kissen, d. h. Bezüge und Futter) und Möbeln sowie Modeartikeln (Schmuck, Schuhe) und der Kleiderkollektion, die schließlich den Interessensfokus für das vorliegende Projekt abbildet. Offensichtlich erfordert diese Diversität eine generalisierende Marketingstrategie. Ursprünglich im Jahre I894 von Seidenhändlern gegründet, wurde die Firma I974 von der heutigen Besitzerfamilie übernommen und hat sich so von einem Textilhandel zu einem breit gefächerten Familienunternehmen entwickelt.

Firma I weist eine Werkstattfertigungstechnik des Prêt-à-porter aus, die auf einer handwerklichen, nicht aber individuellen Fertigung (wie das bei der Haute Couture der Fall war) gründet (Ziege 20II: I53 ff.). 
Tab. 1: Statistische Informationen und Verschiedenheiten

\begin{tabular}{|c|c|c|}
\hline $\begin{array}{l}\text { Statistische } \\
\text { Informationen / } \\
\text { Verschiedenheiten }\end{array}$ & Firma I & Firma 2 \\
\hline Gründungsjahr & I894 & I993 \\
\hline Mitarbeiterzahl* & $\begin{array}{l}30 \text { (I400 Stellen- } \\
\text { prozente) }\end{array}$ & $\begin{array}{l}\text { Io (6oo Stellenpro- } \\
\text { zente) }\end{array}$ \\
\hline $\begin{array}{l}\text { Ausbildung } \\
\text { Creative Director }\end{array}$ & $\begin{array}{l}\text { Familienmitglied, } \\
\text { keine formale Ausbil- } \\
\text { dung in Modedesign }\end{array}$ & $\begin{array}{l}\text { Formale Ausbildung in } \\
\text { Modedesign }\end{array}$ \\
\hline $\begin{array}{l}\text { Zusammenstellung } \\
\text { Designteam und } \\
\text { ständige Mitarbeitende }\end{array}$ & $\begin{array}{l}\text { Design, Produktion, } \\
\text { Schnitt/Designassis- } \\
\text { tenz, PR, Art Director, } \\
\text { Geschäftsleitung, } \\
\text { Personal/Verkauf }\end{array}$ & $\begin{array}{l}\text { Design, } \\
\text { Geschäftsleitung, } \\
\text { Produkt, } \\
\text { Strickmaschine, } \\
\text { IT/Programmierung }\end{array}$ \\
\hline Technologie & Partnering & Complete Stricktechnik \\
\hline $\begin{array}{l}\text { Sortiment } \\
\text { (antizyklische } \\
\text { Produktionsmethode) }\end{array}$ & $\begin{array}{l}\text { Breit gefächert, } \\
\text { umfangreich: } \\
\text { Seiden- und Wollschals } \\
\text { u. Foulards, Frauen- } \\
\text { kleider, Keramik, Ein- } \\
\text { richtungsgegenstände, } \\
\text { Schmuck, Parfüm, } \\
\text { Tee, Schuhe, Möbel, } \\
\text { Kinderspielzeug und } \\
\text { Accessoires }\end{array}$ & $\begin{array}{l}\text { Konzentriert, } \\
\text { differenziert: } \\
\text { Strickware für Damen } \\
\text { und Herren }\end{array}$ \\
\hline Marktausrichtung & Generalist & Spezialist \\
\hline Preissegment & Oberes Preissegment & Mittleres Preissegment \\
\hline
\end{tabular}

* Stand: 2017

So wird der Werkstattcharakter, insbesondere das Kunsthandwerk einer Neuerung, auf den Kleideretiketten detailliert beschrieben, indem die Beziehung zum Herstellungsort und den »artisans« erläutert wird. Demgegenüber basiert Firma 2 auf der technischen Basis der maschinellen Fertigung, die nach Fließprinzip produziert. Anhand der maschinellen Fertigungstechnik wird wiederholt die Möglichkeit erklärt, $\mathrm{zu}$ »bezahlbaren Preisen« in der Schweiz produzieren zu lassen - und das mit nur nachhaltigen Rohstoffen. Diese Produktionsverfahren scheinen viel mehr als Rechtfertigungsstrategie für Ladenpreise zu gelten als die Kreativität. 


\subsection{TeCHNIKEN}

Experteninterviews, Shadowing, teilnehmende Beobachtung und organisationale Dokumente als Instrumente der Selbstdarstellung beleuchten das Phänomen aus unterschiedlichen Winkeln, ohne Absicht, das ganze Bild darstellen zu wollen (Silverman 20I4: 47). Das rekursive Kombinieren der Forschungstechniken erlaubt es, das Phänomen zu studieren und mit den Ereignissen in Berührung zu kommen, wenn sie stattfinden (Flick 20II: 53).

Aufgrund dessen wurden zu Beginn der Studie semistrukturierte Experteninterviews durchgeführt. Diese wichtige Informationsquelle wurde mit anderen Techniken komplettiert, sodass umfangreiches Wissen über das soziale Phänomen generiert werden konnte (Flick 20II: 52 ff. Silverman 20I7). In diesem Fall wurden die aus den Experteninterviews generierten Informationen mit Konversationen aus den Beobachtungssequenzen und wenn möglich Shadowing von Personen und Objekten komplettiert (Czarniawska 2007a und 20I4a: 43 ff.).

Zusammengefasst fand die Feldphase über den Verlauf von drei Jahren in den Betrieben statt, wobei die zwei Projektteams während mehr als 230 Stunden beobachtet wurden. Insgesamt wurden ig Personen (die Interviews dauerten zwischen I5 Minuten und zwei Stunden) im Rahmen der Vorbereitungsund Experteninterviews interviewt, jedoch wurden nicht alle Angestellten der zwei Firmen interviewt oder begleitet. Die folgende Tabelle gibt eine Übersicht zu den Methoden und dem Feldmaterial; Interviews, Shadowing und teilnehmende Beobachtungssequenzen sowie organisational generierte Dokumente. 
Tab. 2: Liste des empirischen Feldmaterials

\begin{tabular}{|c|c|c|}
\hline Feldmaterial & Firma I & Firma 2 \\
\hline Interviews & $\begin{array}{l}\text { - } \text { Total: } 5 \\
\text { - } \quad \text { Design/Creative } \\
\text { - } \text { Director }(2 \mathrm{x}) ; \\
\text { - } \text { Art Director/CEO; } \\
\text { - } \quad \text { Produktionszuständige } \\
\text { - } \quad \text { PR-Experte; } \\
\text { - HR/Ladenchefin }\end{array}$ & $\begin{array}{l}\text { Total: } 4 \\
\text { - Geschäftsleitung; } \\
\text { - } \quad \text { Design/Creative } \\
\text { Director; } \\
\text { - Webmanager \& } \\
\text { Produktverantwortliche, } \\
\text { Designassistenz; } \\
\text { - Strickmaschinen- } \\
\text { operateur/ } \\
\text { Programmierer }\end{array}$ \\
\hline $\begin{array}{l}\text { Shadowing/ } \\
\text { teilnehmende } \\
\text { Beobachtungs- } \\
\text { sequenzen }\end{array}$ & $\begin{array}{l}\text { - } \quad \text { Modenschau (02.20I5), } \\
\text { - } \quad \text { AWrstellungssitzung } \\
\text { - } \quad \text { Erste Musterung } \\
\text { (Io.20I5), } \\
\text { - Zweite Musterung } \\
\text { (II.20I5), } \\
\text { - Praktikum, } \\
\text { vier Wochen } \\
\text { (oI./o2.20I6); } \\
\text { - Fitting vor Modenschau } \\
\text { (o2.20I6); } \\
\text { - Vorstellungssitzung } \\
\text { SSI7; Designer bei Cou- } \\
\text { turière (o9.20I6); } \\
\text { Fotoshooting (03.20I7) }\end{array}$ & 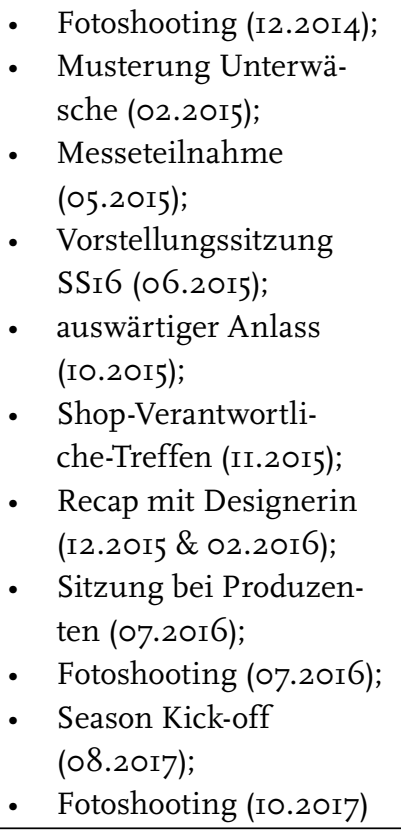 \\
\hline $\begin{array}{l}\text { Zusätzliche } \\
\text { Dokumente }\end{array}$ & $\begin{array}{l}\text { Kleideretiketten, } \\
\text { Newsletter, Kollektions- } \\
\text { broschüre, Flyer, Korres- } \\
\text { pondenz mit Produzenten }\end{array}$ & $\begin{array}{l}\text { Newsletter, } \\
\text { Kollektionsbroschüre, } \\
\text { Kleideretiketten, Werbung, } \\
\text { Skizzen (inkl. technischer } \\
\text { Beschreibungen) }\end{array}$ \\
\hline
\end{tabular}

Die Diversität der Informationsquellen fundiert die Glaubwürdigkeit des Feldmaterials und macht eine >polyphone< Beschreibung möglich, »since the perspectives of the people under study differ from one another« (Meyer 20I3: 3I). 
Die Verschiedenheit der Darstellungen eines Ereignisses wird mittels verschiedener Informationsquellen umfänglicher abgebildet. Im Grunde gibt es keine korrekte Darstellung oder Erzählung der Ereignisse: »Each telling, like light hitting a crystal, reflects a different perspective on this incident« (Denzin/Lincoln 20II: 8).

\subsubsection{Vorbereitungsinterviews}

In einer ersten explorativen Feldphase wurden Telefoninterviews mit Ausbildenden in Mode- oder Textildesign geführt. Insgesamt wurden neun Interviews geführt, die ungefähr I5 bis 20 Minuten dauerten. Während dieser Interviews fiel auf, dass es problematisch ist, den Begriff »Kreativität« anzusprechen. Die Informanten antworteten, dass Kreativität gelehrt werden kann; dass Kreativität schlicht individuelles Problemlösen sei; dass Kreativität sich von physischer Arbeit unterscheide; dass Kreativität die Freiheit der Gedanken und des Handelns meine; dass Kreativität visuell sein soll; dass Kreativität nicht routiniert, neu und unbekannt sei; dass Kreativität den Transfer von Erfahrung zu Form und Bild bedeute; dass Kreativität meine, mit alten Mustern zu brechen; oder dass Kreativität die Entwicklung zielgruppengerichteter Produkte hervorbringe - und so weiter und so fort. Mit diesen vorbereitenden Einschätzungen wurde offensichtlich, dass es an einem geteilten Kreativitätsbegriff fehlt, wie auch daran, wer kreativ ist oder was »kreativ« im Feld bedeutet. Als ich dann Zugang in mein >richtiges ₹ Feld erhielt, war ich bezüglich der Begriffsverwendung von Kreativität bzw. kreativ vorgewarnt.

\subsubsection{Standortinterviews}

In den Betriebsinterviews konnte mit den Angestellten der Betriebe Kontakt aufgenommen werden. Damit wurde die Absicht verfolgt, eine loyale und vertrauenswürdige Interaktionsbasis zwischen den Leuten im Feld und mir zu schaffen. McRobbie betont den Stellenwert des Jargons: »[... one] needs to be adroit with the kind of vocabulary typically used within specific fields of creative activity in order to have any sort of rapport with his or her respondents« (McRobbie 20I6: 942). McRobbie spricht sich deshalb für ausgeprägte Vorkenntnisse des Untersuchungsfelds aus. Trotz meines Interesses für ihre Arbeit musste ich davon ausgehen, dass »these people are less interested in precisely what you want to do than what sort of person you are« (Brown u. a. I967: 93, zit. n. Czarniawska 20I4a: 77).

Ich begann die Interviewserie mit der Person, welche die Rolle des Creative Directors/Directrice innehatte; danach wurden Interviews mit dem ganzen Design- und Entwicklungsteam sowie Personen mit unterschiedlichen betrieblichen Rollen geführt. Die Organisationsmitglieder sind »carrier[s] of knowled- 
ge« (Liebold/Trinczek 2009: 37), deren Entscheidungsfindung und Expertise die Kollektionsherstellung bedingen. Ihre Rollen waren folgende: Designer (oft als »Art Director« und »Creative Director/Directrice« bezeichnet), Angestellte aus dem Einzelhandel und der Produktion (Produktionsexpertin oder Produktund Webmanagerin), Techniker (IT und Maschinenoperateure) und administratives Personal (CEO, HR) sowie interne Marketing- und PR-Experten. Um eine vertrautere Atmosphäre zu schaffen, wurden die Interviews im snatürlichen Arbeitsumfeld der Interviewten (Büro, Sitzungszimmer, Fertigungshalle usw.) durchgeführt. Das Resultat waren Konversationen, die mehr informellen Gesprächen denn formalen Interviews glichen. Trotzdem wurden im Sinne eines semistrukturierten Interviews konkrete Fragen gestellt (Liebold/ Trinczek 2009). Alle Interviews wurden aufgenommen und transkribiert.

Das Ziel dieser im Betrieb stattfindenden Interviews war es, Verständnis zu gewinnen, was mit >Modemachen $<$ gemeint ist, wie die Organisationsmitglieder auf die Arbeit anderer Unternehmen reagieren und verweisen und diese in Beziehung mit der eigenen Arbeit stellen. Ich bin mir jedoch bewusst, dass einige der gestellten Fragen nicht nur praktisches Alltagswissen hervorrufen, sondern >Theorien< evozieren, die nur zum Zweck dieses Interviews von den Probanden entworfen werden. Zudem zeichnet die Kreativen eine bestimmte Selbstreflexion und Übung im Interviewgeben und Präsentieren aus, wobei nicht auszuschließen ist, dass diese Erfahrung auf das steigende Interesse der Gesellschaft an den Creative Industries und den Kreativen rückführbar ist (McRobbie 20ı6: 945). So ist das Gesagte nie vollständig, sondern eine Version gefilterter, interpretierter, reflektierter und in Worte gefasster Wahrnehmung der sozialen Wirklichkeit und stimuliert durch Fragen, die wenig mit dem Organisationsalltag zu tun haben. Genauso handelt es sich bei den Fragen um eine gefilterte Deutungsweise der Forscherin (Kruse 20I5: 60 ff.). Folglich machen Interviews nicht das gleiche Wissen zugänglich, wie über das Folgen bei der täglichen Arbeit erfahren werden kann.

Vor diesem Hintergrund muss gefragt werden, ob und auf welche Weise Kreativität in der Modebranche überhaupt beobachtet werden kann? Mit dem Beginn der Interviewreihe und der Definition des erklärten Ziels dieser Studie, nämlich die Rolle der Kreativität im Modedesign zu verstehen, offenbarte sich, dass es problematisch war (und ist), Kreativität als Zentrum der Arbeit in Modefirmen festzulegen und diese zum Ausgangspunkt zu nehmen. Die Gesprächspartner behaupteten oft, nicht kreativ oder überhaupt nicht in den Kreativprozess involviert zu sein. Folglich zeigt sich diese Herangehensweise aufgrund zweier Anlässe nicht hilfreich: Erstens verstanden die Gesprächspartner nicht genau, was sie gefragt wurden; zweitens ist »kreativ« ein Label, und jemanden oder etwas als kreativ zu bezeichnen, ist ein Prozess konstruk- 
tiver Praxis ${ }^{14}$ und deshalb vor allem von Interesse für die Organisations- oder Institutionenforschung, weniger aber für die Organisationsmitglieder. Der Prozess der Kreativitätszuschreibung verfährt implizit und passiv. ${ }^{15}$ Die Organisationsmitglieder brauchten den Begriff nicht in ihrem Alltag, und ihn einzuführen verlangt besondere Auseinandersetzung und Selbstreflexion, was nicht meiner Absicht entsprach. Eine Reaktion auf die Vorstellung des Forschungsvorhabens, die Rolle der Kreativität in Modefirmen zu studieren, lautete wie folgt:

Allerdings überschätzen Sie den Umfang des kreativen Backoffice von uns. Die Designerin, welche das Unternehmen gegründet hat, ist für das Design zuständig. In Zusammenarbeit mit der Geschäftsleitung wird der Kollektionsumfang und die Anzahl der neu zu gestaltenden Teile definiert. Sie gestaltet die Entwürfe und legt die neuen Kollektionsfarben fest. Bei Strickwaren obliegt es den Technikern des Produzenten, die Styles für die Strickmaschinen zu programmieren. Die Teile aus Trikotstoff werden in Zusammenarbeit mit einer Fertigungsdirectrice umgesetzt. Insgesamt handelt es sich pro Saison um nicht sehr viele Teile, da viele Modelle mehrere Saisons im Programm sind oder sogar als NOS [Never Out of Stock] Programm geführt werden. (Persönliche Korrespondenz, 16.01.2015)

Offensichtlich stellte ich die falschen Fragen bzw. musste feststellen, dass mein theoretisches Konstrukt des Kreativitätskonzepts nicht mit dem Alltagsverständnis der Akteure im Feld übereinstimmte (Sohlberg/Leiulfsrud 20I7). Ich musste den Fokus ändern - zumindest dessen Formulierung -, wenn ich von diesen Leuten akzeptiert werden wollte, und mich mehr in den Gegenstand, der mich interessierte, hineindenken, von dem ich noch immer glaubte, dass es sich um Kreativität handelt. Scheinbar verstand das Feld etwas Anderes unter Kreativität; und das, was ich meinte, war viel profaner, jedoch nicht weniger relevant. Diese Fragen, die sich auf Begriffe aus dem akademischen Diskurs (wozu Kreativität gehört) bezogen und - wie sich herausstellte - nicht zum täglichen Vokabular des Modesystems gehören, und die daraus resultierenden Schwierigkeiten veranlassten mich dazu, doppelt darüber zu reflektieren. Im besten Fall geben Interviews erste Hinweise dazu, was relevant und was irrelevant ist; Kreativität schien Letzteres zu sein. Von da an achtete ich

14 | Suchman (1995) argumentiert in ähnlicher Weise bezüglich der Art, wie Legitimität gewährt und instand gehalten wird. Weitere Beispiele dazu sind Bezeichnungen wie Reputation, Kunst oder Wissenschaft.

15 | Ein Beispiel eines aktiven Zuschreibungsprozesses ist aus der Musikindustrie bekannt. Die Billboard Music Charts stellen durch die "chart position" eine Rangordnung unter den gespielten Liedern und Alben her und fungieren als Bewertungs- und Feldstrukturierungsinstanz (Anand/Peterson 2000). 
deshalb viel mehr auf die Begrifflichkeiten und Konzepte, derer sich die Gesprächspartner bedienten. Modedesign ist deshalb ein besonders spannender Fall und erlaubt es, Kreativität da zu studieren, wo sie am meisten erwartet und am wenigsten von den Akteuren wahrgenommen wird.

Nachdem die Standortinterviews geführt worden waren, musste der Feldzugang weiter aufrechterhalten werden. Jeder Besuch barg seine eigenen Tücken des Feldzugangs und der Pflege dieses. Ich musste das »Recht des Zugangs« stets pflegen. ${ }^{16}$

\subsubsection{Shadowing}

Um sowohl tägliche Handlungen als auch Abweichungen der Organisationsmitglieder festzuhalten, beschloss ich zusätzlich, >Shadowing $\mathrm{zu}$ betreiben, das heißt, Personen und Objekten zu folgen, um die Bedingungen und zentralen Fragen der Entwicklung und Produktion neuer Produkte (in meinem Fall die saisonalen Neuerungen von Modekollektionen) zu verstehen. Diese Technik ist besonders einschlägig für organisationale Ethnografien: »Modern organizing, [...], takes place in a net of fragmented, multiple contexts, through multitudes of kaleidoscopic movements. Organizing happens in many places at once, and organizers move around quickly and frequently« (Czarniawska 2007a: I6). Weil Shadowing die Dynamik, Mobilität und Bewegungen der Personen und Objekte herausstreicht, ist es eine adäquate Technik für das facettenreiche - >kaleidoskopische< - Studium der Kreativität in Modefirmen. Hinsichtlich der Objekte muss bedacht werden, dass von der Entwicklung bis hin zur Produktion das im Laden ausgestellte, finale Produkt durch langwierige und iterative Herstellungsverfahren entsteht, weshalb das Resultat eine Möglichkeit unter vielen ist, da eine ganze Reihe anderer Optionen verworfen wurden. Einen >kaleidoskopischen< Blick auf die Ereignisse zu fördern heißt in diesem Fall, sich nicht nur auf das Endresultat zu beschränken, sondern auch die verworfenen Ideen und Entscheidungen als Teil des Prozesses zu erfassen. Diese Darstellungen geben mehr der kulturellen Werte und kognitiven Vorstellungen der Organisation preis als die nachträglich strategisch aufbereiteten Idealisierungen. Wenn immer möglich wurde im Modus von »closeup detailed observations « gearbeitet, in der Hoffnung, eine Beschreibung des »organizational life as it unfolds in its natural settings with intimate first-hand

16 | McRobbie spricht die Schwierigkeit der direkten Beobachtung der Designer bei der Arbeit an, wobei der Zugang für Beobachtende aus der Wissenschaft besonders umständlich gestaltet wird (McRobbie 2016: 938). Demgegenüber stehen Dokumentarfilme wie beispielsweise "Dior et moi« (Regie: Frédéric Tcheng [2013]), in dem die tägliche Arbeit des Modeschaffenden Raf Simons porträtiert und begleitet wird und die als Idealvorstellung ethnografischer Forschung fungieren. 
observations from a particular subject's frame of reference « zu leisten (Van de Ven/Poole 2005).

Durch das Shadowing und Teilnehmen versuchte ich, mich so zu platzieren, dass sich die Gesprächspartner wohlfühlten. Als mir dann von der HR-Managerin offerierte wurde, eine Bluse und einen Schal aus der aktuellen Kollektion auszusuchen, nahm ich dies als Beweis der Anerkennung, dass sie mich als >eine von ihnen< wahrnahmen und deshalb wollten, dass ich mich in ihrer Uniform kleide. Es schmeichelte mir, denn diese >optische Angleichung < half mir, nicht zu viel Aufmerksamkeit auf die Forschungsaktivität zu lenken (Czarniawska 20I4: 54) und camouflierte meinen Status als >Fremdling gegenüber deren Kundschaft. Später wurde diese Deutungsweise bestätigt - und durchaus positiv wahrgenommen -, denn wie eine Mitarbeiterin kommentierte, wurde ich mehr und mehr zum Teil des Unternehmens. Es war ein Zeichen ihres Vertrauens und gewährte mir Zugang zu unterschiedlichen Arbeitsplätzen. Zusätzlich förderten meine Feldaufenthalte das Verständnis der Organisationsmitglieder für meine Forschungsarbeit. Besuche, die höchste Einbindung verlangten (eingeladen für teilnehmende Beobachtungen von den Beobachteten selber, aktive Interaktionen), wechselten sich mit Besuchen $\mathrm{ab}$, in welchen ich an der Peripherie der Handlungen blieb. Dadurch konnte ich trotz des Angleichens eine Außenseiterrolle (outsidedness) aufbauen (ebd.). Eine andere Technik, die meinen Außenseiterstatus signalisierte, war das offensichtliche Verfassen der Feldnotizen. Das Anfertigen von Mitschriften wird als eines der zentralen Werkzeuge der Feldforschung angesehen; daher ist es Teil der täglichen Beobachtungssequenzen (Emerson/Fretz/Shaw 20II). Das offene Verfassen solcher Mitschriften (das in Kontrast mit dem Verfassen solcher außerhalb oder nach der eigentlichen Beobachtung steht) unterbindet jegliche Art von Misstrauenspolitik und fördert die Neugierde am Forschungsprojekt (Lofland I97I: III).

Die während des Tages hergestellten Feldnotizen wurden zu Tagesende in vollständige, schematische Berichte umformuliert (Emerson/Fretz/Shaw 20II). Die Kopfzeile des Formulars folgt Richards Instruktionen zum »deskriptiven Codieren« (20I5: Io6 ff.), das Informationen zum Dokument, dem Unternehmen, den Akteuren und deren Positionen wie auch der Örtlichkeit und weniger zu Prozessen und Textpassagen enthält.

Dieses schematische Formular für Feldnotizen beinhaltete zudem ex ante Fragen und Erwartungen an die kommende Beobachtung sowie Raum für Ex-post-Zusammenfassungen und Fragen, die in weiteren Beobachtungen bedacht werden sollten. Die Beobachtungssequenzen wurden in Intervalle aufgeteilt, die je eine Handlung umfassten; neue Handlungen bedeuteten, dass sie in einem neuen Notizfeld beschrieben wurden. Zum Beispiel fungierte während des Fotoshootings eine Pose eines Models als ein solches Intervall. Wenn das Model sich umkleidete und dafür das Set verließ, zählte das als 
anderes Intervall, wie auch dann, wenn der Fotograf und die Designerin die fotografierten Bilder durchschauten. Schießlich kam das Model zurück aufs Set, um mit einem neuen Outfit fortzusetzen, dann wurde ein neues Intervall angebrochen und Notizen wurden in einem neuen Feld festgehalten. Die rechte Spalte war reserviert für Kommentare. Aus diesen Kommentaren wurden »Themencodes« entwickelt, die sich meist auf ein Intervall beschränkten und üblicherweise erste Interpretationen beinhalteten (Richards 20I5: IIO ff.). Am Schluss des Formulars befindet sich ein Feld, in dem das während der Beobachtungssequenz gesammelte Feldmaterial aufgelistet wurde. Dadurch wurde das Feldmaterial systematisiert (Silverman 20I4: 83 ff.). Diese Berichte, die Interpretationen und die Codes wurden wiederholt in einem regelmäßig stattfindenden Analysetandem besprochen.

Feldbeobachtungen wurden mit kurzen Interviewsequenzen kombiniert, in denen je nach Bedarf nach Erläuterungen gefragt wurde. Gleichermaßen ergaben sich hie und da Folgeanfragen, in denen nach verworfenen Optionen und Entscheidungen gefragt wurde, wobei sich auch in diesen Prozessen gezeigt hat, dass es etablierte Verfahrensweisen gibt, die allerdings an die jeweilige Situation angepasst werden. Letztendlich konnten die beobachteten Aktivitäten (Skizzieren, Produktionssitzungen, technische Musterzeichnungen oder Ausführungen für Schneider) und Ereignisse (neue Modelle, angepasste Modelle, Familientraditionen, technologische Ausrichtungen) in einem kontinuierlichen Strahl vergangener Entscheidungen und künftiger Pläne verortet werden, welche »intersubjektive Momente des sozialen Handelns und Erlebens« kennzeichnen (Kuhlmann 2005: 80).

Wird das Praxisfeld von außen beobachtet, so werden Optionen freigelegt, welcher sich die Organisationsmitglieder nicht bewusst sind oder welcher sie sich nur »in the moment of reflection, of observing, of not acting « (Czarniawska 20I4a: 5) bewusst werden. Aus diesem Grund geht es bei der Technik des Beobachtens und Shadowings nicht darum, etwas besser zu wissen, sondern etwas anders zu sehen - durch das Kaleidoskop (Czarniawska 2007a: I6) oder das Kristall (Denzin/Lincoln 20II: 8). Diese Differenzen werden gemeinhin erwartet und zu wertvollen »Wissensquellen « (Czarniawska 20I4a: 45). Mittels Beobachtungs- und Shadowing-Sequenzen konnte das Wissen über Entwicklungs- und Produktionsprozesse sowie Entscheidungsfindung und organisationale Verwaltung erweitert werden.

\subsubsection{Dokumente und Selbstdarstellung}

Zusätzlich zu den Interviews und den Feldnotizen kamen ungefähr 250 Seiten Dokumente organisationaler Selbstdarstellung. Organisationen kontrollieren die Selbstdarstellung so, dass sie dem Vorteil der Unterstützung durch zentrale Kundenkreise und deren Zustimmung nützt (Elsbach/Sutton I992). 
Dokumente werden hierbei als »Materialisierung von Kommunikation« verstanden (Froschauer 2009: 326). Die Materialisierung ist Ausdruck - ein finalisiertes Ergebnis, bereit zur Verbreitung - der sozialen Organisation, Konstruktion und Produktion von Kreativität. ${ }^{17}$ Die Darstellungen schildern die erwünschte Fremdwahrnehmung. Newsletter, Preisschilder und Etiketten, Ausstellungskataloge, Kollektionsbroschüren und Flyer, Medienberichte, z. B. Berichte aus unterschiedlichen Branchenzeitungen oder Magazinen, enthüllen die kollektive Konstruktion des organisationalen Porträts (inklusive Kreativität), also die organisationale Selbstdarstellung. Ein Vorteil solchen Materials und dessen Analyse ist, dass es Zeit und Raum überdauert und fern von aktuellen Forschungsaktivitäten besteht (Ventresca/Mohr 2005).

To summarize, the significance of narrative data lies not just in their richness and near universal availability, but in the fact that they are the same kind of data that organizational members use to plan, enact, interpret, and evaluate their own actions and those of others" (Pentland 1999: 717, Hervorh. im Orig.).

Schließlich wird der Vorteil dieser Datengattung betont: Diese Daten verändern sich nicht und bestehen unabhängig von der Planung, Interpretation und Analyse.

\subsection{Analyse Oder was das Feldmaterial erzÄhLt}

Fallstudien sollten sich als kohärente Erzählung lesen: »Good case [and site] studies should be read as narratives in their entirety« (Flyvbjerg 2006: 24I Hervorh. der Verf.). Die vorliegende Betriebsstudie widmet sich dem Phänomen der Kreativität - im Spezifischen in der Modeindustrie und allgemein in den Creative Industries.

Meistens geschehen das Sammeln und die Analyse des Feldmaterials gleichzeitig, und selten können klare Grenzen zwischen den einzelnen Phasen gezogen werden. Während die Gesprächspartner der Entwicklung und Produktion und des Designs im Betrieb, an Messen, bei Fotoshootings oder Modenschauen weiterhin besucht wurden, wurde das gesammelte Material durchgesehen. Durch daraus gewonnene Einsichten und Erkenntnisse wurden die weiteren Situationen im Forschungsvorhaben geleitet und bestimmt. Dazu blieb ich stets in Kontakt mit den Organisationsmitgliedern, bat diese

17 | Methodisch ist es von Bedeutung, dass finalen Versionen organisationaler Dokumente eine Vielzahl von Selektionen vorausgehen, die in ihrer finalen Erscheinungsform keine weiteren Rückschlüsse auf die ursprünglichen Ideen respektive verworfenen Optionen zulassen. 
um aktualisierte Informationen zur Kollektion oder zum Betrieb. So konnten die Organisationen mich weniger leicht vergessen.

In einem ersten Analyseschritt wurden die Interviewtranskripte und die Beobachtungsprotokolle gelesen und rudimentär, offen kodiert, um die mit der Entwicklung und Produktion von Neuerungen in Beziehung stehenden Kategorien zu erkennen. In einem zweiten Schritt wurde dieses Vorgehen wiederholt. Die Analyse ist ein iterativer Prozess, und so wurde das Material mehrere Male durchgearbeitet, um eine generellere Zusammenstellung zu erhalten. Dies erforderte einen kontinuierlichen Dialog zwischen Feldmaterial und den bestehenden Theorien. Durch die wiederholte Überarbeitung der Relevanz und Überschneidungen konnten die einzelnen Kodes in einem intensiven, aber fruchtbringenden Verfahren entwickelt, d. h. entworfen, verändert, modifiziert, gelöscht, zusammengefasst, getrennt oder umsortiert und in Kategorien gefasst. Richards formuliert es wie folgt: »Rather than just store information or name the topic of the text, you are considering the meanings in context, and creating categories that express new ideas about the data, coding to gather and reflect on all the data related to them. This is qualitative research!« (20I5: II2). Die Kodes sind Mittel, um den Sinn des Textes zu verstehen und zu konzeptualisieren. Das Kodieren fand mithilfe der Software atlas.ti statt (kein automatisches Kodieren wurde angewendet).

Zum Zweck der intersubjektiven Nachvollziehbarkeit und Verständlichkeit der analytischen Konzepte, Kategorien und Themen fanden regelmäßig Analysetandemtreffen statt, die mindestens aus einer Person und mir bestanden und die dazu dienten, Probleme zu präsentieren und auszuhandeln. Muster in und zwischen den Betrieben, das heißt Gemeinsamkeiten und Verschiedenheiten (s. Tab. I), wurden aufgelistet, um so mit bestehenden, vereinfachenden Rahmen vermeintlich gleicher Modelle zu brechen. Das macht »a lot out of a little« (Silverman 20I3: I4I). Zum Beispiel stellen beide Unternehmen ähnliche Produkte her, jedoch auf zwei verschiedene Weisen: Der Spezialist konzentriert sich lediglich auf die auf einem 3-D-Strickcomputer hergestellte Strickware, während der Generalist auf erfahrenes und in klassischer Schneiderei und Textildruck ausgebildetes Personal zurückgreift. 


\section{Zur Feldstrukturierung der Creative Industries}

Wissenschaftliche Beiträge zur Organisation der Kulturproduktion stammen aus den I970er-Jahren (Hirsch 1972; Becker 1974; Kadushin 1976). Seit den späten I99oern befasst sich ebenfalls die Politik der Nationalstaaten mit dem Gegenstand Kultur- und Kreativitätsförderung (DCMS 200I; UNCTAD 2008a). Die damit verknüpften Geschäfte wurden mit dem Begriff »Creative Industries/Economy « betitelt und richteten sich an eine Vielzahl von Akteuren, darunter Nationalstaaten, Kulturverwaltung, Gewerbeverbände, Bildungsprogramme oder die Wissenschaft. Als im Jahr 20I4 dann offiziell der Begriff »creative turn « (Sriraman/Harris 20I4) vorgeschlagen wurde, war Kreativität als gesellschaftlicher Leitsatz schon weit vorgedrungen und omnipräsent, beispielsweise in wirtschaftspolitischen Belangen wie Wirtschaftsförderung und Stadtentwicklung oder als Gegenstand interdisziplinärer Forschung.

Das Resultat nationalstaatlicher Regulierungen, organisationalem Agendasetting und gesellschaftlicher Maxime war die Konfigurierung eines neuen, organisationalen Feldes, ein soziologisches Konstrukt, das sich auf »particular structures and relations and meaning systems to which an organization relates « bezieht (Scott 1994: 208). Typische Merkmale eines Feldes sind u. a. gegenseitige Wahrnehmung der Feldmitglieder, eine erhöhte Interaktionsfrequenz und gesteigerter Informationsfluss sowie gleiche Bedingungen für organisationale Aktivitäten (DiMaggio/Powell I983; Bourdieu 1993; Scott 1994; Fligstein/McAdam 20II; Wooten/Hoffman 2013). Auf diese Weise sind die Creative Industries das Produkt eines Beziehungs-, Informations- und Ressourcennetzwerks, das sich um eine zentrierte Organisationsform $-z$. B. ein Filmset oder einen Architekturwettbewerb - formiert (Powell/Smith-Doerr I994; Caves 2002 und 2012).

Ein solches (Sub-)Feld ist auch die Mode. Die Feldstruktur der Modebranche ist netzwerkartig, von unterschiedlichen Organisationsgrößen geprägt, und geografisch disparate Akteure stehen in unregelmäßigem Kontakt miteinander. Die Mode hat sich im letzten Jahrhundert zu einem institutionalisierten und internationalisierten Organisationsfeld entwickelt. Dabei fällt auf, 
dass vor allem die Produktion und der Konsum von (Neu-)Kreationen auf der Feldebene koordiniert werden, während die Entwicklung dieser in der Organisation stattfindet. ${ }^{\mathrm{I}}{ }^{8}$ Unter der Schirmherrschaft von Unternehmen werden in Projekten neue Ideen koordiniert, von sektoralen Produktionsnetzwerken zu kreativen Gütern produziert und im organisationalen Feld konsumiert. Die vertiefte Auseinandersetzung mit der Mode als Sektor der Creative Industries vermittelt Einsichten darüber, wie ökonomische Belange mit künstlerischem Ausdruck kombiniert werden, indem die Umwelt beobachtet und auf ihre Dynamiken reagiert wird und Veränderungen anschließend am operativen Betrieb ausgerichtet werden (Kap. 6.2). Das Feld der Mode geht aus den komplexen Wechselwirkungen »between the aesthetic and economic aspects of fashion practices engaged by actors constitutive of this field (fashion companies, retail consultancies, flagship luxury organizations, designer sand designers associations, advertising and publishing companies, and global communication directors) « hervor (Petkova 20I6: 57). Als netzwerkartige Gebilde sind Felder leichtfüßig unterwegs und können schnell auf die Dynamiken der institutionellen Umwelt reagieren, was insbesondere für die Zeithaftigkeit und veränderlichen Bedingungen in der Kreativitätshervorbringung und Kulturproduktion von Vorteil ist (Powell/ Smith-Doerr 1994: 38I).

Das folgende Kapitel beabsichtigt dreierlei: Erstens soll es eine Übersicht zum Begriff »Creative Industries« geben, der sich von wissenschaftlichen Konzeptionen zu kulturpolitischen Definitionen bewegt. Zweitens wird der Inhalt der Creative Industries anhand von empirischen Feldbeschreibungen erläutert, die weiter der Besprechung dienen, warum es sich bei den sogenannten Creative Industries um die Entstehung eines organisationalen Feldes (oder vieler Felder) handelt. Schließlich, drittens, mündet die empirische Diskussion in der konzeptuellen Darstellung einer bestimmten Creative Industry, nämlich der Strukturen und Merkmale des Modesektors.

18 | Die Mode stellt selber ein Feld bzw. ein Subfeld der Creative Industries dar. Von Feldern (Mode, Creative Industries) ist die Rede, wenn alle gesellschaftlichen und ökonomischen Akteure, die zur Produktion, Distribution und auch zum Konsum bei- 


\subsection{Zum Feldbegriff der Creative Industries}

Die Kulturproduktion ist ein soziologisches Phänomen, weil das kollektive Unternehmen der Produktion symbolisch-kultureller Güter und deren Wert alle daran beteiligten Akteure umfasst (Hirsch 1972; Becker 1974; Bourdieu I993). Bourdieu bezeichnet den Gegenstand kreativer Hervorbringung nicht als individuelles Problem, sondern als Aufgabe eines Kollektivs:

[...] the subject of artistic production and its product is not the artist but the whole set of agents who are involved in art, are interested in art, have an interest in art and the existence of art, who live on and for art, the producers of works regarded as artistic (great and small, famous - i. e. 'celebrated - or unknown), critics, collectors, go-betweeners, curators, art, historians, and so on. (Bourdieu 1993: 103)

Die Kulturproduktion und deren soziale Wertekonstruktion wird zum Inhalt eines organisationalen Feldes, nämlich den Creative Industries. Die Sektoren der Creative Industries unterscheiden sich von anderen Industrien, weil sie die Wertschöpfung auf Kreativität und Erneuerung beziehen (Amabile u. a. I996; Oldham/Cummings i996).

\subsubsection{Vom Industriesystem zum organisationalen Feld}

Die Ursprünge der Debatte um die Kulturindustrie entstammen der Frankfurter Schule der I930er- und I940er-Jahre. Die Diskussion um die Kulturindustrie erhält mit Paul M. Hirschs Aufsatz »Processing Fads and Fashions: An Organization-Set Analysis of Cultural Industry Systems« im Jahre 1972 neuen Aufwind, an die Becker mit dem Konzept der »Art Worlds « zwei Jahre später (I974) anschließt und von Kadushins Idee der »Networks and Circles in the Production of Culture« (I976) ergänzt wird. Hirsch definiert die Kulturindustrie als

cultural industry systems, comprised of all organizations engaged in the process of filtering new products and ideas as they flow from rcreative personnel in the technical subsystem to the managerial, institutional, and societal levels of organization. (Hirsch 1972: 642)

tragen, gemeint sind (ähnlich dem Konzept gesellschaftlicher Sektoren nach Scott/ Meyer 1991). Das Feld lässt sich in ökonomische Sektoren (betriebswirtschaftliche Branchen) einteilen. Ein Sektor umfasst alle Akteure, die zur Produktion und Distribution eines bestimmten Marktsegmentes beitragen. Ein Beispiel ist der Sportbekleidungsmarkt, ein anderes die Uhrenindustrie. Beispiel der hier vorliegenden Arbeit sind zwei unabhängige Modefirmen im mittleren Preissegment. 
Hirschs »industry system « wird in Bezug zu Konzepten wie »societal sector« (Scott/Meyer I99I), »relational space« oder dem organisationalen Feld gesetzt (DiMaggio/Powell I983; Wooten/Hoffman 2013). Dies sind Ansätze, die den Fokus weg von der einzelnen Organisation auf ihre Umwelt sowie die Einbettung und Vernetzung richten. Diese Aspekte sind auch für die Hervorbringung von Kreativität essenziell. Diese Ansätze verstehen Industrien als Konstellationen von Akteuren, die nicht nur relational verbunden sind und sich unabhängig von der geografischen Distanz oder Nähe berücksichtigen, sondern auch gleichen normativen Regeln folgen und unter gleichen Bedingungen tätig sind (Scott I994: 206). Gleichermaßen betont Hirsch die wechselseitigen Abhängigkeiten der Akteure im Industriesystem:

The concept of industry system takes the focus of attention away from any single firm or role in the sequence of discovering, producing, and delivering a product, redirecting attention to the interconnections and interdependencies between them in order to get to the final (finishedı) product or outcome. (Hirsch 2000; Hervorh. im Orig.)

So richten Organisationen ihre Aufmerksamkeit auf die Umwelt, wobei durch gegenseitiges Beobachten Subfelder, d. h. Sektoren wie z. B. der Musikmarkt oder das Feld der Presse, entstehen. Ein nennenswertes Beispiel der Entstehung eines organisationalen Feldes im Modebereich ist die Arbeit von Khaire zum indischen Modemarkt, in der das parallele Aufkommen der zunehmend global ausgerichteten Industrie sowie der Modebildungsinstitute, Magazine und spezialisierten Einzelhandelsgeschäfte herausgearbeitet wird (Khaire 20I4; Khaire/Hall 20I6). Das Feld der Creative Industries besteht demnach aus mehreren Sektoren oder Branchen. In der Kreativitätsliteratur sind ähnliche Diskussionen weiter unter den Begriffen »Art Worlds« (Becker 2008), »Circles in the Production of Culture (Kadushin I976), »Creative Class und City« (Florida 2006), »Regime des ästhetisch Neuen« (Reckwitz 20I4: 43 ff.) oder auch »Ecologies of Creativity« (Grabher 200I) zu finden. Zunehmend werden Stimmen aus Wirtschaft und Kultur laut, die auf die Zerstreuung und Unterschiedlichkeit der Creative Industries verweisen und eine Differenzierung in verschiedene (kreative) Sektoren mit je eigenen Ausprägungen als notwendig erachten (McRobbie 2016: 937).

DiMaggio und Powell definieren das organisationale Feld wie folgt: »By organizational field we mean those organizations that, in the aggregate, constitute a recognized area of institutional life: key suppliers, resource, and product consumers, regulatory agencies, and other organizations that produce similar services or products« (I983: 64 f.). Felder zeichnen sich durch interorganisationale Strukturen wie Hierarchie und Koalitionsmuster, gegenseitiges Bewusstsein und erhöhte Kommunikation aus. Weiter sind auch kognitive Komponenten wichtig: 
Organizational fields are made up of both cultural and behavioral elements. The former refers to the meaning systems and symbolic frameworks that define and give coherence to a set of behaviors, together with the constitutive rules that define the utilities and capabilities of actors and the normative rules that specify appropriate forms of conducts: the rules of the game. (Scott 1994: 207)

Anhand dieser Spielregeln wird ein geteiltes Bewusstsein konstruiert, d. h. Identitäten und Aufmerksamkeitsschemata, eine feldspezifische, zentrale Handlungslogik und geteilte, selbstverständliche Situationsdefinitionen und Annahmen bilden sich heraus (ebd.: 207). So können Feldmitglieder zwischen kreativen und nicht kreativen Akteuren unterscheiden, wissen über das eigene Kreativitätspotenzial und den Neuheitsgehalt des Designs Bescheid und kennen sowohl die Instanzen, die das Label verleihen können, als auch deren Beurteilungsmacht. Diese Spielregeln werden im Feld ausgehandelt und über Interaktion und Kommunikation gebildet und kontinuierlich aktualisiert (Czarniawska-Joerges 20II). Das geteilte Glaubenssystem ist nicht nur ein Feldzugehörigkeitsmerkmal der Mitglieder, sondern auch das zentrale Charakteristikum des Feldes.

Kadushins Konzept der »Zirkel« (I976) kommt kleineren, dichten Netzwerkkonfigurationen mit einer Kerngemeinschaft nahe, die aufgrund gemeinsamer Interessen Bedürfnisse gestalten, auf Zeit angelegt sind und so lange »unsichtbar« bleiben, bis sie sich in erhöhtem Informationsfluss oder in Verträgen zeigen. Eine Gemeinsamkeit der organisationalen Felder ist die unklare Grenzziehung. Sie werden erst durch die analytische Festlegung deutlich. Aufgrund der internen Spezialisierung sind Zirkel immer auch auf externe Zusatznutzen anderer Zirkel angewiesen. Ein Zirkel umfasst sowohl ehemalige wie aktuelle Mitglieder, die räumlich und zeitlich unabhängig voneinander auf ihre Werke verweisen. Die Diffusion des relevanten, organisationalen Wissens über Raum und Zeit ist ein Merkmal der Creative Industries. Kadushins Darstellungen verdeutlichen schließlich nicht nur die vernetzte, sondern auch die kooperative Struktur der Herstellung expressiver Güter.

So können zum Beispiel die Repräsentation, Koordination und Diffusion neuer Ideen als das Kerngeschäft der Creative Industries verstanden werden (Potts u. a. 2008: I76). Das Feld zeichnet eine Rationalität der Erneuerung aus, so das Argument, weshalb Neuheit der zentrale Nutzwert ist. ${ }^{19}$ Die zentralen

19 | Entsprechend ist die Unterscheidung von Kreativität und Innovation eine qualitative: während mit Innovationen der praktische Anspruch in Form eines technisch-utilitaristischen Fortschritts oder einer Verbesserung der Umstände verfolgt wird, zeichnen sich symbolisch-kreative Güter durch keine fundamentale Funktion aus, außer der, neu zu sein (Beckert 2011; Hutter 2011; Mears 2011; Stark 2011; Krämer 2014). Als Beispiele für Erneuerungen in ästhetisch-kreativen Industrien werden auch Be- 
wie auch peripheren Akteure des Feldes übernehmen das Organisieren symbolischer Werte, ökonomischer Belange und institutionellen Wissens (Jones/ Thornton 2005). Als Folge der unterschiedlichen Logiken und Identitäten befinden sich die Creative Industries und insbesondere der Modesektor in einem Spannungsverhältnis zwischen Kunst und Kommerz und sehen sich mit der Anforderung konfrontiert, kreativen und wirtschaftlichen Erwartungen zu entsprechen (Cappetta/Cillo/Ponti 2006; Moeran 2008; Titton 20I2; Aspers/ Godart 20I3; Vangkilde 20I3).

Jüngere Forschung der Creative Industries sieht die Entwicklung neuer Informations- und Kommunikationstechnologien und neue Möglichkeiten der Kultur- und Massenproduktion als weitere Ursache für deren Entstehung und Aufstreben:

The rise of cultural industries goes hand in hand with the emergence of new technologies such as printing, sound recording, photography, film, video, and the Internet. These new technologies give advantage to economies of scale in production, distribution, and marketing. As in other sectors, this leads to large corporate entities whose main business is to create, market, and distribute cultural goods. It also produces new occupations and new skills, and ecology of large and small firms that specialize in creating content and assisting delivery. (Lampel/Shamsie/Lant 2008b: 7)

Während ältere technologische Errungenschaften wie Fotografie und Film oder Musikaufzeichnungstechnologien zumeist einzelne Sektoren revolutionierten, betreffen das Internet und die Digitalisierung alle Creative Industries.

Die Begegnung mit Neuem ermöglicht im ersten Augenblick Momente des Experimentierens, weshalb der technologische Wandel auch für die (Weiter-)Entwicklung der Güter der Creative Industries fundamental war. Durch die systematische Nutzung neuer Technologien und digitaler Medien werden neue Marktnischen erschlossen, die sowohl die Produktion, den Vertrieb, die Vermarktung wie auch den Konsum neuer Güter beeinflussen (Lampel/ Shamsie/Lant 2008b: 7). Daraus entstehen neue Geschäftszweige, die sich auf Teilprozesse wie die Herstellung, die Distribution oder die Vermarktung solcher Produkte spezialisieren (z. B. visuelle Kommunikation oder Management und Booking von Musikbands), und somit entwickeln sich neue Berufsfelder und Fähigkeitsprofile.

Die von Hirsch vorgestellten Managementideen fanden auch in anderen Industrien Anklang, weil sie Schwierigkeiten bezüglich Planbarkeit, Resonanz aus der Umwelt und technologischem Wandel mit zielführenden Praktiken u. a. der Auslagerung zahlreicher Abteilungen und Arbeiten konzeptuell zu

nutzerfreundlichkeit oder "off use «-Design genannt (Krause-Jensen 2013; Moeran/ Christensen 2013; Vangkilde 2013). 
lösen wussten, und sie wurden dadurch zum interdisziplinären Exportschlager (Hirsch 2000: 357).

\subsubsection{Vom interdisziplinären Exportschlager zum ökonomischen Marktpotenzial}

Die Produktionslogik der Creative Industries schließt an die der Kulturwirtschaft an, denn beide produzieren per Modifikation, wobei Folgeprodukte an Vorgängerprodukte und Erfahrungen anschließen (Caves 20I2: 4). ${ }^{20}$ Ein Beispiel dafür ist die Studie von Djelic und Ainamo (2005), die wertgenerierende Strategien und die Logik der Erneuerung der Mode auf die Telekommunikationsindustrie anwenden.

Die Selbstbeschreibung der Industrie verzeichnet zunehmend ein wissenschaftliches Interesse. Durch die Verwissenschaftlichung wurde Kreativität zum erwünschten Label einer Industrie, der besonderes Potenzial zugeschrieben wurde. Erstens sollte der bis dahin unzugängliche und vermeintlich nutzlose Kulturmarkt nun als Creative Industries die ökonomischen Markttätigkeiten stärker mit lokalem Wissen und Darstellungsformen verbinden, zweitens sollten dadurch interessante und lukrative Expansionsmöglichkeiten entstehen, und drittens sollte damit der als notwendig erachtete ökonomische Spielraum zur Erarbeitung wirtschaftlicher Entwicklungsstrategien bereitgestellt werden (Hartley u. a. 20I3: 79). Damit verschob sich das Interesse der Creative-Industries-Forschung von dem »environment of the organizations to the organizations of the environment (Scott 2008: I6, Hervorh. der Verf.) Diese konzeptuelle Neuorientierung verstärkt die Feldwahrnehmung der Creative Industries. Diese Schnittstelle von Wirtschaft, Kultur und Innovation wirkt als optimales Forschungsterrain:

First, it is valuable because it mainstreams the economic value of culture, media and design. [...] Second, it brings together in a provisional convergence a range of sectors which have not typically been linked with each other. [...] Third, the sectors within Creative Industries [...] move from the resolutely non-commercial to the high-tech and commercial. This continuum moves from the culturally specific non-commercial to the globalised and commercial, where generically creative, rather than culturally specific, content drives advances. (Hartley u. a. 2013: 59)

In den Creative Industries konvergieren wirtschaftliche, technologische und politische Schwerpunktsetzungen zu interdisziplinären Debatten. Weiter rücken die Kultur, Medien und Design in den Fokus wirtschaftlicher Wertschöpfung. Zudem führt das Feld unterschiedlichste Sektoren zusammen und er-

20 | Aktuelle Darstellungen erfassen die Kulturwirtschaft als Teil der Creative Industries. 
streckt sich vom Gemeinnützigen bis zur Spitzentechnologie. Ein Feldbegriff, wie ihn die Institutionen- und Organisationsforschung kennt, ermöglicht die Beachtung unterschiedlicher disziplinärer Aspekte und Erwartungen. Weiter unterstützt der Feldbegriff das Verständnis und die Relationen zwischen der Makroebene (globale Themen) und der Mikroebene (lokale Interpretationen) (Wooten/Hoffman 20I3: I4I).

Die Schnittstellenposition und Überbrückungsleistung ruft Spannungen zwischen Akteuren und Prozessen hervor. In der Kreativitätsliteratur wird von einer integrativen Leistung dieser Spannungen und dem Effekt der Vereinbarung ambivalenter Tendenzen (Kunst - Kommerz, Industrie - Kultur, Neuerung - Wiederholung) gesprochen, welche sich schließlich zum konstituierenden Merkmal der Selbstbeschreibungsformel entwickeln (Barrett I998; Lampel/Lant/Shamsie 2000; Madjar/Oldham/Pratt 2002; Gilson u. a. 2005; Hargadon/Bechky 2006; DeFillippi/Grabher/Jones 2007).

Mit der Zeit hat die Wissenschaft ein Interesse für Kreativität fern typischer Industrien entwickelt und sich von der Kreativitätsdiskussion der Kulturproduktion und den Creative Industries abgewandt. Katherine Chen rät deshalb, »to consider creativity in a variety of organizations, rather than just those that are known for creative outputs or practices« (2012: 624). Kreativität in einer Vielzahl von Industrien zu untersuchen, trägt zur kritischen Auseinandersetzung mit dem Gegenstand bei und erweitert deren Anwendungsbereich. Diese kritische Betrachtung reflektiert zudem die Selbstbestätigung durch das Label, die vor allem in Rollenbezeichnungen, wie der des Creative Directors, oder in Branchen, wie jene der Creative Industries, unhinterfragt existiert. Gleichzeitig sollte aufgrund der Tatsache, dass das Label unhinterfragt weiterbesteht, die Frage gestellt werden, weshalb denn einige Industrien kreativ(er) sind (wahrgenommen werden) als andere? Die vorliegende Arbeit adressiert diese unhinterfragte Kreativitätserwartung und stellt sie am Fall der Mode dar.

\subsubsection{Ein Definitionsversuch}

Als »Querschnittsbranchen«, die sich aus unterschiedlichen Wirtschaftszweigen der Produktions-, Dienstleistungs- und Handelssektoren zusammensetzen und die ständig durch neue Wirtschaftszweige und Branchenumstellungen verändert oder erweitert werden, besteht das Feld aus einer Arena heterogener Akteure, die sich in konstantem Positionsgerangel befinden (Fligstein/McAdam 20II). Das Feld der Creative Industries ist dezentral und fragmentiert und deshalb besonders volatil und risikobehaftet (Söndermann/Weckerle 2008: 8; Weckerle/Theler 20Iо: II). Dies hat die Zunahme horizontaler Vernetzung und komplexer Verbindungen unter den Akteuren und elaborierten und erweiterten administrativen Komponenten in den einzelnen Organisationen zur Konsequenz (Scott I994: 2I4). Das Feld ist dynamisch und entwickelt sich 
durch Mitgliederfluktuation kontinuierlich weiter. Als Verband unterschiedlicher und teilweise auch fragmentierter Sektoren sind die Creative Industries trend- und veränderungsanfällig (Scott/Meyer I99I: I35). Die Struktur des Feldes weist Merkmale auf, die an diese Volatilität, Heterogenität und das damit verbundene Risiko angepasst sind. So lässt sich zum Beispiel beobachten, dass eine geringe Arbeitnehmerzahl auf eine vergleichsweise hohe Anzahl an Arbeitsstätten verteilt ist, weshalb zunehmend von »Mikro- bzw. Nanounternehmen « gesprochen wird (Weckerle/Theler 20IO: II). Ein Indiz dafür ist das Bild, welches die Stadt und der Kanton Zürich (Stand 200I) abgeben: Alle Beschäftigten im Bankensektor verteilen sich auf rund 750 Betriebe. Der Anteil der Beschäftigten der Creative Economy entspricht lediglich 78 Prozent der Beschäftigungen im Bankensektor, die sich jedoch auf über 800 Arbeitsstätten verteilen (Weckerle/Söndermann 2005: 3).

Im Vergleich mit >profaneren Industrien ist den Creative Industries weder bekannt, welche Güter sie in ferner Zukunft produzieren werden, noch können sie sich auf Technologien oder Produktionsweisen festlegen. ${ }^{2 \mathrm{I}}$ Die rasante Umwälzung von Ideen und Gütern (Logik der Erneuerung), unklare Nachfrage (die Nachfrage muss oft erst geschaffen werden und Informationen darüber sind zu spät verfügbar), die Abwesenheit von Patentlösungen und Formeln wie auch die Reproduktionshürden (gewisse Güter können nur im Entstehungskontext konsumiert werden ${ }^{22}$ ) verstärken Unsicherheit und Mehrdeutigkeit gegenüber der industriellen Umwelt. Das führt zu einer unsicheren Marktlage, die typisch für Märkte ist, die von Neuerungen geprägt sind (Nelson/ Winter I977; Bechky 2006; Potts/Cunningham 2008; Chen 20I2). Gegenseitiges Beobachten, Imitation und Marktinformation - vor allem von stark sichtbaren und angesehenen Organisationen (Powell/Smith-Doerr 1994: 376) - reduzieren Mehrdeutigkeit und Unsicherheit und unterstützen Bedeutungszuschreibungen in kompetitiven Feldern, wie beispielsweise in den Creative Industries (Anand/Peterson 2000: 27I). Dieser Austausch von und Zugang $\mathrm{zu}$ Information ist weiter ein typisches Feldmerkmal (Powell/DiMaggio I99I) und zentral für die Konstruktion eines Marktes, denn Marktinformation trägt zur Sinnstiftung unter den Feldmitgliedern und deren Umwelt bei (Wooten/ Hoffman 20I3). Dadurch werden Märkte kognitiv (aus der Perspektive kollektiver Akteure) konstruiert und in Kraft gesetzt (Anand/Peterson 2000; Mützel

21 Die Verschiedenheit der Creative Industries wird an fünf Merkmalen festgemacht: der Unternehmens- und Personalstruktur, dem Wachstumspotenzial, der (über-)regionalen Vernetzung verwandter Branchen und der vornehmlich weiblichen Angestellten (Weckerle/Söndermann 2005: 15).

22 | Oft handelt es sich um "Erfahrungs-Güter", das heißt um Produkte, welche an Erlebnisse und Emotionen gebunden sind und dadurch einen intensiven, aktiven Konsumprozess zur Folge haben (Weckerle/Theler 2010: 11). 
20I5). In solchen Märkten befindet sich Kreativität in einem Zustand steter (Weiter-)Entwicklung, wodurch sich die Deutungs- und Handlungsmöglichkeiten vervielfältigen.

Infolge der Abwesenheit einer sicheren Handlungsformel bleibt unklar, wie kreative Ideen umgesetzt werden und wie stark diese Umsetzungen an Traditionen anknüpfen sollen (Jones/Svejenova/Strandgaard Pedersen 20I6). Wiederholt wird argumentiert, dass die Mehrdeutigkeit das Resultat der von Wandel begriffenen Natur der Kulturproduktion und Kreativwirtschaft und einer Instabilität der Bewertungsmuster typisch für Industrien sei, die einen ästhetisch-kreativen Produktionsansatz einem funktionalistisch-nützlichen vorziehen (Dowd 2004; Jones/Thornton 2005; Lant/Hewlin 2008). Der Wert kreativer und kultureller Produkte ist sozial konstruiert und wird durch den Diskurs und die Akzeptanz bestimmter Feldteilnehmer geprägt, nämlich »intermediaries, i. e. people anointed by society as being >knowledgeable< about art - critics, art historians, museum curators - that in turn draws upon and reinforces, in a virtuous cycle, the conventions of value in broader society« (Khaire 20I5: 20I). Typisch für diese Industrien ist es also, dass kulturelle Normen und soziale Akzeptanz ineinandergreifen. Schließlich ist der ökonomische Wert davon abhängig, ob die Gesellschaft diese Güter als angemessen, zulässig und wertvoll auffasst (ebd. 20I4).

Insofern wird nicht nur der soziale, sondern auch der ökonomische Wert der neuen Kreation im Feld konstruiert und darüber entschieden, welche zu »fads« oder »fashions« werden (Hirsch I972: 649). Der Markt kreativer und expressiver Güter wird folglich von einer sozialen Informationslogik bestimmt, die sich von simpler Substitution oder der Bewältigung von Marktversagen abhebt:

The Cls [Creative Industries] rely, to a greater extent that other socio-economic activity, on word of mouth, taste, cultures, and popularity, such that individual choices are dominated by information feedback over social networks rather than innate preferences and price signals. De gustibus non est disputandum is simply not the point, but rather that other people's preferences have commodity status over a social network because novelty by definition carries uncertainty and other people's choices, therefore, carry information. (Potts u. a. 2008: 170)

Feldbeobachtungen und Marktinformation - vor allem anderer Personen - reduzieren die neuheitsimmanente Unsicherheit, welche die Herstellung kreativer Güter in sich trägt. Ein Effekt dieser Unsicherheit und Mehrdeutigkeit ist die Rationalisierung und Standardisierung der Produktions- und Distributionsprozesse sowie die erhöhte Feldbeobachtung (um den Informationsfluss weiter zu steigern). Neue Marktinformation führt zu interner Differenzierung 
und dies zur Entstehung und Erschließung neuer Nischen, die wiederum als Koalitionen den Markt strukturieren (Anand/Peterson 2000: 282).

Andererseits offenbaren sich vorher unvorstellbare Wahlmöglichkeiten. Die Creative Industries werden deshalb als »the domain of the emergence of new choices over things not previously imagined rather than the universal substitution problem between known possibilities« bezeichnet (ebd.: I7I f.). Das Angebot besteht daher nicht aus standardisierten Produkten, sondern der Hervorbringung eines Repertoires neuer Möglichkeiten. Ein bezeichnendes Beispiel stammt aus dem Videospielsektor, in dem die Spezialwünsche einiger >Geeks den konventionelleren Vorstellungen der >Masse< gegenübergestellt werden, wobei bestenfalls alle Nischen bedient werden. Erst durch die Rationalisierung des Produktionsprozesses wurde eine Herausbildung von Teilbereichen und die damit verknüpfte Spezialisierung der Entwicklerteams trotz diversifiziertem Produkteangebot ermöglicht (Tschang 2007: 995 f.).

Standardisierung und Rationalisierung werden als essenzielle Faktoren in der Entstehung und Entwicklung kreativer Güter beschrieben (Gilson u. a. 2005; Tschang 2007; Chen 20I2), denn dadurch werden die zentralen, betrieblichen Prozesse und Strukturen von Umwelteinflüssen wie wechselndem Personal, Publikumsresonanz, Produktionsfluktuation oder auch sich verändernden Modeströmungen abgeschottet (Meyer/Rowan I977; DiMaggio/ Powell I983; Thompson 2008). Feldbeobachtung, Informationsaustausch und eine erhöhte Kommunikationsfrequenz sind weitere Effekte der dynamischen Umwelt. Darunter zählt auch die Feststellung, dass Hersteller in direkten Kontakt mit Konsumenten treten. Als Beispiel hierfür dienen die unzähligen Möglichkeiten der »customization«, einer Strategie, bei der die Massenware an die Bedürfnisse und Wünsche des Individuums angepasst wird und die gemäß Hirsch seit den I980ern der verbreitete Ausdruck »postmoderner Sensibilitäten « ist (2000: 358). Es ist eine Möglichkeit, mit kleinsten Produktionsmengen auf Preissensibilitäten zu reagieren (Porac/Thomas/Baden-Fuller I989: 4II f.).

Schließlich dienen auch organisationale Kognitionen der Orientierung im Feld, vor allem die der kognitiven Abbildung der Umwelt, der Definition der Konkurrenz und der Einrichtung einer Nische, wobei auf der Basis ähnlicher Weltsichten und Deutungsweisen Sinnesgemeinschaften entstehen (Porac/ Thomas I990; Lant/Baum I995). Das organisationale Feld wird sorgfältig gescannt und die Konkurrenz mittels »taxonomic mental models« kategorisiert und eingestuft (Porac/Thomas I990).

Das generierte Wissen dient der Orientierung: Entweder orientieren sich Organisationen an erfolgreichen Modellen oder besetzen Nischen, die nur schwer imitierbar sind (ebd.: 225). Die Konkurrenzdefinition bezieht sich folglich nicht nur auf die meist unvollständigen Industrie- oder Marktdefinitionen, die auf technologische, materielle Transaktionen oder Substituierbarkeit verweisen, sondern basiert maßgebend auf der kognitiven Selbst- und 
Fremdwahrnehmung. Als Beispiel dafür dient ein Artikel über die schottische Strickwarenindustrie (Porac u. a. I989), der beschreibt, wie die schottischen Hersteller hochwertiger Qualitätsbekleidung italienische Modebetriebe oder asiatische Massenware als Konkurrenz ausschließen. Das liegt daran, dass die Hersteller ihre Nische, basierend auf kognitiven Strukturen (Tradition, »general causal beliefs «) und technologischen Wahlalternativen (Unternehmensaktivitäten, Entscheidungsmöglichkeiten), als »qualitativ hochwertige Kaschmirpullover und Strickjacken« bezeichnen. Diese Bezeichnung definiert den Konkurrenzspielraum, die »primary competitive group«, deren Mitglieder die psychologische und soziologische Realität gestalten, die zur Basis wirtschaftlicher Aktivitäten wird. Primärgruppen entstehen durch reziprokes Erlassen materieller und kognitiver Bedingungen. Kognition und Technologie sind deshalb untrennbar.

Because of the interweaving of cognitive structures and technical choices, this tradition locks out competitors and locks in the Scottish firms. Because of the joint action of interwoven technical and cognitive constraints, they have very little choice but to look inward and develop ways of competing among themselves. (Porac u. a. 1989: 410)

Schließlich stabilisieren mentale Modelle und strategische Wahlmöglichkeiten die Marktnische und reproduzieren kognitive Strukturen und technologische Wahlmöglichkeiten. Organisationen, die nicht unter dem Radar dieser Strukturen und Wahlmöglichkeiten fliegen, werden von den Mitgliederorganisationen schlicht nicht als Konkurrenz wahrgenommen.

Folglich ist das Balancieren der Kreativität und Wirtschaftlichkeit eine Folge der Unsicherheitsfiktion und dynamischen Umwelt, deren Bewältigung neue Organisationsformen über die kulturpolitischen, branchentypischen und unternehmerischen Grenzen hinweg erfordert (Scott 2008: 19). Organisationale Felder umspannen interorganisationale, informelle wie auch formale Beziehungen, deren normative Aspekte und kognitive Komponenten zur Grundlage für das kollektive Hervorbringen von Kreativität werden. Die normative Kreativitätsdefinition ist zum »kategorialen Imperativ« im Feld der Creative Industries und seinen Sektoren und Branchen geworden. ${ }^{23}$

Es überrascht kaum, dass sich >nicht kreative< Industrien kreativer Ressourcen bedienen und die erfolgreichen Managementideen der Kreativindustrie und Kulturproduktion in ihre Prozesse implementieren. Ein Beispiel sind

23 | Selznick (1984: 40) spricht von der "infusion with value“. Dieser Wert stattet die Organisation mit einer Aufgabe und einer Kompetenz aus, die dabei hilft, konfligierende und unverständliche Forderungen der externen Umwelt zu bewältigen. Kreativität in den Creative Industries nimmt die Form einer solchen "Wertinfusion" ein. 
Automobilhersteller, welche Designer anheuern, um den Sitzkomfort, das Erscheinungsbild der Karosserie oder die visuelle Vermarktung zu optimieren:

The blurring of boundaries is also leading to wholesale organizational experimentation as far afield as automobiles. Almost all the major car companies, domestic as well as foreign, have design studios in California. Although the design studios are formally owned and funded by the car companies, they are managed separately, often by a charismatic designer-manager, in much the same way that the so called independents are run by the major Hollywood studios, or recording labels are set up by large music groups for highly imaginative record producers. (Lampel u. a. 2008b: 10)

Demzufolge kann von einer >Kreativisierungstendenz $<$ vieler Industriezweige gesprochen werden.

\subsection{Empirische Feldbeschreibungen}

Der relative junge Sektor der Creative Industries, »started as hobbies by enthusiastic amateurs or shunned obsessives, or through unpredicted breakthroughs - in other words, outside established market norms« (Potts u. a. 2008: I78 f.), hat sich zu Subfeldern weiterentwickelt, die sich um die Produktion, Distribution und den Konsum von Neuerungen formiert haben. Die folgenden Auseinandersetzungen sind Beispiele für die Diffusion eines Konzeptes, das stark durch die staatliche Kulturpolitik beeinflusst und betrieben wurde und seit Ende der I99oer-Jahre zuerst als Gegenstand staatlicher Regierungen - die Schweiz hat sich 2003 dem Diskurs angeschlossen - nun auch die Agenden globaler und internationaler NGOs schmückt.

Der Einfluss staatlicher Akteure auf die Entstehung der Creative Industries ist zentral (Scott I994: 2I2 ff. Fligstein/McAdam 20II: I3). Kritische Stimmen äußern sich dazu mit dem Hinweis, dass hier ein Take-over der Kulturpolitik durch die Wirtschaftspolitik stattgefunden hätte, auf der Suche nach einem hippen und coolen Versuch des ökonomischen und sozialen Engineerings (Eikhof in Pratt u. a. 20I5: 245). Durch das Traktandieren nationaler Regierungseinheiten wird die Produktion kreativer Güter zum »object of policy« (DiMaggio I99ı: 275). Empirisch lässt sich dabei beobachten, dass diese Beispiele Zeugen einer konzeptuellen Veränderung sind: Die Feldlogik verändert sich von kumulierten Produktionsstandorten hin zum sozialen Informationsnetzwerk verschiedener Dienstleistungsplattformen (Weckerle/Söndermann 2005). Dieser Wandel in der Selbstbeschreibung ändert auch die Fremdwahrnehmung, weshalb sich die Grenzen zwischen kreativen/nicht kreativen Konsumgüter- und Dienstleistungsindustrien zunehmend auflöst. 


\subsubsection{Die Kulturpolitik der UK Labour Regierung, 1998 und 2001}

Die von der UK-Labour-Regierung im Jahre 1998 gegründete »Creative Industries Taskforce«, eine Einheit des Departments für Kultur, Medien und Sport (Department for Culture, Media and Sports, DCMS), setzt sich mit Fragen der Kulturpolitik auseinander und beschreibt im »Creative Industries Mapping Document« Industriesektoren, die in Bezug zu Kreation, Kunst und Kulturprodukten stehen. Das Dokument wurde im Jahr 200I überarbeitet und definiert die Kreativwirtschaft als »industries which have their origin in individual creativity, skill and talent and which have a potential for wealth and job creation through the generation and exploitation of intellectual property« (DCMS 200I: 5). Das Dokument bezeichnet I3 industrielle Sektoren als Teil der Creative Industries, unter welchen sich nicht nur die klassischen Künste wie Architektur, Kunst, (Kunst-)Handwerk, darstellende Künste, Film und Video oder Musik befinden, sondern auch mehr kommerziell ausgerichtete Sektoren, so wie (Mode-)Design, das Verlagswesen, die Werbebranche, (interaktive Freizeit-)Software oder Computerdienstleistungen und Fernseh- und Radiosender.

Mit dem Begriff der "creative industries" wurde die Hybridisierung von kommerzieller kultureller Produktion sowie von Produktion, die aus ökonomischer Sicht auf Grund von Marktversagen als subventionswürdig galt, was für die traditionellen Künste von bildender Kunst bis zu Literatur zutrifft, in eine ökonomische politische Agenda eingebunden. (Wuggenig 2016: 45)

Die Konjunktur des Begriffs im englischsprachigen Raum ist die Folge des $>$ Rebrandings< der Labour Partei der I99oer- und 200oer-Jahre. Auf dieser Basis hat sich eine Industriewelt entwickelt, die in der Wissensgesellschaft einen großen Teil der Arbeitnehmer beschäftigt und deshalb zu den mächtigsten und wichtigsten Industriesektoren der letzten zwei Jahrzehnte zählt (Florida 2006; Krämer 20I4; Reckwitz 20I4).

Obwohl die Agenda der Labourpartei die scheinbare Absicht mitbringt, Kultur und Kunst zu demokratisieren - denn Kreativität und Kultur sollen zugänglich gemacht und gleichgestellt wie andere Industrie- und Gesellschaftsbereiche gefördert werden -, warnt McRobbie (20II) vor einigen Missständen, die diese Arbeitstransformation durch totale Kreativitätsforcierung mit sich bringt: Die Betonung freiberuflicher Tätigkeiten ignoriert alte Arbeitsplatzbestimmungen, was oft zu einer negativen Auslegung oder gar Untergrabung grundlegender Rechte und Pflichten am Arbeitsplatz führt. Weiter fällt ihr eine verdeckte Normalisierung der Unterbeschäftigung auf, die einerseits aufgrund ständiger, zeitintensiver Selbstbewerbung, aber auch der Fluktuationen und Zeithaftigkeit von Projektarbeit, »a frenzy of activity, projects and excite- 
ment which distracts attention away from the downtime between projects«, nicht zur Kenntnis genommen wird (McRobbie 20Ir: 33).

Dieses Selbst- und Fremdverständnis entwickelt sich weiter zur Basis weiterführender Diskussionen und Debatten im Feld der ehemaligen Kulturindustrien und erweitert diese um eine Mehrzahl an Kreativindustrien. Und auch vor dem wissenschaftlichen Diskurs macht das Konzept keinen Halt: Ab Ende der I990er-Jahre und zu Beginn der 200oer bestärkt das Interesse die Verbreitung verwandter Themen, und die Anzahl akademischer Artikel rund um Kreativität explodiert regelrecht. In dieser Debatte wird der Kreativitätsbegriff als affirmative Selbstbeschreibung geführt.

\subsubsection{Die Creative Economies in der Schweiz}

Im Jahr 2003 legt sich das Europäische Parlament auf eine kulturpolitische und rechtliche Definition des Begriffs »Cultural Industries « fest und beginnt sich damit auch mit der »Kreativwirtschaft«, deren Stärkung der Wettbewerbsfähigkeit und der kulturellen Vielfalt auseinanderzusetzen. ${ }^{24}$ Im selben Jahr wird von der Zürcher Hochschule der Künste $(\mathrm{ZHdK})^{25}$ der erste umfassende Bericht zur Kulturwirtschaft in der Schweiz vorgelegt (Weckerle/Söndermann/ Hofecker 2003). ${ }^{26}$ Seit dem ersten Kulturwirtschaftsbericht Schweiz hat sich der Begriff von Kultur- und Kreativwirtschaft in »Creative Economies « - alles Synonyme für die Bezeichnung »Creative Industries« - transformiert, eine Veränderung, die mit der globalen Ausrichtung dieser Sektoren begründet wird. In den Jahren 2005, 2008 und 2010 wurden von der Stadtentwicklung und Wirtschaftsförderung der Stadt resp. der Standortförderung des Kantons Zürich drei Kreativwirtschaftsberichte in Auftrag gegeben und von der ZHdK verfasst. Die Berichte entschlüsseln die Arbeitsmodelle der Kreativwirtschaft als integrative Modelle, die sich an der Schnittstelle von Staat, Gesellschaft und Wirtschaft verorten lassen und diese Bereiche zunehmend überbrücken. Mit Überlegungen zu Mikrofinanzierung und Marktzutritt, regulativen Rahmenbedingungen und geistigem Eigentum und dem möglichen Transferpotenzial von Wissen und Ressourcen sind diese Berichte an der Schnittstelle der kulturpolitischen Subsidiaritätsdiskussion und der industriepolitischen Debatte um die Erwerbswirtschaft kreativer und kultureller Betriebe zu verorten (Weckerle/Söndermann 2005; Weckerle/Theler 20I0). Des Weiteren hat sich ein Dachverband Kreativwirtschaft Schweiz formiert, der diese als »breites und poten-

24 | Zugriff am 28. Mai 2019 auf www.europarl.europa.eu/sides/getDoc.do?pubRef $=-/ /$ EP $/ /$ TEXT+TA+P5-TA-2003-0382+0+D0C+XML+VOs//EN.

25 | Ehemals Hochschule für Gestaltung und Kunst Zürich (HGKZ).

26 | Zugriff am 28. Mai 2019 auf www.yumpu.com/de/document/view/19446980/ erster-kulturwirtschaftsbericht-ch-hgkz-2003-buchlobby-schweiz/5. 
tes Rückgrat der Schweizer Volkswirtschaft« bezeichnet und das Ziel verfolgt, den Betrieben der Schweizer Kreativwirtschaft eine »gemeinsame, politische Stimme zu geben. ${ }^{27}$

Der jüngste Bericht der ZHdK von 2016 nimmt ein Relabeling der Feldbezeichnung von Kreativwirtschaft in Creative Economies vor (Weckerle/Page/ Grand 20I6). Die Veränderung in der Rhetorik ist auffällig: einerseits, weil der Bericht nun in Webseitenform digital und bunt illustriert zugänglich ist, andererseits, weil es sowohl eine deutsche wie auch eine englische Version (digital und physisch) gibt. Die Dokumente richten sich zwar an die nationale Verwaltung und Politik und bieten eine empirisch-quantitative Grundlage zur hiesigen Weiterführung der Diskussion, knüpfen aber gleichzeitig an die in anderen europäischen Städten geführten Diskurse an und gewinnen zunehmend international an Bedeutung. Die Berichte betonen den wirtschaftlichen (Standort-)Vorteil, den es rechtlich zu schützen und politisch zu begrüßen gilt, der durch Kreativwirtschaft entsteht:

Kreativwirtschaft fokussiert auf die Kreativbetriebe im privatwirtschaftlichen Sektor. Es sind dies Unternehmen/r, die sich auf erwerbswirtschaftlicher Basis mit der künstlerischen/kreativen Produktion, ihrer Vermittlung und/oder Verbreitung von entsprechenden Gütern und Dienstleistungen befassen. (Weckerle/Page/Grand 2016)

Die Definition bezieht sich auf »kulturbezogene Wirtschaftszweige« (2005: Ib: 9), einem Branchenkomplex, der aus I3 Teilmärkten und deren wechselhaften Branchenzweigen besteht, wobei die Grenzziehung und Definition rein illustrativer Natur sei (Weckerle/Theler 20IO: I2). Mit kulturbezogenen Wirtschaftszweigen sind die Musikwirtschaft (Komponisten, Musiker, Musiklehrer, Toningenieure, Interpreten, Musikensembles), der Buchmarkt (Schriftsteller und Autoren), der Kunstmarkt (Bildende Künstler, Restauratoren, Kunstlehrer), die Filmwirtschaft (Drehbuchautoren, Filmschauspieler, Filmproduzenten), die Rundfunkwirtschaft (Moderatoren, Sprecher, Produzenten), der Markt der darstellenden Künste (darstellende Künstler, Artisten, Tänzer, Kabarettisten), die Designwirtschaft (Designer, Gestalter, angewandte Künstler), der Architekturmarkt (Architekten und Landschaftsplaner), die Softwareund Gamesindustrie (Software- und Gamesentwickler), das Kunsthandwerk (Kunsthandwerker, Gold- und Silberschmiede), der Pressemarkt (Journalisten und Wortproduzenten) und schließlich der fonotechnische Markt (Hersteller und Händler film-, rundfunk- und fonotechnischer Geräte) gemeint. In diesem Verständnis werden »die unterschiedlichen Akteure als Teil eines heterogenen, verteilten, sich immer wieder neuformierenden Wertschöpfungspro-

27 | Zugriff am 28. Mai 2019 auf https://kreativwirtschaft.ch. 
zesses betrachtet. Wertschöpfung wird damit jenseits ökonomischer Relevanz neu definiert und reflektiert« (Weckerle u. a. 20I6: 4). Wertschöpfung wird folglich als kollektives Phänomen soziologisch neu definiert.

Für das spezifische Interesse dieser Arbeit am Modesektor ist besonders informativ, dass der Teilmarkt der Designindustrie (die quantitativ bedeutendste Kreativindustrie Zürichs) weder Textildesign, Modedesign noch Kostümbildung umfasst. Während Kostümbildung als Teil der »darstellenden Künste«, d. h. dem »Betrieb von Kultur- und Unterhaltungseinrichtungen resp. Theater- und Ballettgruppen«, zugeordnet werden könnte (Weckerle/Theler 20ıо: 29), so scheinen Mode- und Kleidungs- wie auch das Textildesign gänzlich ignoriert $\mathrm{zu}$ werden. ${ }^{28} \mathrm{Im}$ jüngsten Bericht wird teilweise darauf eingegangen: Während Modedesign in Grafiken als Teil der Designwirtschaft neben Produkte- und Grafikdesign vorkommt, wird im Marktporträt zur Designwirtschaft nicht weiter auf die Modebranche eingegangen. Gemäß den Schweizer Berichten ist Mode schlicht nicht auf dem Radar der Kreativwirtschaft.

Seit 200I hat der prozentuale Anteil der Kreativwirtschaft am Bruttoinlandsprodukt der Schweiz von drei Prozent auf rund vier Prozent zugenommen. ${ }^{29}$ Ebenso verzeichnet der Anteil der in der Kreativwirtschaft ansässigen Betriebe der Schweiz eine kontinuierliche Zunahme, die sich zurzeit bei knapp elf Prozent befindet; und 5,5 Prozent aller Beschäftigten in der Schweiz erbringen ihre Leistung in einem der Teilmärkte der Kreativwirtschaft (Weckerle u. a. 20I6: 9; Weckerle/Theler 20Iо: 6). Zürich wird dabei als Zentrum der Kreativwirtschaft Schweiz dargestellt, denn 5,9 Prozent des BIP des Kantons Zürich entstammen der Kreativwirtschaft (ebd.: 6). Dabei ist der Anteil der Arbeitsstätten, der Beschäftigten wie auch des Gesamtumsatzes im Schweizer Vergleich, der sich für den Kanton Zürich bei rund einem Viertel beläuft, bemerkenswert (ebd.: I6). Der Bericht zur Kreativwirtschaft in Zürich hebt vor allem die Standorte, deren Vorteile und Förderung hervor und betont Zürichs bedeutende Rolle.

28 | Der einzige Bezug zur Bekleidungsindustrie wird in der Darstellung der Wertschöpfungskette respektive der intern angegliederten oder extern zugezogenen Designdienstleistungen am Beispiel der "Funktionsbekleidung" gemacht, wobei dieser Verweis im Kontext der absoluten Ignoranz gegenüber jeglicher Art textiler Güter ziemlich deplatziert scheint (Weckerle/Söndermann 2005: 45).

29 | Anteil an BIP: 2001: 3,1 Prozent; 2008: 4,2 Prozent; 2013: 3,9 Prozent; Betriebsanteil: 2001: 8,7 Prozent; 2008: 10 Prozent; 2013: 10,9 Prozent; Beschäftigtenanteil: 2001: 3,9 Prozent; 2008: 5 Prozent; 2013: 5,5 Prozent (Weckerle/Söndermann 2005; Weckerle/Theler 2010; Weckerle u. a. 2016). 


\subsubsection{Die Definition der UNCTAD}

2008 publiziert die UNCTAD (United Nation Conference on Trade and Development) den Creative Economy Report, der die Creative Industries als deren Kern beschreibt, der für die Kreativität das essenzielle Merkmal ist (UNCTAD 2008a ).$^{\circ}$ Dadurch wird Kreativität nicht nur zum globalen Problem der Entwicklungspolitik, denn die Creative Industries werden zur Möglichkeit für wirtschaftlichen Aufschwung in weniger entwickelten Ländern, sondern wird auch für die WIPO (World Intellectual Property Organization) von Interesse, wodurch man sich eine Stärkung und Verschärfung der rechtlichen Sachlage erhofft. Die Globalisierung des Gegenstands offenbart sich am behördenübergreifenden Interesse vieler Agenten wie beispielsweise die UNCTAD, UNDP, UNESCO, WIPO oder auch ITC, die sich am Bestreben der Definierung und rechtlichen Grundlage für die Creative Industries beteiligen. ${ }^{3 \mathrm{I}}$

Inhalt dieser Bestrebungen ist die durch die Digitalisierung zunehmende Veränderung der Marktwirtschaft, vor allem dieser Teile, deren Inhalte kultureller oder kreativer Natur sind. Der Advent neuer Informations- und Kommunikationstechnologien, die neuen Medien, ermöglichten sowohl neue Distributionskanäle wie auch neue Inhalte (beispielsweise Webdesign, Softwareentwicklung, Videogames). Als »vast and heterogeneous field dealing with the interplay of various creative activities « (UNCTAD 2008b: 13) verknüpften die Creative Industries Kultur, Wirtschaft und Technologie und reagierten mit neuen Formen der Produktion, Distribution und des Konsums auf die erhöhte Nachfrage. Die UNCTAD definiert sie wie folgt:

"Creative Industries" can be defined as the cycles of creation, production and distribution of goods and services that use creativity and intellectual capital as primary inputs. (Ebd.: 13)

\section{Der Bericht fährt fort:}

In the contemporary world, creativity is often referred to as a key resource in the knowledge economy, leading to innovation and technological change and conferring competitive advantage on businesses and national economies. The association of creativity with commodities gives rise to a class of products known as ,creative goods and servicesı, of which, many believe, 'cultural goods and servicesı constitute a sub-

30 | Kreative Arbeit findet auch in anderen Industrien oder Branchen mit betriebswirtschaftlicher Ausrichtung statt, so z. B. in der Pharmaindustrie oder im Maschinenbau. Folglich umfassen die Creative Economies ein breiteres Sektorenspektrum als die Creative Industries.

31 | UNDP: United Nations Development Programme, UNESCO: United Nations Educational, Scientific and Cultural Organization, ITC: International Trade Centre. 
set formed by products with artistic for cultural as well as creative content. This in turn enables the definition of ,creative and cultural industries, as those industries producing creative and cultural goods respectively. (Ebd.: 21)

Unter die in den Creative Industries hergestellten Güter fallen Produkte als auch Dienstleistungen mit künstlerisch-kulturellem oder kreativem Inhalt. Die Mode wird (neben der Musikindustrie und Handwerksgütern) als Geschäftszweig genannt, der die wirtschaftliche Einnahmequelle mit der Weiterentwicklung authentischer, kultureller Symbole und Tradition potenziert (ebd.: 23).

Der UNCTAD-Bericht hebt die technologischen Veränderungen in der Gesellschaft der I99oer-Jahre als Entstehungsgrundlage der Creative Industries hervor. Das intellektuelle Kapital wird zur inhaltlichen Basis der Wertschöpfung der Creative Industries, weshalb jenes von zunehmender Wichtigkeit ist und als besonders schützenswert dargestellt wird. In der kulturellen Vielfalt und dem kreativen Talent wird das Potenzial für weiteren Fortschritt von Nationalstaaten gesehen. Eine überarbeitete Version des Berichts wird 20го veröffentlicht. In Anschluss daran lancieren 20I3 auch die UNESCO und die UNDP einen Creative Economy Report in Zusammenarbeit mit dem Fokus auf nachhaltiges Wachstum und Entwicklung.

\subsubsection{Die kulturpolitische Definition der Mode}

Die Berichte sind sich uneinig, ob die Modebranche als Teilmarkt der Creative Industries zu deuten ist. Das UK-DCMS-Modell behandelt die Modebranche gleich wie alle Teilmärkte. Die von der UNCTAD zusammengestellte Übersicht verschiedener Ansätze, die später auch vom Schweizer Bericht und dem UNESCO-Bericht übernommen wird, stellt Mode unterschiedlich dar. Im symbolischen Textmodell wird Mode als »borderline cultural industry« gedeutet, ebenso die Konsumelektronik oder Sportutensilien. Das Modell konzentrischer Kreise begreift Mode als »related industry« neben Werbung, Architektur und Design. Der Copyright-Ansatz der WIPO beschäftigt sich als »partial copyright industries « mit Mode, so wie Spielzeug, Kleidung und Schuhe oder auch Haushaltswaren. Gemäß UNESCO-Statistik wird Mode nicht explizit genannt, ebenso in der Definition der »American for the Arts« (2013: 22). Diese ungenauen Bestimmungen und Deutungsweisen, welche die Mode in der Peripherie der Creative Industries verorten, machen die Mode zu einem besonders interessanten Fall. In der vorliegenden Arbeit wird die Modebranche aufgrund der Spezifizität der Kombination von ökonomischer Orientierung und symbolischer Wertproduktion als Sektor der Creative Industries behandelt. 


\subsection{Der Modesektor}

Seit jeher wurde Mode und Kunst ein enges Verhältnis zugeteilt (Ziege 20II; Titton 20I2). Die gängige Assoziation der Modebranche mit Kreativität rührt nicht zuletzt daher, dass Modedesign an Kunsthochschulen gelehrt wird. Eine Folge davon ist die unhinterfragte Kreativitätserwartung; das heißt, dass Kreativität von der Umwelt erwartet wird. Während beispielsweise in Forschungsund Entwicklungseinheiten vordefiniert ist, wie Neuerungen entstehen und sich ermitteln lassen (z. B. über Patente), besteht im Modesektor keine geteilte Auffassung darüber: »Participants chose the specific topic and materials to use, but they did not have a choice in whether or not they wished to create the art form« (Unsworth 200I: 292). Schließlich bleiben die Kreativitätserwartungen aus dem Feld der einzige Katalysator kreativer Leistungen (Ford I996).

Die Creative Industries bestehen aus einer Vielzahl von Subfeldern, die sich in Sektoren unterteilen. Eines davon ist die Mode. Das Feld der Mode besteht aus einer Vielzahl von Sektoren, so z. B. der Haute Couture, einem von der »Chambre Syndicale de la Haute Couture« geschützten Marktsegment, der Sport- oder Outdoorbekleidung, den Designbrands, dem Modelmarkt oder der Modepresse. Ein Sektor - Scott und Meyer sprechen von »societal sector«, um die soziale Ausbreitung von lokalen Besonderheiten bis zu internationalen Verbindungen zu betonen - wird zu

(1) a collection of organizations operating in the same domain, as identified by the similarity of their services, products or functions, (2) together with those organizations that critically influence the performance of the focal organizations: for example, major suppliers and customers, owners and regulators, funding sources and competitors. (1991: 117)

Während das Feld eine größere Anzahl differenzierter Akteure umfasst, so sind die Mitglieder im Sektor funktional und nicht geografisch äquivalent. Mithilfe der kulturellen Wertschöpfung definieren Aspers und Godart Mode als »multiple networks that connect an upstream of suppliers to a downstream of customers through a market interface made of producers « (20I3: I8I).

Die Modebranche ist ein Sonderfall der Creative Industries, denn sie umfasst sowohl die Aufrechterhaltung traditioneller Handwerkskunst als auch die Einführung neuer Technologien; sowohl die Bewahrung des Vergangen als auch die Förderung des Neuen; sowohl die Einzelanfertigung als auch die Massenproduktion; sie trägt zur Bereicherung kultureller Symbole bei und fixiert technisch-funktionalistische Bedingungen; und schließlich verweist die Mode auf zweierlei Verwendungen des Entwurfs, der Teil des vorgelagerten Entwicklungsprozesses ist, denn sie versteht den Prototypen sowohl als Produkt (beispielsweise in der Haute Couture, außerhalb des Modesektors in der Archi- 
tektur) als auch als Muster, auf welches die Produktion dessen folgt (in der Konfektion, außerhalb des Modesektors beispielsweise im Industriedesign). Die Logik der Haute Couture reklamiert Einzigartigkeit durch Werkstattcharakter, wobei Bekanntes, Normen und Konventionen teilweise absichtlich ignoriert werden. Dadurch wird eine höhere Chance erzielt, radikale Änderungen im Modedesign zu bewirken. Die serielle Herstellung der Konfektionsmode konzentriert auf Modularität und Pluralität im Modedesign - also auf Repertoire statt Risiko (Potts u. a. 2008: I7I) - und erzeugt inkrementellen Wandel (Djelic/Ainamo 2005: 76) bzw. »inkrementelle Kreativität« (Shalley/Gilson 20I7: 606 f.) $)^{32}$ Was >erfolgreiche Mode ausmacht, ist daher sektoren- respektive marktabhängig. Von einem kommerziellen Standpunkt aus lässt sich feststellen, dass gute Mode erfolgreich diffundiert. Reichweite und Schnelligkeit sind mögliche Parameter erfolgreicher Mode. Mode(n) sind dann Übersetzungen von Ideen und Trends, die global diffundieren und in lokale Darstellungen übersetzt werden (Czarniawska/Joerges I996; Czarniawska/Sevón I996b; Sahlin-Andersson I996; Sahlin/Wedlin 2013). ${ }^{33}$ Blumer schreibt dazu: »The dress designers were engaged in translating themes from these areas [the fine arts, recent literature, political debates and happenings, and discourses in the sophisticated world] and media into dress designs « (Blumer I969: 279). Durch die stilistische Modifikation des Designs wird lokale Passung erzielt. Diese betrifft nicht nur technologische oder kulturelle Ansprüche, sondern auch Passung mit symbolischen Werten und Bedeutungszuschreibungen. Konvergieren diese Modifikationen, kann von einer erfolgreichen >stilistischen Innovation<, einem Fortschritt im Modedesign wie beispielsweise das »kleine Schwarze« von Coco Chanel oder der von Christian Dior geprägte New Look, gesprochen werden (Cappetta/Cillo/Ponti 2006). Gleichzeitig liegt darin das Hauptinteresse wissenschaftlicher Erhebungen:

Firms that produce fashionable clothing represent a particularly interesting case for the study of cultural production for two reasons: (1) the necessity to innovate by altering the symbolic values attached to styles of clothing. Several times each year new collections must be created which are expected to combine elements of previous

32 | Als Beispiel inkrementellen Wandels in der Mode werden Khaires empirische Studien zur Modernisierung der Kleidertraditionen in Indien genannt. Khaire beobachtet, wie sich die indische Kleidertradition langsam an globale Moden und Trends und deren Stil in Formsprache und Darstellungsweise annähert und diese schließlich übernimmt (Khaire/Hall 2016; Khaire 2015).

33 | Die neoinstitutionalistische Translationstheorie, die vor allem die skandinavische Lesart geprägt hat und von Czarniawska, Sahlin und Wedlin entwickelt wurde, ist stark inspiriert von den Grundprinzipien der französischen Wissenschafts- und Techniksoziologie Callons, Latours und Tardes. 
styles with new and different ideas; and (2) the necessity to produce clothes whose symbolic values resonate with those of consumers [...]. (Crane/Bovone 2006: 321)

Die Feldmitglieder nehmen aktiv am Prozess konzeptueller Modifikationen, stilistischer Interpretationen und kulturell-symbolischer Sinnzuschreibungen teil und gestalten durch Interaktion und Verhandlungen ein organisationales Feld (Petkova 2oi6).

Als Mitglied des organisationalen Feldes der Creative Industries charakterisieren zwei zentrale Merkmale die Organisationsstruktur und die Besonderheit der Wertschöpfung von Modefirmen. Erstens treffen Modefirmen auf besondere Marktbedingungen (volatile und schwer voraussagbare Nachfrage, Erneuerungsdruck oder Kurzlebigkeit der Konsumgüter), was Klein(st)unternehmen begünstigt, die sektoren- und feldintern in Projekten kooperieren und Aktivitäten koordinieren. Dies hat bestimmte Erwerbsstrukturen zur Folge wie z. B. Kurzzeitverträge oder das Auslagern bestimmter Aufgaben. Weiter reagieren Modefirmen mit Diversifizierung des Angebots und mit Nischenbildung auf die Mehrdeutigkeit im Markt (Djelic/Ainamo I999; Crane 20I2). Zweitens bestimmen spezifische Modalitäten den Wert kreativer Güter. Die Wertschöpfung dieser findet standardisiert mittels iterativer Handlungsmuster und entlang einer Kette von spezialisierten Technologien statt, wobei durch die Umgestaltung und Kombination verschiedener - auch bewährter - Elemente und durch die kommunikative Darstellung symbolischer Wert generiert wird. Die nächsten Kapitel widmen sich diesen Besonderheiten.

\subsubsection{Strukturelle Aspekte}

Strukturmerkmale der Modebranche, die in der Literatur erwähnt werden, sind die kategoriale Unterscheidung zwischen Zentrum und Peripherie und die damit verknüpfte Verortung in den Creative Industries (Zentrum: Literatur, Kunst, Werbung; Peripherie: Mode); die Hierarchisierung und Ermittlung des Zentrums bzw. des Einflusses von Organisationen auf andere Feldmitglieder (Zentrum: Haute Couture, High Street Fashion/Konzerne, New Verticals wie H\&M oder Zara; Peripherie: unabhängige Designlabels); die erhöhte Kommunikationsdichte im Kooperationsnetzwerk der Produktion von Modekollektionen, folglich die Zunahme des Informationsaustauschs unter diesen Kooperationsakteuren, die gegenseitige Wahrnehmung der Akteure; sowie eine geteilte Rationalität, die ähnliche Situationsinterpretationen, Aufmerksamkeitsschemata und Identitäten bedingt. Die kollektive Rationalität bezieht sich auf die symbolische Herstellung, kurze Zyklen des auf Zeit angelegten Geschäfts und die kundenindividuelle Massenfertigung, womit sich der Mode- 
sektor von anderen kreativen Sektoren oder Produktionsindustrien unterschei$\operatorname{det}$ (Djelic/Ainamo 2005). 34

Eine Spezifität des Feldes der Creative Industries ist die Fragmentierung der Kunst- und Handwerksindustrien, die sich in Dachorganisationen (Handwerksverbänden, Produktionsnetzwerken) formieren und für kleinere Organisationen und deren Interessen einsetzen. Die Modebranche ist dafür bekannt, dass sie zumeist aus disparaten, kleinen Handwerksbetrieben besteht (Powell I990; Powell/Smith-Doerr 1994). Das Design wird zwar vom kreativen Zentrum aus koordiniert, die Produktion findet dennoch in interorganisationalen Netzwerken statt. Während größere Konzerne und die sogenannte New Verticals für ihre Unternehmensgröße bekannt sind, machen diese einen marginalen Bestandteil der Arbeitsstätten im Modesektor aus. Die Zerstückelung der Unternehmenslandschaft führt dazu, dass Kleinstunternehmen ein außergewöhnlich hohes wirtschaftliches Risiko auf sich nehmen müssen, um den dynamisch-volatilen Marktverhältnissen (und den größeren, mächtigeren Konzernen) entgegenzutreten. Diese Bedingungen begünstigen die Entstehung kleiner Firmen mit wenig Angestellten und wenig Investitionskapital, die dennoch einen offensichtlichen Vorteil im Kreativmarkt haben, denn sie wägen den Nutzen der Geschäftschancen und das wettbewerbliche Leistungsvermögen stärker ab und fällen so tendenziell treffsichere Entscheidungen (Crane 20I2: 82).

Der Schweizer Modemarkt verbindet das italienische Modell der Modeproduktion mit dem amerikanischen: Das italienische Modesystem ist für die große Diversität der vielen, kleinen Unternehmen und deren Lokalität bekannt, der sowohl hohe Qualität wie auch Kreativität zugeschrieben wird (Crane/ Bovone 2006; Mora 2006). Trotz der Fragmentierung des Sektors handelt es sich um ein integriertes, dezentralisiertes System der Modeproduktion. Dem amerikanischen Unternehmensmodell liegt ein Konzept zugrunde, an das die Produktionsentwicklung und die Produktlinien wie auch die Marketingstrategien angepasst werden. Diese konzeptuelle Kanalisierung und folglich die damit einhergehende lose Kopplung von Produktion und Konzept haben eine Modularität zur Folge, die rasche, systematische Neuerungen ermöglichen und Raum für das Spiel mit dem Detail lassen (Djelic/Ainamo I999: 632). Lose Kopplung trägt somit auch zum Verständnis von Heterogenitäten und Wandel in Organisationen und Feldern bei (Hasse/Krücken 20I4; Schneiberg/ Clemens 2006).

Weiter fördern solche Bedingungen unterschiedliche Organisationsformen, die von permanenten, formalen Unternehmen bis hin zu temporären Projektorganisationen reichen. Die Erwerbsstrukturen erstrecken sich ebenso

34 | Diese Charakteristika sind zwar typisch für viele der Creative Industries, sind in dieser Konstellation jedoch insbesondere als Ausprägungen des Modesektors gelistet. 
von permanenten bis hin zu temporären Anstellungsformen. Damit ist gemeint, dass »kurze und befristete Arbeitsverträge, unregelmäßige Arbeitszeiten und ein tiefes, unregelmäßiges Einkommen im Verhältnis zur beruflichen Qualifikation« die Regel sind (Weckerle/Theler 20Iо: II). So werden formale und informale Verträge über Organisationsgrenzen geschlossen, die oft auch Freelancer und deren Partner inkludieren (Hirsch 2000: 358). Im Verlauf der Karriere einer Kollektion wiederholt sich die Entstehung und Beendigung temporärer Projektaufgaben und -teams, die dann für begrenzte Zeit bestehen und eine Qualitätsveränderung enthalten, die oft in Form eines Entwurfs (Skizze, Stoffdrucke, Prototyp) im Designteam präsentiert wird (s. Kap. 6.3). Als Folge der Einhaltung bestimmter Konventionen und der Kontrolle organisationsspezifischer Aspekte wie Finanz- oder Zeitdimensionen betonen Modefirmen die künstlerisch-kreativen Prozesse anhand jener neue Produkte hergestellt werden (Crane/Bovone 2006; Aage/Belussi 2008; Godart/Mears 2009; Aspers/ Godart 20I3).

Die im Projekt hergestellten Güter und das dabei generierte Wissen werden nicht nur intraorganisational in Archiven oder im Personal gespeichert, sondern werden über die Akteure wieder zurück ins organisationale Feld gespeist, das für die Produktion, die Distribution und den Konsum der Güter wie auch des Wissens zuständig ist (Sydow/Lindkvist/DeFillippi 2004; Godart u. a. 20I4). Durch diese netzwerkbasierten, projektartigen Organisationsformen wurde eine Möglichkeit der regelmäßigen und systematischen Neuheitsexploration geschaffen, die auf das vermeintliche Tempo der Erneuerung in der Modeindustrie reagiert (DeFillippi 20I5; Bakker u. a. 20I6). Aus diesen Gründen begünstigen die Marktstrukturen der Creative Industries Projekte als typische Organisationsform, wobei das Feld als Netzwerk fungiert, welches die Produktion und Kooperationen koordiniert (DeFillippi/Arthur I998; Grabher 200I; Hargadon/Bechky 2006; Perretti/Negro 2007; Obstfeld 2012).

\subsubsection{Modalitäten der Produktion}

Veranlasst durch die schnelle, serielle Herstellung, werden die Produktionsund Vermarktungsprozesse standardisiert. In der Folge können Ressourcen einfacher geplant und Handelsbeziehungen zuverlässiger koordiniert werden. Die zentrale Schwierigkeit für das Management in der Mode ist es, die Balance zwischen serieller Herstellung und handwerklichem Kreieren unter der Bedingung ständiger Erneuerung zu halten. Aufgrund der Rationalität der Erneuerung ist Neuheit der qualitative Mehrwert in der Mode. Die Mode schafft es wie kaum ein anderer Sektor, Geschmacks- und Erwartungsbildung durch Kommunikation und Marketing zu stimulieren und dann mit spezifischen Produkten und Dienstleistungen zu bedienen (Lampel/Shamsie/Lant 2008c: 295). Anstelle eines funktionalen Substituts tritt die stilistische Neuerung, die 
sowohl die soziale Bedeutung als auch die ästhetische Gestalt ändert. Das Feld der Mode »represents the context with the longest life span where stylistic innovation is the core of the offering « (Cappetta/Cillo/Ponti 2006: I280). Somit werden Vorstellung und Erwartungen von Kreativität vorgängig installiert und immer wieder bestätigt.

Die Beschaffenheit kreativer Güter erstreckt sich entlang eines Kontinuums von totaler Virtuosität bis zu absoluter, ökonomischer Effizienz, wobei beide Extreme zugunsten der Verständlichkeit und Verwendbarkeit von Designern vermieden werden (Tschang 2007: 994). Deshalb wird der Wert der Mode über Rekombination und Wiederholung gewisser Elemente generiert, die den kreativen Erfolg stark beeinflussen. Hierbei wird auch von »imitating success « gesprochen, denn imitiert wird - zumeist nur partiell -, was als erfolgreich gilt (Sahlin-Andersson 1996; Sevón 1996).

Prozesse der Mode adressieren zwei Seiten derselben Medaille, wenn sie Kontraste wie Erneuerung und Tradition, Betriebszuverlässigkeit und Entdeckungsglück, Auftragsarbeit und Künstlerfreiheit in sich vereinen. Das Kombinieren und Wiederholen begünstigt schließlich Modifikationen und Sowohl-als-auch-Lösungen, die eine modespezifische Form der Erneuerung darstellen. Auf der Basis von Routinen und Wiederholungen werden Stilelemente kombiniert und Kontingenzen reduziert. Ebenso wird damit die Grundlage für Improvisation und Variation gelegt (Mora 2006: 340; Chen 20I2: 632). Bekanntes, wie z. B. Routine (Kap. 6.I), dient als Repertoire und Inspirationsquelle für neue Ideen, kann jedoch auch zu ähnlichen Umsetzungen führen. Gleichzeitig stellt die Modifikation des Bekannten Verständlichkeit her (Blumer I969; Becker 1974; Lampel/Shamsie/Lant 2008a).

Dennoch suchen Organisationen nach Originalität und Unverwechselbarkeit und loten ihre Andersartigkeit stets neu aus (Lampel u. a. 2000: 266; Lampel 2008: 54), denn offensichtliches Kopieren ist negativ konnotiert und kann als Diebstahl von geistigem Eigentum rechtlich verfolgt werden. Es entstehen Spannungen zwischen Gleich- und Anderssein, die es strategisch auszugleichen gilt. Strategische Ähnlichkeit (»range of acceptability«) entsteht, wenn Firmen Verschiedenheit und Konformitätsdruck ausgleichen (Deephouse 1999). Dabei gilt es, die Ähnlichkeit zu anderen zu wahren, um Vergleichbarkeit und so Legitimität zu erhalten, während Verschiedenheit zu anderen postuliert wird, um die Unvergleichbarkeit zu unterstreichen und Wettbewerb $\mathrm{zu}$ unterbinden (van Werven/Bouwmeester/Cornelissen 20I5). Kreativität in der Mode ist dann erfolgreich, wenn sie die Neuerung nuanciert und gleichzeitig die Tradition - den vestimentären Stil - wahrt: »Consumers need familiarity to understand what they are offered, but they need novelty to enjoy it« (Lampel/Lant/Shamsie 2000: 264). Ideen diffundieren dann erfolgreich, wenn sie rezipiert werden, d. h., als »attraktiv« bezeichnet werden: »Issues that are perceived as new and extraordinary, but not too different from what 
is generally accepted - issues that thus fit societal genres - attract attention « (Sahlin-Andersson r996: 87). Je besser und attraktiver die Idee, desto weiter deren Reichweite.

Geht es um Kreativität, ist Erfolg ein Äquivalent für soziale Akzeptanz (Csíkszentmihályi ı988 und 2007). Dennoch argumentieren Modefirmen, außergewöhnliche und einzigartige Produkte zu schaffen, die in keiner Weise mit der Ware der Masse - und übrigens auch nicht mit der letzten kreierten Kollektion - zu vergleichen sind. So hat der Modesektor - zwischen Konservatismus und Avantgarde - eine Modalität geschaffen, die erlaubt, diese Feinheiten systematisch zu nutzen. Im Modedesign werden Feinheiten durch Schnitte, Stoffmuster, Farben und die Darstellung der Kollektion herausgearbeitet. Folglich werfen Fragen der Kreativität auch immer Fragen der nötigen Legitimität und des Verständnisses auf (Chen 2012: 636; Moeran/Christensen 2013: 3) und können als erweiterte Diskussion organisationaler Authentizitätsbemühungen geführt werden (Jones/Anand/Alvarez 2005; Suddaby u. a. 20I7).

\subsubsection{Feldmitglieder und die Umwelt}

Die Hervorbringung neuer Modekreationen ist ein kollektives Unterfangen. Es gestaltet sich als interorganisationales und internationales Geschäft eines Kollektivs, das aus hochgradig arbeitsteiligen Jobs zusammengestellt ist, deren Experten sich qua Ausbildung, Reputation und erhöhtem Erfahrungsschatz qualifizieren und dessen kreatives Produkt von Vermittlern wie Kritikern, Intermediären oder Fachmedien, die durch besondere Kenntnis bezüglich semiotischer Kodes und materieller Verwendung herausstechen, taxiert wird.

Aufgrund der Netzwerkstrukturen der Industrie hat die Designeinheit zwar größte Entscheidungsmacht. Dennoch ist der Einfluss renommierter Designer, die meist bei internationalen Großkonzernen oder angesehenen Modehäusern unter Vertrag sind, darauf nicht zu unterschätzen, was in kleineren Modefirmen als kreativ, avantgardistisch oder zeitgemäß betrachtet wird. Neben solchen in den Modemetropolen ansässigen, »großen Modeschöpfern« üben auch Akteure wie Trendforecasting-Agenturen (World Global Style Network, kurz: WGSN; Promostyl, eine der ältesten Trendagenturen; TrendTablet, ehemals Trend Union, das von der bekanntesten Trendvorhersagerin Lidewij Edelkoort gegründete Unternehmen) (vgl. Giertz-Mårtenson 2008), Modemagazine (Morean 20I2; Khaire/Hall 20I6), Modebotschafter und Models (Entwistle 2002; Godart/Mears 2009), Einkäufer (Blumer I969; Entwistle 2006) oder feldkonstituierende Ereignisse wie Modenschauen (Kühl 20I5), Modewochen (Entwistle/Rocamora 2006) oder Handelsmessen (Skov 2006; Pedersen 20I2) Einfluss auf den Rest des Sektors respektive das Feld aus und gelten deshalb nicht selten als der Ursprung von Kreations- und Innovationspotenzialen (Kawamura 2005). Schließlich konstituieren und stabilisieren reziproke Wahr- 
nehmung und Beziehungen das Feld der Mode und relativieren die marktimmanenten Unsicherheiten und Mehrdeutigkeiten.

\subsection{Fazit I: Der Creative Director im Fokus}

Dieses Kapitel hat sich mit der Selbstbeschreibung der Creative Industries und der konzeptuellen Darstellung als organisationales Feld beschäftigt. In diesem Feld wird suggeriert, dass Kreativität die Kernkompetenz schlechthin ist. Kreativität wird zum Label einer affirmativen Selbstbeschreibung eines Feldes, in dem es als vereinigender Imperativ wirkt: »Labels [...] succinctly embody what the organization means - or is hoped or intended to mean - to individuals and groups « (Ashforth/Humphrey 1997: 53). Als solches dienen organisationale Labels der Klärung und Sinnstiftung andernfalls unscharfer Sachverhalte.

Empirisch kann das organisationale Feld der Mode weiter auch von einer bestimmten Organisation aus skizziert werden, so z. B. aus der Perspektive einer Modefirma. Für die vorliegende Arbeit erscheint die Möglichkeit, eine organisationale Rolle als Feldmittelpunkt zu wählen, jedoch interessanter. Die Position des Creative Directors wird als ein Label verstanden, dessen Rolle eine besondere Funktion in der Hervorbringung von Kreativität in Modefirmen zugeschrieben wird. Als kreative Leitung übernehmen die »professional sovereigns« (DiMaggio I991: 288) Führungs- und Verwaltungsaufgaben, während sie zugleich für »alles Kreative « zuständig sind. ${ }^{55}$ So obliegt es den Creative Directors, den Inhalt, den Umfang und die Darstellung der neuen Modekollektion zu bestimmen (Godart u. a. 20I5). Ihre zentrale Unternehmensposition macht sie zu >Organisationspiloten < $-\mathrm{d}$. h. Gründer, Designer und Manager in einem (Djelic/Ainamo I999: 633). Als relevante Akteure der organisationalen Kommunikation, Interaktion und des Beziehungsgeflechts nehmen sie aktiv an der Gestaltung des organisationalen Feldes teil.

Das Feld ist der Ort der Ermächtigung und Zuschreibung von Kreativität und ihm entstammen auch Kreativitätserwartungen (Unsworth 200I). Somit ist es auch der Ursprung der Konstruktion und Attribution des Labels des »Creative Directors «, denn im Feld befinden sich nicht nur Bildungsinstitute, die Modedesigner ausbilden und über die normative Betitelung verfügen, sondern auch Kritiker und Experten, die deren Kreationen bewerten und gradieren. Entsprechend zeichnet sich das Feld der Creative Indusries durch den

35 | Wuggenig (2016: 67) thematisiert unter den Labels "Kreativ-Chef", "Kreativagentur" oder eben "Creative Director" die Zunahme und schließlich Blockierung des Terminus im Feld selber, denn der Terminus inkludiert kommerziell ausgerichtete Felder wie Werbung oder Modedesign, weshalb sich das künstlerische Feld programmatisch davon abzugrenzen versucht. 
Glauben daran aus, dass da besonders kreative Arbeit geleistet wird (Amabile u. a. I996: п166).

Das Label »Creative Director« befähigt diese Position, eine ursprüngliche Vision zu entwickeln und diese bis zur Distribution künstlerisch zu begleiten. Visionäre Ideen der Creative Directors und die kollektive Fertigung der Mode im Produktionsnetzwerk schließen sich nicht gegenseitig aus, denn

even though creative directors do not work in isolation (they oftentimes have stylists, photographers, PR professionals, assistant designers, and others who help them in their professional endeavours), they are, without question in charge of defining the vision of the collection. (Godart u. a. 2015: 201)

Obwohl dieser spezifischen Organisationsposition bedeutende, kreative Macht übertragen wird, haben auch die Akteure im Feld - sogenannte »cultural entrepreneurs « (Lounsbury/Glynn 200I; Khaire 2015) - einen Einfluss auf die Feldund Wertkonstruktion. Diese Intermediäre sind verantwortlich für »bringing about change in the meaning of the objects, beliefs in their acceptability and propriety and value, and thus in cultural conventions and norms « (Khaire 20I5: 2I5). Schließlich findet im organisationalen Feld sowohl die symbolisch-kulturelle Wertkonstruktion als auch die effektive Produktion kreativer Güter statt.

Das nächste Kapitel skizziert die Interpretationsweisen und Perspektiven von Kreativität, deren Fertigung, Bewertung und Zuschreibung aus verschiedenen Fachbereichen. Die Darstellungsweisen werden dann systematisch geordnet, um so die (organisations-)soziologische Auffassung von Kreativität zu beleuchten. 


\section{$4 \quad$ Ansätze der Kreativitätsforschung}

Gemäß der Internetenzyklopädie »Merriam Webster« ist Kreativität »the ability to make new things or think of new ideas«. Der »Duden« beschreibt das Wort als schöpferische Kraft oder kreatives Vermögen, und es wurde als solches im Jahre I973 das erste Mal im Rechtschreibwörterbuch gelistet. ${ }^{6}{ }^{6}$ Diese Beiträge verweisen auf die Unterscheidung von Kreativität als Qualität oder Fähigkeit und implizieren ein Gegenüber, das befähigt ist, diese Qualität oder Fähigkeit zu erkennen, darüber zu urteilen. In den Anfängen der Kreativitätsforschung von Mitte der I950er- bis Mitte der I970er-Jahre wurde Kreativität vor allem im Rahmen psychologischer Experimente untersucht und folglich hauptsächlich als menschliche Eigenschaft behandelt. Die fortschreitende Verwissenschaftlichung ist Resultat ungenauer psychologischer Konzepte, die divergentes oder konvergentes Verhalten nicht ausreichend erklären können. So beginnt sich die Sozialpsychologie für Kreativitätspraxen zu interessieren und ebnet in den folgenden zwei Jahrzehnten den Zugang für sozialwissenschaftliche Weiterentwicklungen. In Anschluss an die rund zehn Jahre ältere Innovationsforschung und mit wenigen Vorreitern (Howard S. Beckers Kollektivansatz von I974 und Woodman, Sawyer und Griffins ersten Versuch von I993, organisierte Kreativität zu erklären) nimmt die Soziologie nach dem Millennium das Thema in den eigenen Themenkatalog auf. 20I4 wird dann die wenig überraschende Formel des »creative turn« vorgeschlagen (Sriraman/Harris 20I4).

Was einmal als Qualität menschlicher Intelligenz galt, wird beratungsfähig und stimulierbar und wird als ehemals ausschließlich kunstsoziologisches Thema schließlich zum Interesse der Organisations- und Wirtschaftssoziologie. In der vorliegenden Diskussion des aktuellen Forschungsstandes wird darauf eingegangen, wie die Ansätze der Kreativitätsforschung in den letzten 60 Jahren floriert und sich in einem Grade diversifiziert haben, die nach einer gewissen Kategorisierung verlangen. Die Literaturübersicht findet entlang des diskursiven Verlaufs der Entwicklung des Themas vom Individuellen zum Sozialen und zum Kollektiven statt. Diese Entwicklung ist nicht als disziplinäre Differenzierung zu lesen, denn auch psychologische und verhaltenstheoreti-

36 | Zugriff am 28. Mai 2019 auf www.merriam-webster.com/dictionary/creativity. 
sche Deutungen definieren Kreativität mehr und mehr als kollektives Phänomen (Simonton 2004).

Das zentrale Argument des Kapitels jongliert mit zeitlichen, räumlichen, sozialen, materiellen und ökonomischen Elementen und versucht dabei eine >kreativitätsfreundliche< Balance zu halten. Gleichzeitig changiert es zwischen einer Vielzahl dualer Strukturen sowie Erneuerung und Tradition, Bekanntheit und Mythos, Regelgebundenheit und Freiheit, Handarbeit und Standardisierung, Kunst und Kommerz. Schließlich wird herausgearbeitet, unter welchen (organisationalen und sozialen) Voraussetzungen Kreativität möglich und wahrscheinlich wird und welche Bedingungen ex ante und in situ antizipiert bzw. ex post konstruiert werden.

\subsection{Psychologische Anfänge: Charakter und Fähigkeit}

Die theoretische und konzeptuelle Ausgangslage der Kreativitätsforschung befasst sich mit dem genieähnlichen und kreativen Individuum. Schwierigkeiten der Quantifizierung des kreativen Potenzials und dessen intersubjektive Vergleichbarkeit hinterlassen disziplinäre Mängel, die später von anderen Fachbereichen adressiert werden.

\subsubsection{Die glorreichen Tage der Kreativität und die kreative Person}

Trotz Omnipräsenz im menschlichen Leben wird Kreativität erst im Jahre I950 Objekt wissenschaftlicher Forschung (vgl. Simonton 2000). Damals wird vom Vorsitzenden der American Psychology Association, Joy Paul Guilford, auf die Geringschätzung wissenschaftlichen Interesses gegenüber Kreativität hingewiesen (I950) Der Sputnik-Schock vom 4. Oktober I957 überrascht den Westen, der sich bis dahin als Führer technologischer Entwicklungen glaubt und nun die Überlegenheit und den Kampfgeist der russischen Raumfahrtmacht einsehen muss, die den ersten künstlichen Satelliten in die Erdumlaufbahn schickt (20I2: 24). Dieses historische Ereignis begründete nicht nur das Wettrüsten des Kalten Krieges, sondern markierte auch einen Einschnitt in den Glauben an westliche Fortschrittlichkeit. Diese Geschehnisse initiieren die Kreativitätsforschung in beachtlichem Maß: Forschungsgelder werden in das neue Bildungsfeld investiert, was wiederum soziale Aufmerksamkeit und Bewusstsein dafür fördert (Bornemann 20I2; Catmull/Wallace 20I4). Sieben Jahre nach Guilfords Plädoyer an die wissenschaftliche Gemeinschaft, dem Gegenstand der Kreativität (wieder) mehr Aufmerksamkeit zu schenken, wird dieser Appell in die Tat umgesetzt, und vor dem Hintergrund und der Brisanz scheinbarer Nachlässigkeit werden kreativitäts- und innovationsfördern- 
de Maßnahmen in Bildung und Forschung eingerichtet (Mareis 20I6b). ${ }^{37}$ Das waren die »Glorious Days of Creativity« (Bornemann 20I2: 24). Von nun an wird Kreativität als Gegenstand des Intellekts in der Kognitionsforschung und als Gegenstand von Beziehungen im Bereich der Sozialpsychologie diskutiert. Die in den I96oer-Jahren entwickelten Kreativitätsmethoden, beispielsweise Brainstorming oder spieltheoretische Simulationen, verstehen sich als kybernetische und technizistische Imaginations- und Kreativitätstechniken der Nachkriegszeit in den USA, welche »die Einbildungskraft nicht einfach stimulieren, sondern diese als iterative Handlungsanweisungen vielmehr systematisch anleiten und objektivieren« sollten (Mareis 20I6a: I8) In dieser Zeit verschmelzen die empirische Bestimmung kreativer Persönlichkeitsmerkmale und die konzeptuelle Entwicklung der Kreativitätstools, welche die Entfaltung und Systematisierung anleiten (ebd.: I8).

Seit den späten I950er- und I96oer-Jahren genießt Kreativität nun mehr wissenschaftliche Beachtung, wenn auch vornehmlich psychologische. Ziele dieser Erklärungsversuche waren, neurologische und biologische Aspekte, den Zusammenhang von Intelligenz und Kreativität und ihrer Psychoökonomie, die Entwicklung, Erziehung und Ausbildung von Kreativität, »Klima und Können « in Kommunikationsräumen oder Kognitionen, die in Form von Denkweisen, Wissen und Expertise in Problemlösungsprozessen auftreten, zu erklären (Weisberg 2006; Shiu 20I4) Nach wie vor wird ein Individuum erklärt, das leistet, löst und handelt, und schließlich bleibt die Frage: »What is it that makes someone creative?« (Weisberg 2006).

Die Intelligenzforschung in der Kognitions- und Persönlichkeitspsychologie versteht Kreativität als Teil der kognitiven Intelligenz, also einer generellen Fähigkeit, bei der es darum geht, das kreative Potenzial abzurufen und auszuschöpfen (Guilford I950; Wallace/Gruber I989; Simonton I999, 2000 und 20I0). Trotz der Zentrierung kognitiver Prozesse und des individuellen Brütens über neue Ideen erfasst Guilford die Relevanz der Umwelt und betont die (soziale) Anerkennung des infrage stehenden, kreativen Verhaltens (I950: 44 f.). Der von Ellis Paul Torrance entwickelte »Torrance-Test of Creative Thinking« (I966) sollte einen Beitrag zur Versachlichung der Diskussion leisten. Der Torrance-Test war ein experimentelles Verfahren psychometrischer Erfassung, das für die Idee stand, kreative Produktivität im Sinne von Flexibilität und Flüssigkeit der Ideen messbar und vergleichbar zu machen (Amabile I982: 998; Funke 2000: 286).

37 | Eine der zentralsten Erfindungen als Reaktion auf diesen Sputnikschock war die Advanced Research Projects Agency (ARPA), die verschiedene Forschungseinrichtungen miteinander verknüpfen sollte, um weitere "technologische Überraschungen " zu vermeiden (Catmull/Wallace 2014: 10). Aus der ARPA gingen unter anderen größeren Innovationen auch die Computerrevolution und schließlich das World Wide Web hervor. 
Der Psychologe Robert J. Sternberg schloss seine Argumentation an Torrances Ideen an und versuchte, die »Natur der Kreativität« (2006 und 2007) zu erschließen. Seine und weitere psychologische Kreativitätsdefinitionen verweisen vornehmlich auf ein kreatives Individuum, dessen Persönlichkeitsmerkmale die Ursache von kreativen Fähigkeiten sind. Während konvergentes Denken das bestehende Wissen auf die Lösungsfindung eines Problems ansetzt, ist es das divergente, divergierende Denken, das zur Basis des Ideenreichtums wird und Kreativität stimulieren soll (Guilford I950). Da Kreativität als spezifische Art und Weise des kognitiven Vorgehens verstanden wird, entstammt sie dem Geist einer Person, weshalb diese Ideen nicht selten unverständlich sind und auf Widerstand stoßen (Kaufman/Sternberg 20I5). Die Ideen der »Konterformisten « betonen den Unterschied zu vorherrschenden Normen, weshalb sie als zu avantgardistisch und sozial unangemessen gelten. Da es ihnen an gesellschaftlichem Kontext fehlt, sind die Ideen oft nicht von langer Dauer. Demzufolge können Abhängigkeiten zwischen Intelligenz und Kreativität auf der Grundlage von konvergentem und divergentem Denken nicht erklärt werden (Reckwitz 2014: 226).

Spätere Ansätze der Sozialpsychologie, der Verhaltensforschung und der Soziologie bestätigen das Fehlen einer Kausalbeziehung zwischen Intelligenz und Kreativität: Kognitiv-logisches Denken ist nicht selten konform mit gesellschaftlichen Erwartungen und Regeln und würde insofern relativ weniger mit potenzieller Kreativität korrelieren. Vielmehr führt der Mittelweg, das Beachten und Spielen mit sozialen Vorgaben, zu verständlichen und als kreativ bewerteten Ideen, was wiederum zu ihrem gesellschaftlichen Erfolg beiträgt. Dennoch bleibt die Generierung von Ideenvielfalt - oft auf divergentem Denken basierend - ein zentrales Merkmal, das mit Kreativität in Verbindung gebracht wird:

Creativity is the ability to come up with ideas or artefacts that are new, surprising and valuable $[\mathrm{A} / \mathrm{N}]$. , Ideas , here include concepts, poems, musical compositions, scientific theories, cookery recipes, choreography, jokes - and so on. 'Artefactsı include paintings, sculptures, steam engines, vacuum cleaners, pottery, origami, penny whistles - and many other things you can name. (Boden 2005: 1)

Gemäß Boden ist es die Fähigkeit, mit neuen, überraschenden und wertvollen Ideen oder Artefakten zu überzeugen, die ein kreatives Wesen ausmacht.

Weiterhin stehen psychologische Konzepte wie Charakteristika und Fähigkeiten im Zentrum der Kreativitätsdefinitionen. In diesem Ansatz gibt es aber nichts, das als »kreative Praxis« ausgewiesen werden könnte, und es fehlt jedwede Vorstellung davon, wie Kreativität als intersubjektive Leitidee begrifflich gefasst werden könnte. Zunehmend fällt aber auch der Psychologie auf, dass nicht nur Individuen Kreativität zugeschrieben wird, sondern oft nicht kreative 
Personen in Gruppen Neues schaffen, das sie alleine nicht hätten erreichen können (Simonton 2004; Sacramento/Dawson/West 2008). So wird Kreativität immer mehr als komplexes Phänomen verstanden, dessen Untersuchung über die methodischen Mittel der Psychologie hinausgeht (Simonton 2000: 155).

\subsubsection{Kreativität als Verhalten oder Attribut}

Während Guilfords Prinzipien im engeren Sinne kognitions- und entwicklungspsychologisch begründet waren, setzte mit dem Interesse der Sozialpsychologie und der Managementtheorien eine »Versozialwissenschaftlichung « der Kreativität ein. Diese perspektivieren Kreativität als Gegenstand zwischenmenschlicher Beziehungen und problematisieren Themen wie Optimierungsdrang, Talent versus Ausbildung, intellektuelles Spiel und Motivation sowie Engagement und Leistung als grundlegende Kriterien (Cummings I965; Amabile 1996; Amabile u. a. 1996; Amabile 1998; Kurtzberg/Amabile 2010; Gong/Kim/Lee 20I3; Lee/Walsh/Wang 20I5). Die kognitivistische Kreativitätspsychologie versucht, »eine >wahre< Kreativität von einer nur scheinbaren abzugrenzen, wobei Letztere entweder ein Zuviel an Sozialorientierung enthält (der modische Konterformist) oder aber ein Zuwenig (die Absurdität des Verrückten oder Kuriosen)«s schreibt der Praxissoziologie Andreas Reckwitz in seiner »Erfindung der Kreativität« (20I4: 227).

Die Sozialpsychologie versteht unter Kreativität, was eine Gemeinschaft als kreativ bewertet bzw. was in Übereinstimmung mit der Darstellung eines bestimmten kreativen Prototyps steht (Amabile I982 und I983; Csíkszentmihályi I988; Elsbach/Kramer 2003). Dabei wird Kreativität nicht als Charaktereigenschaft oder Fähigkeit beschrieben, sondern ist »[...] a behavior resulting from particular constellations of personal characteristics, cognitive abilities, and social environments « (Amabile I983: 358). Zunehmend lässt sich eine Verschiebung der dominanten Sichtweise von kognitivistischen, persönlichkeitszentrierten Betrachtungsweisen hin zu einer Interpretation feststellen, welche die soziale Einbettung und Bedingtheit hervorheben. Dadurch erlangte der »Attributionsfehler« mehr Aufmerksamkeit:

This leads to the fundamental attribution error, which occurs when one person is doing something, and someone else is observing. The latter not surprisingly is called "an observer, "but the former is assumed to be involved in some act or action and is thus called (less intuitively) the "actor." In general, an actor's attention is directed to the act, whereas an observer's attention will tend to be on the actor him- or herself. [...] So when asked to explain the action, the actor considers what he or she saw - namely, the immediate environment, the setting, the context. The observer, on the other hand, tends to explain the very same action in terms of the actor's ability or personality. (Runco 2007: 159) 
Die Zuschreibung von Kreativität ist umstritten, weil vermeintliche und sechte< Hinweise auf einen kreativen Charakter (von Personen, Produkten oder Prozessen) ungenau feststellbar und selten trennscharf sind. Nun werden die situativen Gegebenheiten für die kreative Leistung verantwortlich gemacht; jedoch nur dann, wenn persönliche Eigenschaften besonders auffällig sind, wird auf diese als Ursache herausragender Kreativität verwiesen (Amabile I998). Scheinbar wird besonders stark an das kreative Talent geglaubt, wenn konträre Charaktereigenschaften auffällig sind, wie beispielsweise der taube Komponist, der gelähmte Astronaut, der Unternehmer, der es vom Tellerwäscher zum Millionär gebracht hat, oder der psychisch erkrankte Künstler: »Such creators are considered geniuses not only because of their creations but also because of their handicaps« (Kasof I995: 3I7 zit. in Elsbach/Kramer 2003: 292). Die Idee der Zuschreibung von Kreativität wird nicht per se verworfen, sondern in einem stärker soziologischen Kontext behandelt, wobei ein befähigtes Expertentum oder Publikum die Bewertung vornimmt und sozial zuschreibt.

Die Verschiebung des Forschungsmittelpunkts vom Individuum zur Umwelt lässt nunmehr kein induktives Erschließungsverfahren mehr zu, sondern normalisiert Kreativität im Alltag als >kreativer Habitus $<$, der durch eine Vielzahl psychologischer Praxeologien, »ein Ensemble von inkorporierten Schemata und Strategien, die das Subjekt permanent und scheinbar ganz natürlich zur lustvollen Wahrnehmung und Hervorbringung des Neuen [...] befähigen« (Reckwitz 20I4: 228), begünstigt wird. Konzeptuelle Studien haben den Einfluss situativer Faktoren auf Kreativität (Cummings I965; Woodman u. a. I993) thematisiert, während die empirische Forschung den Fokus auf Faktoren am Arbeitsplatz gerichtet hat (Amabile u. a. I996; Andriopoulos 200I; Perry-Smith 2006; Watson 2007; Unsworth/Clegg 20I0; Jia u. a. 2013). Dennoch kann diese fortwährend stark psychologisierende Kreativitätsdarstellung kaum als Dialog zwischen soziokulturellem Kontext und kognitiven Denkprozessen verstanden werden. Ebenso wird kaum darauf eingegangen, dass Kreativität das Resultat einer Vielzahl von Prozessen und Akteuren ist (Tschmuck 20I2: 269).

\subsubsection{Die Bedeutung sozialer Gutheißung}

In der Schule der »positiven Psychologie« argumentiert der Psychologe und Kulturanthropologe Mihaly Csíkszentmihályi (I988; 2007), dass kreativ ist, »who brings about some change in the domain, a change that the field will consider to be creative « (I988: 325). Csíkszentmihályi gehörte einer professionellen Bewegung von Anthropologen an, denen man das Verdienst zuschreibt, die ersten gewesen zu sein, die Fragen der sozialen Entstehung und Bewertung kreativer Leistung zu einem Sachverhalt von Wissen machten, und die versuchten, die phänomenologische und relationale Dimension der Thematik den experimentellen Auseinandersetzungen vorzuziehen und somit Kreativität 
als sozial konstruiertes Konzept definieren. Er entwickelt eine Art »Lustprinzip« der Kreativität, das ein Flow-Erlebnis auslösen und gewöhnliche Aktivitäten in herausfordernde Situationen transformieren kann (Csíkszentmihályi I996). Gemäß Csíkszentmihályi ist das Auftreten von Kreativität durch drei Elemente bedingt: das Individuum, die Domäne und das Feld (ebd. 2007: 314). Diese Dreiheit verschiebt den Fokus vom einzelnen Schöpfergeist über die Produktion des Objekts hin zur kritischen Bewertungsinstanz. Diese Konzeptualisierung findet Anschluss in multidimensionalen Definitionen, die einen Kreateur (welcher durchaus auch eine Gruppe sein kann), ein Objekt und ein Publikum (oder die Konsumenten oder Rezipienten) voraussetzen (Barrett I998; Hargadon/Bechky 2006; Carlsen u. a. 20I3; Reckwitz 20I4 und 20I6; Sonenshein 20I4).

Durch diese konzeptuelle Öffnung wurden verschiedenen disziplinären Feldern Anschlussmöglichkeiten geboten, was der Kreativitätsforschung weiteren Aufschwung verlieh. Gerade die praxisorientierte Kreativitätsdefinition leistet vielerlei Beiträge für Forschung und die Praxis (Drazin/Bird Schoonhoven 1996; Oldham/Cummings I996). Insbesondere lässt sich nun zwischen einer psychologischen Kontextualisierung, bei der das Individuum als kreativer Akteur im Mittelpunkt steht, und einer eher sozialwissenschaftlichen, die den Akteur in ein kreatives (genauer: kreativitätsförderndes respektive -hinderndes) Umfeld einbettet, unterscheiden. Hierbei scheint die Domäne als zentrales Konzept zu dienen, die sowohl den sozialen wie auch den kognitiv-intellektuellen Kontext für die Einbettung von Individuum und Idee darstellt (Amabile I983; Csíkszentmihályi I988). Im Zentrum dieser Konzeptionen stehen die Erfahrung und Analyse kreativitätssteigernder (Selbst-)Techniken.

Theresa M. Amabile begreift Kreativität als Resultat von Konsens in einer Domäne:

A product or response is creative to the extent that appropriate observers independently agree it is creative. Appropriate observers are those familiar with the domain in which the product was created or the response articulated. Thus, creativity can be regarded as the quality of products or responses judged to be creative by appropriate observers, and it can also be regarded as the process by which something so judged is produced. (Amabile 1982: 1001; Hervorh. der Verf.)

Diese Begriffsdefinition basiert auf einer wahrnehmungsspezifischen und resultat-orientierten Erklärung von Kreativität, weil eine spezifische Qualität von Beobachtern wahrgenommen wird, die mit einer positiven Bewertung darauf reagieren und Kreativität zuschreiben. Sie beruht auf drei Komponenten: erstens aus domänen-relevanten, spezifischen Fähigkeiten, wie beispielsweise das Beherrschen einer bestimmten Technik, die zumeist nicht in angeborenem Talent, sondern Sozialisierung und Ausbildung gründen; zweitens kre- 
ativitätsrelevante Fähigkeiten, die durch bestimmte kognitive wie auch heuristische Arbeitsweisen begünstigt und somit trainiert werden können; und drittens die Arbeitsmotivation, die motivationale Faktoren wie Einstellungen und Wahrnehmung der intrinsischen und extrinsischen Beschränkungen umfasst (Amabile i983). Soziologisch ist dabei relevant, dass die Zuschreibung von Kreativität nicht nur maßgebend auf Konsens basiert (was als kreativ wahrgenommen wird, ist gesellschaftlich festgelegt). Vielmehr unterstreicht diese Definition zwei zentrale Punkte: Erstens besteht eine Korrelation von Wissen und Beherrschen bzw. Ausnutzen des intellektuellen Spielraums (der sich zumeist bei Experten eines Gebiets und nicht deren Novizen zeigt (Shalley/Gilson/Blum 2000: 2I6 f.), und zweitens handelt die Organisation vorgreifend, als hätte die Kreativitätszuschreibung schon stattgefunden (Koch u. a. 20I8). Mit entsprechenden Darstellungsmethoden können nun nicht nur Personen, Industrien oder Ökonomien, sondern auch Städte oder Klassen als kreativ bezeichnet werden (Wuggenig 20I6: 59). Selbst die Darstellung von Kreativität kann kreativ sein, und diese Selbstdarstellung informiert über die eigene Feldzugehörigkeit und Verortung.

In späteren Arbeiten taxiert Amabile (I995, I996 und I998) das Klima für kreatives Arbeiten, in dem sie soziale und umweltbedingte Einflussfaktoren auf die Kreativitätshervorbringung untersucht und daraus ein Modell entwickelt, das Anreize und Hinderungsgründe von Kreativität fassbar macht. Die Beurteilungskriterien beinhalten $u$. a. die kreativitätssteigernden Maßnahmen wie Ermunterung und Betreuung bei der Arbeit, Freiheit und ausreichende Ressourcen. Als kreativitätshemmende Faktoren werden der Druck durch das Arbeitspensum oder organisationale Hinderungsgründe genannt (Amabile u. a. I996). In diesem Sinne besitzt das Management die Rolle der Kreativitätsförderung oder -hinderung und hat die Möglichkeit, das kreative Potenzial zu beeinflussen. Kreativität kann zwar gefördert oder gehemmt werden, und dabei liegt der wissenschaftliche Fokus noch immer auf dem Individuum, jedoch werden die Einbettung, die Gruppe, das Arbeitsumfeld, der weitere institutionelle Kontext oder die Gesellschaft zu einschlägigen Faktoren der Kreativitätsbewertung. Die Sozialität der Kreativität ist bedeutend, denn ihr entstammt Akzeptanz oder Ablehnung.

\subsection{Sozialtheoretische Weiterentwicklungen}

In den anschließenden Jahrzehnten richtet sich die Kreativitätsforschung vornehmlich an nicht psychologischen, intersubjektiven Argumenten aus, »weg vom Mythos der individualistischen Qualität der Kreativität hin auf ihre grundlegende Sozialität« (Wuggenig 20I6: 72 f.). Trotz weniger soziologischer 
Referenzen fristet das Thema weiterhin ein Nischendasein in der Wirtschaftsund Organisationssoziologie.

Ungeachtet der psychologischen Dominanz hat sich die sozialpsychologische Kreativitätsthematik als anschlussfähig erwiesen. Dennoch dienten stark psychologisierende Konzeptionen bis in die Gegenwart hinein vornehmlich als Kontrastfolie, von der man sich programmatisch abzugrenzen versuchte. Die relationale Betonung schöpferischer Kontakte hat demgegenüber mächtig Anschluss gefunden (Hargadon/Bechky 2006; Taylor/Greve 2006; Carlsen u. a. 20I3; Sonenshein 20I4; Lee u. a. 20I5). Jüngere Diskussionen nehmen die daraus entsprungene soziale und kollektive Kreativitätsdimension zum Ausgangspunkt. Dabei nimmt die Soziologie eine besondere Stellung ein, da sie aufgrund ihrer vielschichtigen Erklärungsleistung auch interdisziplinäre Themengebiete anspricht.

\subsubsection{Kreativität als Merkmal kollektiver Sensibilisierung}

Professionalisierungstendenzen und gemeinsame Berufsbilder bringen ein >geschultes Auge hervor, wodurch eine Immersion mit der Kultur- und Kreativwelt entsteht, die in einer kongruenten Weltanschauung der Individuen mündet (DiMaggio I99I). Festgestellt wird, dass diese mehrstufige Applikation verschiedener Filterebenen (individuell, professionell und industriell) eine häufige Erscheinung in der Kulturproduktion ist (Hirsch I972: 640). Die soziologische Kreativitätsforschung präferiert eine Kultur- und Prozessperspektive gegenüber einer stärkeren - wie sie in der Innovationsforschung prominent ist - Strukturorientiertheit. Weil die meisten Organisationen auch kreativ sein wollen (oder sollen), lässt sich die Hervorbringung von Kreativität als ein organisierter, kontinuierlicher Prozess begreifen (Fortwengel u. a. 20I7).

Die Modeindustrie ist ein Beispiel dafür, wie die Professionalisierung von Akteuren entscheidungsrelevante Strukturen einer Kultur entwickelt, die zur institutionellen Angleichung führen und dadurch ihr Denken und Handeln bestimmen. Blumer beschreibt in seiner soziologischen Analyse der Modeindustrie, wie sich das ästhetische Bewusstsein und schließlich die getätigte Auswahl der Einkäufer angleicht. Diese »Geschmackskonvergenz« wird als Resultat durchdringender Einbettung in eine gemeinschaftliche Welt erklärt, die dadurch gekennzeichnet ist, dass Einkäufer verbreitete Sensibilitäten und ähnliche Wertschätzungen ausbilden (I969: 279).

Csíkszentmihályi betrachtet (geteilte) Auswahl- und Bewertungsverfahren als zentrales Moment der Zeithaftigkeit neuer Ideen und betont dabei Methoden des Vergleichs, der Bewertung und der Auffassung und Auslegung:

Of course, like most people, I have been exposed to many objects and ideas that we call creative. Some of these might have been arresting, or interesting, or impressive, 
but I cannot say that I ever thought of them as icreative،. Their creativity is something that I came to accept later, if at all, after comparing the object or idea with others of its kind, but mostly because I had been told by experts that these things were creative. The only way to establish whether or not something is creative is through comparison, evaluation, and interpretation. (1988: 326 ff.)

Kreativität ist somit doppelt relational: Erstens als Bewertung und zweitens als Vergleich wird sie auf Anderes bezogen. Dabei verhält sich Kreativität ziemlich ähnlich wie andere vermittelte Konstrukte: Je bekannter und professioneller die Expertise der Bewertungsinstanz, desto mehr Legitimität erhält das Urteil (Meyer/Rowan I977; Suchman I995). Ebendies gilt auch für Kreativität: Etwas, das nicht legitim oder akzeptiert ist, kann nicht kreativ sein (Cattani/Ferriani 2008: 826). Demnach ist Kreativität in gleicher Weise wie Legitimität sozial bedingt, denn das Kreative soll mit den Erwartungen der sozialen Bewertungsinstanz übereinstimmen. Kreativität ist, wie Legitimität auch, von einem kollektiven Publikum abhängig, jedoch unabhängig von den einzelnen Beobachtern (Suchman I995: 574). Um mehr Verständnis und Zustimmung aus dem allgemeinen Publikum zu erhalten, müssen die Dimensionen Bewertung und Vergleich um eine dritte Dimension ergänzt werden: soziale Nachvollziehbarkeit. Totale Neuheit führt zu Unverständnis im Publikum. Demgegenüber passen sich (Er-)Neuerungen an vorherrschende Bedingungen an und wirken dadurch legitimierend (Hargadon/Douglas 200I). Soziale Nachvollziehbarkeit bedeutet, dass Neuerungen in eine Vielzahl bestehender Traditionen und Normen hineingeboren werden, weshalb wir sie nur wahrnehmen können, »unless it somehow relates to what we already know « (Czarniawska/Joerges i996: 27). In diesem Kontext stellt Kreativität ein gesellschaftliches Merkmal dar.

\subsubsection{Kreativität als Gesellschaftsmerkmal}

Die omnipräsente Kreativitätserwartung findet sich auf unterschiedliche Weise in der Literatur wieder: Einmal ist von »Kreativitätseuphorie« die Rede (Wuggenig 20I6: 42), dann werden das gesellschaftliche »Kreativitätsdispositiv« und der »kreative Imperativ« erklärt (Reckwitz 20I4) oder die Metapher der »Raupe Nimmersatt« verwendet (Hasse u. a. 20I9 forthcoming). Die Akteure des Kunstfeldes reagieren gegenwärtig mit einer reflexartigen Abweisung des Begriffs der Kreativität (van den Berg 2009), weshalb die Debatte auch als »Kritik der Kreativität« rezensiert wird (Osborne 2003; Raunig/Wuggenig 2016).

Die Kreativitätserwartung wird von einer allgemeinen Wahrnehmungstransformation vorangetrieben, die maßgeblich von der Beratungsliteratur betrieben wird und sich für kreative Selbsttechniken und soziale Anerkennung der kreativen Leistung einsetzt, weshalb ein positives Gefühl des Krea- 
tiv-Sein-Wollens entsteht (Reckwitz 20I4: 228 ff.). Das Neue wird durch das Alte bedingt, ihm gegenüber bevorzugt, kann aber nie als Neues einfach objektiv vorhanden sein. Diese Alt/Neu-Unterscheidung führt zu normativ positiver Bewertung des Anderen (gegenüber dem Gleichen), des Abweichenden (gegenüber dem normativ Erwartbaren und Normalen). Es handelt sich um eine Bewertung, »die allerdings von sozialen Kriterien abhängt, die besagen, welches Neue wertvoll ist und welches nicht« (Reckwitz 20I4: 44). In der von Kreativität dominierten Gesellschaft wird dieses Verhältnis zu Neuerungen als grundlegende Ursache zur Entwicklung des Kreativitätsdispositivs und weiter auch der Creative Industries gesehen.

Was für Reckwitz das »Kreativitätsdispositiv« ist, wird vom Soziologen und Ökonomen Richard Florida als »kreative Klasse« (2006) und von Charles Landry als »kreative Stadt« (2004) beschrieben. Diese Konzepte bauen auf der Wissensgesellschaft auf, in der Kreativität die grundlegende Ressource ist, die sowohl zur individuellen und sozialen Entwicklung und zu Problemlösungen beiträgt als auch als Standortvorteil urbaner Räume wirkt. Deshalb ist für die wissensbasierte Gesellschaft keine andere Fähigkeit gleichermaßen wichtig.

\subsubsection{Kreativität als Problemlösungsmodus}

Fortan entwickelt sich Kreativität als Produkt interaktiver Zusammenarbeit in zwei Stoßrichtungen. Aus der Interaktion entsteht eine »supra-individual creativity«, ein Problemlösungsprozess, bei dem Synergien genutzt werden und der in einem Moment »when individuals come together to find, redefine, and solve problems that no one, working alone, could have done as easily, if at all« zum Tragen kommt (Hargadon/Bechky 2006: 486 f.). Es wird argumentiert, dass Kreativität als problem-basierte, heuristische Vorgehensweise das Ergebnis von Interaktionen ist (interaktionistische Begriffsdefinition), aus einer Vielzahl von Variablen hervorgeht und sich so je kontextspezifisch konstituiert. Im Problemlösungsmodus treffen in Gruppen verschiedene Wissensvorräte und Erfahrungswelten aufeinander, kollidieren und konvergieren. Eine Vielzahl von Studien argumentiert, dass Spannungen innerhalb heterogener Gruppen Kreationspotenziale freisetzen und Leistungen steigern (Oldham/ Cummings I996; Madjar u. a. 2002; Gilson u. a. 2005; Perry-Smith 2006; Taylor/Greve 2006; Harvey/Kou 2013).

Weiter verändern Kreativität und Innovation als Bestandteil von Wandel die Sicht- und Deutungsweisen sozialer Akteure, wodurch Entscheidungsoptionen beeinflusst und individuelles Handeln erklärt werden können (Weick/ Sutcliffe/Obstfeld 2005; Hargadon/Bechky 2006; Obstfeld 20I2). Demnach muss die Bedeutung neuer wie auch alter Ideen stets neu ausgehandelt werden. Drazin, Glynn und Kazanjian definieren Kreativität folglich nicht als Aggregat individueller Kreativitätsperformances auf Gruppenebene, sondern als 
»the process of engagement $[\mathrm{A} / \mathrm{N}]$ in creative acts, regardless of whether the resultant outcomes are novel, useful, or creative « (1999: 287). Kreativität findet folglich dann statt, wenn sich eine Gruppe in einen Prozess kreativer Arbeit vertieft, unabhängig von dessen Resultat oder Erfolg.

Mithilfe von trainiertem Wissen, Fähigkeiten und Fertigkeiten wie auch den entsprechenden Hilfsmitteln und Werkzeugen wird auch das Außenvorlassen professioneller Expertise als kreativitätsfördernd illustriert, denn Tüfteln stimuliert das out-of-the-box-Denken. Schumacher und Glaser (2008) beschreiben die »Mythologisierung der Architekturausbildung « mit einer besonders fachfremden Kreativitätspraxis, die mit professionellen Arbeitsweisen bricht und vielmehr alltagsweltliche Praxisabfolgen zeigt. Durch diese Laienstrategie wird beabsichtigt, kreative Vorgehensweisen in die eingespielte und routinierte Praxisabfolge einfließen zu lassen und verschiedenartige Materialen in neuer Form, z. B. einer Collage, zu kombinieren. Dadurch wird das Befremdliche, Nichtprofessionelle zur Möglichkeit des kreativen Ausdrucks. Schumacher und Glaser befinden Bricolage als »nicht-professionelle kreative Praxis, [...] die improvisierte Rekombination und Assoziation des bereits vorgefundenen Materials« (2008: 24 f.). Mangelndes Wissen, fehlende Ausrüstung, Tüfteln und Improvisieren schaffen die Voraussetzung dafür, dass Neues entstehen kann. Kreativ ist dann das Spiel mit vertrauten Alltagspraktiken und Ressourcen, das lediglich eines anderen Blicks bedarf. Ein Beispiel dafür ist die sozialwissenschaftliche Beratung, deren fremde Perspektive zu kreativen und innovativen Ideen in Unternehmen führen kann (Lau/Brok-Kristensen in Pratt u. a. 2015: 292 ff.).

Kreativitätstheorie befasst sich häufig mit dem Ansatz, dass Kreativität zum Lösen bestehender Probleme dient. In der Literatur wird dieser Typ von Kreativität als »small-c creativity« bezeichnet (Simonton 2000; Gilson 20I5). Kreativitätsdefinitionen lassen sich entlang eines Kontinuums verorten und die »small-c creativity« umfasst Problemlösungsprozesse in Alltagshandlungen. Dieser Sichtweise unterliegt eine alltägliche und industrie- bzw. professionsunspezifische Deutung von Kreativität: Kreativ ist das handwerkliche Tüfteln, das Ausprobieren und Umgestalten, das dabei hilft, Probleme effektiv zu lösen oder abweichendes Verhalten zu kaschieren. In diesem Sinne ist diese Kreativitätsdefinition kaum sichtbar und wird selten hervorgehoben, denn sie soll aufgrund der Lösungsorientierung - das Resultat, nicht der Weg ist das Ziel - nicht sichtbar sein. Kreativität ist das Mittel (die Problemlösung) zum Zweck (der intendierten Lösung), weshalb die erzielten Erfolge selten als kreativ ausgewiesen werden. Dieses Konzept der Alltagskreativität ist eine allgemeine Form der Kreativität und in allen Industrien, Organisationen und Professionen zu finden.

Im Gegensatz zum alltäglichen Tüfteln treibt die Creative Industries die Entwicklung, Produktion und Distribution gesellschafts- oder kulturrelevan- 
ter Güter (Produkte und Dienstleistungen) an. Es handelt sich um die sogenannte Big-C Creativity ((Simonton 2000; Gilson 20I5). Obwohl das Problemlösen zum alltäglichen Handeln aller Organisationen gehört, unterscheiden sich diese spezifischen Industrien, Organisationen oder Professionen von anderen, weil geglaubt und erwartet wird, dass sie kreativ sind.

Unsworths (200I: 29I f.) Matrix der kreativen Problemlösung enthält vier Kreativitätstypen, die entweder als offene oder geschlossene sowie entweder intern oder extern motivierte Probleme kategorisiert werden. Gemäß dieser Matrix sind Prozesse und Produkte der Creative Industries Beispiele des Typs der erwarteten Kreativität, denn sie sind die erforderten Lösungen der definierten Probleme, deren Methode der Umsetzung jedoch unklar ist. In den Creative Industries wird der Prozess des Kreierens visuell und/oder textuell vermittelt. Die Neuerung und deren Innovativität werden z. B. über Werbung und Marketing kommunikativ ausgewiesen. Während solche auf Kreativität bezogenen Sprachspiele eine Eigenheit kreativer und kultureller Organisationsformen darstellen, sind Tüfteleien keine Seltenheit - nicht bei Individuen und noch weniger bei Organisationen.

Das Problemlösen nach Algorithmen oder Heuristiken thematisiert Kreativität folglich als Kontinuum und nicht dichotomen Sachverhalt. Am einen Ende befindet sich das verlässliche, regelhafte Abarbeiten von Algorithmen, am anderen Ende das Anwenden regelmissachtender Heuristiken und das Erkunden komplett neuer kognitiver Pfade (Amabile I983: 37I). Problemlösen nach Algorithmen versucht, die Ordnung aufrechtzuerhalten (»maintain order in the face of change «), während das Problemlösen nach Heuristiken beabsichtigt, die Ordnung zu unterbrechen (»maintain change in the face of order«):

Our understanding of collective creativity differs from collective mind, however, in that our focus is on the processes that generate creative disruptions from the established order as opposed to maintaining order in the face of disruption. [...] in these organizations the ends of the enterprise are clear - to maintain order in the face of change. In contrast, in settings requiring creativity, as the goal of the process is to create new solutions - to maintain change in the face of order. (Hargadon/Bechky 2006: 495)

Der erste Problemlösungsmodus bezieht sich auf die Aufrechterhaltung von Ordnung im Angesicht des Wandels - Probleme so zu lösen, dass sie zum erwarteten Ziel führen (small-c creativity). Der zweite Problemlösungsmodus intendiert jedoch die Beständigkeit und Ordnung durch neue Lösungswege zu unterbrechen. Weil die Ordnung scheinbar vorherrscht, werden innovative und kreative Prozesse sichtbar gemacht. Insbesondere die Creative Industries sind Experten der Sichtbarmachung, wodurch sie sich von anderen Industrien abzuheben versuchen. Kreativität wird zum Selbstzweck. 
Die Kreativitätsforschung schließt damit an Beiträge der Organisationsforschung, z. B. das »exploration-exploitation«-Dilemma (March I99I; Tuschman/Benner 2003), oder dem in der Innovationsforschung entwickelten Ansatz der inkrementellen/problemzentrierten und radikalen/ lösungsorientierten Kreativität an (Gilson/Madjar 20II)..$^{8}$

38 | Gilson und Madjar (2011: 22) definieren radikale respektive inkrementelle Kreativität wie folgt: "Highly radical ideas should suggest new and set-breaking frameworks or processes. [...] In contrast, incremental ideas are those that imply changes in frameworks and approaches and modifications to the existing practices and products." Radikale Kreativität wird maßgebend von intrinsischen Motiven (Auseinandersetzung, Experimentieren oder Hartnäckigkeit) der Individuen geprägt, während inkrementelle Kreativität von extrinsischen Motiven (Belohnung, externer Stimuli) evoziert wird. 


\section{$5 \quad$ Kreativität als Gegenstand der Organisationsforschung}

Nach der Einführung der psychologischen Sichtweise auf Kreativität und der Vertiefung in vereinzelte Aspekte der sozialwissenschaftlichen Agenda werden in diesem Kapitel organisationstheoretische bzw. managementbezogene Erklärungsansätze der Kreativitätsforschung aufgegriffen. Dazu wird der in der Kreativitätsforschung prominente, netzwerktheoretische Ansatz aufgenommen. Netzkontakte sind in den Creative Industries und im Spezifischen in der Modebranche vor allem für die Produktion wichtig. Als Orte des Vertrauens und der Innovation tragen sie zwar auch zur Entwicklung, vor allem aber zur Umsetzung neuer, kreativer Ideen bei. Obwohl die Anlage des vorliegenden Forschungsdesigns kaum Rückschlüsse auf die Einbettung der Modefirmen in Netzwerke zulässt, wird an dieser Stelle trotzdem Bezug genommen darauf, denn im Rahmen der Projektkooperation sind temporäre Kontakte $-d$. h. freie Mitarbeiter - eine wesentliche Bedingung der Kreativitätshervorbringung. Zudem wird für die Betrachtung aus einer organisationalen Managementperspektive an Techniken und Praktiken zur Generierung von kreativen Ideen angeknüpft. Die organisationale Sichtweise hebt folglich die innerbetrieblichen Fördermaßnahmen hervor.

Richard Woodman, John Sawyer und Ricky Griffin stellten 1993 eine Definition organisationaler Kreativität vor, welche an die Vorläufer verhaltenspsychologischer Forschungsrichtungen anknüpft und sie mit der organisationstheoretischen Innovationsforschung verbindet. Diese interaktionistische Begriffsdefinition legt organisationale Kreativität als »the creation of a valuable, useful new product, service, idea, procedure, or process by individuals working together in a complex social system « des erstmaligen Auftretens einer Sache oder neuen Wissens fest (Woodman,/Sawyer/Griffin 1993: 293). Kreativität ist Teil von organisationalen Innovationen, die wiederum Teil organisationalen Wandels sind. Sie ist das Resultat von Kooperationen und kollektiven Prozessen. Wandel in Organisationen führt nicht zwingend zu Innovationen, aber Innovationen können sich auch in der »Minimalform« als Anpassungen oder Änderungen zeigen (ebd.: 293). Diese Weiterentwicklung richtet den Fo- 
kus erneut auf die soziale Koordinierung und Gestaltung von Neuerungen. Mit diesem Aufsatz tragen Woodman u. a. zur allgemeinen Salonfähigkeit des Gegenstands in der Organisations- und Managementforschung bei. Diese kollektive, organisationale Perspektive hat eine Vielzahl empirischer Studien hervorgebracht (Hargadon/Bechky 2006; Mora 2006; Krämer 20I4).

Zunehmend kann als Folge der strukturellen Prominenz der organisationssoziologischen Diskussion eine Prozessperspektive verzeichnet werden (Fortwengel/Schüssler/Sydow 20I6; Garud u. a. 2or6b). Diese versteht Neuerungen als emergenten Gegenstand, der als kontinuierlicher Prozess durch die Zeit-, Raum- und Relationslinse betrachtet wird und jenseits von Objekten Wirkung entfaltet (Garud u. a. 2oI6a). Außerdem bearbeitet die prozessuale Perspektive nicht nur auf Technologien und Produkte bezogene Themen der Innovationsund Kreativitätsforschung, sondern auch Angelegenheiten sozialer Innovationen, zu denen auch organisatorische Neuerungen zählen (Garud u. a. 20I3).

Im ersten Kapitelteil wird auf zwischenorganisationale Netze eingegangen, dem ein zweiter Teil zum innerbetrieblichen Management der Kreativität - wie beispielsweise Rekombinationspraktiken - folgt. Die Schilderungen verschieben die ursprünglichen »flachen« Kreativitätskonzepte hin zu einem mehrdimensionalen Begriff, der sich in der Lage sieht, der Dynamik und dem Wandel der sozialen Wirklichkeit - und somit der Natur kreativer Prozesse - gerecht zu werden. Kreativität entsteht sowohl aus einzelnen Organisationsformen als auch zwischen Organisationen.

\subsection{Kreativität zWischen Organisationen}

Gleich wie in der Innovationsforschung, so hat man versucht, Einsichten der Netzwerkforschung in der Kreativitätsforschung zu nutzen. Netzwerke können als analytisches Werkzeug der Erhebung sozialer Beziehungen oder als multilaterale Koordinationsform definiert werden (Powell/Smith-Doerr I994). So hat schon Stinchcombe (I959) in seiner vergleichenden Studie Netzwerke als die typische Koordinationsform herausgearbeitet, in welchem Neuerungen durch Projektorganisationen hervorgebracht werden. Netzwerke sind nicht nur für die Rekrutierung von Personal oder für den Informationsfluss bedeutsam, sondern über sie werden auch gute Ideen verbreitet (Burt 2004).

Das vorliegende Kapitel interessiert sich für Netzwerke als »sozialer Leim« der Ökonomie der Kulturproduktion, der die Abhängigkeiten und Verbindungen unter den Akteuren des organisationalen Feldes über formale und informelle Kommunikationskanäle sichtbar macht und sich in Praktiken der Vertragsvergabe, kooperativer Fertigungswirtschaft und zwischenbetrieblichen Bündnissen offenbart (Powell/Smith-Doerr 1994: 369). Entsprechend werden Beziehungsarten, Fragen der Kohäsion und die Positionierung von Akteuren 
als Einflussfaktoren von Kreativität identifiziert (Perry-Smith 2006; Taylor/ Greve 2006; De Vaan/Stark/Vedres 20I5). Feldstudien konnten aufzeigen, dass gute Ideen maßgeblich auf nicht redundante und über Dritte vermittelte Informationen zurückgehen bzw. auf die Zusammensetzung von heterogenen Informationsquellen (Uzzi/Spiro 2005; Cattani/Ferriani 2008; Perry-Smith/ Mannucci 20I6). Die im Netzwerk verbreitete kognitive Diversität stimuliert divergentes Denken, erweitert das Repertoire der Rekombinationsmöglichkeiten und übt einen positiven Einfluss auf die kreative Leistung und deren Erfolg aus (Garud u. a. 20I3: 779). Zugang zu neuem Wissen erhöht dabei die Wahrscheinlichkeit, auf andersartige Ideen zu kommen, wobei der Neuheitsgehalt der Information ebenso wichtig ist wie deren Inhalt (Hargadon/Sutton 1997; De Vaan u. a. 20I5; Lampel 2008). Hieran anknüpfende Forschungsbeiträge behandeln die Kreativindustrie selbst als soziales Netzwerk, das neue Märkte schafft und alternative Wahlmöglichkeiten bietet (Potts u. a. 2008).

\subsubsection{Produktionsnetzwerke}

Mit der Grundlage Beckers »Art Worlds« hat die Netzwerkforschung in der Kreativitätsforschung konzeptuell wie auch empirisch stark Anschluss gefunden. Erstens bietet dieser Ansatz eine Erklärung für die Entstehung der Creative Industries (s. Kap. 3). Zweitens beschreibt die Netzwerkforschung das Entstehen eng verwobener Industriegebiete, die aus kleinen, komplementären Unternehmungen (Firmen, Projekte, Aufträge) bestehen und gemeinsam Kreativität herstellen und verwalten. Und drittens besteht die Modekreation aus phasenspezifisch aktivierten Netzwerkteilen. Diese Aktivierung offenbart sich im Termingeschäft von Projekten, temporären Teams und befristeten Aufträgen. Aus repetierten, gepflegten Verknüpfungen entwickeln sich starke Beziehungen (wie beispielsweise zu Maschinen oder Produzenten), schwache Kontakte beziehen sich auf temporäre, entfernte Kooperationskontakte (beispielsweise Models, Fotografen, Grafiker).

Die Gesamtheit der an der Kreativitätsproduktion beteiligten Organisationen und Akteure besteht aus verschiedenen Hierarchien, Clusters und Netzwerken, die sich zu kleinen, temporären Projekteinheiten zusammenschließen. Becker nennt diese Produktionsnetzwerke »Art Worlds«, die temporäre Vereinbarungen der Kreativitätshervorbringung vor dem Hintergrund bestehender Strukturen koordinieren (Becker 1974: 767 f.). Ein Großteil der Arbeit in »Kunstwelten « beruht auf Projekten, weshalb diese Organisationsform auch für die Kreativitätsforschung bedeutend ist.

Kreativität muss in Verbindung mit kollektivem Organisieren studiert werden, denn »[...] art is social in the sense that it is created by networks of people acting together, and proposes a framework in which differing modes of collective action, mediated by accepted or newly developed conventions, can 
be studied« (Becker 1974: 775 Hervorh. der Verf.). In diesen Kooperationsnetzwerken finden Aktivitäten statt, die von der Erfindung der Instrumente bis hin zum Ticketverkauf für das Orchesterkonzert reichen (ebd.: 768). Die kollektive Zusammenarbeit ist die Konsequenz des hoch spezialisierten wie auch disparaten Wissens in der Kulturproduktion. So entsteht ein Netzwerk um die Produktion neuer Dinge, das nicht nur aus informalen, persönlichen Beziehungen besteht, sondern auch Verknüpfungen unter Vertrags- und Handelspartnern (Zulieferer, Dienstleister und Subunternehmer) erschließt. Das Produktionsnetzwerk konstituiert sich als Kommunikationsnetzwerk und ist für Kreativität bzw. die Fertigungswirtschaft von Neuerungen relevant, weil es die Vermittlung von Informationen begünstigt, als Rekrutierungsnetzwerk fungiert, die Suche nach neuen Ideen mobilisiert und die unternehmerische Offenheit für Neues verstärkt. Demzufolge wirken Produktionsnetzwerke als Mehrzweckressource (Powell/Smith-Doerr 1994 und 2003).

Der Fall Mode fristet ein Hybriddasein zwischen einer temporären Aktivitätsstruktur in Form von Projekten und einer steten Aktivitätsstruktur der Herstellung sowie der Formalstruktur der permanenten Organisation. Demzufolge bewegt sich die Produktion nicht durch eine Vielzahl formaler Organisationsdepartemente, sondern durch verschiedene Projekte, die nicht immer unter der Kontrolle der formalen Organisation ausgeführt werden. Je höher die Spezialisierung der einzelnen Unternehmen, desto größer deren Abhängigkeit untereinander; je diffundierter das Wissen, desto größer das Netzwerk.

Netzwerke - Powell (I990) spricht auch von »project markets « - entstehen zwischen Unternehmen und Projekten in Industriegebieten in Form von sozialen Produktionssystemen, die Eigeninteressen und soziale Verbindlichkeit bewältigen, langfristige Handelsbeziehungen auf Vertragsbasis errichten und das Vertrauen unter den im Netzwerk formal gleichgestellten, aber gegenseitig abhängigen Akteure errichten (Powell/Smith-Doerr 1994; Hollingsworth/ Boyer 1997). Uzzi bezeichnet diese Handelsverbindungen als »Produktionsketten« (Uzzi 1997). Weiter ist ein hoher Grad an Varianz, Unvorhersehbarkeit und Mobilität typisch für Produktionsnetzwerke:

These iproject markets are complex, dynamic, and uncertain. The participants in the film industry - producers, directors, cinematographers, actors, and musicians - appear at first glance to be highly mobile. They move from studio to studio, from one project to another, with few stable ties to any formal organization. [...] The organizations [...] are highly porous - with boundaries that are ill-defined, where work roles are vague and responsibility overlapping, and where work ties both across teams and to members of other organizations are strong. (Powell 1990: 308)

Solche kooperationsbasierten »Projektmärkte « bilden sich in Industriedistrikten ab, wie sie vor allem aus den Textil- und Modesektoren im Süden Deutsch- 
lands und im Norden Italiens bekannt sind. Das Beispiel der italienischen Bekleidungsindustrie steht für viele kleine, regionale Modeunternehmen, unter welchen sich nur wenige mit dem Endprodukt, viele aber mit Zwischenschritten und allgemeinen Dienstleistungen befassen. Diese Industriedistrikte werden in verschiedenen Aufsätzen als Beispiele für Koordinationsnetzwerke der Kreativität aufgeführt (Powell/Smith-Doerr 1994; Mora 2006; Aage/Belussi 2008). So auch bei Powell, der sich auf die Netzwerkformen der Textil- und Modedistrikte bezieht:

This production system depends on an extensive subcontracting system in which key technologies are developed in a collaborative manner. The subcontractors are also connected to overlapping inter-industry supplier networks. Theses linkages allow textile makers to benefit from the subcontractors experiences with customers in any one industry. All of these arrangements serve to strengthen the social structure in which textile firms are embedded and to encourage cooperative relations that attenuate the destructive aspects of competition. (Powell 1990: 309 f.)

Die kleinteilige Konfiguration dieser Industriedistrikte hat den Vorteil, dass aufgrund personeller, finanzieller und technologischer Flexibilität schneller auf die Wechselhaftigkeit und Dynamik kulturell-kreativer Märkte, wie beispielsweise die Mode, reagiert werden kann (ebd.: 3II). ${ }^{39}$ Auch Kadushin bespricht die kleinteilige, netzwerkartige Form der Kulturproduktion anhand der Bekleidungsindustrie:

A typical example given by economists is the women's high fashion garment industry. This is an industry characterized by a large number of fairly small producers huddled together in their mutual dependence upon designers, each other (for "stealing" ideas), specialty suppliers (of buttons, cloth, and so on), and a flexible labor supply of specialists who drift in and out of being workers or subcontractors. (Kadushin 1976: 772)

Kadushin spricht bei diesen Produktionsnetzwerken und Kooperationsteams von »Zirkeln«. Diese entstehen um geteilte Interessen und Bedürfnisse, sie aktivieren den Informationsaustausch untereinander, und Verbindungen wer-

39 | Powell bezieht sich hierbei auch auf die Verlagsindustrie, wobei er die Resonanz aktueller Paradigmen als erfolgsversprechende Besonderheit der wissenschaftlichen Verlagsarbeit hervorhebt: "The fortunes of a scholarly publishing house often depend more on the rise and fall of various academic paradigms than on the efficiency of a firm's internal operations" (Powell 1990: 307). Dieser Aspekt gleicht der Definition von Neuerungen in der Mode: Die Auffassung und lokale Interpretation internationaler Moden sind ein Indiz für soziale Einbettung und verweist auf die Rezeption aktueller Ereignisse und ihre Anpassungsfähigkeit. 
den durch Verträge »sichtbar« (I976: 77I f.). Zirkel kommen den Projektteams konzeptuell nahe, indem sie sowohl die Arbeitsteilung wie auch die gegenseitige Abhängigkeit ansprechen, als auch deren temporäre Aktivierung und die Herausbildung interner Kodifizierungen und Konventionen hervorheben.

\subsubsection{Soziale Netzwerke I: Orte des Vertrauens}

Für soziale Netzwerke spielen die Art und Struktur der Beziehung eine zentrale Rolle (Granovetter 1973). Empirische Studien haben gezeigt, dass sowohl die strukturelle Positionierung der Akteure (Zentrum versus Peripherie) wie auch die Qualität der Beziehung (starke versus schwache Beziehungen) einen Einfluss auf Kreativität haben.

Einige empirische Studien weisen auf die Qualität der Beziehungen, also deren Intensivierung und Stärke durch erhöhte Frequenz der Aktivierung, hin. Die Intensivierung bestimmter Kontakte hat aufgrund geteilter Interessen ein Clustering zur Folge, das Beziehungen stärkt und Vertrauen fördert. Eine Voraussetzung für das langfristige Bestehen der wechselseitigen Beziehungen der Kooperationsnetzwerke sind Vertrauensarrangements (Powell/Smith-Doerr 1994: 385). Dieses gilt als grundlegende Voraussetzung für die Ausarbeitung neuer Kollektionen in den Modefirmen, denn das ehrliche Feedback stärkt und verfestigt die neue Idee und unterstützt die Darstellung gegenüber Dritten (Uzzi I996; Gong u. a. 2013). Diese Intensivierung der Netzwerkkontakte kann aber auch Negativfolgen haben wie z. B., wenn sich Organisationen absondern und folglich weniger Kontakt mit strukturell interessanten (nicht redundanten) Netzwerkpartnern pflegen (Katz I982).

Schließlich zeichnet sich der Erfolg einer Modefirma durch den Mix der Beziehungsart, der Kohäsion und der Struktur aus, die allesamt zur Kreativitätshervorbringung beitragen (Uzzi I996; Perry-Smith 2006). Perry-Smith und Mannucci (2016) betonen die Netzwerkinhalte und Kognitionen, die bestimmte Kontakte aktivieren. Demnach sind je nach Phase mal starke, mal schwache Beziehungen wichtig. Dies führt zu einer bislang unerforschten Dynamik und hebt den relativen Einfluss aktivierter Netzwerkkontakte auf bestimmte Phasen des Kreativitäts- und Innovationsvorgangs hervor.

This malleability implies that individuals، ability to satisfy the needs of the different phases of the idea journey does not so much depend on the structure of their networks as it does on the subset of their networks that they cognitively activate. In addition, if network activation is dynamic and can change over time, then the network context that influences behavior also can change across phases of the idea journey. (Perry-Smith/ Mannucci 2016: 67, Hervorh. der Verf.) 
In Bezug auf Kreativität geht es weniger um die strukturelle Positionierung aktiver Kontakte, sondern vielmehr um die terminierte Aktivierung sogenannter subsets während bestimmter Phasen. Diese Subsets sind interdisziplinäre Arbeitsgruppen bzw. Projektteams, die nicht selten auch Organisationsgrenzen durchbrechen. Die Aktivierung und Terminierung der Netzwerkkontakte hängt von der Prozessphase und den darauf bezogenen Bedürfnissen ab. Das bedeutet, dass in den aktivierten Netzwerkteilen mal starke, mal schwache Beziehungen mobilisiert werden. Demzufolge setzen kreative Produkte kein spezifisches Netzwerk voraus, sondern die Projektteams schalten Netzwerkpartner ein und weisen ihnen Aufträge zu. Die wiederholte Aktivierung bestimmter Netzwerkkontakte reduziert Risiken und verstärkt das Vertrauen, wodurch Netzwerkteile - z. B. in Projektform - zu Orten des Vertrauens werden.

Über »strong ties« wird der Ressourcenzugang zu Neuem (Personal, Information, Infrastruktur) koordiniert, Probleme werden gelöst, Vertrauen hergestellt und detaillierte Informationen vermittelt. Nicht selten tragen eingebettete Netzwerkkontakte auch maßgebend zur gemeinsamen Problemlösung unvorhersehbarer Herausforderungen bei. In der Mode sind starke Beziehungen besonders typisch für die Vorstellung neuer Kreationen. Eine Partikularität dieser Beziehungstypen ist der »fine-grained information transfer« (Uzzi I997: 45 ff.), womit Uzzi die Vermittlung impliziter Informationen meint, die nicht über reguläre Wirtschaftsbeziehungen vermittelt respektive verstanden werden können. Neuentwickelte Modelle in einer Kollektion würden nicht von allen Kooperationspartnern gleichermaßen (>richtigく) verstanden, weshalb die Vorstellung der ersten Skizzen im engsten Kreis des Designteams stattfindet. Uzzi fasst die ambivalente Bedeutung eingebetteter und informativer Netzwerkkontakte zusammen:

On one hand, networks constituted of embedded ties [starke Beziehungen] benefit from trust, joint problem solving, and thick information exchange, which enhance coordination and resource sharing. On the other hand, networks composed of arm's-length ties [schwache Beziehungen] have wide access to information circulating in the market and an enlarged ability to test new trading partners. (Ebd. 1996: 684; Hervorh. der Verf.)

Während starke Beziehungen Vertrauen und Problemlösungsprozesse unterstützen, fördern schwache Beziehungen neue Kontakte. Diese Auffälligkeiten treffen auch auf die Modeindustrie zu: Vertrauen, gemeinsam gesetzte Ziele und enger Informationsaustausch sind die Folge starker Beziehungen; schwache Beziehungen haben demgegenüber den Vorteil, dass sie neue Informationen in die Organisationen bringen und Zugang zu neuen Ressourcen gewähren. 
In der Mode verschwimmen die Grenzen zwischen Zentrum und Peripherie, Konvention und Avantgarde. Die Kombination von Ideen aus verschiedenen Quellen ermöglicht den kreativen Umgang mit »neuem« Material (Uzzi/ Spiro 2005: 447). Netzwerke fördern den wirtschaftlichen Erfolg und machen die Neuerungen bekannt. Gleichzeitig sind sie Wege der Aufmerksamkeitssteigerung über die Organisationsgrenzen hinaus, über welche die Resonanz wie auch neue Stimuli in die Organisation hineintransportiert werden. Für die Umsetzung und anschließende Produktion sind strukturelle Merkmale wichtig, denn es geht um die Legitimität und Akzeptanz der neuen Kollektion und die Durchsetzung des Produkts.

\subsubsection{Soziale Netzwerke II: Innovationsorte}

Netzwerke sind Orte gespeicherter Ressourcen. Als solche werden sie für Kreativität und Innovation zum Spielplatz der Ideen und Einfälle, die dazu verleiten, soziale, institutionelle und symbolische Grenzen zu überschreiten und mit Konventionen zu brechen (Becker 2008 ; Burt 2004).

Andere empirische Studien verstehen Kreativität als Resultat strukturell verschiedener Positionen und der Kommunikation bzw. des Informationsaustauschs dazwischen. Entfernung zum Netzwerkzentrum kann Neuerungen und kreative Ideen fördern. Gatekeeper besetzen strukturell interessante Positionen im Netzwerk, denn sie überbrücken kaum vernetzte Orte und führen so Ressourcen zusammen, die sonst nicht in Kontakt kämen (Burt 2004; Uzzi/ Spiro 2005; Perry-Smith 2006). Gatekeeper fungieren als Intermediäre (von Burt auch Broker genannt [2004]), die zwei organisationale Bereiche (Projekte, Unternehmen, Felder, Welten) auseinanderhalten wollen, zwischen ihnen aber temporäre Brücken schlagen, um so Talent, Können, Kapitalgeber und Vertreiber miteinander zu verknüpfen (Caves 2002; Tschang 2007; Perry-Smith/ Mannucci 20I6). Gatekeeper besetzen deshalb auch die Rolle, über originelle Ideen und deren Verbreitung zu entscheiden (Elsbach/Kramer 2003). Aufgrund der strukturellen Positionierung im Netzwerk wissen Broker auch über Aktualitäten und Moden Bescheid (Foster/Ocejo 20I5: 4I6). Demzufolge hat das Beobachten von Gatekeepern und der durch sie verbreiteten Informationen eine legitimierende Wirkung auf organisationales Handeln (Perry-Smith/ Mannucci 20I6).

Über die temporäre Brücke wird Zugang zu neuwertigen und unbekannten Informationen sowie Ressourcen erschlossen. Zusätzlich rückt eine andere Form des Brokers, die »Amphibien« (Eigenbrötler und Avantgardisten), zunehmend ins Scheinwerferlicht der Kreativitätsforschung. Gegenüber Gatekeepern schaffen sie es, in zwei unterschiedlichen Welten Fuß zu fassen und diese Überlappung künstlerisch zu nutzen, denn in der Peripherie des Netzwerkes (und nicht in dessen Zentrum) werden Normbrüche und unkonventio- 
nelles Verhalten eher toleriert (Cattani/Ferriani 2008: 827 f.). In entfernten Netzwerkpositionen führen amphibische Künstler die Avant-Garde an und können sich trotzdem den Zufluss neuer Informationen für innovative Ideen wie auch den Zugang unbekannter Handelskontakte zu Nutzen machen (Jones u. a. 20I6; Patriotta/Hirsch 20I6). Kreative Ideen sind gemäß Burt die Folge der Kapazitäten zwischenorganisationaler Räume:

Across the clusters in an organization or market, creativity is a diffusion process of repeated discovery in which a good idea is carried across structural holes to be discovered in one cluster of people, rediscovered in another, then rediscovered in still others - and each discovery is no less an experience of creativity for people encountering the good idea. (2004: 389)

Burt beschreibt Kreativität hier als repetitives, kontextuelles Erlebnis des Entdeckens guter, neuer Ideen. Sie ist jedoch auf Gatekeeper, deren Bewertungen und Diffusionsmöglichkeiten angewiesen. Die Entdeckung und Gutheißung einer kreativen Idee ist jedoch von unterschiedlichen Brokern abhängig, denn die einen können sie strukturell herstellen, jedoch nicht vertreiben (Amphibien), während die anderen sie aufgrund der zentralen Position im Netzwerk nicht erkennen, aber anpreisen können (Gatekeeper):

The fringe is where divergent ideas thrive, yet it also is the place where they risk remaining invisible, and their exploration unexploited, because, no matter how original the insight, the label of ,creativer depends on gatekeepers who can support and legitimate it. (Cattani/Ferriani/Colucci 2015: 88)

Grenzräume zwischen Netzwerken, in denen Kreativität floriert und woraus gute Ideen entstehen, bergen den Nachteil der Unsichtbarkeit der Idee. Diese Aufmerksamkeit führt zu der überlebenswichtigen Sichtbarkeit. Bei neuen Ideen wie beispielsweise avantgardistischen Moden oder textiltechnischen Neuerungen können Modenschauen oder Magazinbeiträge zu dieser Sichtbarmachung beitragen.

Organisationen können sich aufgrund der Ressourcen hin zur Peripherie öffnen. Die Grenzen werden durchlässig und die Umwelt wird von einem Ort der Ressourcenakquise zum Lern- und Innovationsort (Cattani/Ferriani 2008; Dokko/Rosenkopf 2010)..$^{40}$ Soziale Netzwerke spielen auch in der Hervorbringung von Kreativität eine zentrale Rolle, denn da fungieren sie als Rekrutierungspool für Personal (Godart/Shipilov/Claes 20I4 und Godart u. a. 20I5),

40 | Grabher (2001) spricht hierbei von "trading zones", die sich bei wiederholter Aktivierung bestimmter Netzwerkkontakte ergeben und zum Schauplatz verschiedener organisationaler, wirtschaftlicher und künstlerischer Interaktionen werden. 
als Plattform für Informationen (Aage/Belussi 2008) und Schlüsselstelle neuer Ideen (Burt 2004). Das Netzwerk wird zum Innovationsort (Powell/Koput/ Smith-Doerr I996; Hasse/Passarge 20I5). Kreativität ist dann also das Resultat erweiterter organisationaler Weltsichten und Perspektiven an der Schnittstelle von Organisation und Umwelt.

Über »den Fall« der Pariser Modeindustrie ist bekannt, dass sich die Modedesigner französischer Couture-Häuser vornehmlich als Teile eines Netzwerks und weniger als (ehemalige respektive gegenwärtige) Angestellte eines Unternehmens verstehen und deshalb Informationsflüsse nach außen aufrechterhalten und gegenseitig bedienen. Diese Mobilität der Angestellten wird als gewinnbringende Verbindung zur Konkurrenz dargestellt: »Thus organizations that access heterogeneous information from their environment, and those that can most favourably influence their audience's perceptions, « so das Fazit der Autoren, »are the ones that are more likely to succeed in producing creative output« (Godart/Shipilov/Claes 20I4: 378). Kreativität ist folglich auch Produkt der Instrumentalisierung und Kombination verschiedener Informationsquellen. Bleiben Verbindungen zur Konkurrenz aus, erschöpfen die Ressourcenkontakte und die Informationsflüsse versiegen. Dann besetzt der Modedesigner nicht länger eine Gatekeeper-Position bzw. verbindet keine getrennten »Welten« miteinander.

\subsection{Kreativität innerhalb der Organisation}

Als Gegenstand der Sozial- und Verhaltenspsychologie wird Kreativität in Ansätzen zu Generierungs- und Förderungstechniken weiterentwickelt. Diese konzeptuellen und analytischen Richtungen der Kreativitätsforschung haben vor allem im Bereich des Managements mächtig Anschluss gefunden (Bilton 2007). In diesem Kapitel wird auf die Literatur der Kreativitätstechniken eingegangen, die später (in Kap. 6) teilweise wieder aufgegriffen und mit empirischen Beispielen illustriert werden.

Innerhalb der Betriebe geht Kreativität aus der organisatorischen Synthese zwischen verschiedenen, situativen Variablen im Kontext der Entwicklung und Produktion von neuen Kreationen hervor (Harvey/Kou 20I3; Madjar u. a. 2002; Carlsen u. a. 20I3; Oldham/Cummings I996). Resultierend aus der Divergenz zwischen Bestehendem und Neuem, entsteht eine Form der »kombinatorischen Kreativität« (Tschang 2007) oder »architektonischer Innovation« (Henderson/Clark I990). Die Managementforschung hat ein besonderes Interesse an der Kontrolle von Leistungsanstrengungen, weshalb Techniken und Praktiken als Methode zur Kreativitätsgenerierung in den Fokus rücken, die sich auf das intraorganisationale Koordinieren kreativer Prozesse spezialisieren. Dabei kann entweder das Problem oder die Lösung vorgegeben sein 
(Gilson/Madjar 20II), und der Stimulus der Kreativität interner oder externer Natur sein (Unsworth 200I). Slavich und Svejenova (20I6) analysieren unter dem Begriff »Managing Creativity« sechs verschiedene Schwerpunkte, darunter Kreativität als Ergebnis (von Neuheit, Originalität oder Nutzen) oder Kreativität als organisationalen Prozess (aus Kooperationen, Zusammenarbeit und Interaktionen, Abb. I). Kreativität zeigt sich im Effekt von Modifikationen, Transformationen, Erneuerung und der Aufarbeitung zwischen oder innerhalb von Organisationen:

In this view, creativity is about change through constant and dynamic processes of modification, transformation, renewal, and regeneration, which provide an outcome with an impact [...]. The focus in these definitions is not on a process of generation of something different, but on the substantial impact that an outcome produces in an organization or a field. (Slavich/Svejenova 2016: 244)

Am häufigsten sind Kreativitätsdefinitionen, die sich auf beide Kategorien beziehen..$^{\mathrm{I}}$ Da Konformität keinen Effekt auf das Feld hat - sie ist kaum wahrnehmbar (Goncalo/Duguid 20I2) -, stellt diese das Gegenteil von Kreativität dar.

Katherine Chen (2009 und 2012) kritisiert die psychologisierende Dominanz in kreativen Prozessen und argumentiert, dass sowohl totale Formalisierung wie auch absolute Freiheit kreative Leistungen einschränken würden. Ihr Fokus bearbeitet zwei Extreme: das Über- und Unterorganisieren. $\mathrm{Zu}$ viel Formalstruktur, Rationalisierung und Gewaltkontrolle reduzieren Experimentierfreude, die ein besonders großes Potenzial für kreative Lösungen fördert: »In short, excessive rationalization and coercive control can generate over-organizing that discourages experimentation and encourages conformity, thereby constraining creativity« (Chen 20I2: 629). Hingegen führen wenig Formalstruktur, Kontrolle oder Leitlinien zu »unterorganisierten « Teams, die wiederum in Hierarchien und richtungsweisenden Regelwerken nach Halt suchen oder die Organisation verlassen. Weder übernoch unterorganisierte Sozialräume begünstigen das kreative Zusammen-

41 | Slavich und Svejenova analysieren vier Prozesse, in die sich die aktuellen Kreativitätsdefinitionen kategorisieren Iassen (2016: 242 ff.): darunter die Synthese als kognitiver Prozess aus den Erfindungen, Problemlösungen und Verbesserungen entstehen, der sich aber weiterhin Routinen bedient und innerhalb bekannter Verfahrensweisen weiterentwickelt; das Engagement als Verhaltensprozess, der die motivationale Einwilligung zu vertiefter Auseinandersetzung des Individuums meint; die Interaktion als relationaler Prozess, der auf die Verbindungen und Kollaborationen verweist und auf die Kollektivität konzentriert; oder die Kreation als organisationaler Prozess, dem die Autorinnen sich umfassend zuwenden. 
spiel in Organisationen. Kreativität entsteht folglich im Zustand relativer Ausgewogenheit verschiedener, scheinbar kontradiktorischer Erwartungen. Dieses Balancieren stellt nicht nur die Organisation, sondern auch die Individuen vor eine Herausforderung. Dennoch sind es zumeist bürokratische, »überorganisierte « Organisationen, welche größeren Schwierigkeiten begegnen, Kreativität zu ermöglichen, da Rationalisierungen und genaue Zielvorschriften die Stabilität, Uniformität und die Berechenbarkeit des Produktionsprozesses erhöhen, Nonkonformität unterbrechen und die Vielfalt der Aktivitäten einschränken (Chen 20I2: 629). Die Dezentralisierung von Wissen durch eine erhöhte Personalfluktuation hat einen Einfluss auf neue Ideen und deren Verbreitung, was sich wiederum positiv auf »überorganisierte« Strukturen auswirkt (20I2: 63I). Während die eisernen Strukturen der Bürokratie kreative Prozesse stark einschränken und in ihrer Kapazität beschneiden, rückt Chen das Potenzial von Routinen in den Vordergrund der organisationalen Kreativitätsforschung: »routines can correct for under-organizing by orienting activities. Routines provide a basis for improvisation, leading to variation [...]. In carrying out routines, members may creatively recombine parts of these routines to achieve desired ends « (Chen 20I2: 632). Routinen werden zunehmend zur konzeptuellen Basis für kreative Handlungen, d. h. Improvisationen und Rekombinationen, und somit für Variation und Kreativität (s. Kap. 6.I). 
Abb. 1: Zentrale Kategorien der Kreativität: Beschreibungen, Definitionen, repräsentative Studien

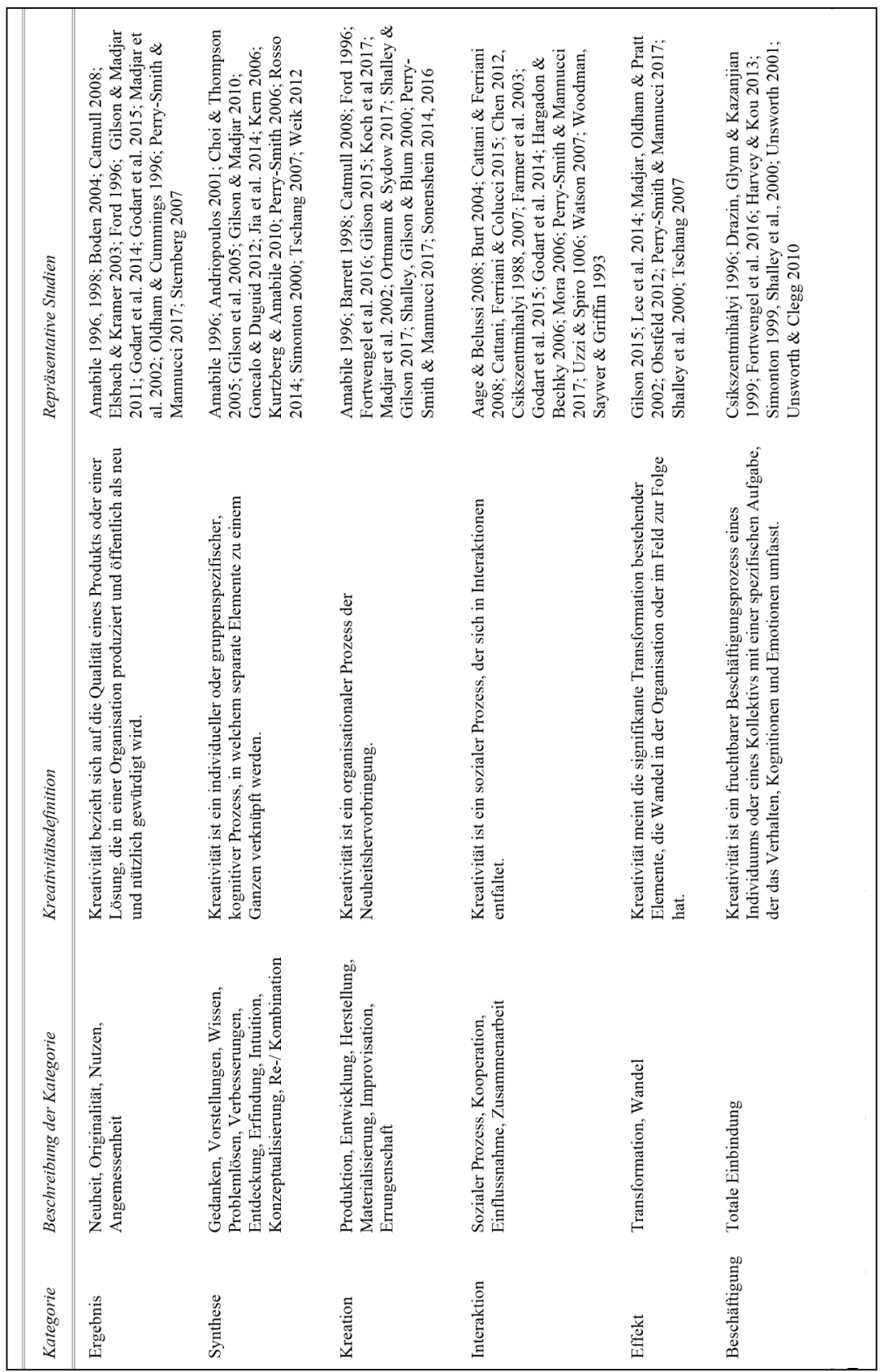

Quelle: Eigene Darstellung nach Slavich/Svejenova 2016: 241 
Eine Organisationskultur, die den spielerischen Umgang mit neuen und bestehenden Arbeitspraktiken fördert, erhöht die Kombinationsmöglichkeiten und stimuliert so die Entstehung kreativer und innovativer Ideen (Carlsen u. a. 2013). Rekombinationen sind daher eine gängige Generierungstechnik für Kreativität: »the manipulation and recombination of objects in novel and useful ways to solve problems « (Sonenshein 20I4: 8I4), »the recombination of existing ideas from different sources into new products « (Tschang 2007: 989), »new connections between relatively unchanged components « (Obstfeld 2012: I589) und schließlich: »This novelty represents a recombination of existing elements and styles that differentiates, but does not break existing artistic and aesthetic conventions « (Lampel/Lant/Shamsie 2000: 266). Die Rekombinationstechnik verspricht, bestehende Elemente neu zusammenzustellen, um dadurch $\mathrm{zu}$ alternativen Ideen zu gelangen oder diese andersartig zu inszenieren, um Verständlichkeit zu generieren und den Markterfolg zu erhöhen (ebd. 2000). Bartel und Garud (2009) erachten die Praxis der Rekombination als zentrale Übersetzungstechniken gängiger Elemente in lokale Kontexte, die als typischer Arbeitsverlauf in Organisationseinheiten kultiviert werden sollte. Die für kreative und kulturelle Produktion typische Unsicherheit oder Ambivalenz bezüglich Markt und Nachfrage wird somit nicht als Problem, sondern als vieldeutige und fruchtbare Ressource mit in den Prozess eingebaut (Vangkilde 20I3: 90). Sonenshein (20I4) wendet den Terminus »creative resourcing« an, um zu erklären, wie bestehende Materialien und eingeschränkte Ressourcen zu kreativen Lösungen kombiniert werden können, die im Publikum Anklang finden. Versteht man Kulturproduktion als Anschluss an Vorgängerbeispiele, dann wird kreativer Ausdruck zum Produkt vieldeutiger und komplexer Erneuerungsprozesse (Bartel/Garud 2009: I09; Moeran/Christensen 20I3: 19).

Jazzimprovisationen sind ein Beispiel solcher Imitations- und Kombinationssysteme: "Jazz is the only music in which the same note can be played night after night but different each time« (Ornette Colman zit. nach in Barrett I998: 620). In der Organisationsforschung dient der Jazz als Beispiel für die Wissensvermittlung und die Improvisationsgabe, wodurch ein flexibler Routinebegriff plausibilisiert wird. Jazzmusiker produzieren etwas Neues und Andersartiges, indem sie das Gelernte und Einstudierte mobilisieren (Barrett I998: 608 ff.; Hargadon/Bechky 2006: 485). Die Improvisation ist ein Moment musikalischer Synchronisation, indem die Jazzbands mithilfe geteilten Wissens, geteilter Beobachtung (während der Performance) und aufgrund unterschiedlicher Interpretationsweisen die Situation kontinuierlich neu verhandeln. Weiter sind die Musiker der Jazzbands darauf spezialisiert, die Kernkompetenz trotz situativer Variationen zu wahren:

Good things can happen when people jump in and act even when all plans are not complete and elegant. Rather than over-rely on pre-planned strategies and canonical 
job descriptions, acknowledge members capacity for bricologe [sic!] and pragmatic reasoning, their ability to juxtapose, recombine, and reinterpret past materials to fashion novel responses. (Barrett 1998: 619)

Praxis- und Technikwissen untermauern die Rekombination bestehender Praktiken. Werden flexible Routinen und freies Kombinieren von stabilen Strukturen der Organisation begleitet, können sie zu organisationaler Kreativität beitragen. Folglich beruht Organisationsform auf wenig Planung und Hierarchie, wird aber durch redundante Information begünstigt. Das Vorteilhafte regelmäßiger Interaktionen ist das Kondensieren kollektiver Augenblicke, die zur Überwältigung von konventionellen Grenzen und zum Ausschöpfen kreativer Potenziale führen.

\subsection{Fazit II: Kreativität als Resultat MEHRLAGIGER SPANNUNGSVERHÄLTNISSE}

In der Organisationsforschung bedeutet »kreativ« eine mehrlagige Qualität, eine situativ-kontextuelle »sliding category« (Moeran 2009: 98I). Unterschiedliche duale Organisationsbedingungen haben einen Effekt auf die Kreativitätshervorbringung bzw. die Entwicklung und Produktion von Neuerungen: Repetition und Entdeckung (Obstfeld 20I2: I572), Ästhetik und Funktion (Moeran/Christensen 2013), Prozess und Resultat, Individuum und Kollektiv, Freiraum und Einschränkung (Ortmann/Sydow 20I8) oder Permanenz und Temporalität (Slavich/Svejenova 20I6: 245 f.). Folglich ist das zentrale Problem des Managements von Kreativität das Spannungsverhältnis, Variation zu erhöhen und operative Abweichung zu minimieren:

Thus, the core of the creativity paradigm is enhancing variation to optimize the fit between team efforts and outcomes, whereas the core of standardization is minimizing variance to ensure consistent operations. This antithesis presents teams and ultimately, organizations, with an interesting dilemma. On the one hand, it is desirable for teams to be creative in performing their work; on the other hand, it is desirable for them to adhere to standardized work practices. (Gilson u. a. 2005: 523)

Die Herausforderung für Organisationen der Creative Industries ist das Dilemma und die Paradoxie des Organisierens zwischen der effizienten Ausrichtung an Vergangenem und der kreativen Gestaltung der Neuheit. Um dem entgegenzuwirken, werden nicht Techniken und Praktiken, sondern auch Ressourcen wie Personal oder Informationen unterschiedlich kombiniert. Die Kombination der Ressourcen hat einen großen Einfluss auf neuartiges Den- 
ken und die Generierung kreativer Ideen. Neuerungen entstehen durch den Zugang zu abweichendem Wissen, das durch die Übersetzung in situative Eigenheiten und Ziele lokal zugänglich gemacht und im organisationalen oder individuellen Repertoire gespeichert wird (Czarniawska/Joerges I996; Oldham/Cummings I996; Hargadon/Sutton I997; Madjar u. a. 2002; Cattani/ Ferriani 2008). $4^{2}$

Wie aus dieser Literaturübersicht hervorgeht, ist das Feld der Kreativitätsforschung von einer Vielzahl von theoretischen, methodologischen und empirischen Erklärungsansätzen durchsetzt. Ein organisationstheoretischer Blick schärft das konzeptuelle Instrumentarium zusätzlich. Versteht man Kreativität als organisiertes Problem, rücken interorganisationale Netzwerke im Sinne der »loci of innovation « und intraorganisationale Routinen, Technologien und Teams als relevante Konzepte der Kreativitätsforschung in den Vordergrund (Powell/Koput/Smith-Doerr I996).

Das Organisieren von Kreativität basiert auf Kooperationen, die durch Routinen strukturiert werden. Routinen werden hierzu nicht als rigide, statische Verfahrensregeln begriffen, sondern als flexible Musterhandlungen, die in situ Deutungen zulassen und dadurch Veränderung (z. B. Rekombinationen und abweichende Interpretationen) ermöglichen. Routinen sind die mikrosoziologische Einheit, die sich zu einer größeren (Projekt-)Aufgabe zusammenfügen. Diese Aufgaben liegen im Zentrum der (heterogenen) Teams, die mit der Aufgabe betraut werden. An dieser Stelle muss betont werden, dass Talent und besondere Fähigkeiten betreffend Ideenfindungsvermögen unbestreitbar sind. ${ }^{43}$ Da dieser >kreative Geist< jedoch nur einen Teil der Voraussetzung für Kreativität in Organisationen darstellt, muss diese Konzeptualisierung mit sozialen und organisationalen Aspekten ergänzt werden. Die vorliegende Arbeit konkretisiert Kreativität als kollektiven Prozess. So schreibt Burow (I999): »Kreativität gibt es nur im Plural!« und verleiht der kollektiven, kooperativen Kreativitätsdefinition Nachdruck (Bourdieu I993; Moeran 2009; Obstfeld 20I2).

42 | Für die Modeindustrie sind Archive besonders gewinnbringend, denn als intersubjektive, soziale Gedächtnisse speichern sie Aktuelles und machen es unabhängig von ihrer Aktualität zugänglich. Mittels archivierter Daten kann in Langzeitstudien die Diffusion und Adaption von Trends über Zeit modelliert werden (Godart forthcoming).

43 | Amabile spricht von der Bedingung des " good fit" und der "innate abilities" und meint damit, dass gewisse Personen besonders gut zu bestimmten Situationen, z. B. Schach spielen oder Musik komponieren, passen, jedoch diese angeborenen Fähigkeiten nicht immer ausreichend sind, um eine bedeutende, kreative Leistung zu erbringen (Amabile 1983: 361). 


\section{Die Organisation der Modekreation}

In diesem Kapitel werden die organisatorischen Voraussetzungen der Kreativität in der Modebranche ausgeführt. Wie die Feldforschung in den Modeunternehmen gezeigt hat, ist Kreativität kein Wort, dessen sich die Mitglieder im Unternehmen täglich bedienen. Deshalb stellt sich die Frage, was in Modefirmen denn eine Rolle spielt (und welche?), wenn nicht Kreativität? In diesem Kapitel werden organisatorische Theoriekonzepte aus der Routineforschung, dem Kontingenzansatz und der Projektforschung vorgestellt und eine Verknüpfung mit empirischen Themen des Feldmaterials hergestellt. Die Konzepte und organisatorischen Prozesse fasse ich als technologische Aspekte der Kreativität zusammen.

Der Prozess der Kollektionsentwicklung und des Designs neuer Kleiderkollektionen ist ein wiederholter und deshalb erwarteter Vorgang: Meetings folgen Meetings, Deadlines jagen Deadlines und Routinen und Wiederholungen ergänzen sich gegenseitig zu eingespielten, trainierbaren und schließlich generationenübergreifenden Handlungsmustern, anhand derer ein neues Kleidungsstück designt und produziert wird (Bouty/Gomez 20I6). In diesem Hervorbringungsprozess ist Kreativität zwar beabsichtigt und erwartet (Unsworth 200I: 29I), dennoch spielt dabei manchmal auch der glückliche Zufall - »serendipity « genannt - eine Rolle (Simonton I999). Damit ist gemeint, dass sich Kreativität zwischen der sicheren Gewissheit der Überraschung und der ungeplanten Störung der Zuverlässigkeit bewegt. Kreativität wird dadurch zwar voraussagbar, nicht aber berechenbar. Es komplettieren und kompromittieren sich Strategie genauso wie Spürsinn; Design genauso wie glückliche Zufälle; und Planung genauso wie Emergenz zur Hervorbringung von Kreativität in der Mode. Auffällig ist, dass die Mode es schafft, trotz nicht linearer Vorgänge und vielzähliger Kompromisse Kreativität dennoch ziemlich zuverlässig hervorzubringen (Catmull/Wallace 20I4; Pratt u. a. 20I5). Diese Art von Kreativität wird selten als kommunikativ ausgewiesen und bleibt in Form von Tüfteln projektintern relevant bzw. für das Publikum unsichtbar.

In den Modefirmen haben sich diese Voraussetzungen auf dreierlei Weise gezeigt: Erstens stützt sich Modedesign auf Routinen, die nicht alle gleichzeitig und vor allem geografisch disparat aktiviert werden, aber schließlich alle 
zur Entwicklung und Produktion der Kreativität in der Mode beitragen. Zweitens wird der Betrieb vor der Umwelt geschützt, um durch den operativen Kern konstante Wertschöpfung zu gewähren, während Überraschungen, Emergenz und glückliche Zufälle ebenso als Bestandteil der Kreationen erwartet werden. Durch die partielle Öffnung der Organisation wird Veränderung ermöglicht. Dadurch treten nicht nur Ideen und Inspirationen aus der Umwelt in die Organisation, sondern auch Personal, Ressourcen und Informationen. Deshalb ergeben sich drittens aus den koordinierten Interaktionen der Projektmitglieder unvorhersehbare Probleme, da weder die Sprache noch die erklärten Ziele oder die persönlichen Motive geteilt werden. Diese Voraussetzungen verlangen sowohl Koordination als auch Organisation und verbinden Neuerungen und Routine auf selbstverständliche Weise.

\subsection{Verlässlichkeit und Variation: Routineforschung}

Routinen sind mehr oder weniger stabile und iterative, mikrosoziologische Einheiten. Für die Analyse der Kreativitätshervorbringung in der Modebranche stellen Routinen die mikrosoziologische Grundlage für die serielle Ausarbeitung und Produktion neuer Kleidermodelle dar. Rekursivitäten und Wiederholungen entstehen aus Handlungsmustern, die sich von Mal zu Mal, von Wiederholung zu Wiederholung verändern. Child (I997) spricht deshalb von Routinen als Sedimentation der Erfahrung von Organisationen. Auf der Mikroebene rücken individuelle und organisationale Tätigkeiten in den Fokus soziologischer Analysen. Die Routineforschung studiert, »what individuals or organizations do separately from what they [or what we, the audience] mean by doing it« (Powell/Colyvas 2013: 294). Deshalb bringt die Routinetheorie die Kreativitätsforschung voran, weil sie den Fokus auf die Wiederholungen im organisationalen Alltag richtet und die fundamentalen Komponenten des Organisierens beleuchtet (Berger/Luckmann I99I; Hasse/Schmidt 2008). Erstens werden durch die Rekombination von Routinen die organisationalen Fähigkeiten reproduziert und verstetigt; zweitens adressieren sie die Mehrdeutigkeit und Unsicherheit aus der Umwelt, indem sie Bekanntes reproduzieren und mit Neuem kombinieren; und drittens lassen sie aufgrund ihrer flexiblen Deutungsweisen genügend Handlungsfreiraum. Basierend darauf machen Routinen Neuerungen möglich.

Das Feld der Mode lässt sich als Sektor beschreiben, der maßgebend darauf basiert, dass ähnliche Produkte und Vorhaben schablonenhaft (re-)produziert werden und somit deren Effizienz und Erfolg gesteigert wird (Davies/Brady 2000; Brady/Davies 2004). Demnach perpetuieren Modefirmen die kontinuierliche und wiederkehrende Erneuerung der Mode. Infolgedessen werden Kollektionen zu »going concerns« (Ventresca/Kaghan 2008), wobei das »going« 
auf die permanenten Operationen verweist und die »concerns « ihre temporäre Form meint. Dafür ist das in Routinen gespeicherte Wissen relevant, welches verwahrt und rezykliert wird. Aus diesem Grund sind Routinen zentrale Aspekte der Kreativitätsforschung.

Konzeptuell beziehen sich Routinen auf Institutionen, Normen und Konventionen oder individuelle Gewohnheiten, Programme, Skripte oder werden etwa als organisationale Gene bezeichnet (Becker 2008; Hodgons 2008). Brian T. Pentland und Henry H. Rueter skizzieren (I994) ein grammatikalisches Routinekonzept, in dem die Syntax die Kombination einzelner Komponenten strukturiert. Martha S. Feldman und Brian T. Pentland erweitern Routinen zur Quelle von Flexibilität und Wandel (2003). Dieses Routinekonzept bietet mächtig Anschluss für die Auseinandersetzung in der Kreativitätsforschung, so z. B. zu Themen wie organisationalen Praktiken in den Kulturindustrien (Lampel/Lant/Shamsie 2000), Kreativität und Standardisierung (Gilson u. a. 2005), Kreativität und Routinen als Dualstruktur von Neuheit und Bekanntheit (Sonenshein 20I6) und schließlich kreative Praxis durch Einschränkung und beabsichtigte Passivität (Ortmann/Sydow 20I8). Dieses Dualitätsargument zwischen Kreativität und Routine wird dadurch bestärkt, dass »regularly acting creatively produces structures that inevitably shape subsequent creative acts « (Sonenshein 20I6: 740). Kreationsprozesse sind rekursive Tätigkeiten, die ineinandergreifen und sich formen. Diese Auseinandersetzung bietet einen neuen Erklärungsansatz für Kreativität durch Standardisierung und Routine und präsentiert anstelle eines mystifizierenden Volksmärchens (kreatives Genie, Eureka-Momente) ein effektives Konzeptpaar. Letztendlich haben die Routine- und Kreativitätsforschung ein wechselseitiges Interesse an Theoriekonzepten und entwickeln gemeinsame Erklärungsmöglichkeiten.

Inzwischen ist die standardisierte Herstellung von Kultur- und Kreativgütern auch in der Forschung der Creative Industries Realität, denn auch besondere, einzigartige Aufgaben entstehen durch routineartige Elemente (March/ Olsen I989; Bakker 20I0: 478). Für Becker ist die Professionalisierung der Ausbildung, genauer »the supply of interchangeable human parts«, ein Hinweis auf Aufgaben, die sich mittels Routinen ausführen lassen: »When you can count on replacing people with others just as good, you can carry on artistic work in a routine way« (Becker 2008: 78). Folglich wird auch in der Kreativitätsforschung das Argument laut, dass standardisierte Verfahrensmuster und Einschränkungen eine bedeutende Rolle spielen, die in Form einer »well-defined creative challenge« den Produktionsprozess gestalten (Rosso 20I4: 570). Die Ressourcenverwaltung, die Koordination von Routine und Neuheit in Form von kreativen Projekten, Konformität mit bestehenden Regeln oder Standardisierung der Arbeitsprozesse wirken sich nicht nur negativ, d. h. einschränkend, sondern auch positiv, d. h. aktivierend auf die organisationale 
Kreativitätshervorbringung aus (Gilson u. a. 2005; Kern 2006; Obstfeld 20I2; Sonenshein 20I6).

\subsubsection{Routinen als verlässliche Leistungsvermögen}

Routinen sind Leistungsvermögen, die situativ abgerufen werden, zu akuten Problemlösungen beitragen, periodisch wiederkehrend sind und sich im Laufe der Zeit entwickeln. Obwohl Routinen an situative und lokale Gegebenheiten angepasst werden, bestehen sie aus mehreren Subroutinen, die ausschließlich durch das kollektive Zusammenspiel in Kraft gesetzt werden.

Ältere Konzepte beschreiben Routinen als rigide Einheiten, die das Handeln regulieren und Intuition unterbinden, andersartige Alternativlösungen wie auch den Wandel in Organisationen einschränken oder sogar verunmöglichen. Cohen $u$. a. einigen sich auf folgende Routinedefinition (I996: 683): $» \mathrm{~A}$ routine is an executable capability for repeated performance in some context that [has] been learned by an organization in response to selective pressures $[\mathrm{A} / \mathrm{N}]$.« Die Autoren präzisieren Routinen als regelmäßige, repetitive und kontextuell bedingte Handlungsweisen, die durch einen situativen Stimulus eine schematische Reaktion auslösen. Dieses rigide Stimulus-Reaktionsmodell versteht sich auf eine Konzeption, die das Gegenteil von Wandel und Dynamik meint.

Auch evolutionsökonomisch werden Routinen als die Fähigkeit verstanden, komplexes und effektives Verhalten auf gleiche, standardisierte Weise zu handhaben und dadurch Überlebensvorteile zu generieren (Nelson/Winter I982). Infolgedessen dienen Routinen der Verhaltensorientierung, denn das Verhalten der Vergangenheit wird in Routinen gespeichert und führt zu Annahmen über die Verhaltensmöglichkeiten der Zukunft. Das bedeutet, dass Routinen Auskunft über Verhalten und Handlungen geben, indem sie auf kollektiv geteilte Tätigkeiten und Programme verweisen (Pentland/Rueter I994: 487).

March (I99I) ergänzt dieses Model um den Aspekt der »exploration«, der Suche nach Variation über Risikofreude, Experimentieren, Spiel, Flexibilität, Entdeckergeist und Innovation, die kurzfristig nutzbringend sein kann. Die Exploration ist damit das Gegenstück der rigideren »exploitation«, die eher der Entscheidungsfindung und effizienteren Produktion dient. Gemäß March benötigen Organisationen sowohl Anpassungsfähigkeit als auch Ausnutzung, sie variieren jedoch in der Form der Ressourcenhandhabung, Zielsetzung, Regeln und Praktiken. Der Ausschluss der Exploration führt, so March, zum suboptimalen Zustand des stabilen Gleichgewichts. Ein solches Gleichgewicht ist aufgrund möglicher Lock-in-Effekte schlecht. Routinen begünstigen Lock-in-Effekte (March/Simon I958). Um organisationales Überleben und Prosperität beizubehalten, bedarf es einer Balance zwischen »exploration« und 
»exploitation«, also zwischen rigidem-routiniertem und flexiblem-routineähnlichem Handeln (Obstfeld 20I2).

Das rigide und statische Routineverständnis wurde mit den Jahren um dynamische Elemente ergänzt. Jüngere Studien verstehen Routinen als weit mehr als abstrakte und mechanistische Regelwerke der Mikrosoziologie. Tatsächlich sind Routinen kollektive Aktivitäten und kognitives Wissen (Hodgons 2008: 19), die Funktionen und Fähigkeiten umfassen, die zwar gespeichert und abgerufen werden können, sich aber sowohl in ihrer Ausführung als auch im zugrundeliegenden Stimulus unterscheiden (Feldman/Pentland 2003). Die Routinenforschung weist auf zwei praktische Vorteile hin: Erstens speichern sie Zugang zu Wissen und bieten zweitens auch günstige Lösungsansätze bei Konflikten an (Nelson/Winter 20I4: 30). Um sich von der Trägheit institutionalisierter und der Reproduktion bekannter Organisationsformen loszulösen, werden Routinen mit Neuheit ergänzt und verlieren dadurch ihre Rigidität (Nelson/Winter I977 20I4).

Diese Perspektive auf habitualisierte, alltägliche Arbeitsabfolgen lädt ein, die lokale Übersetzung sozialer Erwartungen zu beobachten respektive die individuellen Interpretationen sozialer Praktiken in spezifischen Situationen zu untersuchen (Powell/Colyvas 20I3). Die Wahrnehmung und Interpretation des Stimulus bestimmen dann, ob Routinen überhaupt und wenn, ob kollektiv oder individuell ausgeführt werden. Die kollektive Ausführung ist die Summe aktivierter Akteure und Routinen, ähnlich neuronaler Gehirnaktivitäten, in denen »people as neurons and organizations as a brain « wirken (Fiol 2005: Kap. »connectionist architecture«). Routinen werden in Organisationen wechselseitig aus individuellen Handlungen und kollektiven Programmen konstituiert. Diese holistische, kollektive Qualität wird zum Gegenstand jüngerer Konzeptualisierungen (Pentland/Rueter 1994: 484).

So wird beispielsweise von jüngeren Beiträgen der organisationstheoretischen Kreativitätsforschung konstatiert, dass routineähnliche und musterhafte Tätigkeiten die Basis für Wandel oder Innovationen sind (Feldman 2000; Ventresca/Kaghan 2008). Routinen sind weit beugungsfähiger, als bis anhin vermutet, und oft verändern sie sich durch den Wandel, den sie nicht selten selber initiieren (Pentland/Rueter 1994; Barrett I998; Feldman/Pentland 2003). Hargadon und Bechky (2006) definieren Probleme wie auch deren Lösungsfindung sowie die Generierung von Neuerungen als Routine. Sonenshein (20I6) geht gar so weit, dass er die Routine als Ort der Kreativität bezeichnet und nicht wie andere Ansätze die Arbeitsgruppe oder das Individuum. Variation, Neugestaltung bekannter Kombinationsformen sowie Fehler in der Reproduktion - oft Resultat routineartiger Aktivitätsprotokolle - begünstigen Kreativität (Chen 20I2: 63I ff.). Ortmann und Sydow (20I8) sprechen von der »tanzenden Organisation«, die in »selbst-geschmiedeten Ketten« kreativ handeln. Am Bei- 
spiel des Tanzes wird illustriert, wie eingespielte Handlungen die kreative Darstellung anleiten und die soziale Wahrnehmung lenken.

The dance event is an example of a situation where the consumers (i. e. the audience) of the dance music have conventional expectations, or a template, and the producers of the dance music (i. e. the dance musicians) follow routines, that allow them to perform so as to meet these expectations without much forethought or planning. (Ventresca/Kaghan 2008: 61)

Solche Handlungen reproduzieren und aktualisieren Routinen im Kontext von Improvisationen. Kreativitätsfördernde Praktiken verstehen sich darauf, Unbekanntes mit Bewährtem zu kombinieren und dadurch Erneuerungen anzuleiten. Diesem Dilemma begegnen vor allem Organisationen der Kreativindustrie, die sich schließlich einen Mittelweg zwischen Kreativsein und standardisierten Arbeitspraktiken suchen (Gilson u. a. 2005: 527). Diese Weiterentwicklungen des organisationalen Routinekonzepts distanzieren sich von Ansätzen, die Kreativität und Routine als kontradiktorische Konzepte festlegen, und verweisen auf die besonderen Kreativitätspotenziale. Entsprechend erschließt die vorliegende Arbeit Kreativität als Resultat routinierter und neukombinierter Tätigkeiten.

Folglich ist es keine Überraschung, dass sich auch in Modeunternehmen Aufgaben beobachten lassen, die über Tätigkeitsprogramme reguliert und standardisiert werden. So zum Beispiel die jährliche Inventur, die regelmäßige Auswertung statistischer Verkaufsdaten oder Bestellungen. Aber auch Prozesse wie die Kollektionsgestaltung, d. h. das Entwerfen von Schnitten und Stoffdrucken, deren konzeptuelle und technologische Umsetzung im Entwicklungsprozess, und die Praktiken der Selbstdarstellung in der Warenausstellung, den Fotoshootings oder den Modenschauen folgen musterartigen Anleitungen. Diese Prozesse werden von einem festgelegten Zeitplan reguliert, der sowohl die Fristen wie auch den Zeitrahmen dieser Aufgaben festhält. Viele Teilschritte nehmen aufeinander Bezug, bestimmte Sequenzen setzen andere Etappenziele voraus und werden so zur Grundlage für das weitere Vorgehen, und nochmals andere Teilaufgaben verlaufen parallel. Aus dem geteilten Handlungsrepertoire werden eingespielte Handlungsmuster situativ angepasst und individuell und als flexible Handlungsanleitungen interpretiert, die auch für unübliche Stimuli Anwendung finden. Routinen bilden die Grundlage für die rekursive Entwicklungslogik. Rekursivitäten begünstigen die Verbindungen innerhalb des Prozesses der Entwicklung neuer Kreationen und fördern die Bezugnahme über größere Zeiträume hinweg.

Ein Beispiel eines auf Routinen basierenden, sich rekursiv wiederholenden Vorgangs ist die Entwicklungsphase eines neuen Designs, auch Musterung 
genannt. ${ }^{44}$ Während dieser Phase nehmen die Akteure verstärkt Bezug auf vorhergehende Artefakte: Erst wird auf Skizzen und Beschreibungen eingegangen und daraus ein erster Prototyp hergestellt, dann wird dieser in einem oder mehreren Zyklen entwickelt, und schließlich entstehen daraus die ersten Musterkleider. Während bestimmte Abfolgen diesem Prozessschritt vorausgehen (Skizze entworfen, Kollektionsumfang bestimmt, Lieferanten aktiviert), entsteht stufenweise und rekursiv eine neue Kreation. Obwohl die Abläufe feststehen, bleiben sie in ihrer Ausführung variabel und können situativ angepasst werden - oder wie sich die Produktionsmanagerin bezüglich der Musterungen ausdrückt: »Also, es ist bei uns fast jede Saison ein bisschen anders, grundsätzlich [aber] ...« (Persönliche Korrespondenz, I2.0I.20I7). Während die Vorgehensweise immer wieder dem gleichen Schema folgt, kann die Reihenfolge der einzelnen Etappen variieren.

\subsubsection{Routinen als Handlungsgrammatiken}

Zunehmend verabschiedet sich die Organisationsforschung von alten, rigiden Deutungen routineartiger Tätigkeiten und an deren Stelle tritt ein flexibles, situatives Handlungskonzept, das lokale Deutungen und Translationen voraussetzt. Edwin Hutchins (I99I) illustriert anhand eines Beispiels einer navigierenden Brückenbesatzung auf hoher See, wie ein Schiff bei einer elektronischen Störung manuell navigiert wird, und hebt die spezifischen Kompetenzräume hervor. Diese Situation impliziert das Fehlen verlässlicher Handlungsmuster, weshalb die Crew das Problem nur im Team bewerkstelligen kann. Die Crew besteht aus Individuen mit je spezifischen Kompetenzen und unterschiedlichen Erfahrungen, die für die Aufgabe bereitgestellt werden und relevant für die kollektive, dezentralisierte Aufgabenstellung werden. Routine meint hier nicht das Arbeiten nach regelgebundenen Verfahrensanleitungen, sondern »distributed cognition«, das verteilte Erkenntnisvermögen, eine komplexe Aufgabe nur im Kollektiv lösen zu können. In der Doppeldeutigkeit zwischen Regelverfahren und situativem Handlungsanpassen liegt die jüngere Attraktivität des Routinekonzepts, weshalb sie in theoretischen Konzepten immer mehr hervorgehoben wird und der Routineforschung zu zunehmender Aufmerksamkeit verhilft.

Pentland und Rueters (1994) beschreiben die doppelte Bedeutung (Stabilität und Flexibilität) mittels des Prinzips einer Grammatik, die eine Syntax, einen Satzbau und Wörter beinhaltet, die beliebig zusammengestellt werden können respektive einem Set von Subroutinen und Routinen, die arrangiert

44 | Die ethnosemantischen Begriffe, die das Alltagswissen im Feld wiedergeben, werden in den Kapiteln zum empirischen Feldmaterial kursiv gekennzeichnet. Diese Hervorhebung beabsichtigt, den feldspezifischen Jargon ersichtlich zu machen. 
und zu neuen Routinen bzw. Prozessen zusammengefügt werden. Menschliches Organisieren hat wie jede Sprache eine Syntax, die Teil der Grammatik ist, den Bau des Satzes reguliert und die Verbindung von Wörtern zu Wortgruppen herstellt (Pentland/Rueter I994; Pentland I995)). Im Sinne von »more general set of structures « (ebd.: 543) beschäftigen sich Grammatiken mit der sprachlichen Form, den Gesetzmäßigkeiten und dem Bau einer Sprache. Die Grammatik schreibt kein spezifisches Resultat vor, sondern stellt lediglich ein Raster, eine Logik der Zusammenstellung zur Verfügung. Unabhängig von Raum und Zeit verwalten Grammatiken wie auch Routinen einzelne, ähnliche Ereignisse. Die Möglichkeiten werden an die aktuelle Situation angepasst und ermöglichen Flexibilität trotz Wiederholung erkennbarer Handlungsmuster (Feldman/Pentland 2003: 95). Die Grammatik bestimmt, welche Muster bestehen, kann aber keine Vorhersagen bezüglich individueller Kombinationsmöglichkeiten machen, denn "grammars describe a set of possible outcomes, not an individual outcome « (Pentland I995: 543). Mithilfe der Syntax können synonyme Teile einer Sequenz identifiziert und kategorisiert werden. Diese sind funktional gleich: »The meaning of the sentence changes, of course, but these forms are structurally equivalent« (ebd.: 545). Schließlich sind Wörter die kleinste sprachliche Einheit, die zu Wortgruppen geformt werden kann. Ihnen wird eine individuelle Lautung bzw. Bedeutung zuteil und sie sind mit den strukturellen Besonderheiten einer Situation verbunden; »they are constrained and enabled by the physical, ritual, and competence structure of the situation « (ebd.). Die kleinste Einheit, Bewegungen im Routinenansatz, Wörter in der Grammatik, werden von den Bedingungen und den Eigenheiten der Situation eingeschränkt. Sie sind teils von der physischen, rituellen und Kompetenzstruktur abhängig und werden teils durch sie aktiviert. Die Kleinteile lassen sich zu einem Lexikon zusammenfassen und können zu sinnhaften/sinnlosen oder möglichen/unmöglichen Verbindungen konstruiert werden. Das grammatikalische Raster definiert, welche Satzoptionen aus den unzähligen Wahlund Kombinationsmöglichkeiten einen Sinn beinhalten.

Von der Doppeldeutigkeit des grammatikalischen Routinekonzepts profitiert auch die Erklärung der Abläufe und Programme in der Entwicklung und Produktion neuer Moden. Die Modeindustrie sieht sich stets mit einer Vielzahl von Akteuren und somit mit unterschiedlichen Umwelten konfrontiert. Zudem hat sich das Modesystem um eine Sache formiert, die selbst Wandel und Vergänglichkeit verkörpert, nämlich Moden. Routiniertes Handeln begünstigt das Management der Entwicklung und Herstellung von Kleidern, rationalisiert die Marktinteressen und reduziert die resultierende Unsicherheit und Ambivalenz. Das Feldmaterial zeigt, dass Modeunternehmen vor allem in Bezug auf das Kerngeschäft und nicht trotz, sondern wegen des ständigen Wandels Verfahrensweisen und Handlungsmuster ausbauen, die zur internen Stabilisierung beitragen. Gewisse Vorgänge, die sich wiederholen, werden ab- 
sichtlich ähnlich - »so wie beim letzten Mal« - oder anders - »immer ein bisschen anders« - durchgeführt.

Auch Routinen in der Hervorbringung einer neuen Modekollektion können mithilfe des Prinzips der Handlungsgrammatik beschrieben werden. Die Gesetzmäßigkeiten der Modegrammatik sind ihre Zeitlichkeit, ihre Technologien sowie die Sicherheit der Erneuerung. Die organisationale Entwicklungsund Herstellungsweise einer neuen Kollektion kann in Anlehnung an die Grammatikmetapher als Syntax begriffen werden. Ähnlich der regulierenden Fähigkeit der Syntax, die den Satzbau steuert und Sätze aus Wörtern zusammenfügt, so regelt die Kollektion über die Zusammenstellung die einzelnen Modelle, indem sie die Verbindungsmöglichkeiten einzelner vestimentärer Details und textiler Elemente zu einem Modell und so die Modelle untereinander zu einer Kollektionseinheit verbindet. Die Kombinationsmöglichkeiten der Kleinstelemente, die textilen Details und Modelle, variieren von Kollektion zu Kollektion am stärksten, während die Kollektionszusammenstellung reguliert ist und dadurch relativ stabil bleibt. Die kleinsten, kreativen Einheiten in der Grammatik der Mode sind Labels und Etiketten, Schnitte, Farben, Fertigungsdetails, Fütterungen, Materialqualitäten wie auch Mengen, Größen oder Modellnamen. Die Baustruktur des Modells wiederholt sich im Zeitablauf und ändert den Inhalt, seine Zusammensetzung ändert sich aber kaum. Die materiellen Aspekte unterliegen kaum Veränderung, während die Bedeutung dieser von starkem Wandel betroffen ist. In den Creative Industries legen materielle, technische sowie funktionale Form- und Zweckgebundenheit den kulturellen Ausdruck fest (Moeran/Christensen 20I3; Rosso 20I4).

In den Modefirmen wird von der Arbeit nach einer Formel, also ein Jupe, ein Mantel, Blusen, Hosen etc. gesprochen, anhand derer abgewogen wird, ob die Kollektion durch einen weiteren Jupe oder eine zusätzliche Bluse erweitert werden soll. Ob ein weiteres Modell in die Kollektion aufgenommen wird oder nicht, hängt sowohl von der Syntax ab, d. h., ob es schon zu viele Jupes oder Blusen gibt, als auch von deren Kombinationsmöglichkeiten, d. h., ob es für den neuen Jupe/die neue Bluse auch ein passendes Oberteil/Unterteil in der Kollektion gäbe, und schließlich auch von ökonomischen Überlegungen, d. h., ob genügend Ressourcen (Finanzen, Zeit, Stoff) für Entwicklung und Produktion zur Verfügung stehen. Meistens scheitern solche Ergänzungsdiskussionen am finanziellen Budget der Kollektion. Um trotzdem einen gewissen Grad an Variation bieten zu können, ist das Abändern von Klassikern eine gängige Praxis. Durch diese saisonale Modifikation des Klassikers werden die Ökonomie wie auch die Kreativität bedient, und man variiert von Saison zu Saison mit der Interpretation des Modells. Das von einer Designerin im Interview genannte Beispiel beschreibt, wie diese saisonale Variation als »sportliche, beinahe Routinetätigkeit« beschrieben wird: 
Eh, nein, jetzt eben zum Beispiel Kaschmir-Schals habe ich vor zwei Wochen an einem Tag koloriert, ein Tag liegen gelassen, und die Seiden-Wollen-Schals habe ich noch nicht gemacht. Das werde ich in zirka zwei Wochen machen. Also das ist etwas, bei dem ich in etwa weiß, dass ich ungefähr drei Tage einrechne. Eh, und das ist repetitiv und (unv.) es ist etwas Klares. Also, ich meine, das ist wie, ich weiß auch nicht, Kaffee machen am Morgen, das kommt einfach zweimal im Jahr. [...] Und das ist auch ziemlich eine Routinesache, würde ich beinahe behaupten. Das ist wahrscheinlich der sportlichste Teil meiner Arbeit. Hingegen einen neuen Stoff zeichnen, einen Stoff neu zu kolorieren ist etwas, was mich länger beschäftigt und mir mehr Kopfschmerzen bereitet, aber eben auch Glücksgefühle (lacht).

Die Umgestaltung des Druckmusters bietet ein Beispiel dafür, wie mittels Routinen Erneuerung geschaffen wird.

Jones, Lorenzen und Sapsed (2015) argumentieren, dass sich die Sektoren der Creative Industries spezifisch anhand ihrer semiotischen Kodes und materiellen Basis als auch dem Tempo des Wandels (schnell/langsam) kategorisieren lassen. Die Mode zeichnet sich gemäß ihrem Schema durch eine konstante, materielle Basis aus, deren semiotische Bedeutung sich schnell und oft verändert. Das Problem der ständigen Kollektionserneuerung verlangt eine kollektionsindividuelle Lösung, wozu mögliche Themen und vorhandene Materialien stets von Neuem kombiniert werden, während die Kunstform, das Kleid, bestehen bleibt. Kombiniert man diese Idee mit dem Grammatikkonzept von Pentland und Rueter und erläutert sie am Beispiel der Modekreation, so erklärt die Handlungsgrammatik vor allem die materielle Entwicklung und anschließende Produktion, also die Zusammenstellung der einzelnen Modelle wie auch den Verbund zu einer einheitlichen Kollektion. Während die Materialen beschränkt sind, kann sich die semiotische Bedeutung dieser stark wandeln. Diese Idee erklärt jedoch nur einen Teil der Kreativitätshervorbringung in der Mode. Im Modesystem wird die materielle Basis zum Inbegriff von Verlässlichkeit, weshalb sich die Modefirmen Praktiken und Strategien zurechtgelegt haben, die Variationen ermöglichen. Obwohl das Material als Grundlage bestehen bleibt, kann es beliebig umgestaltet werden. Daraus entstehen Neuerungen. Dies hat den Effekt, dass bestehende Kollektionsmodelle inhaltlich-materiell stark verändert werden - oder um den Jargon, der oft in den Betrieben vorkommt, zu benutzen, werden die Modelle mittels Modellentwicklung und Modellanpassung (d.h. Ableitung, Verbesserung, Optimierung, Reduktion oder Variation) umgestaltet und neu ausgelegt. Die technologischen und organisatorischen Verfahrensweisen und Abfolgen dieser Prozesse aber ändern sich kaum.

Trotz minimaler Unterschiede werden die erneuerten Merkmale klar identifiziert und kommuniziert, jedoch kaum ein Vergleich mit Vorgängergenerationen hergestellt (Mora 2006: 339). Sieht man sich Modekollektionen erst 
einmal genauer an und vergleicht die Produkte über einen längeren Zeitraum, so lässt sich feststellen, dass sich Produkte innerhalb der Saisons nur minimal voneinander unterscheiden. Der Anschluss und die Orientierung an Tradition und Bewährtem ist eine weitere Eigenschaft von Neuerungen. Dennoch identifizieren Organisationen die Kollektion als eigenständige Einheit, die sich immer von Traditionen und Vorgängerkollektionen abhebt.

\subsubsection{Kreativität aus Bewährtem}

Die repetitiven Intervalle verstetigen sich zu einer richtungsweisenden und sicherheitsfördernden Routine, mithilfe derer der technologische Kern der Organisation ungehindert - aber angepasst - weiterarbeiten kann, ohne direkt von Umweltdynamiken tangiert zu werden. Einschränkungen wirken dann nicht als bedrohende Hindernisse, sondern als notwendige Bedingung für Kreativität (Rosso 20I4; Ortmann/Sydow 20I8). Die Organisation produziert die Neuerung auf der Basis verlässlicher und variationsfähiger Routinen:

Finally, by shaping familiar novelty, the organization regularly produces creative outcomes - novel behaviors upon which organizational members have collectively worked out the boundaries of - that nonetheless are familiar as BoutiqueCo [das Praxisbeispiel]. (Sonenshein 2016: 753)

Auf der Basis von Routinen wird Kreativität hervorgebracht und an Bekanntem und Vertrautem orientiert. Das Modesystem perpetuiert den Zyklus, wovon es selbst betroffen ist: während die Modefirmen der Dynamik des Modesystems unterliegen und dazu aufgefordert werden, regelmäßig Neuerungen zu generieren, perpetuieren sie das periodische, wiederholte Hervorbringen von modifizierten, erneuerten Inhalten selber. Es haben sich einige zuverlässige Verfahrensweisen herausgebildet, die hier nun als Neuheitsroutine bezeichnet werden. ${ }^{45}$ Die Beobachtung der Umwelt spielt im Kern eine nachgeordnete Rolle. Als organisatorisches Moment paradoxer Gleichzeitigkeiten fördert sie die Kombination von Vielfalt, Stabilität und Flexibilität der kombinierten Organisationsformen. Während sich die Organisationsformen kaum verändern, werden die Materialien spielerisch zu Neuerungen zusammengestellt. Gleichzeitig unterliegen die Materialien semiotischen Veränderungen. Als Folge davon reproduziert das Modesystem sowohl das Bekannte und Bewährte in Form stabiler Verfahrensweisen und Handlungsmuster, wie es auch Diversität und

45 | Kern (2006) spricht von "vectors for creativity", denn diese Regeln, worunter auch Routinen gefasst werden, sind richtungsweisend für das, was später als Kreativität wahrgenommen wird. 
Veränderung fördert, in dem es Rekombinationen und spielerisches Zusammenstellen veränderlicher Elemente zulässt.

Die Routinetheorie leistet einen wertvollen Beitrag zur Kombination von Stabilität und Flexibilität, Verlässlichkeit und Variation. Soziologisch lassen sich sowohl die Struktur der Organisation wie auch der Prozess des Organisierens mit Routinen erklären: »Routines occupy the crucial nexus between structure and action, between the organization as an object and organizing as a process « (Pentland/Rueter I994: 484). Die Routinetheorie eröffnet mögliche, theoretische Weiterführungen der Modekreationsprozesse in unterschiedliche Stoßrichtungen. Die strukturelle Unterscheidung von Stabilität und Wandel, Routine und Neuheit ist gemäß Reckwitz auf Weber und Marx zurückzuführen und erklärt die »doppelte Paradoxie des Neuen«: Erstens sind Organisationen mit Neuartigkeit und Unberechenbarkeit mit der Umwelt konfrontiert, weshalb sie die innerorganisationalen Strukturen standardisieren und rationalisieren (Weber). Folglich sind Routinen musterhafte Organisationsformen. Gleichzeitig aber, so die zweite Paradoxie, finden Neuerfindungen innerhalb der Organisationen in abgeschotteten Einheiten und Departementen statt, die getrennt von produktiven und administrativen Routinebereichen angelegt sind. Der Vorteil davon ist, dass die Organisation auf Dynamiken in der Umwelt reagieren kann, ohne die ganze Organisationsstruktur zu unterminieren (Marx). Demzufolge sind Routinen variable Bestandteile von Organisationsprozessen. Somit wird versucht, »technischen Wandel im Rahmen einer sachlichen Ordnung « zu unterhalten (Reckwitz 20I4: 138).

Solche Standardisierungsinteressen stehen in Zusammenhang mit industriell hergestellten Kreationen und erfolgen, um Gleichartigkeit und Verlässlichkeit zu gewähren und die wechselhaften Bedingungen der Umwelt zu bewältigen. Diese Darstellung basiert auf einer grundlegenden These der Organisationsforschung: die Trennung von Kernoperationen und peripheren Aktivitäten in der Organisation sowie die Beachtung der Kontingenzen aus Umwelt und deren Einfluss auf die Organisation. Schließlich sind Routinen notwendig, um (flexibel) organisieren zu können, genügen aber nicht, um ein vollständiges Bild der Organisation zu zeichnen (Obstfeld 20I2).

\section{2 Öffnung und Schliessung: Kontingenztheorie}

Die soziale Netzwerkanalyse und der Neoinstitutionalismus sind zwei der dominierenden Denkschulen der Organisationsforschung. Für die Analyse der Kreativität in der Modebranche bietet sich jedoch die Kontingenzforschung an. Der Mehrwert der Kontingenzforschung für die Kreativitätsforschung zeichnet sich sowohl an der empirisch auffälligen Technologiezentriertheit als auch am typischen Professionsbezug der Kreativwirtschaft ab. Im Jahr I967 fasst 
Thompson seine Vermutungen zu modernem Management zusammen und wagte einen Ausblick:

But in the modern societies, it appears, we have passed from the era in which control and coordination of technological activities were the central administrative challenge, into an era in which organizational rationality is the core of administration, and the administration of multiorganization projects and activities is the central challenge. (Thompson 2008: 158)

Die Verwaltung multiorganisationaler Projektaktivitäten ist insbesondere auch für die Kreativitätshervorbringung in der Modeindustrie wichtig geworden. Thompsons Kontingenzansatz hat seither nicht an Relevanz eingebüßt. Dieses Kapitel nimmt darauf Bezug.

Kreativität hat mit Technologien zu tun. Technologien sind wesentlicher Teil der Organisationsstruktur. Thompson (2008 [1967]) und Mintzberg (I989) verstehen die Technologie, gemeint sind Wissen genauso wie auch Apparaturen, als Schlüssel zur Organisationsstruktur, die sich aus der Gesamtheit aller Handlungen und Entscheidungen zusammensetzt und in Anpassung an situative Faktoren der Umwelt gestaltet wird. Technologien werden von Organisationen eingesetzt, um bestimmte Ziele zu erreichen. Demnach determinieren technologische Notwendigkeiten und Sachzwänge die Organisation und bedingen Entscheidungen, die zur Basis weiterer Kollektionsentwicklungen werden. Von der Technikforschung werden Technologien auch als »a set of pieces of knowledge both directly >practical< (related to concrete problems and devices) and >theoretical (but practically applicable although not necessarily already applied), know-how, methods, procedures, experience of successes and failure and also, of course, physical devices and equipment « bezeichnet (Dosi G. I982: I5I f.). Technologien sind im erweiterten Sinne auch Praktiken, Wissen sowie Erfahrung und somit für alle Organisationstypen wichtig. So gehören sie auch für die Creative Industries, und nicht weniger für die Hervorbringung von Neuerungen in der Modeindustrie, zu den zentralen Bestandteilen des Organisierens.

Das Feldmaterial zeigt, dass Herstellungstechniken - die Strickmaschine - und Produzentenbeziehungen - Partner und Freundschaften - zentrale Spezifika der Organisation sind. Aus diesem Grund können sie als der kontinuierliche Betrieb im operativen Kern (z. B. in Form von Klassikermodellen) wie auch als Methode der Umweltbeobachtung (z. B. in Form von thematischen oder stilistischen Anpassungen der Modelle) begriffen werden. So versuchen Organisationen, die technologische Tradition im operativen Kern zu wahren und sich gleichermaßen für neuartige Einflüsse in der Peripherie zu öffnen. Verschiedene Beispiele aus dem Feldmaterial illustrieren den Prozess der Anpassung der Designs an die Technologie. In der vorliegenden Analyse sind 
Technologien als maschinelle Techniken (einschließlich der Infrastruktur oder Apparaturen) sowie als Wissen und (handwerkliche) Fähigkeiten der Produzenten zur Hervorbringung einer neuen Modekollektion zu verstehen.

\subsubsection{Buffering - zum Schutz operativer Betriebsaktivitäten}

James D. Thompson baut seine These auf der gegenseitigen Abhängigkeit von Technologie und Umwelt auf, denn diese konstituieren die Hauptquellen der Unsicherheit für Organisationen. Folglich führen Änderungen in der Technologie und der Umwelt zu Änderungen in der Organisation. Nach Thompson besteht die technologische Rationalität aus einer ökonomischen und instrumentellen Dimension - mit ökonomisch meint er die Erreichung des Ziels durch Einsatz von »least necessary expenditure of resources«, während die instrumentelle für die generelle Erreichung des Ziels steht (Thompson 2008: I4 f.). Technologien sind seriell verflochten, zeichnen sich durch gleichartige Verfahrensweisen aus, entwickeln Folgeabhängigkeiten, deren Unregelmäßigkeiten mittels Repetition eliminiert werden und die mithilfe eines angereicherten Erfahrungsschatzes Störungen vorbeugen und Fehlschläge reduzieren. Anhand solcher Standardisierungsmaßnahmen wird der Betrieb kontinuierlich gepflegt.

Die ökonomischen, technischen und funktionalen Aktivitäten werden aufgrund ihrer Anbindung und Einbettung in die Umwelt maßgebend von dieser bedingt (ebd.: 19). Organisationen sind keine geschlossenen Systeme, sondern vielmehr von einer offenen Systemlogik geprägt. Die offene und organische Systemlogik der Organisation hat zur Folge, dass Organisationen von ihrer direkten Umwelt beeinflusst und gestört werden. Um ein Vordringen von Umwelteinflüssen in den operativen Kern zu verhindern und so zentrale Aktivitäten zu hindern, werden Organisationen dazu angehalten, den operativen Kern hermetisch abzuschotten und so Ambivalenzen und Kontingenzen zu kontrollieren. Schließlich ist ein »buffering « des Betriebskerns notwendig, weil die Instabilität der Umwelten die Organisation andauernd mit wechselnden Problemen konfrontiert (ebd.: $2 \circ$ f.). Um sich den Umwelteinflüssen nicht komplett zu entziehen, müssen Organisationen eine Balance zwischen betrieblicher Effizienz und der Sensibilität für Umweltveränderungen finden.

Umwelteinflüsse lassen sich gemäß Thompson in zwei Kategorien teilen: Diejenigen, die von der Organisation kontrolliert werden können, heißen »constraints«; diejenigen, die sich verstetigen und auf welche Organisationen keine Einwirkungsmöglichkeit haben, werden als »contingencies « bezeichnet (ebd.: 24). Dadurch werden die zentralen Technologien vor ambivalenten, kontingenten Umwelteinflüssen gepuffert. Als Folge werden Inputfaktoren, d. h. Maschinen, Apparaturen und Infrastruktur, einer regelmäßigen Wartung respektive Wissen einem Training unterzogen, sodass keine Überraschungen auftreten; 
Lagerhallen werden eingerichtet, die trotz diskontinuierlicher Nachfrage eine kontinuierliche Entwicklung und Produktion ermöglichen und Personal wird andauernd angeheuert und ausgebildet - unabhängig von der Nachfrage (ebd.: 20). ${ }^{46}$ Für die Output-Seite bedeutet das, dass man Inventuren macht, also dass bestehende Bestände berechnet und abgebaut werden. Das Durchführen von Outlets und Ausverkäufen - in der Mode beispielsweise schlecht verkaufte Saisonfarben oder Druckmuster - führt dazu, dass der Bestand reduziert und die Entwicklung und Produktion neuer Kreationen aufrechterhalten wird. Letztere Form des Pufferns ist vor allem für Organisationen, die Massenware herstellen, von Bedeutung. Die Identifizierung von Schemata und Mustern der Umweltdynamiken - bei Mintzbergs späterer Weiterentwicklung übernimmt das Management in Modeunternehmen die Aufgabe des »pattern recognizer« (Mintzberg I989: 2I7) - können Organisationen dabei helfen, Unterbrüche vorauszusehen und abzupassen (Thompson 2008: 22).

Mintzberg nennt drei Kooperationsmechanismen (Standardverfahren, z. B. Routinen; Planung, z. B. die Terminierung von Projekten; oder gegenseitige Anpassung, z. B. Reaktionen auf Modetrends), womit sich die Organisation Spielraum verschafft, um den Betrieb von der Umwelt respektive kurzfristige Trends von langfristigen Moden zu puffern (Thompson 2008: 22). Per Standardverfahren wird in der Organisation eine stabile Situation hergestellt, welche die kontinuierlichen operativen Aktivitäten trotz temporärer Störungen pflegt. Modefirmen richten eine kombinatorische Infrastruktur der Technologie und Kreativität ein, wodurch die Kreation von Neuerungen effizient und ökonomisch organisiert wird. Mittels Kooperationen wird der Dynamik der Umwelt begrenzter Einfluss eingeräumt, wodurch sich die Organisation ein Fenster zur Umwelt hin installiert. Schließlich muss die Modefirma stets neue, relevante Informationen berücksichtigen und sich gegebenenfalls anpassen. Dabei entgeht Thompson (wie auch Mintzberg) aber, dass genau aus den >profanen< Prozessen der Standardverfahren Veränderungen hervorgehen (Mintzberg I989: 218; Thompson 2008: 56).

Die Pufferung wird vor allem durch die lose Kopplung von Organisationseinheiten innerhalb der Organisation, aber auch zwischen der Organisation und ihrer Umwelt errichtet. Insofern handelt es sich bei der Kreativitätshervorbringung in Modeorganisationen weder um total entkoppelte Systeme, in denen alle Einheiten unabhängig voneinander operieren, noch um eng gekoppelte Systeme, denn dafür sind die Motive und Ansprüche der unterschiedlichen Projektmitglieder zu divers. Orton und Weick sprechen von lose gekoppelten Systemen und betonen damit die Gleichzeitigkeit der gegenseitigen Abhängigkeit und Eigenständigkeit:

46 | Thompson (2008: 20) erwähnt hier die Militärausbildung trotz Abwesenheit von Krieg. 
The fact that these elements are linked and preserve some degree of determinacy is captured by the word coupled in the phrase loosely coupled. The fact that these elements are also subject to spontaneous changes and preserve some degree of independence and indeterminacy is captured by the modifying word loosely. The resulting image is a system that is simultaneously open and closed, indeterminate and rational, spontaneous and deliberate. (Orton/Weick 1990: 204 f., Hervorh. im Orig.)

Als lose gekoppelte Systeme fällt es Organisationen leichter, die Umwelt zu beobachten und systemfremde Richtwerte und Vorgaben zu registrieren.

Je weiter man sich vom operativen Kern der Organisation entfernt, umso diversifizierter wird das Wissen (das sich zunehmend nicht mehr nur auf die Kerntechnologie beschränkt) und umso mehr Kommunikation ist erforderlich. Wie Mode hergestellt wird, muss selten neu bestimmt werden, und so erfordert die Produktion nach Verfahren weniger Kommunikation als die Planung eines (neuen) Kooperationsverhältnisses; und diese erfordert relativ weniger Kommunikation als die wechselseitige Adaptierung aktueller Moden (Thompson 2008: 56). Je komplexer die Sozialstruktur der Organisation und je dynamischer die Umwelt, desto dezentraler und zerstreuter die Entscheidungsfindung und umso wichtiger wird Kommunikation (ebd..: 73). Je weniger Macht und Wissen die Organisation über ihre Umwelt hat, umso mehr ist sie auf Vermittlerrollen (»boundary-spanning units«, in der Netzwerkforschung auch Broker oder Gatekeeper genannt, s. Kap. 5.I) angewiesen.

Die Theoriekonzepte zeigen sich auch im Feldmaterial. Die offene Systemlogik ist charakteristisch für die Creative Industries und Umweltbeobachtung findet in der Mode über Modemagazine, während Modewochen oder Modenschauen, sowie in geteilten Produktionsstätten statt. Nicht nur über diese Beobachtungsfenster, sondern auch über temporäre Kooperationen öffnen sich Modefirmen gegenüber der Umwelt, verschließen sich ihr aber auch, indem die Firmen Umwelteinflüsse - Inspirationsquellen, Ideen und Trends - nur gefiltert und kontrolliert inkorporieren. Die Modefirmen haben je eine spezifische Methode der Pufferung des Betriebskerns entwickelt: Generalisten und Spezialisten. Die generalistische Ausprägung zeigt sich darin, dass nicht nur Mode, sondern eine ganze Reihe verschiedener Produktekategorien entworfen und entwickelt werden. Damit kann der typische Zyklus der Modeindustrie umgangen und kontinuierlich produziert werden - sei es Kissenbezüge aus Seide, Stofftiere aus Baumwolle sowie Schmuck aus Silber oder Schals aus Kaschmir, Seide und Wolle. Diese Art der Fertigungstechnik wird von Kieser und Walgenbach (2010) mit »Werkstattfertigung« mit geringer Mechanisierung bezeichnet. Die Generalisten zeichnen sich durch eine geringe Stückzahl der Werkstattfertigung aus, die wiederum geringe Spezialisierung der einzelnen Teilbereiche bedeutet: 
Die einzelnen Stellen müssen mit einer Vielzahl unterschiedlicher Anforderungen fertigwerden; das verbietet eine weitgehende Aufspaltung der Verrichtungen. Die Unterschiedlichkeit der zu fertigenden Produkte schafft einen relativen hohen Koordinationsbedarf, erschwert aber eine detaillierte Vorausplanung der Fertigung. Die Koordination dürfte weitgehend den persönlichen Anweisungen der Meister und Vorarbeiter und/oder der Selbstbestimmung der Arbeiter überlassen bleiben. (Kieser 2014: 177)

Während der Werkstattcharakter des Generalisten einen niedrigen Spezialisierungsgrad aufweist, zeichnet sich die Fertigung nach dem Fließprinzip mit hoher Mechanisierung durch einen hohen Spezialisierungsgrad aus, weshalb die einzelnen Stellen der Organisation wenige Tätigkeiten verrichten (Kieser 20I4: I77). Die Planung wird in diesem Fall einerseits durch Programmierung, andererseits durch hohe Anforderungen an die Materialwirtschaft erreicht. Im Spezialist-Standort wird aufgrund des Spezialisierungsgrades eine schnelle und zeitlich beliebige Umsetzung ermöglicht. Innerhalb der Produktionsabteilungen besteht wenig Koordinationsbedarf, zwischen den einzelnen Projektschritten aber umso mehr. Diese wird durch die geografische Nähe zwischen Designabteilung und Produktionsstandort begünstigt. Beide Firmen produzieren Kleiderkollektionen im saisonalen Modezyklus, ergänzen diese jedoch mit einer Methode der antizyklischen Kreativitätsproduktion.

Ebenso zeigt sich die antizyklische Fertigung in der Reproduktion von Klassikern oder Evergreens (beliebte Kleidermodelle wie auch Accessoires), die stark gefördert und befürwortet werden, denn diese sind nicht nur technologiekonform, sondern initiieren auch geringes Maß an Veränderung. Diese Fortführung von Klassikern hat zur Folge, dass die Produktion (auch antizyklisch) unberührt fortgesetzt und das daraus generierte Wissen zu Angebot und Nachfrage verglichen werden kann.

So lehnen beide untersuchten Firmen ab, zu trendige oder modische (mödelige) Kleidung herzustellen, und bevorzugen demgegenüber hohe Qualitäten in den Materialien und der Verarbeitung dieser. Trends und Moden werden als $\mathrm{zu}$ unsicher und wenig lukrativ angesehen, weshalb die Investition in Klassiker und cash cows befürwortet wird. Die Selektion wird dann nicht anhand aktueller Trends und Moden gemacht, sondern an organisationalen, technischen wie auch personellen Bedingungen ausgerichtet, z. B. an der Strickmaschine. Trotz Neuerfindungen wird permanent eine Kollektionsbasis fortgesetzt, deren Kleidungsstücke wenig zusätzlichen Arbeitsaufwand benötigen (und erfahrungsgemäß viel Geld einbringen). Die Verkürzung des Entwicklungsprozesses konnte im Feld wiederholt beobachtet werden:

Evergreens sind Kleider, die schon lange im Sortiment sind und praktisch nichts mehr kosten, weil sie IT-technisch nicht weiterentwickelt werden müssen. Der Schnitt kann 
direkt in die Produktion gehen, ohne von jemandem abgenommen, überprüft oder kontrolliert zu werden.

Modelle werden aufgrund guter Verkaufszahlen und daraus folgend mehrjähriger Produktion zu Klassikern. Folglich leistet die Fertigung von Kleiderkollektionen einen Beitrag zur Traditionalisierung der Technologie im Unternehmen. ${ }^{47}$ Details werden dann angepasst, sodass das Modell dennoch von Kollektion zu Kollektion variiert.

Aus diesen Gründen hat die technologische Einschränkung bestimmte Anpassungen in der Umsetzung und Herstellung der Kollektion zur Folge. Die Modefirma ist stark abhängig von der Ausrüstung und dem Können der Hersteller respektive deren Infrastruktur. Die Technologie, hier eine Maschine, bestimmt die Umsetzung. Die Verhandlung der Machbarkeit wird auch im Interview mit dem Geschäftsführer als relevante Größe genannt:

In der Diskussion mit der Designerin werden dann die Farben, die Bindung mittels der Strickentwicklung und der Schnitt diskutiert. Da einigt man sich eigentlich vor allem auf die Möglichkeiten der Maschine. Dann heißt es: machbar oder nicht? Eine andere Frage gibt es nicht.

Die Machbarkeit, also die Technik, die Infrastruktur und die Maschine determinieren die Kollektionsinhalte. Die Bedingungslosigkeit der Technik hat ebenso Auswirkungen auf die Entwicklung und Produktion der Modekollektion, insbesondere in Belangen wie Farbwahl und Farbmuster. So erzählt die Designerin anhand der im Laden hängenden Oberteile, dass

[a]uch gerade bei der Farbwahl die Designerin gebunden ist: Da der Faden immer längs durchläuft, kann sie keine Muster produzieren lassen. Das ist auch der Grund, weshalb praktisch alles im Sortiment uni ist. Einzig kann sie eins-eins-Ringelmuster oder Mehrfärber machen lassen. Wobei der Geschäftsführer erwartet, dass sie sich die mehrfarbigen Kombinationen im Kopf vorstellen kann. Das ist aber eine große Herausforderung und sie hat auch schon mal eine Farbkombination gewählt, die nicht gepasst hat und sich schließlich nicht verkaufen ließ. Inzwischen weiß sie, dass die gewählten Farben Ton-in-Ton sein müssen, ansonsten lässt sich die Farbkombination nicht verkaufen.

47 | Diese Traditionalisierung der Modelle wird dann problematisch, wenn mehrere Jahre alte Exemplare zur Reparatur zurückgebracht werden, um den versprochenen service après vente, also die Reparatur, einzufordern. Aufgrund veränderter EU-Richtlinien wird dieses Modell nicht mehr auf diese Weise produziert, und die Modefirma sieht sich verpflichtet, die Unkosten der Reparatur zu tragen. 
Beide empirischen Beispiele erläutern das Konzept des Pufferns in der Entwicklung von neuen Kollektionsmodellen. Durch neue Entwürfe, Ideen und Modelle werden das Können und die Techniken der Maschine gewahrt. Insofern schließt die Organisation den Betriebskern vor disruptiven Alternativmöglichkeiten ab.

Auch das Verhältnis zu Produzenten wird in Thompsons Sinne als Technologie verstanden, denn das Wissen und die Erfahrung wie auch die Fähigkeiten der Produzenten sind zentral für die Aufrechterhaltung des operativen Betriebs. Die Organisationsmitglieder bezeichnen die Beziehungen mit den Herstellern aufgrund ihrer langjährigen Verbindung zum Modeunternehmen als Partnerships und manchmal sogar als Freundschaften. Diese Bezeichnungen verweisen auf eine intime und vertraute Beziehung zwischen den Akteuren. Ebenso sind damit die gegenseitige Zuverlässigkeit der Vergabe des Auftrags und seine Ausführung gemeint. Der CEO, der gleichzeitig auch die künstlerische Leitung innehat, führt im Interview aus:

Aber noch wichtiger ist, und das finde ich den zentralen Punkt [...] dass man verlässlich ist, wenn man mit Leuten arbeitet, wenn man Partnerships hat; also dass man diesen immer wieder ein Einkommen gibt und schaut: "Ok, das letzte Jahr konnten sie für 200.000 Franken liefern..." und wir müssen schauen, dass wir innen wieder für 200.000 Franken Ware abkaufen können, weil die eine Familie und Kinder haben, die eine Ausbildung brauchen.

Diese offene Reflexion über die selbstverständliche Freundschaft mit Handelspartnern ist ein prominentes Thema in dieser Firma. Daraus entsteht jedoch nicht nur eine wechselseitige Abhängigkeit, sondern auch eine Tradition der Zuverlässigkeit, indem den Interessen der Handels- und Produktionspartner stattgegeben wird. Die Designerin erinnert sich im Interview an eine Situation, welche die Wechselseitigkeit der Beziehung darstellt:

Also, noch ein weiteres Beispiel [...]; die Frauen unserer Sticker. Weil das [Sticken, Verzierungen] machen ja mehrheitlich Männer, also zu 99 \% Männer, muslimische Männer in Indien. Deren Frauen, das sind meistens einfach Hausfrauen, die aber handwerklich extrem begabt sind und mit diesen wollen wir nun häkeln. Und unser Partner in Indien: Er hat als unser Seidenproduzent angefangen und mittlerweile ist es wirklich unserer Partner und seine Tochter ist eine Freundin von mir. Und diese haben jetzt auch einen Laden von uns eröffnet. [...] Und er [der Produzent] ist inzwischen so sensibel, dass er sagt: "Hey! Es wäre cool, wenn ihr mal wieder was häkeln könntet. Hast du nicht gerade eine Idee? " Und das mache ich dann total gern, weil ich es schön finde, so ein bisschen "Doch, ich glaube, da kommt eine Idee..." Und dann haben sie was zu tun. Also ich finde das sehr wichtig. 
Die Bezeichnung Partnerschaft suggeriert folglich mehr als nur eine zuverlässige und langfristige Zusammenarbeit auf Vertrauensbasis; es handelt sich um handwerkliches, technisches Know-how, auf das die Modefirma angewiesen ist, weshalb sie diese Technologien abschottet und schützt. Aus operativen Gründen werden die Produktionsstätten rege bedient und können als Folge regelmäßige Aufträge verzeichnen. Diese Nähe und Loyalität und der regelmäßige Kontakt zu den Vertragspartnern vereinfachen direkte Kontrolle und Überwachung bei der Herstellung (Djelic/Ainamo 1999: 63I). Nicht selten wird deshalb auch auf den eigenen Vorteil verzichtet, um die zentralen Handelspartner beizubehalten. Ein Beispiel einer solchen Überlegung beschreibt die folgende Aufzeichnung der Gedanken, über welche die Designerin laut reflektiert:

Die Designerin wurde von der Tochter des Produzenten angefragt, ob sie den Restposten eines Stoffs für ein Kleid aufbrauchen darf. Die Tochter will "einzelne Stücke, die im Lager rumliegen « aufbrauchen. Doch die Designerin hat Bedenken, dass daraus ein Kleid entsteht, das nicht den Standards des Unternehmens entspricht. Sie überlegt sich die Folgeprobleme von einem Nein, die größer wären, als wenn sie den Stoff einfach freigibt. Sie wiegt die Folgen einer Absage ab und entschließt sich, der Bitte stattzugeben. Die Entscheidung wird von der möglichen Reaktion des Produzenten und der daraus entstehenden Stimmung zwischen dem Unternehmen und dem Partner beeinflusst.

Zum Schutz der Technologie werden die Interessen verschiedener Gruppen, hier des Handelspartners, verfolgt. Zu diesen Interessen zählen die gemeinsame Vergangenheit und das geteilte Ziel, Produktionen und Arbeitsplätze zu sichern. Diese werden zur verpflichtenden Bedingung und prägen die Zukunft beider Organisationen. Diese Beispiele zeigen, welchen Einfluss die Hersteller auf den Prozess der Kollektionsentwicklung, die technologische Umsetzung der kreativen Details, haben. Die Technologien bedingen die künstlerische Ausdrucksform in etlichen Facetten..$^{8}$ An die Stelle der schier unendlichen Auswahl des Machbaren tritt die Technologie als Leitprinzip.

Die Technologie determiniert auch organisationale Kognitionen, vor allem das individuelle Selbstverständnis und die Arbeitsaufgabe der Designer. Während einer Musterungssitzung wurden Hindernisse als Möglichkeiten beobachtet, deren Problematisierung von »anything goes« $z u$ »nothing but« wird:

48 | Die Farbwahl ist in beiden Unternehmen Aufgabe der kreativen Leitung. Jedoch hat die Maschine einen großen Einfluss darauf, wie die Farbe am Kleid schließlich zum Ausdruck kommt. Nicht selten gibt es Probleme mit der Einheitlichkeit der Farbbäder oder Farbkombinationen. Die Farbwahl wird nicht minder wie der Schnitt durch die Technologie bedingt. 
Teil der Aufgabe der Designerin ist der Umgang damit, dass es sich um "Strickware" handelt, also die Technik und das Material im Vornherein schon bekannt sind und den Entwurf einschränken. Weiter ist Teil der Aufgabe, die sich die Designerin bei jeder Kollektion von neuem stellt, dass die Entwicklung der Produkte wenig Aufwand für die Strickentwicklung geben soll. Je mehr Strickentwicklung, desto mehr Aufwand in der Programmierung. Für sie persönlich ist der Kreativitätsprozess aber erst abgeschlossen, wenn alles am neuen Kleidungsstück optimiert ist. Da der Produzent aus Kostengründen nur ein bis zwei Prototypen herstellt, muss sie versuchen, das Ziel der Optimierungen vorher zu erreichen.

Die Technologie wird als selbstverständlich betrachtet und in die Form eines Mottos (weniger ist mehr) oder einer Regel (etwas Biederes, etwas Vintage, etwas für Männer) eingearbeitet. Hier wird eine persönliche Karriereagenda formuliert, eine individuell formulierte Maxime zu verfolgen, die der allgemeinen, professionellen Agenda eines Creative Directors ähnlich sieht, auf einzigartige Weise das Unternehmen künstlerisch zu prägen. ${ }^{49}$ Diese Agenda drückt sich stilistisch in der Kollektion aus, wodurch die Organisation in einer Nische verortet werden kann, z. B. bei den new basics. Die professionelle Aufgabe wird langfristig verfolgt und verstetigt sich zu einem unerreichbaren Optimum, dessen Erlangung sukzessiv verfolgt wird. Folgende Beobachtungen stammen aus Musterungen:

49 | Die Maxime ist die ausformulierte Version der persönlich-individuellen Agenda der kreativen Karriere, die das höchste Ziel der eigenen Arbeit beschreibt. Demgegenüber ist es die professionelle Agenda des Creative Directors, im Unternehmen einen typischen, kreativen Stil zu etablieren. In beiden Unternehmen kommt es vor, dass die Grundlagen der Schnittentwicklungen auf Herrenschnitten aufbaut. Beim Spezialistenstandort lässt sich beobachten, dass die Reduktion bis zum Minimum - also der reduziertesten Form der schnitttechnischen, funktionalen und ästhetischen Bedingungen eines Kleidungsstücks - als oberstes Ziel genannt wird (reduzierte Schnitte). Die Eingrenzung und Optimierung findet anhand von Herrenschnitten, die für Damenkörper abgeändert werden, statt, um so die Schnörkel und das Unnötige zu umgehen. Beim Generalistenstandort wird eine ähnliche Idee aufgedeckt: Aufgrund des Wechsels in der kreativen Leitung des Unternehmens hat sich auch die Agenda verschoben. Die Tradition wurde mit einer neuen Ästhetik substituiert, was sich nicht nur in den Schnitten, Stoffdrucken oder der Farbwahl widerspeigelte, sondern auch das alte Logo zum Verschwinden zwang. Während diese Entwicklung bei den Kleiderkollektionen geduldet wurde, hat sich die Substitution des neuen Logos nicht bewährt, weshalb heute das alte und das neue Logo nebeneinander bestehen. 
Ein Pulli, der ziemlich groß ausfällt, wird es nicht in Größe L geben, dafür aber in XS. Die Designerin ergänzt und zeigt am Prototyp, dass dann das Ärmelloch etwas höher angesetzt werden muss, damit der Pulli gut sitzt.

Ein Modell, das in der Besprechung etwas mehr Aufmerksamkeit bekommt, ist z. B. die Schulter an einem Pulli. Es handelt sich um eine Mischung zwischen Raglan und Sattelschulter. Der Strickentwickler hat eine Mischung produziert, denn bei der technischen Erklärung stand etwas ungenau "Raglan-/Sattelschulter". Die Produktionsmanagerin kommentiert, dass man sich nun für die Sattelschulter entschieden hat. Die Designerin macht aber den Eindruck, dass sie lieber die Raglanschulter bekommen hätte. Sie entschließt sich, den Prototypen mit nach Hause nehmen und dort über die optimale Lösung nachzudenken.

Die Optimierung, die immer Inhalt der Mustersitzungen ist, findet in Absprache mit Experten statt, so z. B. dem Strickentwickler, dem Schnitttechniker oder dem Schneider. Diese ergänzen die geplante Veränderung mit dem technischen Fachwissen. Aus der kreativen Idee und der technologischen Umsetzung entsteht etwas Neues, nämlich ein Kompromiss, wie die Designerin an Verkaufsmodellen zeigt:

Ein anderes Beispiel sind die Löcher in einem Kollektionsmodell, das einem japanischen Textil-Design gleicht. Die Designerin hat Löcher verschiedener Größen entworfen. In der Produktion wurden alle Löcher auf die gleiche Größe vereinheitlicht. Das Resultat unterscheidet sich vom Originalentwurf. Dennoch wurde das Modell gut verkauft und hat sich über mehrere Saisons bewährt.

Optimierung wird zum Grundbegriff verschiedener Interessen und Ziele. Was für die Designer Optimierung am Kleid bedeutet (die Löcher in verschiedenen Größen zu stricken), heißt für die Techniker Aufwands- respektive Kostenoptimierung (alle Löcher auf die gleiche Größe zu vereinheitlichen).

Auf den ersten Blick wirkt dieser Vorgang als Filter für die technologische Machbarkeit der Idee. Versteht man diesen Vorgang als kontingenztheoretisches Problem, so wird die Prüfung der Neuerungen durch Techniker zur Kontrollinstanz zwischen Umwelt und Organisation, bei der der Grad der Störung eruiert wird. Ist die Abweichung von bestehenden Verfahrenstechniken zu groß, ist die Störung zu stark und darf deshalb nicht umgesetzt werden. Umgekehrt wird die Störung als nicht bedrohlich eingestuft, wenn die Idee in Anlehnung an bestehende Technologien produziert werden kann. Das temporäre Produkt, das Gegenmuster, wird dann nicht gegenüber der künstlerischen, ursprünglichen Idee gestellt, sondern gegen technologische, ökonomische und funktionale Machbarkeit und Passung geprüft. Ideenentwürfe, Modelle und Themen werden so der organisationalen Kontrolle unterzogen, sodass diese die 
Kernaktivitäten nicht fundamental tangieren. Vor diesem Hintergrund hängt ökonomischer Erfolg nicht vom Design, sondern vom technologischen »fit«ab.

Die Herausforderung liegt aber nicht nur in der Anpassung und Kontrolle an externe Einflüsse durch die Fokusorganisation. So reflektiert die Produktionsmanagerin während des Feldaufenthalts darüber, wie sich die Produzenten verhalten, wenn ihrerseits Abschottungsbemühungen zum Vorschein kommen.

Die Produktionsmanagerin bekommt von den Produzenten eine EMail [...], die beschreibt, was in der Sommerkollektion alles nicht machbar, also nicht "nähbar", ist. Sie bespricht sich mit der Schneiderin und der Designerin, denn technische Hinweise haben optische Effekte und deshalb muss die Antwort abgesprochen sein. Manchmal will der Hersteller auch schlicht keinen Aufwand für die gewünschte Version betreiben. Dann werden die Produzenten von der Modefirma darauf hingewiesen, dass es so und nicht anders gefertigt werden soll.

Aussagen wie nicht machbar oder bis jetzt ohne Erfolg bedeuten keine Rückschläge, sondern verweisen auf Abschottungsbemühungen seitens der Produktionspartner gegenüber den Auftraggebern. Gleichermaßen wie die Modeunternehmen ihren technologischen Kern abschotten, versuchen die Produktionspartner, den operativen Kern vor Umwelteinflüssen zu schließen. Vor allem Organisationen, die in eine dynamische und kurzlebige Umwelt eingebettet sind, sind bestrebt, die externen Umwelteinflüsse zu prüfen, sich abzuschotten und nur eventuell, partiell mit den innerbetrieblichen Technologien zu kalibrieren.

\subsection{2 "Adhocracy" - zur Möglichkeit rapider Anpassung}

Die Schilderungen über Entwicklungs- und Herstellungsformen der Creative Industries erinnern an organische Organisationsformen wie Henry Mintzbergs »Adhocracy« (I989) oder kognitive Gruppierungen, die sich um Praktiken oder Wissen formieren (Porac/Thomas/Baden-Fuller I989; Porac/ Thomas I990; Brown/Duguid I99I; Boland/Tankasi I995). Modefirmen sind innovative Organisationen, die nicht nur eine wandelbare Organisationsstruktur aufweisen und sich an die Umwelt anpassen, sondern auch traditionelle Apparaturen und Wissen wahren. Professionen spielen hierbei eine nicht zu unterschätzende Rolle.

Henry Mintzberg (I989: 98 ff.) skizziert eine Organisationskonfiguration, die auf Hervorbringung von Neurungen spezialisiert ist, und nennt sie »Adhocratie« (Abb. 2). Die Bezeichnung weist auf die Wandelbarkeit und Flexibilität der Strukturen hin, die schnellstmöglich auf Umwelteinflüsse reagieren und sich an diese anpassen können. 
Abb. 2: Die innovative Organisation »Adhocracy«

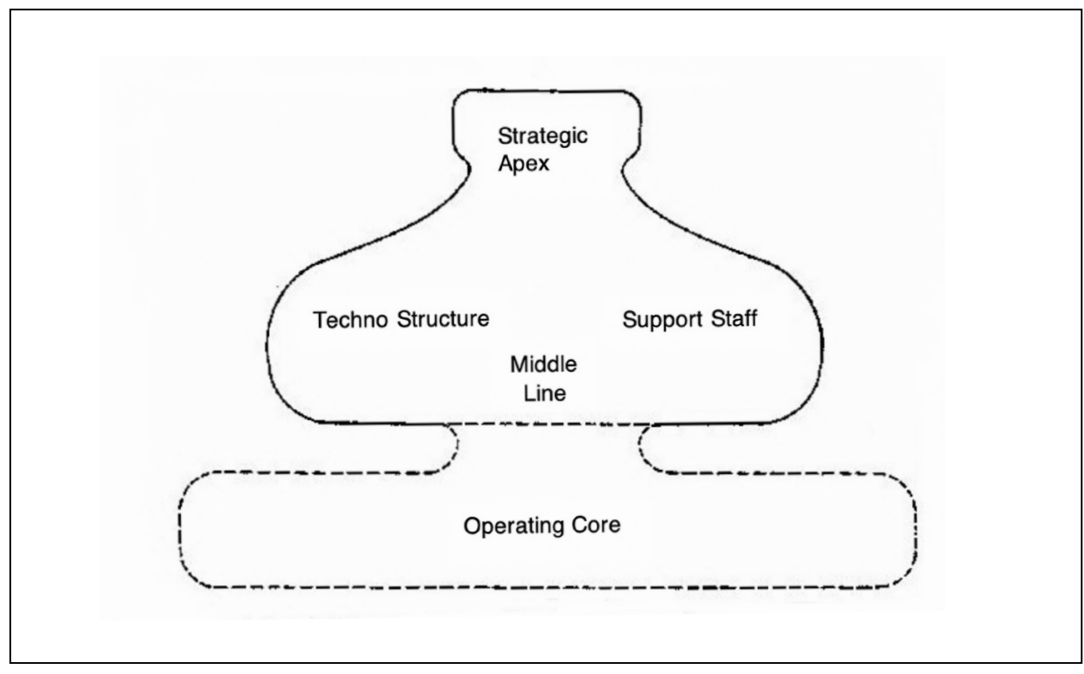

Quelle: Eigene Darstellung nach Mintzberg (1979: 198)

Die innovative Organisation ist eine Weiterentwicklung der Basisorganisation und besteht aus dem operativen Betriebskern (operative core), in dem die tatsächliche Produktionsarbeit geleistet wird - also aus den Produzenten und den Produktionsmaschinen -, und dem strategischen Management und Verwaltungszentrum (strategic apex), also sowohl der organisatorischen als auch der kreativen Leitung. Zusammen bilden der operative Kern, die strategische Spitze (Topmanagement) und der Mittelbau (middle line, mittleres Management und Verwaltung, d. h. permanente Organisationsmitglieder) die innere Koalition, die direkten Einfluss auf das Betriebsgeschehen im Kern der Organisation hat. Wird die Technostruktur (technostrucutre) der »Adhocracy« mit anderen Konfigurationen verglichen, so fällt auf, dass die Standardisierungen und Formalisierung, wie auch administrative Aufgabenbereiche (support staff, temporäre Projektmitglieder), und die unterstützenden Arbeitskräfte - damit ist zusätzliches, nicht operatives Personal gemeint - keine separaten Organisationseinheiten darstellen, sondern Bestandteil des mittleren Managements sind. Sowohl die administrativen Tätigkeiten als auch die effektive Projektarbeit in der Technostruktur und die geschäftsleitenden Aufgabenbereiche werden in der innovativen Organisation von einem arbeitsteiligen Körper durchgeführt, weshalb sie sich nicht kategorisch in verschiedene Einheiten aufteilen lassen (vgl. Kap. 6.3). Das Mitspracherecht und die kollektive Entscheidungsfindung in Projekten erklären, weshalb die strategische Spitze kaum vom mittleren Management unterschieden werden kann. Die kreative Leitung interagiert in Projekten mit unterschiedlichen Kooperationspartnern und konstituiert eine 
wechselhafte »dominante Koalition«, wodurch strategische Wahlmöglichkeiten und neue Entscheidungsgewalten entstehen und zur Anwendung kommen (Child 1972 und 1997). Insofern wird dadurch ein Argument gegen die vereinfachte Darstellung des funktionellen Imperativs »struktureller Bedürfnisse« entworfen (Child 1972: I4). Projektorganisationen bieten den Vorteil, dass sie die Dynamik zwischen Kontinuität und Wandel aufgreifen und sich dadurch einen strukturellen Vorteil verschaffen.

Innovative Organisationen bestehen aus einer selektiv dezentralisierten, organischen Sozialstruktur, die Verantwortlichkeiten und Recht auf Entscheidung und Mitsprache auf Subeinheiten verteilt und die auf flexiblen und informellen Kommunikations- und Entscheidungswegen basieren (Mintzberg I989: I9 6 ff.). Weiter setzt sich die Organisationskonfiguration aus Ad-hoc-Projektteams zusammen - deshalb auch die Bezeichnung »Adhocracy «-, die von Demokratie und kollektiven Arbeitsformen geprägt ist, denen bürokratische Verwaltungsstrukturen und Effizienzdenken nachgeordnet werden. Die Deutungshoheit bleibt jedoch auch bei Ad-hoc-Teams bei der kreativen Leitung. Die Besonderheit dieser Konfiguration ist einerseits das schnelle Reaktionsvermögen aufgrund selektiver Dezentralisierung und informeller Kommunikation als auch die Möglichkeit der Umweltbeobachtung über das extern rekrutierte, zusätzlich benötigte Spezialistentum. Die Koordination verläuft teilweise in, mehrheitlich über die gemeinschaftlichen Projekte, die vom Management koordiniert werden, weswegen es sich um eine Mischform gegenseitiger Anpassung und direkter Aufsicht handelt. Ebenso kann in den Creative Industries beobachtet werden, dass sowohl Normen bzw. institutionelle Erwartungen auf die Organisationsstruktur einwirken, als auch die Fähigkeiten standardisiert sind und deshalb Personal ad hoc und temporär angeheuert wird (ebd.: Ior ff.).

In der kreativen Organisation wird die Bedeutung des unterstützenden Personals hervorgehoben, denn die Führungskräfte und das Fachpersonal stehen in informellem, wechselseitigem Austausch mit den operativen Arbeiten. Der »support staff « ist losgelöst vom operativen Kern und kann direkt Einfluss auf das betriebliche Geschehen im Kern nehmen (ebd.: 98 ff.). Deshalb sind es die freien Mitarbeiter der Ad-hoc-Teams, welche die Entscheidungsfindung auf verschiedene Projekte verteilen. Die informelle Kommunikation koordiniert und dezentralisiert (Mintzberg spricht von »selective decentralization«) die sonst übliche formalisierte Hierarchie. In der innovativen Organisation befindet sich die relativ kleine »Technostructure« im operativen Kern, denn Adhocratien verwenden komplexe Produktionstechnologien, die im Kern betrieben werden (ebd.: 205). Umgeben ist der Organisationskomplex von der organisationalen Umwelt, der externen Koalition, die - ähnlich den Konzepten des organisationalen Feldes oder der Produktionsnetzwerke - aus Organisationsbesitzern, Mitarbeitern wie auch anderen Gruppen, z. B. Lieferanten, Partnern, Konkurrenz oder der Kundschaft, oder Regierungen, Behörden, Kommunen, 
Gewerkschaften, aus der allgemeinen Öffentlichkeit oder speziellen Interessensgruppen besteht (ebd.: Iо०).

Kreativität ist gemäß Mintzberg die Fähigkeit, auf Dynamik und den Wandel in der Umwelt einzugehen und mit daraus entstehenden intraorganisationalen Herausforderungen umgehen zu können. »To innovate means to break away from established patterns« (ebd.: I99). Damit weist Mintzberg auf die zentrale Eigenschaft der innovativen, kreativen Organisation hin: mit bestehenden Strukturen und Bedingungen zu brechen, mithilfe schneller Wahrnehmungsund Anpassungsfähigkeit spontan und rasch auf Wandel und Veränderung eingehen zu können, während der operative Kern kontinuierlich weiterproduziert. Die Umweltbeobachtung wird hier als eine der Hauptaufgaben der Organisation beschrieben, denn ihr entstammen nicht nur die zusätzlichen temporären Projektmitglieder, sondern auch gestaltungsrelevante Inhalte, welche die Technologien herausfordern. Die vorliegende Studie hebt insbesondere die Bedeutung von Technologien und Sprache für die Kreativitätshervorbringung hervor.

Vor diesem Hintergrund der spezifischen Schlüsselstellen, der Koordinationsmechanismen und der Verteilung der Entscheidungsmacht werden auch das Management und die Professionen in kreativen Sektoren beleuchtet. Laut Adhocratie besteht das Management aus professionell ausgebildeten und erfahrenen Experten, die nicht (ausschließlich) nach betriebswirtschaftlichen Kriterien leiten, sondern sich an »weichen« Kompetenzen wie Fähigkeit und Erfahrung orientieren. In dieser Hinsicht finden Entscheidungen, Kommunikation und Aufgabenverteilung qua Expertise, damit ist sowohl Fachwissen wie Erfahrung gemeint, und nicht durch Formalisierung, Autorität oder erweiterte Kontrollmechanismen und Sanktionen statt.

Dies trifft auch auf Designer bzw. Creative Directors zu. Es ist deshalb nicht weiter verwunderlich, dass es keine Ausbildung zum Creative Director gibt. ${ }^{\circ}$ Creative Director bezeichnet eine Position mit Erfahrung und Fähigkeiten, die der Funktion unabhängig von der Ausbildung zugeschrieben werden. Die schwache Professionalisierung in kreativen Organisationen zeigt sich darin, dass Kompetenz weder über Zertifikate noch Qualifizierungen ausgewiesen wird und auch formale Stellenbeschreibungen meistens fehlen. Infolgedessen dürfte es kaum überraschen, dass nicht alle Creative Directors formale ausgebildete Modedesigner sind. Von starker Professionalisierung der Position des Creative Directors kann dann gesprochen werden, wenn der Fokus auf die spezifischen Fähigkeiten gelegt wird, die exklusiv, aber nicht explizit sind. Bei-

50 | Ein Designer kann Mode studiert haben, aber nicht alle Modedesigner werden Creative Directors und nicht alle Creative Directors sind Modedesigner. Modedesign ist ein Studiengang, der an verschiedenen Hochschulen angeboten wird. Die Ausbildung wird zunehmend formalisiert. 
spiel dafür sind die Entscheidungshoheit bezüglich Themenfindung und die Ausarbeitung des Konzepts der neuen Kollektion. Allgemein lässt sich feststellen, dass die Professionen der Adhocratie bzw. der Modebranche hohe Freiheitsgrade in organisationalen Belangen sowie größere Gestaltungsmöglichkeiten ermöglichen und zulassen.

Da es den aufgabenzentrierten Projektformen der Adhocratie wie auch den Organisationen der Creative Industries an einem festgelegten Management fehlt, wendet sich das Aufgabengebiet der Designer zunehmend in Richtung eines handwerklich begabten Projektleiters. Die Folge ist eine kreative Leitung mit Doppelfunktion: Einerseits koordinieren sie die Organisation, andererseits führen sie mit unterschiedlichen Projektgruppen die Arbeit aus. Mintzberg beschreibt die kreative Aufgabe wie folgt:

The managers of adhocracy seldom 'manage in the usual sense of giving orders; instead, they spend a good deal of time acting in a liaison capacity, to coordinate the work laterally among the various teams and units. [...] So there must be a careful personal monitoring of projects to ensure that they are complement according to specifications, on schedule and within budget (or, more likely, not excessively late and not too far in excess of cost estimates). (1989: 200 und 206)

Diese Konfiguration ist deshalb die einzige organisch-dezentralisierte, die ihre Schwerpunktlegung vermehrt auf die Planung, Durchführung und Kontrolle von Ad-hoc-Projektteams statt deren Bürokratisierung legt. Aus diesem jedoch nicht ausschließlichen Grund bietet sich die Struktur für kreative Unternehmen an. In gleicher Weise wie Mintzbergs Auffassung der innovativen Organisation wird in organisationstheoretischen Aufsätzen der Kreativitätsforschung beschrieben, wie das Wegfallen des Managements oder formaler Hierarchien den kreativen Output der Projektteams fördert (Tschang 2007; Garud u. a. 20I3; Catmull/Wallace 20I4).

Trotz offiziellem Wegfallen der formalen Hierarchie und der Managementebene bewirkt die Professionalisierung eine zunehmende »Managementisierung«, die auch von Tschang bemerkt wird, dessen empirischer Schwerpunkt die Videospielindustrie ist: »Many lead designers act less as visionaries or conceptual idea generators and more as coordinators and negotiators with the team on a more rationally defined design, e.g., one that is designed to a publisher's requirements or someone else's vision" (Tschang 2007: 996). Die Rolle des Creative Directors verschiebt sich vom Visionär und konzeptuellen Entwickler hin zum Koordinator und Projektgruppenleiter. Die kreative Leitung übernimmt die Projektleitung, führt Verwaltungsaufgaben aus und koordiniert diese, während sie im direkten Austausch mit den Projektteams die Herstellung der Prototypen kontrolliert und die Umwelt auf der Suche nach inspirierender Veränderung und Aktualitäten beobachtet. Die Position des Creative Directors 
wird zu einer, die mehrere Aufgabengänge gleichzeitig erfüllen muss. Die organisatorische Einheit der Designabteilung und deren kreative Leitung sind mit der Kreativitätskoordination betraut, die als Ausschuss der Koordination und Kontrolle kreativer Arbeiten wirken. Entscheidungsbefugnisse werden dadurch dezentralisiert, wobei der Einfluss der Koordinatoren, des Designteams und des vorangestellten Creative Directors, auf deren (zugeschriebener) Fachkompetenz beruht (Kieser 20I4).

Gleichzeitig besetzt der Creative Director (häufig auch als Designer bezeichnet, womit die Grundfunktion dieser Position im Unternehmen festgelegt wird) die organisationale Rolle, die sich im Modebetrieb mit den kreativen Problemen auseinandersetzt. Solche Fragen sind Teil des Modealltags, denn die Branche legt viel Wert auf das kreative Werk (nicht die Kreativität der Tätigkeit!). Als Manager der Projektorganisation wird dem Creative Director eine kreative Kompetenz zugeschrieben, die in dieser Form einmalig ist: Weder innerhalb der Organisation noch in anderen Industrien gibt es eine vergleichbare Position. Damit sind bestimmte, zugeschriebene - und manchmal auch unzutreffende - Kompetenzen, Identitäten, Attitüden und Codes, ein bestimmter Jargon sowie ein spezifischer, aber nicht ausschließlicher Aufgabenkatalog gemeint. In der Form des Creative Directors hat sich Kreativität in der Organisation institutionalisiert. Von dieser Position aus wird die Kreativitätshervorbringung organisiert und koordiniert. Diese Institutionalisierung bedeutet, dass Kreativität organisational nicht an eine Person und deren Talent oder Begabung gebunden ist, sondern in Form der organisationalen Position und deren Rolle, kreative Probleme zu erfassen und auszuarbeiten, erscheint. Für die Organisationen ist es deshalb weniger relevant, wer diese Position besetzt, als dass sie besetzt wird.

Außerdem weist die dynamische, kreative Konfiguration einen hohen Grad an Flexibilität im Umgang mit externen Fachleuten aus: »Sophisticated innovation requires a very different configuration, one that is able to fuse experts drawn from different disciplines into functioning ad hoc project teams « (Mintzberg 1989: 199). Mintzberg spricht hier nicht nur die Koordination interdisziplinärer Zusammenarbeit an, sondern verweist auf Aad-hoc-Teams als fundamentale Einheit dieser Organisationskonfiguration (vgl. Kap. 6.3). Die künstlerische Leitung, das Management, verliert die Einbindung in die Entwicklungsprozesse und übernimmt die Funktion des Übersetzens eigener und fremder Ideen in eine gemeinsame (objektivierte) Vision und Projektsprache. Das Team (support staff) wird technisch und fachlich mehr eingebunden und erhält mehr Führungsmacht und Mitspracherecht. Schließlich koordinieren und kontrollieren die Ad-hoc-Teams mit den Projekten die ideengetreue Umsetzung der entworfenen Skizzen gemäß dem Kollektionsthema.

Ein solches Thema zu entwickeln und textil umzusetzen, ist weiter Aufgabe der kreativen Leitung, denn dieses markiert das konzeptuelle Zentrum 
der Kollektion. Bei der Nachfrage im Feld, wie Thema und kreative Arbeit zusammenhängen, erläutert die Designerin,

dass sie ein Thema hat und dieses dann den Schnitt definiert und dann ist das Tüfteln eigentlich vor allem noch dieses Thema als Stil durch die ganze Kollektion zu ziehen und kenntlich zu machen.

Das Thema als sichtbarer Stil in die Kollektion einzubauen, wird als Hauptaufgabe des Creative Directors genannt und ist somit eines der zentralen Probleme des Designprozesses. Als konzeptuelle Kategorie wird es für jede Kollektion neu entworfen und kann trotzdem angepasst werden. Gleichzeitig wird damit ein eigener, unverkennbarer Stil erarbeitet. Schließlich ist es die Aufgabe des Teams, die Umsetzung dieses Themas zu koordinieren (vgl. Kap. 7.2.I).

Die Inspirationen, die der Kollektionsentwicklung zugrunde liegen, stammen meist aus der Umwelt und müssen deshalb an organisationale Bedingungen, u. a. die Technologie, angepasst werden. Das führt zur organisationalen Abschottung des Kerns durch Experten und Techniken, ermöglicht gleichzeitig aber die Öffnung hin zur Anpassung an Umweltdynamiken. Die neuen Ideen aus der Umwelt tangieren die zentrale Technologie nicht, denn das Thema wird zwar frei gewählt und bleibt variabel, die textile Umsetzung dessen wird aber in Anlehnung an bestehende Apparaturen wie den Strickcomputer und vorhandenes Wissen der Partner durchgeführt.

Weiter sind auch die Farbwahl, Druckmuster oder textiltechnische Abänderungen und Zusätze als Neuerungen von stilprägenden Elementen zu deuten. Mittels Übersetzung in die Kerntechnologie können sie, obwohl sie neu und noch unpassend sind, von der Organisation übernommen und in die eigene »Handschrift« übersetzt respektive in die Technologie überführt werden. Das Übersetzen von Inspirationsoriginalen aus dem Feld der Mode in den eigenen Stil bedarf der künstlerisch-kreativen Expertise. In den Creative Industries und insbesondere in designaffinen Sektoren wie der Modeindustrie findet diese Übersetzung im Designprozess statt. Das direkte Kopieren von inspirierenden Originalen ist problematisch, weil es die künstlerische Integrität der kreativen Leitung respektive das Urheberrecht der Originalproduzenten untergräbt. Vielmehr wird die fremde Sprache als Störung aus der Umwelt gedeutet, die die Tradition des organisationalen Stils unterbricht, der als zentraler Bestandteil der unternehmerischen Selbstdarstellung aber auch der individuellen Kollektion gilt. Bei einem Besuch im Atelier erklärt die Designerin, wie sie gestalterische Inspirationen abändert:

Die Designerin will nichts in Südasien produzieren lassen, das ursprünglich nicht aus Südasien kommt. Das Original muss also übersetzt werden und diesen Zwischenschritt 
nimmt sie vor. Man würde es dem Produkt sonst ansehen, dass es eine nicht-authentische Kopie einer traditionellen Sache ist.

Anhand des Designprozesses werden die Originalideen aus der kulturellen und institutionellen Umwelt mittels Designs in die technologische Nachbildung übersetzt und wirken als betriebssicherndes Kontrollinstrument. Das Design macht möglich, dass sowohl Einflüsse aus der Umwelt in die Organisation einfließen und trotzdem die Kernoperationen aufrechterhalten werden können.

\subsubsection{Kreativität aus Kompromissen}

Einerseits schotten Modefirmen das Kerngeschäft ab und schließen die Grenze hin zur Umwelt, um ihren Einfluss dieser auf den Kernbetrieb zu beschränken. Andererseits öffnen sie sich und gewähren Inspirationen, Modellideen und Kollektionsthemen kurzfristig und partiell Einlass. Daraus entstehen Spannungen zwischen Organisation und Umwelt, die im Feld der Mode mit Kompromissentscheidungen bewältigt werden. Mintzbergs »grassroot model« veranschaulicht diese natürliche Spannung wie folgt: Anhand der Metapher des Unkrauts im Garten erläutert er, wie Unkraut ohne gehegt und gepflegt zu werden, wächst und sich vermehrt, verbreitet und anpasst, andere Pflanzen verdrängt, gedeiht und Ernte abwirft und schließlich wie sich der Samen erst in der Erde zu einer vollwertigen Pflanze entwickeln kann. Sowohl der Samen als auch die Erde tragen zur Fruchtbarkeit und erfolgreichen Produktion bei. Ebenso kann eine Idee erst in der Organisationsstruktur und durch die Technologie zur vollständigen, neuen Kollektion hervortreten. Einerseits muss dabei das Vergangene reproduziert, anderseits unterbrochen werden, um Neuerungen zu ermöglichen. Manchmal muss auf neu entstehende Muster eingegangen werden und diese müssen ins organisationale Repertoire im Kern aufgenommen, d. h. alte mit neuen Mustern ersetzt oder ergänzt werden (Mintzberg I989: 215). Die technologische Orientierung reduziert Kontingenzen, und Einschränkungen wirken somit unsicherheitsreduzierend. Ortmann und Sydow verweisen auf die von dem Philosophen Jon Elster als »Furcht der Freiheit« bezeichnete Vielfalt der sich eröffnenden Möglichkeiten: »Contingency can be considered as a form of freedom or as a threat, the latter because it creates an ever labouring mountain of possibilities that can paralyse actors Elster's >fear of freedom« « (20I8: 9I3). Technologie versteht sich somit als Kompromiss zwischen Kontinuität und Kontingenz, bezugnehmend darauf, dass Technik und Kreativität in Hinblick auf angestrebte Ziele stete Kalibrierung einfordern (Kieser 20I4: I83).

Diese Metapher veranschaulicht, wie die Spannung zwischen Technologiebewahrung und Umweltneugierde dazu führt, dass Kompromisse Bestandteil 
des Kreationsprozesses werden, die im Aussehen, beispielsweise Muster, und der Beschaffenheit, beispielsweise Elastizität, von der beabsichtigten Ideenskizze abweichen. Die Designerinnen rekapitulieren:

Auch in der Dreidimensionalität der Muster ist sie eingeschränkt: Während Zopfmuster bei handgestrickten Pullis sehr beliebt sind, hat es der Strickentwickler bis jetzt nicht geschafft, ein solches Muster zu programmieren. Kompromisse sind deshalb Teil des Überarbeitungsprozesses. Oft sehen Kompromisslösungen anders als gewünscht aus und verleihen dem Modell eine andere Elastizität.

[...] so lange über eine unbeliebte Art und Weise der Verarbeitung gesprochen wird, bis die Hersteller dann raten, ganz von der Ursprungsidee abzukommen und es auf ihre Weise machen wollen. Man muss abwägen und überlegen, wie wichtig die ursprüngliche Idee war und sonst davon abkommen und sich auf einen Kompromiss mit den Herstellern einlassen.

Kompromisse sind Hinweise darauf, dass die Kernoperationen und die Neuerungen in Konflikt miteinander stehen, dessen Lösung sich aus einer Modifikation oder Ergänzung der Technologie sowie der Überarbeitung des kreativen Designs ergibt. Während dieses Kalibrierungsprozesses werden entweder die technologischen Operationen bestätigt und verfestigt - dabei wird das Kleidermodell kaum verändert und nur marginal angepasst - oder auf die Umweltleinflüsse und Störungen reagiert und Veränderung akzeptiert - dafür werden die Kollektionsmodelle technologisch neu entwickelt.

An dieser Schnittstelle wirkt das Designteam als erste Schleuse, technologische Experten anhand von Schnitt oder der Machbarkeit auf den Maschinen als zweite Schleuse: Ein Thema wird als Idee oder Inspiration aus der Umwelt gefiltert, die kreative Leitung überprüft es und übersetzt es in die technische Sprache des Betriebs. Bevor der Entwurf in Produktion geht, wird er den Technikern zur Kontrolle vorgelegt und eine technologische Passung vorgenommen. Das Ziel ist die serielle Produktion kreativer Güter, die vorgängig bekannt ist und während des Entwicklungsprozesses berücksichtigt wird. In einer Mustersitzung wird kommentiert:

"Die erste Musterung sieht immer anders aus als die zweite, weil bei der zweiten oftmals schon bedacht wird, dass diese Teile in Masse produziert werden sollen".

Diese Einigung zwischen Technologie und Design findet mit Prototypen in Musterungen statt. In Mustersitzungen werden Prototypen im Designteam detailliert und einzeln besprochen. Im fortgeschrittenen Musterungsprozess, einem rekursiven Hin und Her zwischen künstlerischen Wünschen und kommerziellen Ansprüchen, spitzt sich die kreativ-technologische Verhandlung 
zu. Während die Designer Neuerung priorisieren und Diskontinuität wünschen, gewichten die technologischen Experten die Kontinuität der operativen Aktivitäten des Kerngeschäfts. Das Zweischleusenmodell veranschaulicht, wie Organisationen sich vor den Unsicherheiten aus der Umwelt schützen und interne Aktivitäten stabilisieren. Insofern steht es exemplarisch für die Ausdifferenzierung des »Grenzstellenmanagements « in der Organisation.

Modefirmen produzieren Kleiderkollektionen im Saisonrhythmus. Als Relikt der ehemaligen Orientierung an Jahreszeiten hat das Modesystem einen Zyklus institutionalisiert, der als globaler Standard die Modereproduktion dirigiert. Diese Periodizität hat institutionalisierte und standardisierte Verfahrensformen hervorgebracht, welche die Produktion und die Dynamiken der Umwelt entkoppeln. Das empirische Material hat gezeigt, wie Modeunternehmen versuchen, aufgrund der Beschleunigung dieser Reproduktion (zu den Hauptjahreszeiten Herbst/Winter und Frühjahr/Sommer kommen nun immer mehr Zwischenkollektionen) die Kernoperationen zunehmend von der Umwelt zu puffern. Diese partielle Öffnung und Schließung zeigt sich in den Produktionsverfahren und der Regulierung der Umwelteinflüsse über zwei Schleusen. Mehrere Akteure fungieren in diesem Prozess als Kontrollinstanzen. Hingegen wird die Öffnung über Kollektionsinhalte geregelt, die sich in Form eines Kollektionsthemas oder als stilistische Modifikation manifestieren. Über Kollektionen wie auch Kooperationen öffnet sich der Modebetrieb hin zur Peripherie, wobei die zentralen Technologien den Grad des Einflusses festlegen.

Für die Diskussion der Creative Industries und die Analyse von Modefirmen ist das Organisationsverständnis der Kontingenztheorie als Grundlage für weitere organisationstheoretische Diskussionen besonders ergebnisreich. Einerseits betont sie die Wichtigkeit und den Mechanismus der losen Kopplung und des »Bufferings « zwischen der betriebsrelevanten Technologie und dem zugehörigen Modesystem. Andererseits wird die strukturelle Beziehung zwischen Betriebskern und der Umwelt angesprochen.

\subsection{Permanenz und Temporalität: Projektforschung}

Wie weiter oben ausgeführt, befinden sich die Sektoren der Creative Industries und nicht zuletzt die Modeindustrie im Spannungsfeld zwischen Schutz und Öffnung und Variation und Verlässlichkeit. Das Feld der Mode ist weiter ein Beispiel für die Verknüpfung von permanenten und temporären Strukturen zur Hervorbringung von Kreativität, weil es zusätzlich das Spannungsfeld der Organisation zwischen Permanenz und Zeithaftigkeit dokumentiert. Die organischen Strukturen und Prozesse der Adhocratie schaffen insbesondere Raum für projektbasierte Koordinationsformen. Deshalb haben sich Projekte als zentrale Organisationsform im Modesystem herausgebildet. In dieser Form 
gleichen sie den von Obstfeld vorgeschlagenen »creative projects«, die zwar auf etablierten Handlungsweisen wie Routinen die Vergangenheit reproduzieren, zugleich aber die mögliche Zukunft vergegenwärtigen und sowohl Routinen wie auch das Unternehmen verändern. Er bezeichnet das kreative Projekt

as a form of interdependent action, conceptually distinct form, but closely related to, both stable and more adaptive depictions of routines. Creative projects are exploratory ventures that offer one means by which organizations and their routines change. (Obstfeld 2012)

In dem Projekt besteht die Möglichkeit des explorativen Entdeckens und Neugestaltens, weshalb Projekte in Kreativprozessen eine passende Ergänzung zur Routinearbeit sind. Als Bestandteil des kreativen Projekts fungiert die Knappheit von Ressourcen (Zeit, Personal, Finanzierung) nicht hindernd, sondern stimulierend (Rosso 20I4).

In Projekten spielt Temporalität in zweifacher Hinsicht eine bedeutende Rolle: Einerseits existieren Moden auf Zeit - sind ephemer -, andererseits sind die Ausarbeitung und Herstellung von Moden als Termingeschäft angelegt. Für die Analyse des Datenmaterials sind deshalb zwei unterschiedliche Ebenen von Projekten auffällig: Sowohl die Kollektion kann als Projekteinheit gefasst werden, die zeitlich begrenzt besteht und sich saisonal wiederholt und die Zusammenarbeit verschiedener Experten voraussetzt (die Moden), als auch deren einzelne Etappenziele und Sequenzierungen, die in ihrer Gesamtheit Kreativität hervorbringen, können als Projekte bezeichnet werden (die Tätigkeiten von Spezialisten). Für beide Ebenen von Projekten sind permanente und temporäre Strukturen und Organisationsformen nötig. Während die Permanenz der (formalen) Organisation vor allem durch routineartiges Handeln, aber auch in der organisationalen Infrastruktur (Hauptgebäude, Apparaturen, unbefristete Arbeitsverträge) reproduziert wird, sind es die Subprojekte, die das temporäre und wechselhafte Organisieren konstituieren.

Permanent organizing is about the establishment of more stable and persistent units and groups that have a long-term view and are continuously concerned with fostering creativity, providing regular feedback to creative solutions, as well as supporting the implementation of creative outcomes and the realization of their impact. Temporary organizing concerns the organizational capacity to initiate, conclude, or dissolve fluid and temporary initiatives that pursue creative outcomes and impact, such as creative projects and semiformal organizations. (Slavich/Svejenova 2016: 246)

Die Hervorbringung kreativer Güter bedingt die situative Balance zwischen permanenten und temporären Organisationsstrukturen. Die vorliegende Ar- 
beit erklärt die Verflechtung der Dualität von Permanenz, Zeithaftigkeit und Kreativität.

Dieses Kapitel nimmt Bezug auf den Diskurs zur Forschung temporärer und projektbasierter Organisationen und stellt direkte Verknüpfungen zur Kreativitätsforschung her. Dabei wird die Wiederholung als charakteristisches Merkmal der Kreationsprozesse herausgearbeitet und auf zwei Subthemen der aktuellen Projektforschung vertieft eingegangen: erstens die Projektaufgabe, deren Befristung und Wiederaufnahme; zweitens das Projektteam, dessen Konstellation, Vertrauen und Sprache, Wissenstransfer sowie konfligierende Zielsetzungen. Schließlich ist die Kollektion - und insofern auch die Kreativitätshervorbringung - das Resultat kommunikativer und organisationaler Kompromisse.

Mit ihrer Theorie der temporären Organisation haben Rolf A. Lundin und Anders Söderholm (I995) die Diskussion der nicht permanenten Organisationsformen lanciert. Die Autoren argumentieren, dass die Zunahmen von spezialisierten, problemzentrierten Task Forces, Programmkomitees, oder Aktionsgruppen zu erhöhtem Interesse an Projektorganisationen und ebendieser Forschung geführt hat. Die Problemzentrierung wird zum Hauptmerkmal der Gruppenformation. Die Aspekte Zeit (Horizont und Begrenzung), Aufgabe (einmalig oder wiederholt) und Team (Individuen als Ressource) spielen dabei eine zentrale Rolle. Das erklärte Ziel ist eine Situationsveränderung, in welcher mit dem Projekt eine Transition von einem Zustand in einen anderen erreicht wird. Diese vier Merkmale werden zum Hauptunterscheidungsmerkmal von permanenten und temporären Organisationsformen: »Permanent organizations are more naturally defined by goals (rather than tasks), survival (rather than time), working organizations (rather than team) and production processes and continual development (rather than transition) «(Lundin/Söderholm I995: 439). Lundins und Söderholms Konzeptualisierung fügt sich daher in das Mintzberg-Verständnis der innovativen Organisation bzw. der Adhocratie ein.

Temporäre Organisationsformen bevorzugen den Aufgabenauftrag gegenüber dem Zielfokus; Zeit- statt Überlebensbewusstsein; ein Team anstelle eines funktionierenden Verwaltungsapparats und ein diskontinuierlicher Übergang von einem Zustand in den nächsten ersetzt das Streben nach kontinuierlichem Fortschritt. Lundins und Söderholms Überlegungen haben sich als anschlussfähig erwiesen, und so hat sich in den letzten zwei Jahrzehnten ein Forschungsbereich etabliert, der sich nicht nur unterschiedlichen Ansätzen temporärer Organisationsformen widmet, sondern auch andere Themen wie beispielsweise Vertrauen, Kooperation, Wissen und neuere Konzeptualisierungen von Zeitlichkeit angeht.

$\mathrm{Zu}$ Beginn wurde vor allem von projektbasierten Organisationen gesprochen (DeFillippi/Arthur I998; Hobday 2000; DeFillippi 200I). Hobday de- 
finiert Projekte und erläutert dabei auch das Verhältnis zur projektbasierten Organisation.

[...] a project can be defined as any activity with a defined set of resources, goals, and time limit (e.g., for information technology or new materials), within a PBO [project-based organization] the project is the primary business mechanism for coordinating and integrating all the main business functions of the firm (e.g., production, $R \& D$, engineering, NPD, marketing, personnel, and finance). (2000: 875)

Projekte sind ressourcen-, ziel- und zeitbasierte Aktivitätsräume, die in der projektbasierten Organisation zur Hauptaktivität der Verwaltung und Geschäftstätigkeiten werden. Hobday kontrastiert Projekte gegenüber der Massenproduktion respektive Routinen, denn der Vorteil von Projekten, so sein Argument, sind vor allem »non-routine activites and complex tasks« (ebd.). Diese Grenze ist jedoch nicht trennscharf, wie sich in den vorliegenden, empirischen Beispielen zeigen wird.

Diese Art und Weise des Organisierens ist besonders geeignet für die Hervorbringung von Kreativität im Feld der Mode, in dem sie verbreitete Anwendung findet. Modeunternehmen koordinieren sowohl die Permanenz der Organisationseinheit als auch die Kurzlebigkeit von Projekten. Damit haben sie ein duales Modell des repetitiven Kollektionsprozesses geschaffen. Die projektspezifische Aufgabe unterscheidet sich nur wenig von Mal zu Mal: Die Kollektionsprozesse orientieren sich an einem repetitiven Handlungsmuster - wie Kollektionen gestaltet werden, bleibt in etwa gleich -, jedoch konzertiert sich jede Kollektion um ein neues Thema - was kreiert wird, unterscheidet sich. In diesem Sinn handelt es sich bei Unternehmungen in Modebetrieben nicht um alleinstehende, einmalige Vorhaben, sondern um eine integrierte, auf (unterschiedlichen Typen von) Projekten basierende Organisationsform.

\subsubsection{Economy of repetition - Mode aus Wiederholung}

Aus Projekten entstehen Projektumwelten (Grabher 2001, 2002 und 2004b) oder Projektnetzwerke (Sydow/Staber 2002; Jones/Lichtenstein 2009). Aufsätze, die Projekte als zentrale Organisationsform beschreiben, beziehen sich inhaltlich und empirisch stark auf die Creative Industries oder die Kreativität. Die Projektforschung schöpft aus der Forschung zu temporären Organisationsformen und der Kreativitätsforschung und generiert dadurch einschlägige Beiträge (Obstfeld 20I2; DeFillippi 20I5).

Zunehmend wird auf das Versäumnis hingewiesen, permanente und temporäre Organisationen in ein Konzept zu fassen (Engwall 2003; Grabher 2004b; Sydow/Lindkvist/DeFillippi 2004; Bakker 2010). Die konzeptuellen Ausarbeitungen erreichen ihren Höhepunkt Ende 2004, als in »Organization 
Studies« eine Spezialausgabe zu »project-based organizations« veröffentlicht wird. Bis dahin heben die Konzepte vor allem die kontextuelle Einbettung hervor - Engwall schreibt »No Project is an Island« (2003). Das Konzept »project-based organization « entwickelt sich nun in zwei Richtungen weiter: Das Interesse an Repetitionsformen temporärer Organisationsmodi ist gestiegen (Brady/Davies 2004; Schwab/Miner 2008). Diese Diskussion setzt die Einbettung und den Kontext einer permanenten Organisation voraus, auf welcher »economies of repetition« aufbauen können (Davies/Brady 2000). Gleichzeitig wird die taxonomische Beschäftigung mit Konzepten des temporären Organisierens wieder vermehrt aufgegriffen (Bechky 2006; Bakker u. a. 20I6). Bakker verweist auf die Brisanz und Relevanz des Problems, denn temporäre Organisationsformen sind zwar nicht neu, aber neue Organisationsformen sind oft temporär (Bakker 2oio: 466).

Wie oben erwähnt, sind temporäre Organisationsformen auch im Feld der Mode eine häufige Erscheinung. Das projektbasierte Koordinieren, welches das temporär eingestellte Personal, eine spezifische und festgelegte Aufgabe und einen temporären Horizont beinhalten, ist gerade für Kreationsprozesse in der Mode typisch. Modefirmen sind Organisationshybride aus permanenten und temporären Formen und changieren zwischen (un-)regelmäßiger Wiederholung und einmaliger Zusammenarbeit. In Modefirmen ergänzen sich administrative Verwaltungsaufgaben und künstlerische Arbeit. Folglich sind Modefirmen auch kommerziell-kreative Hybride, die ständig versuchen, Betriebsaktivitäten als Kernoperationen zu bewältigen, während sich die Organisation hin zur Peripherie öffnet und projektbasierte Kooperationen mit Spezialisten durchführt.

Projektbasierte Organisationsformen integrieren diverse Ressourcen, werden als schnelle und flexible Betriebsweise der Wissensverwaltung bezeichnet und bringen so den Vorteil mit sich, traditionelle Innovationsschranken $\mathrm{zu}$ umgehen. Hobday listet unter den Vorteilen der projektbasierten Organisation beispielsweise »managing increasing product complexity, fast changing markets, cross-functional business expertise, customer-focused innovation and market, and technological uncertainty« (Hobday 2000: 87I), und Sydow und Staber machen deren wissenschaftliche Prominenz an der Fähigkeit fest, »[to] circumvent traditional barriers to organizational change and innovation, since each project is presented as a temporary, relatively short-lived, phenomenon « (Sydow/Lindkvist/DeFillippi 2004: I475). Die projektbasierte Organisationsform ermöglicht eine rasche Reaktion auf dynamische Umwelten, erlaubt die Verteilung komplexer Arbeitsprozesse auf pragmatische Aufgabenbereiche und adressiert typische Ambivalenzen der Konsumentenmärkte, während die Form als wissenschaftliches Konstrukt einen Beitrag zu organisationalem Wandel und Innovation macht. 
Entstehen über wiederholte Projektarbeit Folgeabhängigkeiten, werden diese zu den Unterscheidungsmerkmalen von interorganisationalen, alleinstehenden Projekten. »Inter-organizational projects coordinate activities only for the lifespan of the project, which may extend five days or twenty years, whereas project-based organizing focuses on a durable organizational entity that uses projects to create its services and/or products « (Jones/Lichtenstein 2008: 235). Während Projekte über mehrere Jahre andauern können, grenzen Jones und Lichtenstein projektbasierte Formen davon ab und argumentieren, dass Projekte als Zweck zur Erlangung übergeordneter Aufgaben eingesetzt werden. Diese Aufgaben sind kleinere Projekteinheiten, z. B. Etappenziele und Arbeitssequenzen, die in ihrer Gesamtheit das Projekt neue Kollektion erstellen. Werden Projekte regelmäßig wiederholt, so lassen sich Kombinationsformen von permanenter und temporärer Organisation beobachten, so zum Beispiel die Einbettung der Projekte in bestehende formale Strukturen oder die Reduktion von möglichen Koordinationsproblemen, die vor allem bei heterogenen Teamkonstellationen auftreten (Lundin/Söderholm I995; Brady/Davies 2004). Diese Einbettung kurzfristiger Projekte in dauerhafte Unternehmensstrukturen charakterisiert die Kreation von Neuerungen in der Mode.

Die Einbettung temporärer Formen in permanente Strukturen spricht einerseits die Aufgabe, aber auch die Organisationsmitglieder an: während die Aufgabe innerhalb der permanenten Organisation repetiert wird, sind die temporären Projektmitglieder in »Mutterorganisationen« beheimatet. Projektbasiertes Organisieren umfasst schließlich sowohl das Vorhaben der Aufgaben und Aufträge als auch die Karrieren des Personals, des Teams und des Projekts (DeFillippi/Arthur 1998). Temporäre Organisationsformen unterbrechen die Permanenz des Organisierens und installieren Momente des Wandels und der Erneuerung. Die Mischung von Projekt- und Formalstruktur erleichtert das Entstehen von Vertrauen. Wiederholte Interaktionen ermöglichen die Entstehung von Reputation und Karriere, lassen aber dennoch Emergenz und Befremdung zu (Sahlin-Andersson/Linderoth 2002). Durch das Wiederholen von ähnlichen Vorhaben kann eine organisationale Identität konstruiert und aufrechterhalten werden. Darüber wird wiederum eine Marktnische bedient und es werden Verständnis, Wiedererkennungswert und Legitimität generiert. Diese marktwirtschaftlichen Vorteile können alleinstehende Projekte nicht leisten.

Die wiederholte Durchführung ähnlicher (Sub-)Projekte führt zwangsweise zu deren Einbettung in eine permanente Struktur und diese zu dem, was Davies und Brady (2000: 952) als »economies of repetition« bezeichnen:

Economies of repetition refer to the reductions in costs and improvements in project effectiveness gained by moving from the first-of-its-kind bid or project to the execut- 
ion of many similar types of bids or projects within cost, schedule and the required specifications.

Projektbasierte Organisationsformen sind besonders kostengünstig, denn sie bewirtschaften ihre Ressourcen auf reversible Weise und besitzen keine Fixkosten. Deshalb lassen sie viel Freiraum für Experimente (Sydow/Staber 2002). Grabher entwickelt dieses Konzept weiter und spricht mit den »economies of recombination« (2004a: IIO) dem Konzept etwas mehr Flexibilität und Varietät zu. Durch die wachsende Sammlung an Erfahrung und Wissen durch Herausforderungen, Bricolage, Kombinationen, glücklichen Zufall und Imitation entstehen Organisationsformen, welche die Kreativität und Produktivität aus der Wiederholung und Rekombination generieren und als Speicher für organisationales Lernen und organisationalen Erfolg fungieren. Durch Modifikationen sind die Creative Industries imstande, Neuerungen zu kreieren: Folgeprodukte schließen an Vorgänger an. Deshalb ist die Gleichzeitigkeit von Veränderung und Stabilität ein zentrales Merkmal der Creative Industries (Caves 20I2: 4).

Die Wiederholung von Projekten stabilisiert die Organisation, begünstigt Erwartbarkeit und Uniformität und erhöht die Voraussagbarkeit der Ergebnisse (Engwall 2003: 793 ff.). Rationalisierungstendenzen, also die zunehmende Standardisierung und Formalisierung der Tätigkeitsbereiche, Praktiken wie auch der Projektmitglieder, sollten mit überraschungsfördernden und routinebrechenden Maßnahmen ausbalanciert werden, um Kreativität und Neuheit auch in wiederholten Projekten zuzulassen (ebd.: 629). Während radikal neue Projektvorhaben exploratives Vorgehen begünstigen, kann die Anpassung eines Projekts von ehemaligen Erfahrungen profitieren. Engwall (ebd.: 8o2) spricht die Spannung an, in der sich auch Modeunternehmen befinden: Einerseits soll Unvorhersehbarkeit in Form von Kreativität durch neue, innovative Aufgabenstellungen initiiert werden, andererseits muss das Projekt an vorangehenden ausgerichtet werden, denn nur so kann die Organisation die Herstellung planen. Modefirmen sind daher gut beraten, permanente Strukturen mit temporären Formen zu ergänzen. Schließlich entsteht Mode im Spannungsverhältnis von dauerhaften Unternehmenszielen und temporären Aufgaben, wirtschaftlichem Überleben und künstlerischem Ausdruck, Organisationsverwaltung und Projektkoordination, Prozessoptimierung und Produkterneuerung. Diese Ambivalenzen werden in Projekten zusammengeführt.

Ein wichtiges Merkmal ist die Befristung des Projektbestehens: Projekte werden gegründet, um ein Vorhaben zu erledigen, das vorab zeitlich und inhaltlich festgelegt und mitgeteilt wird. Ein erhöhtes Zeitbewusstsein in Projekten ist die Folge (Bakker u. a. 2013). Durch die Festlegung des Vorhabens wird das Projekt ins Leben gerufen; mit der Erreichung der zeitlichen Frist und der Vollendung der Aufgabe löst es sich wieder auf. Die Befristung des Vorhabens, d. h. begrenzte Bearbeitungszeiten und verbindliche Abgabetermine, ist 
eine Bedingung der Modeherstellung. Auf der Organisationsebene zeigt sich die Zeitlichkeit in der täglichen Arbeit der Mitglieder von Modeunternehmen: Temporäre Aufgaben werden an Abgabeterminen festgemacht und schließen mit einer offiziellen Übergabe des aus dem Arbeitsschritt resultierenden Produkts ab. Abgabetermine (deadlines) werden im Kollektiv besprochen und festgelegt und gelten als zentrale Information, ohne die eine Aufgabe nicht erledigt werden kann.$^{5 \mathrm{I}}$

Sowohl Etappenziele wie auch Teilprozesse und Aufgabensequenzen werden in Projekten erledigt. Dabei bestimmt die Aufgabe sowohl die Zeitdauer als auch die Transition. Die Vollendung einer Teilaufgabe wird von einer Transition begleitet, die im kleinen Rahmen in Form von Übergaben stattfindet, die oft im Sitzungsformat gehalten werden und der Überprüfung und Kontrolle der Teilaufgabe dienen. Im größeren Umfang einer kompletten Kollektion kann eine solche Transition in Modezeitschriften oder auf Modenschauen inszeniert werden. Die Übergabe von Artefakten (Skizzen, Prototypen, Mustern) wiederholt sich regelmäßig nach einem abgeschlossenen, ausgelagerten Etappenziel und findet an der Schnittstelle zwischen zwei Prozessphasen (beispielsweise Designentwurf und Vorstellung dessen; Übergabe der entwickelten Prototypen an Designer) bzw. Projektsequenzen statt. Das zeremonielle Präsentieren konzentriert sich auf die Transition, die das Resultat der Projektaufgabe fixiert und hervorhebt. Im Interview erläutert die Produktionsmanagerin Folgendes:

Wir besuchen die Produzenten, kurz bevor sie mit der Produktion der Musterkollektion für die Modenschau beginnen. Und da wird dann wirklich zeitweise nochmals um die Details gekämpft. Vor Ort treffen wird die Leiterin des Büros, die Chef-Schneiderin und die Chef-Schnittmacherin und eine Person, die eine Zwischenstelle innehat. In der Schneiderei sprechen sie nur Ungarisch, aber die Frau vom Büro kann Deutsch und sie übersetzt dann gegenseitig. Und dann gehen wir Modell für Modell durch. Sie schauen das Modell an, nehmen es auseinander und kommentieren es. Manchmal sagen sie: "Oh, nein! Das kann man so nicht umsetzen! Wieso wollt ihr es so? Wir würden es anders machen. Kann man es vielleicht nicht andersrum legen?" Und dann wird verhandelt. Sie fühlen sich einer sehr klassischen Schneiderkunst verpflichtet, also richtiger Couture, wie es sich gehört. Beispielsweise ist Overlock, das einfache Absteppen des Saums, der Graus für sie. Das machen die Billighersteller, sagen sie. Und wenn wir so etwas in der Kollektion haben, natürlich als bewusster Look, z. B. an

51 | Auf institutioneller Ebene kennzeichnet die Taktung des Saisonrhythmus das spezifische Zeitbewusstsein des Modesystems. So vereinigt das Feld der Mode zirkuläre und lineare Zeitkonzepte: während Mode nie aufhört, sich stets weiterentwickelt und in Zyklen wiederkommt, repetiert und erneuert sich die Modeproduktion in linearen, vorab definierten Zeiteinheiten, die von einem Beginn, dem Kollektionsstart, und einem Ende, der Ablösung durch die nächste Kollektion, gerahmt wird. 
einem Kleid, wollen wir damit die starke Anlehnung an Couture runterbrechen. Solche Details müssen wir dann durchsetzen.

Dieses Beispiel der Übergabe aller Rohzutaten beim Produzenten zur ersten Prototypisierungsrunde markiert eine mögliche Transition. Die Veränderung des Entwicklungszustands eines Kleidungsstücks steht dabei im Mittelpunkt. Im Designteam werden einzelne Veränderungen an den Prototypen angesprochen, die im Mittelpunkt der Übergabe stehen, da an ihnen die Transition sichtbar wird. In diesem Sinne sind Übergaben selbstdefiniert und unumgänglich und folgen einem repetierten, unhinterfragten Handlungsritus. Folgende Feldbeobachtungen sind Beispiele für dieses iterative Vorgehen:

Die Designerin zieht jedes Muster an und kommentiert, was ihr dazu auffällt. Die Assistentin kommentiert die technische Korrektheit und Umsetzung des Musters. Die Produktionsmanagerin komplettiert diese Angaben mit der Richtigkeit der Stoffqualität, dem Vergleich zu ehemaligen Mustern und möglichen Lösungen, die damals im Bezug zu ähnlichen Problemen gefunden wurden und ihrer persönlichen Meinung.

In der letzten Musterung geht die Designerin alle Musterteile nochmals durch und gibt der Schneiderin an, was falsch ist und inwiefern es für die Show oder die Produktion geändert werden muss. [...] Die Schneiderin protokolliert Probleme wie falsche Fadenfarbe, Label-Kontrolle, Aufhänger, Ärmellänge, Knopfwahl, Größen-Anpassungen oder Abänderungen.

Durch die Repetition der Musterungen ist das standardisiert und systematisiert. Die Designerin geht dabei Prototyp für Prototyp durch, kommentiert den Schnitt in Relation zu Vorgängerschnitten, betont abweichende Details, nimmt Bezug auf die Materialqualität und macht Trag- und Kombinationsvorschläge. In (Gegen-)Musterung werden die Prototypenversionen besprochen und die Interpretationen der visuellen und verbalen Ausführungen abgeglichen und gegengeprüft. Probleme wie Verarbeitungsfehler, mangelhafte Qualität oder Unverständlichkeiten werden erst durch den Prototyp sichtbar. Daraus entsteht die Musterkollektion, die das Resultat eines rekursiven Selektionsprozesses ist, der sich mit der Farbauswahl, Stoffqualitäten, Schnitten und der Kollektionszusammensetzung beschäftigt.

Nicht nur Musterungen, auch andere Sitzungen, die spontan als Updates oder in regelmäßigem, oft wöchentlichem Turnus gehalten werden, zählen als permanente Organisationsformen. Oft werden sie zu Betreffen wie Einkauf, Weihnachten als verkaufsstärkste Zeit des Jahres, Inventar und Musterung festgelegt. Durch die Wiederholung sind sie Teil der permanenten Verwaltungsstruktur, zu welcher sie in Form von generierten Einkaufszahlen, Angaben zu Lagerbeständen und Protokollen aller Art beitragen. 
Die Arbeitsteilung und die Komplexität der Prozesse verlangt, dass die permanenten Organisationsmitglieder mit temporär angeheuerten Experten ergänzt werden. Mit der Beendigung des Vorhabens, im Fall der Mode entweder nach der Erledigung des Arbeitsauftrags (schnitttechnisches Muster herstellen, Stoffdrucke drucken, Prototypen fertigen etc.) oder nach der Veröffentlichung der Kollektion, ist die Projektaufgabe abgeschlossen und das Projektteam trennt sich. Das Projekt hört - temporär - auf zu existieren. Aufgrund der dauerhaften Strukturen der Organisation führt die Vollendung der Projektaufgabe nicht zum Zerfall dieser, sondern trennt permanente und temporäre Vorhaben und Karrieren. In latenter Weise existiert das Projekt aber weiter; einerseits im Netzwerk, aus dem die Mitglieder des Projektteams rekrutiert werden, und andererseits in der Wiederholung, die ehemalige Projektmitglieder erneut zusammenführt und ihnen eine neue, zu erledigende Arbeit in Aussicht stellt. Dadurch, dass sich Projekte über mehrere Generationen repetieren, verfestigt sich das beständige, latente Produktionsnetzwerk in einen Rekrutierungspool temporärer Akteure. Die befristete Wiederholung sequenzieller Tätigkeiten ist die typische Organisationsform und das zentrale Merkmal von permanent-temporären Projekten in der Mode. Kreativität ist ein Termingeschäft.

\subsubsection{Das Projektteam als Motley Crew}

Projektarbeit beinhaltet oft flexible Aufträge und Kurzarbeit, weshalb diese besonders vorteilhaft für Industrien sind, die Güter herstellen, die sich durch ihre Einmaligkeit und Expressivität von anderen Industrien und Gütern abgrenzen und einen großen Teil an Fertigkeiten und Intuition verlangen. Stinchcombe (1959) bezieht sich bei der Beschreibung von Projektarbeit auf das Baugewerbe (die »craft industries «), denn dieses illustriert die Zusammenarbeit verschiedener Handwerksbetriebe. Sinnbildlich für die Verschiedenheit der Projektmitglieder wird im Zusammenhang mit der Kreativindustrie von »motley crews« (Moeran 2009; Moeran/Christensen 2013) gesprochen, die zur Bearbeitung eines konkreten Problems und einer bestimmten Aufgabe rekruiert werden. Die Begriffswahl »bunter Haufen« spricht auf die heterogene Akteurskonstellation in Projektteams an und impliziert die Zusammenarbeit von permanenten Organisations- und temporären Projektmitgliedern. Auf diese Weise sind »motley crews« dem ähnlich, was Lindkvist (2005) als »collectivities « bezeichnet, und unterscheiden sich aufgrund des heterogenen Vokabulars, divergierenden Zielsetzungen und den unterschiedlichen Interpretationsweisen und Vorstellungen von Brown und Duguid (I99I) Darstellung der »communities of practice $\ll$.

Normative Angleichung durch Professionalisierung resultiert einerseits in der Vergleichbarkeit und der Austauschbarkeit von Personal und führt so zu 
Personalfluktuation, andererseits aber auch in einen hohen Spezialisierungsgrad und begünstigt damit Differenzierungseffekte (DiMaggio/Powell I983; DiMaggio I99I)..$^{2}$ Der Wissenstransfer und die Anreicherung von Erfahrung sowie das Entwickeln gemeinsamer Sinnbestände in Projektteams stehen im Zentrum des theoretischen Interesses und stellen insbesondere für projektbasierte Organisationen eine Herausforderung dar. Zugunsten des Projekts wird ein »changing mosaic of lateral project teams« (Boland/Tankasi I995: 366) zusammengestellt und dadurch versucht, die permanente Struktur der Routine mit variablen Formen zu ergänzen. Das »cycling and re-cycling « (Bakker 2ого: 475) der Projektmitglieder und Kooperationspartner wird durch Kurzzeitverträge verstärkt, wobei der Vorteil für die permanente Organisation darin liegt, temporäre Mitarbeiter schnell und reibungslos wieder abzustoßen, um dadurch finanzielle Fixkosten und externen Einfluss auf den Betrieb zu unterbinden. Mit jeder Projektbeendung wird der Ressourcenfluss unterbrochen: wenn ein Mitglied die Gruppe verlässt, leiden beide Parteien an einem Wissensverlust, weil kein organisationales Gedächtnis besteht. Das Mitglied verliert den Anschluss an bestehendes kollektives Wissen, und die Gruppe verliert die Expertise des Mitglieds (Fiol 2005).

Projektbasierte Organisationen verringern solche Probleme. Die Wiederholung von Projekten und deren Einbettung in beständige Organisationsstrukturen geht zwei grundlegende Probleme systematisch an: Durch Wiederholung können in Projektteams stabile Beziehungen entstehen, weshalb sich das Vertrauen von einer flüchtigen Basis in beständige Formen entfalten kann (Meyerson/Weick/Kramer 2006; Saunders/Ahuja 2006). Während in den Projekten die Mitgliederkonstellationen von Veränderungen gekennzeichnet sind, bauen die Projekteorganisationen auf einem stabilen und beständigen, loyalen Ressourcen- und Kooperationsnetzwerk auf. Aus vorhandenen, informellen Beziehungen entsteht eine Struktur formaler Zusammenarbeit. Die Folge ist weniger eine Serie bilateraler Beziehungen, denn eine von Freundschaft, Status und Ansehen geprägte Form des repetitiven Austauschs (Powell/

52 | Typisch für die Arbeitsdifferenzierung sind unterschiedliche Interessenslagen, Trajektorien der Berufskarrieren wie auch das Interesse an der Inszenierung der eigenen Tätigkeit. Nicht zuletzt für die Creative Industries mögen diese Merkmale zentraler sein und ausgeprägter erscheinen als in anderen Industrien, denn symptomatisch für diese Industrien ist nicht nur die individuelle (künstlerische) Selbstdarstellung, sondern auch die Relevanz der Projekterfahrung und sozialen Vernetzung. Trotz Unterschieden in der Professionalisierung, ist diese Auseinandersetzung nicht Gegenstand der vorliegenden Arbeit. Ebenso werden weder mikropolitische Vorgehen wie die vermeintliche Selbstenteignung der Wissensbestände innerhalb von Organisationen, noch das damit in Beziehung stehende strategische Gegeneinander weitergehend diskutiert. 
Smith-Doerr I994:380 und 2003: I5 f.). Die Bedeutung und die fundamentale Rolle von Status und Reputation, die Kooperationen in den Creative Industries übernehmen, wurde schon von Becker (2008) ausgearbeitet.

Langlebige Projekte kennzeichnet eine solide Vertrauensbasis, welche die Grundlage für geteilte Erfahrung und Identität wird. Vertrauen und Wissen bedingen sich in dieser Organisationskonfiguration gegenseitig, und so begünstigt gemeinsam generiertes Wissen die Entstehung von Vertrauen und das wiederum, dass Wissen preisgegeben wird. Auf einem gemeinsamen Erfahrungsschatz entstehen im Projekt Vokabulare und geteilte Anekdoten (Swap u. a. 200I; Patriotta/Lanzara 2006). Powell und Colyvas (2013: 292) definieren Vokabulare als grundlegende Verhandlungsordnung, die Dialoge strukturiert, gegenseitiges Verständnis erzielt und intersubjektives Bewusstsein fördert. Über enge und wiederholte Kontakte werden Vertrauensschwierigkeiten unterbunden und sowohl der Wissenstransfer als auch der Erfahrungsaustausch gefördert (Meyerson u. a. 2006). Ein Effekt davon ist die Konstruktion einer kollektiven (Projekt-)Identität, die wiederum das Vertrauen und die Handlungssicherheit stärkt. Identitäten stiften Sinn und entwickeln ein gemeinsames Verständnis für die symbolische Bedeutung der Zusammenarbeit (Dutton/Dukerich I99I; Lant 2005) $)^{53}$.

Geteiltes Verständnis und Vertrauen wird vor allem in heterogenen Teams über Geschichten hergestellt, denn die können sich aufgrund ihrer Verschiedenheit ursprünglich nicht anhand einer geteilten Sprache verständigen. Daher verweisen Vokabulare auf die Schwäche temporärer Organisationsformen wie Projektteams, denn sie zeigen die Diversität und Spezialisierung der Teammitglieder (Boland/Tankasi 1995). Anekdoten entstehen im Rahmen der Interaktion von kollektiven Akteuren in Projekten und Organisationen und werden von diesen weitergetragen. Sie haben die Fähigkeit, heterogene Einheiten zu einem Kollektiv zu verbinden und Sinn zu stiften, denn sie etablieren und erhalten Beziehungen zwischen bekannten und unbekannten Individuen (Rhodes/Brown 2005: I77). Ferner dienen sie dem Austausch von implizitem Wissen oder Problemlösungshilfestellungen, werden wiederholt, reproduziert und zu einem Handlungsstrang schematisch zusammengefügt (Hobday 2000: 885). Diese Praktiken setzen das Entstehen einer geteilten Projektsprache voraus, die sich in intraorganisationalen Narrativen und Storytelling (Sole/ Wilson I999; Brown/Gabriel/Gherardi 2009; Czarniawska 20I4b), »clusters of keywords« (Jones/Livne-Tarandach 2008), »institutional vocabularies«

53 | Westenholz beschreibt Identitäten als "socially constructed stories about individuals and their surroundings as they engage in social work practices. [...] Identities are socially constructed phenomena that simultaneously help people feel secure and act and understand their acting" (2006: 1018). Identitäten tragen insofern zum Gelingen von Projektaufgaben bei. 
(Meyer/Rowan I977), oder in Form von Ethnosemantiken und Alltagswissen (Maeder 20II) festschreibt. Die sprachliche Bedeutung spezifischer institutioneller Vokabulare erhält für die Darstellung von Ähnlichkeit als auch Verschiedenheit eine zentrale Rolle:

Vocabularies of structure which are isomorphic with institutional rules provide prudent, rational, and legitimate accounts. Organizations described in legitimated vocabularies are assumed to be oriented to collectively defined, and often collectively mandated, ends. [...] On the other hand, organizations that omit environmentally legitimated elements of structure or create unique structures lack acceptable legitimated accounts of their activities. (Meyer/Rowan 1977: $349 \mathrm{f.}$ )

Auf der Basis geteilter Vokabulare wird in Projekten ein gemeinsames Verständnis hergestellt, das den Erfolg des Projekts erhöht und kollektive Handlungsfähigkeit ermöglicht. Bei Wiederaufnahme des Projekts wird somit das Fortbestehen begünstigt. Dementsprechend sind Vokabulare und Anekdoten konstitutiv für das gegenseitige Verständnis und kollektive Sinninterpretationen in heterogenen Projektteams. Vokabulare ermöglichen den Austausch über normative Grenzen und koordinieren Kommunikation, Abläufe und Rollenverständnisse im Projekt (Czarniawska 20I5).

Die unterschiedlichen individuellen, organisationalen und professionellen Hintergründe bergen aber auch Kreativitätspotenziale. Aufgrund der Diffusion und Fluktuation der Projektmitglieder entstehen neue Formen der Informationszirkulation (Godart/Shipilov/Claes 20I4). Deshalb beinhalten interorganisationale Beziehungen großes Potenzial für vielfältiges Wissen: »Access to the knowledge of others is an important social capital resource, and individual-level relationships can be used to acquire knowledge that is useful to an employing firm« (Dokko/Rosenkopf 20I0: 679). Die heterogene Teamzusammensetzung fördert ein Out-of-the-Box-Denken, das zum Ideenreichtum, der Erfahrung und dem Wissen des erst projektspezifischen, später dann organisationalen Repertoires beiträgt. In Projekten treffen unterschiedliche Erklärungsansätze, Sichtpunkte und Antworten aufeinander, welche die unterschiedlichen Umwelten und Organisationen dialektisch wiederherstellen. Schließlich verhindern temporäre Projektmitglieder die Tendenz, dass sich Organisationseinheiten stark von der Umwelt isolieren und $\mathrm{zu} » I n s e l n$ « werden (Katz I982).

Die Bewerkstelligung von Kreativität heißt folglich auch, die räumliche Diffusion, andersartiges Wissens und normative Vokabulare und Ziele aufeinander abzustimmen (Swap u. a. 200I; Sydow u. a. 2004; DeFillippi u. a. 2007). Auch hier ergänzen sich Beständigkeit und Unbeständigkeit: Die langfristigen, institutionalisierten Strukturen verstetigen die effiziente Nutzung des vorhandenen Wissens, während die Austauschbarkeit der Arbeitskräfte Zugang zu neuen Ressourcen schafft und Wandel und Dynamik initiiert. 
Damit wird ein weiteres zentrales Merkmal der Modehybride angesprochen, das sich auf die Projektwiederholung bezieht: die Projektteams. Sie werden für den Projektzweck zusammengestellt und umfassen permanente und temporäre Organisations- respektive Projektmitglieder. Demzufolge besteht das Kollektiv aus einer heterogenen Mitgliederbasis. Die temporäre Mitarbeit bringt wirtschaftliche Vorteile mit sich: Organisationen reduzieren die Kosten, in dem sie auf langfristige Verpflichtungen verzichten und Experten nur temporär engagieren. Kollektive Projektteams können einmaliger Natur sein, z. B. im Film- oder Bausektor, oder sich in gleicher oder ähnlicher Formation wiederholen, wie z. B. in der Musik- oder Modebranche. Vertrauen und eine gemeinsame Sprache erleichtern die Wissenskumulation und den Wissenstransfer in und über die Projektgrenzen hinweg und ermöglichen dem Modeunternehmen und den Projektteilnehmern wertvolle Lernprozesse (DeFillippi 200I; Grabher 2004a 2004b).

Das Feldmaterial hat gezeigt, dass Moderfirmen einmalige, temporäre Kooperationen eingehen, jedoch wiederholte Zusammenarbeit und permanente Projektmitglieder befürworten. Einmalige Instanzen sind vor allem kürzere Kooperationen mit Künstlern z. B. für einen Stoffdruck oder eine Produktekooperation (beispielsweise Teppich, Honig, Parfüm). Aufgrund ihres künstlerischen Beitrags werden sie nicht wiederholt. Ein solches Beispiel der Projektarbeit ist die Modenschau, zu der $\mathrm{u}$. a. Models, Make-up- und Haarstylisten, Modefirmen und ihre Designer, Choreografen, Fotografen und DJs beitragen. Nicht selten werden Models rezykliert, um über das Gesicht einen Wiedererkennungswert des Unternehmens zu erschaffen. Fotografen, Choreografen oder DJs hingegen werden beliebig ausgewechselt, um mit der Tradition zu brechen. Das Anheuern verschiedener Kooperationspartner wird mit Diskontinuität und Erneuerung verbunden. Auf diese Weise wird beabsichtigt, so der PR-Manager im Interview, Neuerungen zu evozieren:

[A]lso noch ein anderes Beispiel. Wir shooten ja jeweils eine Kampagne für die Modekollektion und auch da ist der Anspruch, also die Anforderung an uns selber, dass wir lieber mit jemandem arbeiten, den/die wir nicht kennen, der/die jung ist, neu ist, als mit einem etablierten und angesagten Fotografen, der momentan gerade die coolen Sachen fotografiert. Und das ist unsere Haltung; sich immer neu zu hinterfragen und neue Sachen auszuprobieren, das ist etwas, was sehr stark bei uns ist, was ich als etwas Gutes empfinde.

Nicht selten werden Kooperationspartner nach der ersten Projektinteraktion dann doch wieder angeheuert. Die wiederholte Zusammenarbeit kann regelmäßiger oder unregelmäßiger Natur sein. Entsprechend verändern sich auch die Beziehungen zwischen den Akteuren und variieren zwischen stark und schwach. Die wiederholte Kooperation mit handwerklichen und technischen 
Partnern ist beliebt, wobei auf geteilte Erfahrung aufgebaut, Verständnis generiert und Rücksicht gegenüber divergierenden, professionellen Identitäten der Projektmitglieder genommen wird. Beispiele hierfür sind wiederholte Kooperationen mit Grafikern, Fotografen und Models oder mit anderen Modedesignern. Typische temporäre Projektmitglieder sind Schnitttechniker, spezialisierte Coutureschneider, Models, Fotografen oder Grafiker. ${ }^{44}$

Permanente Organisationsmitglieder haben unbefristete Arbeitsverträge. Dazu zählen Mitglieder des Designteams, d. h. des Designs (Creative Director), der Designassistenz (oft gelernte Schneider), der Produktion sowie das Management (oft auch Personal- oder Verkaufsverantwortliche) bzw. der Geschäftsleitung. Aber auch festangestellte Mitarbeitende des Modebetriebes wie Strickmaschinenoperateure oder die IT-Programmierer zählen dazu. In dieser Studie weist die kreative Leitung die höchste Permanenz auf, weil sie in die meisten Etappenziele des Kollektionsprozesses eingebunden ist. Dennoch ist die kreative Leitung nicht ausschließlich mit der Projektleitung gleichzusetzen. ${ }^{55}$

In den Musterungen fällt auf, dass die Prototypen nicht immer gemäß Vorschriften gefertigt wurden. Diese Notizen sind Hinweise auf fehlende Informationen, andersartige Interpretationen und zeugen von der Differenz des Wissensstands zwischen den Projektmitgliedern. Dennoch steht es dem Designer zu, diese Fehler zu übernehmen oder abzulehnen, weshalb sich die Designerin wiederholt fragt:

"Sollen wir die Reißverschluss-Band-Farbe ändern, oder ist es eben ein Look?"

Wie das oben angeführte Beispiel zeigt, können andersartige Interpretationen durchaus als positiv gedeutet werden. Somit sind Sitzungen und deren Protokolle ein wichtiges Instrument der Wissens- und Lernprozesse.

In temporären Formen der Zusammenarbeit werden Koordinationsprobleme erkennbar. Ein einschlägiges Beispiel ist die aufgezeichnete Situation einer seltenen Projektkooperation zwischen dem Fotografen und der Designerin:

54 | Räumliche Bedingungen spielen eine Rolle, wenn es um die Arbeitsteiligkeit in den Creative Industries geht (Becker 1974; Moeran 2008; Moeran/Christensen 2013). Einige Aufsätze betonen die Vorteile räumlicher Trennung, so zum Beispiel zunehmende Variation, wenn die Ideenfindung und der operationale Betrieb getrennt sind (Watson 2007; Krause-Jensen 2013; Lee et al. 2015), und andere weisen auf die Verworrenheit und Komplexitätszunahme aufgrund disparater Projekteinheiten im Produktionsprozess hin (Chen 2012; Tschang 2007).

55 | Der Designer ist nicht per se die präsenteste Person im Unternehmen, aber er hat die permanenteste Rolle inne. Aus größeren Modeunternehmen ist bekannt, dass Designer regelmäßig ihre Anstellungen wechseln und somit selten Amtsälteste werden. 
Der Fotograf fragt die Designerin: "Passen diese Outfits zusammen?" Sie antwortet: "Gar nicht!"

Die Akteure haben zwar früher schon einmal zusammengearbeitet, der Kontakt war aber zu lose, als dass man von gemeinsamer Erfahrung und geteiltem Wissen hätte profitieren können. Diese Interaktionssequenz illustriert, wie die professionellen Agenden divergieren und nicht auf dasselbe Wissen zurückgegriffen werden kann.

Die Zeithaftigkeit der Projekte beeinflusst weiter die Entstehung vertrauter Kooperationsbeziehungen. Die zeitlich begrenzte Zusammenarbeit zeigt oft nicht nur verschiedene erklärte Ziele und Motive, sondern auch das fehlende Vertrauen unter den Interaktionspartnern. So spielen Vertrauen und Vertrautsein ebenso eine Rolle in Interaktionen der Modeindustrie. Ein Beispiel des Unverständnisses und der Fehlinterpretation konnte bei einer Modenschauhauptprobe (Fitting) zwischen der Assistentin des Veranstalters und der Designerin beobachtet werden.

Oft ändert die Assistentin des Choreographen auch einfach den Look zu etwas, das die Designerin nicht für richtig empfindet. Dabei geht es um Details wie Pulli in die Hose stecken, ja oder nein?

Da der Kontakt zwischen Assistentin und Designerin schwach ist, teilen die beiden Projektteilnehmer nur wenig Wissen, was nicht dazu führt, dass sich das Problem der Ordnung des Looks nicht löst. Dies hat den Effekt, dass Teamidentitäten bestärkt und schwache Beziehungen künftig vermieden werden.

Repetierte Kooperationen helfen, Kommunikationsschwierigkeiten vorzubeugen und Kommunikation zu verbessern: Je älter die Zusammenarbeit zwischen Projektpartnern - je höher die Frequenz der Wiederholung der Projektarbeit -, desto größer der Erfahrungsschatz, das geteilte Wissen und das bestehende Vertrauen. Die Modefirmen können deshalb antizipieren, wie die Projektaufgabe aufgefasst, interpretiert und umgesetzt wird. Die Zuweisung von Produktionsaufträgen wird an dieser Handlungserwartung ausgerichtet. Während die einen das Schnittbild sehr gut lesen können und wenig zusätzliche Informationen benötigen, beziehen sich andere auf Bildmaterial von Details und Nachzeichnungen oder alte, verwandte Vorlagen. In einer Musterung wird angesprochen, dass trotz langjähriger Zusammenarbeit ungewiss bleibt, welche Informationen in welcher Art und Weise interpretiert und verarbeitet werden.

Gerade bei den Modell-Beschreibungen ist unklar, welche Info die Produzenten nun brauchen. Da unterscheiden sich die Lesarten der Länder: Die europäischen Produzenten sind sehr gut darin, das Offensichtliche zu verstehen, weshalb man bei innen 
eher Details beschreiben muss, oder das, was von ihrer klassischen Schneiderkunst abweicht (z. B. Handnähte). Beim Hersteller in Asien ist es andersrum: Was für das Designteam verständlich ist, wird missverstanden. Als Lösung wird so viel wie möglich ausformuliert und mitgeschickt, damit keine Missverständnisse entstehen.

Oft wird etwas nicht verstanden, das für das Schweizer Modeunternehmen total verständlich zu sein scheint. So wurden zum Beispiel Schraffierungen als Muster auf den Stoff gedruckt; Bettlaken wurden nicht als Bettdeckenanzug, sondern als einfache Laken genäht; oder ein Teddy wurde als Stofftier genäht, in dessen Bauch (ähnlich einem Känguru) sich aber eine Einkaufstasche aus Stoff hätte verstauen lassen sollen. Das Resultat war ein Teddy und eine separate Tasche im selben Stoff.

Nicht immer sind die visuellen und verbalen Ausführungen einer Idee für andere Projektmitglieder verständlich. Gründe für Wissensdifferenzen können kultureller, kommunikativer oder professioneller Natur sein. Kann nicht auf ein gemeinsames Technikverständnis oder Erfahrung zurückgegriffen werden, wird ein neuer Wissensvorrat angelegt. Dafür sind Sammlungen von designunterstützendem Material besonders geeignet. Anlagen wie beispielsweise ein Archiv - von der Designerin als cloud bezeichnet, auf welche die engen Kontakte schnell zugreifen können und die ein gegenseitiges Verständnis erleichtern - sind Orte der Inspiration und Erinnerung, auf die später verwiesen werden kann.

Die Zeithaftigkeit der Projektarbeit erschwert die Wissensangleichung. Im Modeprozess fungieren Prototypen als Kommunikationsmedien, die Informationen visualisieren, Wissen erläutern und zwischen abweichenden Wissensbeständen respektive -lücken der Akteure vermitteln und abgleichen. Bei einem Treffen zwischen der Schneiderin und der Designerin konnte beobachtet werden, wie auf diese Weise das weitere Vorgehen mithilfe von Modellen verhandelt wird:

Die Hose soll à la Herrenanzug sein, dazu gibt es einen Blazer, den es im Sommer 17 schon gibt, aber der etwas angepasst werden soll und deshalb auch den Namen ändert. [...] Die Schneiderin fragt, wie das Jackett dazu aussieht. Die Designerin will beim nächsten Treffen das Original von Sommer 2017 mitbringen, damit sie weiß, wie der Look aussehen soll.

Prototypen fungieren dabei als Objekte, die zwischen den individuellen und organisationalen Motiven im Projekt vermitteln und Wissen transportieren 
und illustrieren. Prototypen werden zu aktiven Beständen der Kommunikation und dem Wissenstransfer in heterogenen Projektkonstellationen..$^{6}$

Das Original ist eine permanente Form und impliziert bestimmtes Wissen, dessen sich die Schneiderin nun bedienen kann, während die Abänderung/ Weiterentwicklung des Schnitts zum Projekt wird. Durch dieses Projekt wird die permanente Struktur unterbrochen und Wissen verändert und hinzufügt.

In ähnlicher Weise koordinieren Analogien und Metaphern die Kommunikation und Wissensangleichung. Sie stellen dann Sinn her, wenn technische Komplexitäten sprachlich nicht erfasst oder nicht geteilt werden. Eine Beobachtung soll illustrieren, wie dabei mithilfe von Metaphern verhandelt wird:

Deshalb fragt die Designerin den Strickmaschinenprogrammierer, ob ihre Idee umsetzbar ist: das Waffelmuster satt zu binden. Der Strickmaschinenprogrammierer fragt mit leicht ironischem Unterton, ob es denn ein Pulli oder eine schusssichere Weste werden soll?

Auf den Begriff Waffelmuster wird mit den Analogien Pulli oder schusssichere Weste reagiert. Darunter können sich beide, Designer wie Techniker, etwas vorstellen. Sinnbildlich wird immer wieder auf renommierte Modehäuser (à la Coco Chanel, Chaneleffekt, wie die Atelierbilder von Dior) oder auch auf Stilarten (seemännisch, Maritimlook, französisch, Gauchoart, in Reitermanier oder auch wie Männerkleider für Frauen) verwiesen, um eine Analogie der intendierten Vorstellung des Prototyps herzustellen. Mittels Sprache wird hier Sinn herund Wissen bereitgestellt.

Weiter entstehen auch spezifische Vokabulare in Projekten. Es ist auffällig, dass sich beide Modefirmen ständig französischer Ausdrücke bedienen. ${ }^{57}$

Diese Ausdrücke erinnern an die französische Couture-Tradition, den historischen Ursprung des heutigen Modesystems. Die organisationale Sprache besteht als Teil des Jargons eines Kultursystems und macht eine institutionelle Verortung möglich. Demgegenüber steht die Sprache temporärer Organisationskonfigurationen. Während eines Fotoshootings sucht eines der Projektmitglieder überall nach dem Comic-esken, also der Möglichkeit, das Thema der Kollektion zu inszenieren. Die Projektaufgabe widerspiegelt sich in der Sprache und schreibt bestimmten Vokabularen (low-tech, Panzerknacker) eine projektbezogene Bedeutung zu. Folgende Beobachtung wurde bei einem Fotoshooting gemacht:

56 | Diese Diskussion wurde in der Organisationsforschung auch unter dem Begriff "boundary objects" geführt (Carlile 2002).

57 | So z. B. die Modellbeschreibungen la directrice, la céramique, la couturière, la vendeuse, la joaillereuse, Kollektionsthemen la route de la soie oder Kollektionsprogramme chaque mois, wie auch Farbbezeichnungen marine profond. 
Der Grafiker hat ein Monogramm ähnlichen Print entworfen. Der Print hat etwas comiceskes; dicke schwarze Linien umranden die Zeichnungen. Das Konzept des Fotoshootings ist es, diese Comic-Elemente in den fotografierten Bildern zu zeigen. Dazu wird der Comic-Charakter mithilfe physischer Objekte - in Kissenform genähte objets - oder in der Postproduktion integriert. [...] In der Küche entdeckt der Grafiker den alten Toaster und schlägt vor, eine Szene nachzustellen, bei dem der Toast ganz in "comicesker" Manier aus dem Toaster spickt. Er übt mit der Comic-Hand - eines der genähten objets - den Toaster-Hebel zu betätigen.

Hier ist das Comichafte im Fokus und entwickelt dabei sein eigenes projektbezogenes Vokabular. Comic-esk oder mad sind wiederholt benutzte Ausdrücke, während das Fotoshootingteam versucht, eine Bildsprache zu entwickeln, die diesen Comiccharakter aufnimmt. Der Grafiker übernimmt die Aufgabe des Suchens nach möglichen Comic-esken Sujets und findet auch im Keller eines:

Unten im Keller entdeckt der Grafiker eine Türe mit einem alten Safe-Schloss. Er mag es und will die Panzerknacker auch mit von Partie haben! Das sei ja total low-tech. Die Suche des Grafikers nach passenden Szenen hat etwas "Wahnsinniges" an sich; der Fotograf kommentiert ruhig, dass Hinweise aus der Umwelt [wie das Safe-Schloss] vielleicht besser wirken, wenn sie nicht auf jedem Bild zu finden sind.

Die Comic-Konnotation und das Vokabular entwickeln sich innerhalb dieses Projekts, dem Fotoshooting, und verlieren temporär an Relevanz, wenn sich das Team auflöst. Bei weiterer Zusammenarbeit kann es sein, dass auf die Erfahrung dieses Fotoshootings zurückgegriffen wird.

Ebenso sind die Produktnamen Teil des Projektvokabulars (vgl. Kap. 7.2.3). Immer wieder werden die Produktnamen genannt, die die Absichten mit den Prototypen verdeutlichen und die Kommunikation erleichtern. Die Designerin und die Schneiderin verweisen stets auf die Namen der Modelle, die die Veränderung der Schnitte in sich tragen:

Nun kommen die Designerin und die Couture-Schneiderin zum neuen Kleidchen-Modell. Die Designerin bezieht sich auf die Atelier-Bilder von Dior. Da sind die Schneiderinnen immer in adretten, rund- und hochgeschlossenen Kleidchen abgebildet, alle gleich, dunkelblau/marine, etwas prüde. Der Schnitt darf in Anlehnung an das Kleid Mireille sein, aber vorne soll es mehr gezögelt werden. Die Designerin will da viel Kreativität investieren und glaubt, dass ihr bestimmt eine Idee einfallen wird.

Der Name Mireille bezeichnet das Modell eines Kleides, das die Grundlage für die Weiterentwicklung der vorliegenden Skizze ist. Über den Namen wer- 
den die schnitttechnischen Änderungen und Besonderheiten abgespeichert. Geteilte Vokabulare sind der Ausgangspunkt für die Wissensgenerierung und -speicherung sowie den Transfer von Ideen und Informationen.

\subsubsection{Kreativität aus projektbasiertem Organisieren}

Die Kombination kontinuierlicher und temporärer Aktivitäten ist eine Besonderheit der Kulturproduktion, die auch auf die Entwicklung und Produktion in der Modebranche zutrifft. Die projektbasierte Koordinationsform ist die typische Organisationsform des Modesektors, weil die temporären und wiederholten Interaktionen immer in die Struktur der formalen Organisation eingebettet sind..$^{8}$

Projektbasierte Organisationsformen sind das Resultat eines Wechselspiels zwischen Projektaktivität und Formalstruktur. Ihre Repetition fördert Permanenz, während die Befristung der Projekte die Permanenz unterbricht. Die Wiederholungen und Einbettung ermöglichen die Entstehung starker Beziehungen. Diese weisen stabilere Vertrauensstrukturen als Einzelprojekte auf und haben einen nachhaltigeren Effekt auf den interorganisationalen, übergenerationalen Ressourcentransfer. Die Kommunikation und Koordination zwischen Projekten werden durch gesteigertes Vertrauen, geteiltes Wissen und eine gemeinsame Sprache erleichtert. Kooperationspartner transportieren Wissen über die Organisationsgrenzen hinaus und schaffen Zugang zu neuen, unbekannten Informationen. Der Wissensvorrat ist folglich nicht mehr nur in einer Organisation vorhanden, sondern als interorganisationales und intergenerationales Kollektivgut auf verschiedene Organisationen verteilt. Auf der Basis von Wiederholungen entstehen neben den Projekten Netzwerke, die für die kreativen Neuerungen von Wichtigkeit sind. Einerseits bestehen latente Personalnetzwerke, aus denen temporär Projektmitglieder aktiviert werden, andererseits entstehen Produktionsnetzwerke, die Folge starker Arbeitsteilung sind. Solche Ressourcennetzwerke verbinden verschiedene Akteure, Wissensbestände und Apparaturen. Schließlich werden diese zu einer (legitimierenden) Instanz, in welcher sich die neuen, kreativen Güter verbreiten.

Modefirmen installieren dadurch einen Mechanismus, stilistische Uniformität und rigide Reproduktion zu vermeiden. Der Wandel in den Projektteams begünstigt die Fluktuation von temporärem Personal und erweitert

58 | An dieser Stelle ist anzumerken, dass in Projekten die Aktivitätsstruktur veränderbar ist, während die Formalstruktur permanent bleibt. Umgekehrt verhält es sich mit Technologien, denn da wird durch die Formalstruktur eine zeremonielle, temporäre Außendarstellung gepflegt, die lose gekoppelt von der internen Aktivitätsstruktur, der permanenten Technologie, besteht. 
den Ressourcenfluss. Anders formuliert: weil die Organisationsstrukturen im Kern nicht sehr flexibel und neuheitsfördernd sind (Routinen und standardisierte Handlungsabläufe, Puffern zentraler Technologien, Kontinuität im Betriebskern), muss eine bezüglich neuer Ideen vielversprechende und tolerante Schaltstelle zwischen Organisation und organisationalem Feld bzw. institutioneller Umwelt eingebaut werden, sodass die Neuheitsförderung sichergestellt wird. In diesem Sinne werden Projektmitglieder und Kooperationspartner als Zugang zu verschiedenen sozialen Räumen eingesetzt. Gatekeeper werden über Kooperationen zur Kreativitätshervorbringung zugeschaltet und verbinden die lokale Organisationsebene des Projekts mit der organisationalen Umwelt. Besonders Künstlerkooperationen sind legitimitätsfördernd, denn sie versehen die Organisation mit »external blessings «, deren Effizienz in Bezug auf die Produktionsverfahren unklar ist, die aber dennoch einen wesentlichen Einfluss auf die interne und externe Legitimität ausübt (Meyer/Rowan 1977: 355; Bromley u. a. 2012: 482).59

Schließlich ermöglichen latente Produktionsnetzwerke und die Fluktuation der Projektmitarbeiter, dass neue Ressourcen in die Organisation gelangen, die wiederum die Voraussetzung für Veränderungen schaffen. Ob nun regelmäßig die gleichen Akteure oder absichtlich unterschiedliche eingestellt werden, wird als Argument zurechtgelegt, das entweder die Zuverlässigkeit oder die Erneuerung betont. Was bleibt, ist eine Gratwanderung zwischen Permanenz und Zeithaftigkeit, die es organisational auszubalancieren gilt. Da die Zeit die Grundlage der projektbasierten Organisation ist, wird Kreativität zum Termingeschäft.

\subsection{Technologische Aspekte Und die Neuheitsroutine}

Verfolgt man die Art und Weise, wie Kreativität in Modefirmen systematisch hervorgebracht wird, fallen drei grundsätzliche Aspekte auf: Erstens beruht die Mehrzahl der Arbeitstätigkeiten auf verlässlichen, jedoch variierbaren Routinen, die sich im Zeitverlauf wiederholen und ähnlich einer Grammatik das Handeln anleiten. Zweitens sind Technologien im Hervorbringungsprozess, v. a. in der Entwurfs-, Entwicklungs- und Produktionsphase essen-

59 | Von solchem "Segen « wollen auch New Verticals wie H\&M oder Zara zehren, wenn sie temporäre Kooperationen mit Stardesignern eingehen. Bei solchen Kooperationen handelt es sich um einmalige, interorganisationale Projektformen, die nur für kurze Zeit bestehen und sich nach dem festgelegten Zeitraum auflösen. Das Hervorbringen von Kollektion in mittelgroßen Modeunternehmen folgt festgelegten Routinen. Jedoch werden von Zeit zu Zeit Künstlerkooperationen durchgeführt, um erhöhte Aufmerksamkeit zu erzielen. 
ziell. An Technologien wird die künstlerische Umsetzbarkeit des Designs ausgerichtet. Drittens finden sowohl die routinegeleiteten Aktivitäten als auch die technologischen Kalibrierungen in Teams statt. Projekte stellen eine variable Struktur für Prozesse in den Creative Industries und demnach die Modeindustrie bereit, die die Schließung bzw. Öffnung an der Grenzstelle zwischen Organisation und Umwelt temporär gestatten. Projekte terminieren den Einfluss externer Umweltdynamiken auf interne Organisationsaktivitäten. Insofern sind Organisationen keine in sich abgeschotteten Einheiten. Die hier beschriebenen Prozesse veranschaulichen das Paradox der Gleichzeitigkeit von Kontinuität und Wandel, wobei die Berücksichtigung beständiger Elemente die Akzeptanz sich wandelnder Elemente erhöht (Child 1997). Eingespielte Methoden (routinierte Handlungsabfolgen, technische Apparaturen) und standardisierte Wiederholungsmuster (repetitive Rekrutierung temporären Personals) rücken in den Vordergrund, anhand derer kontinuierlich Neues generiert wird. Der Herstellungslogik, die diesen Aktivitäten zugrunde liegt, soll als Neuheitsroutine bezeichnet werden. Mit dem Kontingenzansatz wurden Routinen, Technologien und die Projektstruktur als zentrale, stabilisierende Organisationsformen der Modekreation herausgearbeitet, die zusammen mit den Verfahren der Neuheitsroutine die technologischen Aspekte der Kreativitätshervorbringung definieren. Auf der Basis des empirischen Feldmaterials wurde eine Übersicht geschaffen, die über die alltäglichen Arbeiten des Kollektionsprozesses informiert (Abb. 3 und 4$) \cdot{ }^{60}$

Routinen sind weniger rigide, als bislang vermutet wurde, und aufgrund ihrer performativen Umsetzung lassen sie auch Variationen und Neuinterpretationen von Handlungen zu. Das bedeutet, dass sowohl Planung als auch Emergenz die Kreativitätshervorbringung beeinflussen. Während die musterhaften Prozessabläufe geplant werden, werden Projektaufgaben flexibel gehandhabt. Routinen sind sowohl verlässlich als auch variationsfähig. Ähnlich verhält sich die Gestaltung einzelner Kollektionsteile, deren Materialqualitäten langfristig bestehen bleiben, Muster und Farben jedoch von Zeit zu Zeit im Designprozess neu zusammengestellt und kombiniert werden. Mode scheint zwar immer im Voraus geplant zu werden, das verraten uns z. B. Abgabefristen und sequenzielle Prozessabfolgen, nichtsdestotrotz spielt der Zufall immer wieder in die Kreationsprozesse rein.

60 | Sofern nicht anders angegeben, handelt es sich bei allen Abbildungen um eigene Darstellungen. 
Abb. 3: Organisation der Modekreation (1): Von der Vorstellung der Entwürfe bis zum Muster

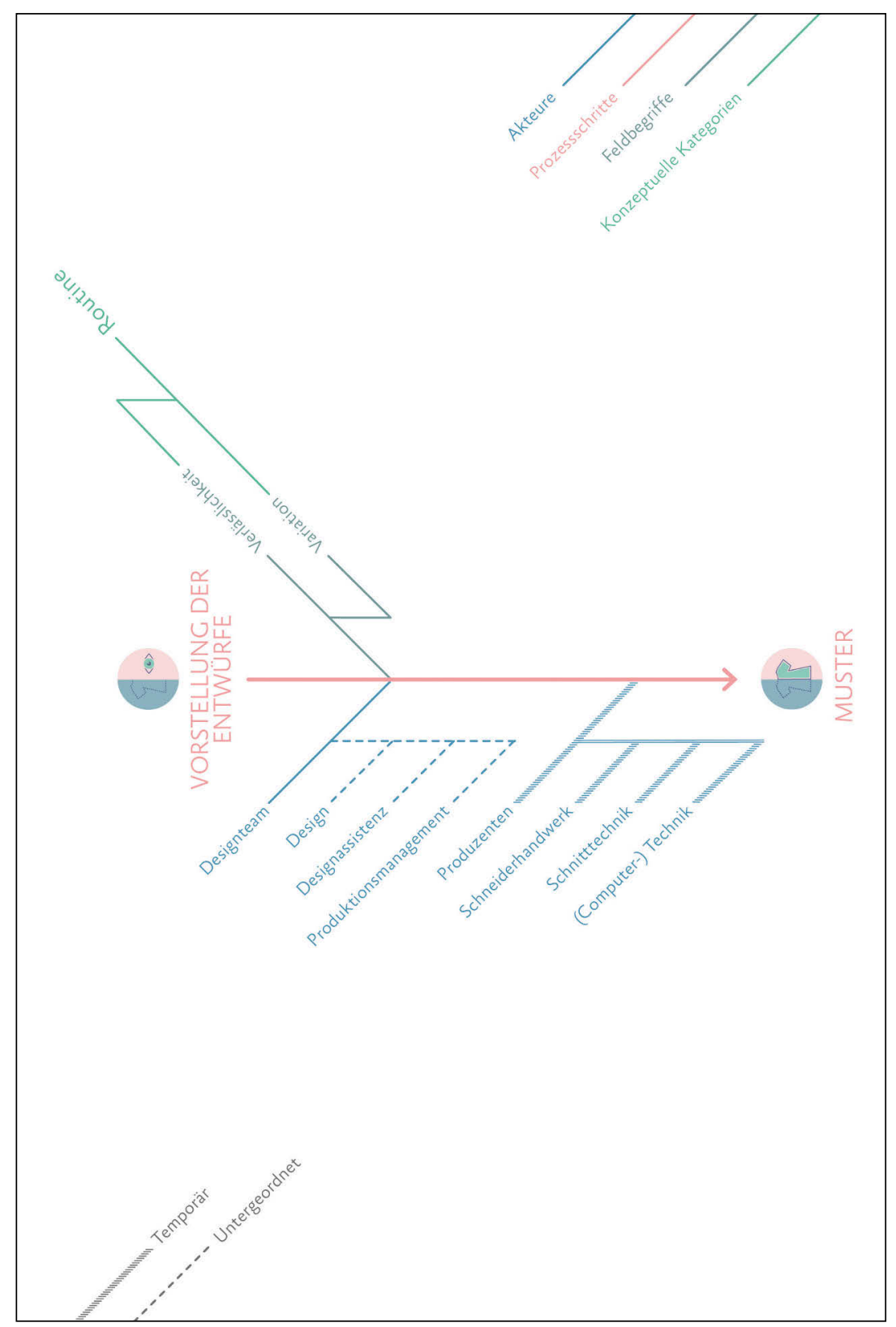


Abb. 4: Organisation der Modekreation (2): Vom Muster zum Kollektionsmuster

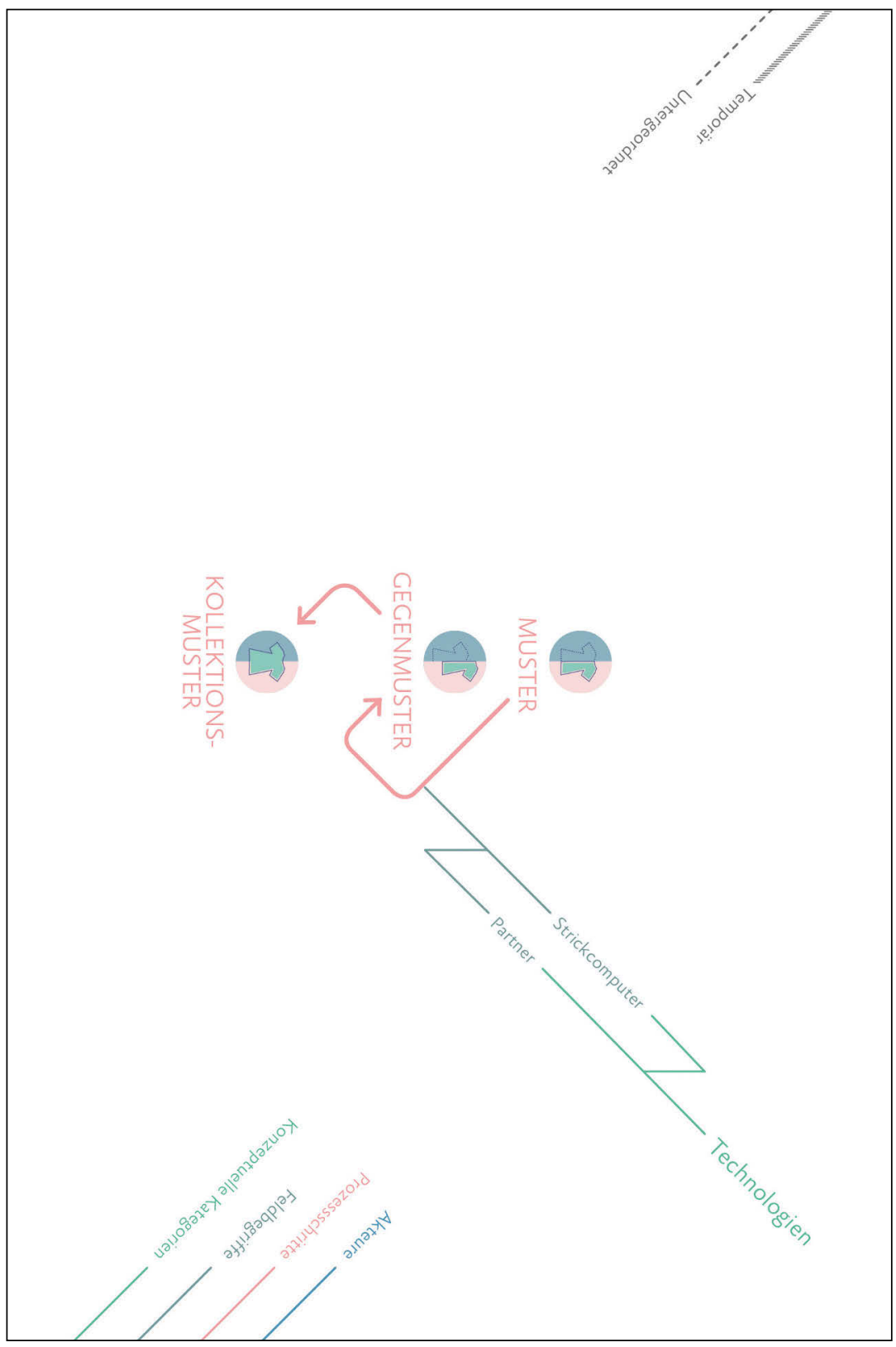


Weiter hat sich gezeigt, dass Technologien nicht nur bewahrt, sondern auch institutionell und organisatorisch abgeschottet werden. Diese Abschottung dient dazu, dass der Betrieb im Organisationskern trotz diskontinuierlicher, unklarer Nachfrage kontinuierlich aufrechterhalten wird. Das Feldmaterial illustriert die Bewahrung des institutionalisierten Wissens der Produzenten (Handwerkskunst, technische Expertise der Partner) und die zentrale Rolle von Techniken und Apparaturen (Strickmaschine), damit Inspirationen aus der Umwelt in die Organisation gelangen. In Musterungen und Gegenmusterungen werden Umwelteinflüsse in rekursiven Prozessen gegengeprüft und in die Technologietradition übersetzt. Übersetzungsprozesse haben immer Veränderungen zur Folge, weshalb kreative Güter ein Kompromiss zwischen Veränderung und Anpassung sind. Das Resultat dieser Prozesse ist das Kollektionsmuster, das zu kommunikativen Darstellungen benötigt wird (Kap. 7).

Darüber wurde versucht zu illustrieren, wie sich die Projektorganisation als Basisstruktur der Kreativitätshervorbringung in der Mode herausgebildet hat. Akteure der permanenten Organisation (die Formalorganisation des Unternehmens) kooperieren gerne mit verschiedenen temporären Akteuren (so z. B. freies Personal wie Schnittmacher, Fotografen, Models). Die Projektstruktur lässt nicht nur variable Teamkonstellationen zu, sondern reagiert auch auf die rekursive Entwicklungsstruktur und die austauschbaren Prozesssequenzen. In Arbeitsteams werden einzelne Aufgaben zum Etappenziel. Trotz wechselhafter Rekrutierungsmuster zahlt sich oftmals aus, bekannte Projektteilnehmer wiederholt anzuheuern, denn dadurch können Risiken und Fehlschläge verringert werden. $\mathrm{Ab}$ und $\mathrm{zu}$ werden neue Kooperationspartner eingeladen, die es auch erst zu überprüfen gilt und dann entweder der künstlerisch-kreativen Legitimität dienen (und einmalig bleiben) oder das latente Netzwerk der Projektpartner langfristig erweitern. In jedem Projekt unterscheiden sich professionelle Agenden, erklärte Ziele und divergierende Erwartungen wie auch das Wissen und die Erfahrung der Projektmitglieder. Werden gleiche Teams über mehrere Wiederholungen hinweg mit derselben Aufgabe betraut, kann beobachtet werden, wie sukzessive ein geteiltes Vokabular unter den Projektmitgliedern entsteht, das Vertrauen zunimmt und der Informationsaustausch (auch langfristig und interorganisational) gefördert wird. Das Projekt ist eine Möglichkeit der temporären Öffnung der Organisation gegenüber externen Einflüssen. Deshalb sind Akteure des Unternehmens - entweder das ganze Designteam oder nur der Designer - stets an Projekten beteiligt, um die Einflüsse auf ihre technologische Umsetzbarkeit zu prüfen.

Die Neuheitsroutine kombiniert standardisierte Handlungsprogramme mit erneuernden Ideen und übersetzt diese in die technologischen Möglichkeiten des Betriebs. Sie bewährt sich, weil sie interne, technologische Sicherheit und Stabilität und gleichzeitig externe, kreative Anerkennung durch Veränderung herstellt. Die Dynamik der projektbasierten Organisationsstruktur ermöglicht 
das Zusammentreffen von verankerten Technologien und auswärtigen Einflüssen. Auf der Basis der Neuheitsroutine werden diese modifiziert und mit gewünschten organisatorischen Zielen in Einklang gebracht. Dadurch kann sich die Organisation zur Umwelt hin öffnen, während gleichzeitig Bewährtes fortgeführt wird. Durch die Neuheitsroutine eignet sich die Organisation fremde Ideen an und macht sie sich zu eigen. Diese Übersetzung und Aneignung - in der Mode ist sie Bestandteil der kreativen Designtätigkeit - führt zu neuen Kollektionen, die als modisch bezeichnet werden.

In diesem Kapitel wurde die Organisation der Modekreation, deren Entwicklungsstruktur sowie deren Produktionslogik erläutert und unter dem Begriff der Neuheitsroutine zusammengefasst. Anhand dieser Mechanismen wurde versucht $\mathrm{zu}$ erklären, wie Kreativität technologisch produziert wird. Die Neuheitsroutine bezeichnet die flexible Anpassungsstrategie, die gleichzeitig stabile Strukturen zulässt. Sie umfasst deshalb zwei Rationalitäten sowie Verlässlichkeit und Variation, Erneuerung und Tradition und Permanenz und Temporalität. Die daraus resultierende Vielfalt an Kombinationsmöglichkeiten wurde exemplarisch herausgearbeitet und zeigt, wie Modefirmen den Spagat zwischen Tradition und Innovation, Technologie und Kreativität schaffen und welche Rollen und Funktionen den beteiligten Akteuren dabei zukommen. Das Zusammenspiel von Routinen respektive Verlässlichkeit und Variation, Technologien respektive Strickcomputer und Partner und Projekten respektive temporärem und permanentem Organisieren führt schließlich von der Entwicklung zur Produktion kreativer Güter. Kreativität ergibt sich aus den musterhaften Handlungsanleitungen und deren bewusster Neuinterpretation; aus bewährten Entwicklungsstrukturen, Produktionsmethoden und neuen Bedeutungen; aus der permanenten Organisationsstruktur und einer Vielzahl temporärer Vorhaben.

Diese Ausführungen zur Organisation der Modekreation erklären jedoch nicht, weshalb die Modebranche als kreativ wahrgenommen wird, während bei anderen auch von Routinen, Technologien und Projektstrukturen geprägten Industrien dieses Attribut nicht legitim scheint. Deshalb wird im nächsten Kapitel auf die kommunikativen Bedingungen und Fragen der Darstellung von Kreativität in der Mode eingegangen. Die Creative Industries und insbesondere der Modesektor unterhalten Zeremonien und bauen Fassaden der Kreativität auf. Sie sind als Reaktion auf Rationalitätsmythen der institutionellen und kulturellen Umwelt zu verstehen, deren Missachtung zu Legitimitäts- oder Ressourcenverlust führen kann. 



\section{Darstellungsformen der Mode}

Die technologische Herstellung von Kreationen über Routinen, Technologien und Projekte, welche die Basis der operativen Verfahren ist, ist unvollständig. Sie erklärt nur lückenhaft, weshalb die Creative Industries als kreativer gelten als andere Industrien. Deshalb konstruieren Firmen einerseits Sprachspiele, d. h. Narrative und Storytelling, um eine kreative Fassade aufrechtzuerhalten und den Glauben an Kreativität sprachlich zu verankern. Andererseits inszenieren sie zeremonielle Darstellungsformen und Shows, um Authentizität und Originalität zu kommunizieren. Diese kommunikativen Ausgestaltungen stehen in diesem Kapitel im Fokus.

Das Prinzip zuverlässiger Entwicklung und Produktion von Kreationen demystifiziert den gängigen gesellschaftlichen Kreativitätsmythos und weicht stark von den Erwartungen - was und wie Kreativität ist bzw. entsteht - des relevanten Publikums. Um den Erwartungen des Publikums gerecht zu werden und die Authentizität und Legitimität im Feld zu erhalten, müssen Modefirmen ein kreatives Selbstbild inszenieren (Khaire 20I4; Jones u. a. 20I6). Inwiefern unterscheidet sich die Mode also von anderen Creative Industries und weiter noch von anderen, >profaneren< Sektoren? Und wie schaffen es Modefirmen, Kreativität zu reklamieren, wenn sie ihre Prozesse per Routine, Technologiestandardisierung und repetitiven Projektorganisationen organisieren? In diesem Kapitel werden institutionelle Faktoren der Kreativitätshervorbringung untersucht. Die Selbstkontextierung und die Bezugnahme auf die eigene Vergangenheit wie auch das Organisationsfeld wirken hierbei als Referenzrahmen, der die Kommunikation neuer Kreationen anleitet. In diesem Kapitel werden narrative Fragmente der Orientierung, inszenierende Shows und finalisierendes Storytelling als kommunikative Darstellungsformen in der Mode rekonstruiert.

Das Feldmaterial liefert Hinweise, wie Organisationen Sprache nutzen, um Kreativität zu generieren. Mit Storytelling und Inszenierungsmethoden koppeln Organisationen die technologischen und kommunikativen Kreativitätsaspekte (lose) miteinander und reagieren dadurch zeremoniell auf Erwartungen des Publikums. Der Kreativitätsmythos wird mit Zeremonien und Fassaden reproduziert. Der analytische Fokus konzentriert sich auf die täglichen Prak- 
tiken der Verwendung von Sprache, genauer auf die Wahl institutionalisierter Argumentationsbausteine und dem Spiel damit (organisierende Geschichten). ${ }^{61}$

Geschichten sind somit nicht Resultat strategischer Rhetorik, sondern vielmehr sind sie als prozessuale und unfertige, narrative Fragmente zu beobachten, die die Zukunft heraufbeschwören oder aktualisieren: »Whether stories are interpreted as constituting, mapping, encouraging, managing, upsetting, preventing or inviting change, they are an ever present feature of patterns of becoming, always evocative of actual futures and possible worlds « (Brown/ Gabriel/Gherardi u. a. 2009: 325; Hervorh. der Verf.). Der Sprache kommt eine teils geplante, z. B. als strategische Rhetorik und Außendarstellung, teils emergente Rolle zu, z. B. in narrativen Fragmenten. ${ }^{62}$ Die sukzessive, aus narrativen Fragmenten entstehende Geschichte wird symbolisch und materiell an kollektionsspezifischen Hinweisen fixiert, sodass retrospektiv der Eindruck einer von Beginn an geplanten Geschichte entsteht. Die Darstellungsformen der Mode sind »kreativisierende« Zeremonien, die die lose Kopplung der >unspektakulären< Entwicklungs- und Produktionsweise und der >spektakulären< Außendarstellung überbrücken und eine kreative Fassade der Gutgläubigkeit pflegen. Die Rationalität standardisierten Organisierens und die Authentizität der kreativen Güter sind lose gekoppelte, scheinbar konträre Aspekte, die konstitutiv für die Kreativitätshervorbringung werden (Suddaby u. a. 20I7). Folgt man diesem Argument, so sind materielle (Accessoires, Etiketten, vestimentäre Details des Designs) und symbolische Markierungen (Thema, Modellnamen, Farbbezeichnungen) narrative Fragmente, die sich in der zeremoniellen Ausgestaltung des finalisierenden Storytellings zu antizipierten Geschichten und retrospektiven Idealisierungen zusammenschließen. Narrative Fragmente und Geschichten sind integraler Bestandteil der Organisation, der dazu eingesetzt wird, Signale zu senden, auf deren Grundlage Handlungen bewertet und Wichtigkeiten zugeschrieben werden können (Mützel 20I7). Abschließend erläutere ich an diesen Darstellungen die kommunikativen Aspekte von Kreativität.

Deshalb gilt das besondere Interesse dieser Arbeit den Praktiken und Prozessen der Narration und nicht deren Inhalt, also dem »storytelling rather than

61 | Jede neue Wiedergabe der Erzählung verfestigt sie als "Sediment von Normen und Praktiken“, was langlebige Erzählungen besonders interessant für die Organisationsforschung macht (Czarniawska 2015: 87).

62 Als Folge der narrativen Fragmente und der Zeithaftigkeit der Mode entstehen selten "grand narratives" (Boje 1995). Ein modehistorisches Beispiel eines "grand narrative" könnte der New Look der Nachkriegszeit sein, der sowohl gesellschaftspolitisch als auch sozialökonomisch modebezogen auf die aktuelle Situation reagiert hat. Ein jüngeres Beispiel wäre die Punkmode. 
stories « (Polletta u. a. 20II: III, Hervorh. der Verf.). Neuerungsnarrative werden in dieser Arbeit als a) die aus den Fragmenten zusammengestellte Geschichte sowie b) der Prozess des Erzählens dieser kollektiven, mehrstimmigen Sinnesverarbeitung und der alltäglichen Bedeutungsherstellung in Organisationen definiert. ${ }^{63}$

Zusätzlich hebt Storytelling stilistische Besonderheiten hervor, welche die Andersartigkeit der Modefirma in Konkurrenzräumen beleuchten. Im Rahmen jeder neuen Kollektion wird die »stylistic innovation « (Cappetta/Cillo/ Ponti 2006) präsentiert, die sich durch diese Eigenheit von anderen eigenen, ehemaligen wie auch fremden Kollektionen unterscheidet. Die Inszenierung der Neuerungen ist der Peak der entwickelten Kleidung - denn das Resultat der rekursiven Entwicklungs- und Produktionsprozesse ist eine Sammlung erfolgsversprechender Modelle. Kreativitätsshows, d. h. Modenschauen und Fotoshootings, aber auch die Laden- und Schaufenstergestaltung, sind Teil der kreativen Selbstdarstellung. In diesen Shows werden die Neuerungen als Geschichte und als kreative Darbietungen inszeniert (Kühl 20I5). Narrative und zeremonielle Darstellungsformen dienen der glaubwürdigen Außenkommunikation, denn sie sind sowohl institutionalisierte Formen der Veränderungsdarstellung und als solche Verschiedenheitsmarker als auch Merkmale des Kollektionszusammenhangs und als solche Ähnlichkeitsmarker. Die vermittelte Anders- und Einzigartigkeit wird zur Grundlage für die Verortung im Markt und die kognitive Bestimmung der eigenen Marktnische.

\subsection{Sprache Und Kreativität}

Der Sprache kommt eine bedeutende Rolle in der phänomenologischen Organisationsforschung zu. So schreiben Berger und Luckmann: »Die objektivierte soziale Welt wird von der Sprache auf logische Fundamente gestellt. Das Gebäude unserer Legitimationen ruht auf der Sprache, und Sprache ist ihr Hauptinstrument« (20I3: 69 [I966]). Sprache ist die Basis unserer sozialen Wirklichkeit. ${ }^{64}$ Demzufolge markieren Geschichten, Narrative oder Erzählungen keine außergewöhnlichen Ereignisse oder werden nur bei ambivalenten Umständen beigezogen; vielmehr verbalisieren sie die soziale Wirklichkeit des organisationalen Alltags als gelebte Erfahrung (Rhodes/Brown 2005: I82). Narrative

63 | Storytelling unterscheidet sich von den nach innen gerichteten Erzählungen wie Anekdoten, die vor allem in professionellen, temporären und heterogenen Gruppen entstehen, eine gemeinsames Bewusstsein herstellen und zur Sinnstiftung beitragen. 64 | In der Soziologie wird deshalb nicht von ungefähr vom "linguistic turn" gesprochen, und in der Organisationsforschung hat diese Wende zu einer Stärkung der Bedeutung von Sprache und Sinn in Organisationen geführt (Alvesson/Kärreman 2000). 
in Organisationen sind beispielsweise Jahresberichte, Jobinterviews, Pausenanekdoten oder Werbeslogans, die sich aber nicht immer zu Geschichten (mit einem Anfang, einem Hauptteil und einer Pointe) zusammenfügen. ${ }^{6}$

Czarniawska (20I5: 8I) bilanziert, dass die narrative Wende in den Sozialwissenschaften die Aufmerksamkeit auf etwas gerichtet hat, das bis dahin unbemerkt blieb, weil man es für selbstverständlich hielt. Die Folge war die Zunahme des Interesses der Organisationsforschung an Narrativen, Geschichten und Storytelling.

Als sprachlich-phänomenologische Konstrukte erschaffen und gestalten Geschichten soziale Wirklichkeit (Zilber 2009). So werden Geschichten »von rhetorischen Strategien zu alltäglichen Begründungen« (Hasse/Schmidt 2008: I9), die die alltägliche, jedoch besondere Arbeitsweise der Organisationen offenlegen und Kapitalgeber, Kritiker und Konsumenten über neue Unternehmen und Produkte informieren (Lounsbury/Glynn 200I). ${ }^{66}$ Geschichten dienen als Instrumente, mit welchen kontextuell und situationell Bedeutungen hergestellt werden (Mützel 20I7), die dann zur Grundlage für organisationale Interpretationen und Entscheidungen werden (Patriotta 2003). Weiter können Geschichten zu einem Diskurs werden (Mützel 20I5), die Ausbildung organisationaler und individueller Deutungen des Selbst begünstigen (Czar-

65 | Die verschiedenen Lesarten von Geschichten in Organisationen stellen bis heute das Hauptproblem narrativer Ansätze dar. In der Literatur sind vornehmlich drei Hauptunterscheidungen auszumachen, die sich auf Geschichten als Daten respektive mehr oder weniger organisierte Texte (stories) oder Performances (Storytelling) (Boje 1991; Polletta et al. 2011; Czarniawska 2015), auf Geschichten als theoretisches Konstrukt der Organisationsanalyse (z. B. Czarniawska 1998, 2015; Pentland 1999) oder als methodologisches Vorgehen (Boje 2001; Czarniawska 2007b ) beziehen. Da Geschichten aber immer einen Zustand - einen bestimmten Inhalt - zu einer bestimmten Zeit abbilden, sind sie per se immer unvollständig (Mützel 2017: 63). Natürlich gibt es auch Mischformen (Rhodes/Brown 2005). Allgemein lässt sich eine Tendenz des "narrativen Ansatzes in der Organisationsforschung " feststellen, der sich in den letzten Jahrzehnten verdichtet hat, aber dessen Mehrwert für dieselbe Forschung aufgrund uferloser Diversifizierung schwierig festzulegen ist (Czarniawska 2015: 81; Brown/ Gabriel/Gherardi 2009).

66 | Geschichten als empirisches Feldmaterial zu verwenden, bietet eine einzigartige Nähe zum Forschungsgegenstand. Narrative Methoden verknüpfen das wissenschaftliche Interesse und das subjektive Alltagswissen, worin eine der zentralen Stärken der Methode und des Gegenstands liegt: "Narrative research is, by and large, an empirical tradition that examines how experience is reflexively constructed into stories that may or may not be commensurate. It provides a methodological position through which to engage not with a presumed neutral realı world, but with the complex nuances of the lived، world" (Rhodes/Brown 2005: 180). 
niawska-Joerges 20II) oder bestimmte organisationale Genres wie Geschäftsberichte in eine allägliche Sprache übersetzen (Shaw/Brown/Bromiley I998). Zusammengefasst werden Handlungen über Geschichten koordiniert, orientiert und koordiniert (Mützel 20I7: 50 f.). Eine Vielzahl empirischer Untersuchungen weist deshalb auf den Zusammenhang zwischen Geschichten und Wissen, Sinngebung, Macht, Kommunikation und Wettbewerb hin (Martin u. a. I983; Sole/Wilson I999; Brown u. a. 2009). Demzufolge ist Storytelling als ein Schlüsselmechanismus der Gestaltung und Sinnstiftung sozialer Wirklichkeit zu verstehen (Weick I995). In ihr wirken Geschichten als »interpretativer Leim« (Mützel 20I5: 273). Wichtig für die hier vorliegende Arbeit ist ihre Fähigkeit, die Verbreitung innovativer Ideen zu koordinieren und den Zugang zu Alternativen zu erschließen (Bartel/Garud 2009) sowie Institutionen zu verfestigen (Zilber 2009: 205).

In der Praxis der Mode ist Storytelling ein organisierendes Werkzeug zu kreativ-künstlerischen Begründungszwecken (Czarniawska 20I5). Deshalb werden Organisationen auch als »storytelling systems« bezeichnet (Boje I99I). Sprache wird folglich auch als Grundlage für die Herstellung von Kreativität begriffen und erzielt bedeutende Wirkungen in den Creative Industries $u$. a. in der Wissensvermittlung oder der nach außen gerichteten Kommunikation. Organisierende Narrative dienen der internen Kommunikation, organisationale Narrative hingegen richten sich an die allgemeine Umwelt, wofür die Neuerung auf attraktive Weise aufbereitet wird.

Für die Organisationstheorie ist insbesondere die legitimitätsstiftende, erwartungsstillende Funktion von Sprache bzw. von Geschichten zentral. Um von Anspruchsgruppen akzeptiert zu werden und als rechtmäßige Feldmitglieder Anerkennung zu finden, orientieren sich Organisationen an Erwartungen und Bedingungen der institutionellen Umwelten, auf die mit Sprachspielen zur Neuerung reagiert wird. Unternehmen machen sich durch Sprache institutionell bedeutsam, gewinnen an Geltung, Verständlichkeit und Legitimität, die neue Industrien, Unternehmen sowie neue Produkte benötigen (Aldrich/Fiol I994; Petkova 20I6). Geschichten institutionalisieren und tragen zur Verständlichkeit von Organisationen und ihren Zielen bei:

Once articulated, understood, and repeated, entrepreneurial stories become institutionalized accounts that provide both explanations of, and rationales for, entrepreneurial activity; in turn, such comprehensibility (or understandability) is the basis for legitimacy. (Lounsbury/Glynn 2001: 551)

Solche Accounts sind »vorgefertigte Argumentationsbausteine«, die in verschiedenen Situationen zum Einsatz kommen und zum Zweck des Geschichtenerzählens neu kombiniert werden (Hasse/Schmidt 2008: 20). Powell und Colyvas schließen mit ihrer mikrosoziologischen Argumentation an Meyer 
und Rowans (1977: 349) Darstellungen des organisationalen Gebrauchs institutioneller Vokabulare an, die - wenn kongruent mit institutionellen Regeln - die kollektive Ausrichtung im Feld bedeuten und rationale und legitime Begründungen bereitstellen.

As these raccounts of performance or activity take hold, they become reified, that is received and accepted as normal by their participants and adopted and emulated by others who were not a part of their initial creation. In this sense, local measures become natural. Once natural, they become public, as the measures refine and reinterpret history, and evolve into models that others aspire to, and are recognized as guideposts of accomplishment. (Colyvas 2013: 292)

Die für organisationale Sprachspiele verwendeten und interpretierten Argumentationsbausteine stammen von cultural accounts und meta-narratives, in anderen Worten »stories that attempt to introduce a lasting order in one sweep« (Czarniawska/Sevón I996a: 3; Creed/Scully/Austin 2002; Suddaby/ Greenwood 2005). Organisationen verwenden die kulturell und institutionell vorgefertigten Argumentationsbausteine in ihren Geschichten, denn diese sind als »sprachliche Begründungen auch in Phasen der Aufrechterhaltung von Institutionen bedeutsam, weil sie zum Beispiel in Fällen der Abweichung [...] aktiviert werden können « (Hasse/Schmidt 2008: 20 f.). Die Allgemeingültigkeit und Flexibilität der Meta-Narrative bedeutet, dass sie übernommen und angepasst werden können und deshalb sowohl in unterschiedlichen Situationen (Modeunternehmen, Sportvereine) als auch auf verschiedenen Ebenen (individuell, organisational, national) Sinn stiften (Zilber 2009: 224).

Petkovas (20I6) Beispiel des E-Commerce im auf physischer und haptischer Erfahrung beruhenden Feld der Mode illustriert, wie Technologiefirmen durch die Implementierung legitimierender Narrative digitale Konsumformen etablieren. Die Mode reklamiert ihre Neuerungen mittels spielerischen Narrativen.

Zuletzt wird die aus Accounts und narrativen Elementen konstruierte Geschichte mit einer Pointe vervollständigt. Eine Geschichte ist »[...] an oral or written performance involving two or more people interpreting past or anticipated experience «(Boje I995: I000). Geschichten sind soziale Konfigurationsmomente, auf die mündlich oder schriftlich sowie materiell oder symbolisch Bezug genommen wird, die das beschreiben, was zu erwarten ist. Auf diese Weise wird Storytelling zu einem dreifachen »Multitool«, das Organisationskultur, Wissensmanagement und die Generierung von Stabilität verwaltet, Erfahrung und Kompetenzen (ab-)speichert und dieses auf praktische Weise vermittelt (Czarniawska 20I4b; Patriotta 2003). 
Czarniawska (20I4b) unterscheidet zwischen Storytelling als Kommunikationsmittel oder als Marketinginstrument. ${ }^{67}$ Durch Kommunikation werden Storytelling und Geschichten verfestigt und vermitteln ein »feeling of belonging« (ebd.: 73) bzw. führen zu was Czarniawska mit »organizing stories« konkretisiert, die als organisationsinterne Narrative für Externe nur schwer verständlich sind (2015: 83). Geschichten als Kommunikationsmittel und Instrument des Wissenstransfers ermöglichen Sinngestaltung und Bedeutungszuschreibung und fungieren als modus operandi, vor allem dann, wenn es um das Lösen bestehender Ungereimtheiten geht (Patriotta 2003). ${ }^{68}$

Organisierende Geschichten können zu organisationalen Geschichten werden. Werden organisierende Narrative $\mathrm{zu}$ einem nach außen gerichteten Sprachspiel gebündelt, können die organisationalen Geschichten als Marketinginstrument eingesetzt werden. Wichtig ist, dass definierte Geschichten grundsätzlich aus narrativen Fragmenten bestehen und konstruiert werden (Mützel 20I7: 66). Ohne Aneinanderreihung fehlt den einzelnen Narrativen ein übergreifender Sinn. Mittels »emplotment« wird aus narrativen Fragmenten eine Geschichte strukturiert und eine thematische Pointe oder ein sinnstiftender Plot modelliert (Czarniawska I998: 2 f. 20I5). ${ }^{69}$ Dieser Vorgang erleichtert die Aufbereitung und Außendarstellung der Geschichte und findet in der Modekreation sowohl vorgängig und während der Entwicklung und Produktion - in Form von Neuerungsnarrativen - als auch retrospektiv - dann als Kreativitätszeremonien - statt. Deshalb bezeichnen Lounsbury und Glynn diese Geschichten auch als »impression management stories«, die absichtlich ausgedacht und geplant werden (Lounsbury/Glynn 200I: 55I). Auswärts ge-

67 | Sie führt, drittens, die Verwendung des Storytellings als "managerial tool" aus, was ihrer Meinung nach eine defizitäre Beschreibung von Storytelling ist, weshalb aus diesem Konzept keine einschlägige Erkenntnis gewonnen werden kann. Storytelling kann Organisationen zwar erhalten, aber nicht erschaffen (2014b: 72).

68 Hier verbinden sich Problemlösen mit Geschichten zu einem sinnstiftenden Prozess. Patriotta spricht von Detektivgeschichten: "Problem solving takes the form of an inquiry in which the detective attempts to collect clues and reconstruct the fact. It implies locating anomalies in space and time, formulating hypotheses and conjectures, conducting interviews" (Patriotta 2003: 363). Demzufolge stellen Detektivgeschichten eine narrative Struktur und Handlungsanleitungen bei störenden Ereignissen bereit.

69 | Wird zur Erzählung zusätzlich eine Pointe oder ein Argument ergänzt oder in chronische Reihenfolge gebracht, wird aus einem Narrativ eine "story" (Polletta et al., 2011: 112). Anders verhält es sich gemäß Boje (1995 und 2001), der mit "stories" die Vorstufe der "narratives" versteht, welchen die strukturelle Gliederung (Anfang, Mittelteil, Abschluss) fehlt. Unfertige "story"-Fragmente (von Boje auch als "antenarratives" bezeichnet) gehen Geschichten (narratives) voraus. 
richtete Rhetorik kann zu mehr Legitimität führen (Lounsbury/Glynn 20or; Suddaby/Greenwood 2005; Petkova 2016). Vor allem, wenn Geschichten auf politische und gesellschaftliche Bedürfnisse reagieren, Modelle imitieren und Moden folgen, diffundieren sie erfolgreich (Czarniawska/Joerges I996: I7). Geschichten werden als Quasiobjekte verstanden, die immer wieder eingebettet und materialisiert und so - lokal interpretiert und subjektiv erzählt - zu intersubjektiven Objekten werden (Czarniawska/Joerges 1996: 23; Rhodes/ Brown 2005: I72). Die Verbreitung ist das eigentliche Ziel des Geschichtenerzählens, denn sie sind »communicated images, intersubjective creations, and therefore a sproperty< of a community rather than a single person « (Czarniawska/Joerges I996: 33, Hervorh. im Orig.). Das Werk aus fragmentierten Erzählteilen, das sich aus einer »pluralistic construction of a multiplicity of stories, storytellers, and story performance events « zusammenstellt, dient als narrative Grundlage der Darstellungsformen (Boje I995: I000). Zusammenfassend meint Storytelling das Erzählen von organisationalen, kollektiven, vielstimmigen, alltäglichen und spielerischen Fragmenten zum Zweck interner oder externer Kommunikation. Der kommunikativen und zeremoniellen Darstellung kreativer Idealisierung widmen sich die folgenden Kapitel.

\subsection{Orientierende Narrative}

$\mathrm{Zu}$ Beginn der Kollektion steht der Entwurf. Seine orientierenden narrativen Fragmente, d. h. das Thema und der Arbeitstitel, Modellnamen und Farbbezeichnungen sowie Fertigungsdetails, Kollektionslogo und Stoffdrucke, werden auf konzeptuelle, materielle oder symbolische Weise zu neuen Kreationen ausgearbeitet. Demzufolge orientieren diese Fragmente die Kommunikation und Darstellung und leiten die Kollektionshervorbringung an.

»Wir sind an dem Punkt, wo wir seit etwa drei, vier Jahren neue Kunden haben, denen wir etwas von unserer Seidengeschichte erzählen wollen«, sagt die Designerin, während sie zum ersten Mal das handlungsführende Konzept und die neuen Entwürfe im Designteam vorstellt. Die narrative Ausdrucksweise wird nicht erst nach der Entwicklung und Produktion der neuen Kollektion relevant. So bezeichnet die Designerin das Traditionsunternehmen als Maison, was in Anlehnung an die Tradition der französischen Mode- und Schneiderkunst verstanden werden kann. Damit wird ein Selbstverständnis vermittelt, das an die luxuriösen Couture-Häuser erinnert. Die Designerin des Strickwarenherstellers hingegen verortet sich bei den new basics, die sich im Grundschnitt an reduzierten (Herren-)Kleiderschnitten orientieren. Diese Bezeichnungen sind Narrative der Selbstbetitelung und reklamieren, wie die Unternehmen sich selber wahrnehmen und beschreiben. Narrative zum künstlerisch-kreativen Selbstverständnis der Creative Directors spielen auch 
in Bezug auf die technologische Umsetzung der Entwürfe eine wesentliche Rolle (vgl. Kap. 6.2.I).

Die Analyse des Feldmaterials hat eine Vielzahl von narrativen Fragmenten zum Vorschein gebracht, aus welchen im Verlauf des Entwicklungsprozesses allmählich eine Geschichte entsteht. Narrative Fragmente sind das Thema, der Arbeitstitel, das Kollektionslogo, Stoffdrucke, Modellnamen und Farbbezeichnungen. Es sind Markierungen, welche die Neuerungen in der Kollektion symbolisch und materiell fixieren. Ein Hinweis, dass Geschichten antizipiert und vorgängig, die nicht erst ex post als rhetorische (Marketing-)Strategie, sondern auch als vorgängige, orientierende Handlungsanleitung prospektiv bedeutend sind, zeigt das Beispiel der persönlichen Einladung zu einem Fotoshooting:

"Hast du Lust am Donnerstag zum Shooting zu kommen? Die Geschichte dahinter lautet folgendermaßen: Wir bringen den Schweizer Leinen vom Land in die Stadt - mit dem Velo! Wir fotografieren die Schweizer Flachs Kollektion. Eine kleine Linie mit 15 Modellen, die ganz in der Schweiz hergestellt wird. Um dies sichtbar zu machen, planen wir den Weg von Willadingen im Emmental, wo der Flachs wächst, in die Stadt - und das per Velo!"

Narrative Fragmente sind in Form des Themas, das sich weiter im Arbeitstitel manifestiert, von Beginn wesentlich an der Entwicklung der Neuerungsnarrative beteiligt und nehmen im Kollektionsverlauf materielle und symbolische Formen an. Eine vorgängig entstandene Geschichte wird zur handlungsdefinierenden Grundlage für die Ausgestaltung in Shows und das finalisierende Storytelling. So konnte während eines Feldaufenthalts beobachtet werden, wie die Projektteilnehmer während eines Shootings wiederholt das Narrativ in der Inszenierung suchten. Empirisch ist auffällig, dass narrative Fragmente sowie antizipierte Geschichten eine wesentliche Rolle in der Darstellung und Konstruktion von Kreativität spielen.

\subsubsection{Das Thema und der Arbeitstitel}

Das Thema ist die Grundlage der Kollektionsentwicklung, die anschließend seriell produziert wird. Dabei dient der Arbeitstitel der kollektiven Orientierung. Das Thema wird vom Designer festgelegt und in der ersten Vorstellung der neuen Entwürfe bekannt gegeben. Anschließend wird es auf unterschiedliche - symbolische ( $z$. B. Arbeitstitel, Farb-, Modellnamen) und materielle (vestimentäre Fertigungsdetails, Accessoires, Stoffdruckmuster) - Weise in die Kleidermodelle eingearbeitet. Weiter trägt es zur narrativen und zeremoniellen Gestaltung der Kollektion bei. Auf der Grundlage des Themas wird anschließend durch inszenierende Shows (in Modenschauen oder im Fotoshooting) und durch finalisierendes Storytelling (z. B. in Newslettern oder in Kollektions- 
broschüren) ein kohärentes Narrativ zu einem Plot zusammengefügt, das der glaubhaften und authentischen Darstellung einer vermeintlich geplanten Geschichte dient. Als Folge zieht sich Thema als roter Faden durch die Kollektion und unterscheidet sie von eigenen vergangenen wie auch von Kollektionen der Konkurrenz. Das Thema markiert Erneuerungen und Veränderungen.

Woher stammen diese Geschichten? Aus dem Interview mit der Designerin und den Feldnotizen geht hervor, dass Ideen für Themen von Museumsund Archivbesuchen oder aus Literatur (Abb. 4) oder alläglichen Inspirationen (Abb. 5) herstammen:

Diese Ideen kommen von Geschichten, die sie in ihrem Kopf hat. Manchmal stammen sie aus der Literatur, die sie gerade lese, Charles Bukowski oder Allen Ginsberg, oder aus Filmen, die sie neulich gesehen hat, beispielsweise Jack Kerouacs On the Road, oder Museen, die sie kürzlich besucht hat, wie das Bauhaus Archiv. Die Geschichten sind nur für sie wichtig; anderen erzählt sie diese nicht.

\section{Abb. 5: Kampagnenbild $\gg$ On the Road«}

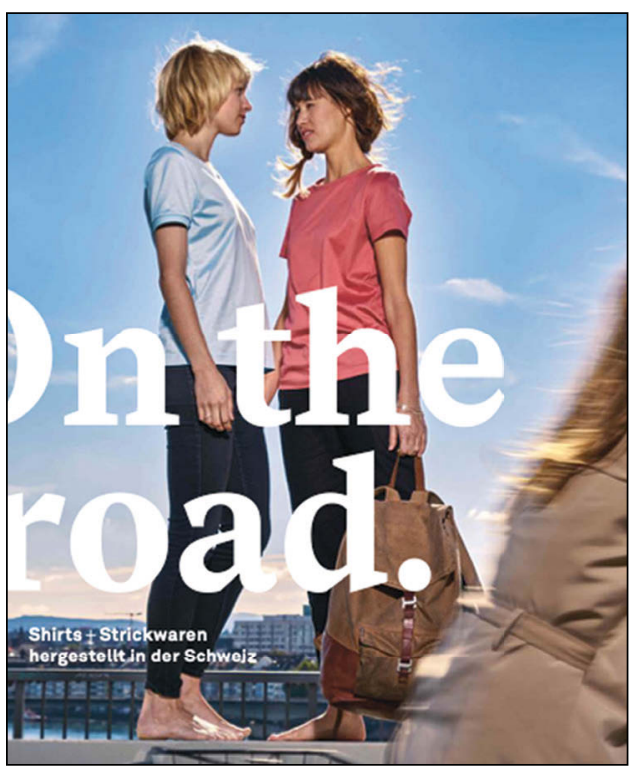

So hat sie zum Beispiel für die Herbst-Winterkollektion 2016/17 das Thema Instrumente ausgesucht und das sei sehr "zuvorkommend" gewesen, da viele Instrumente auf der Seite eine Verstärkung hätten. In Anlehnung daran hatte sie eine klare Vorstellung davon, wie sie diese mit Strick umsetzen wollte. 
Abb. 6: Kampagnenbild »Instrumente«

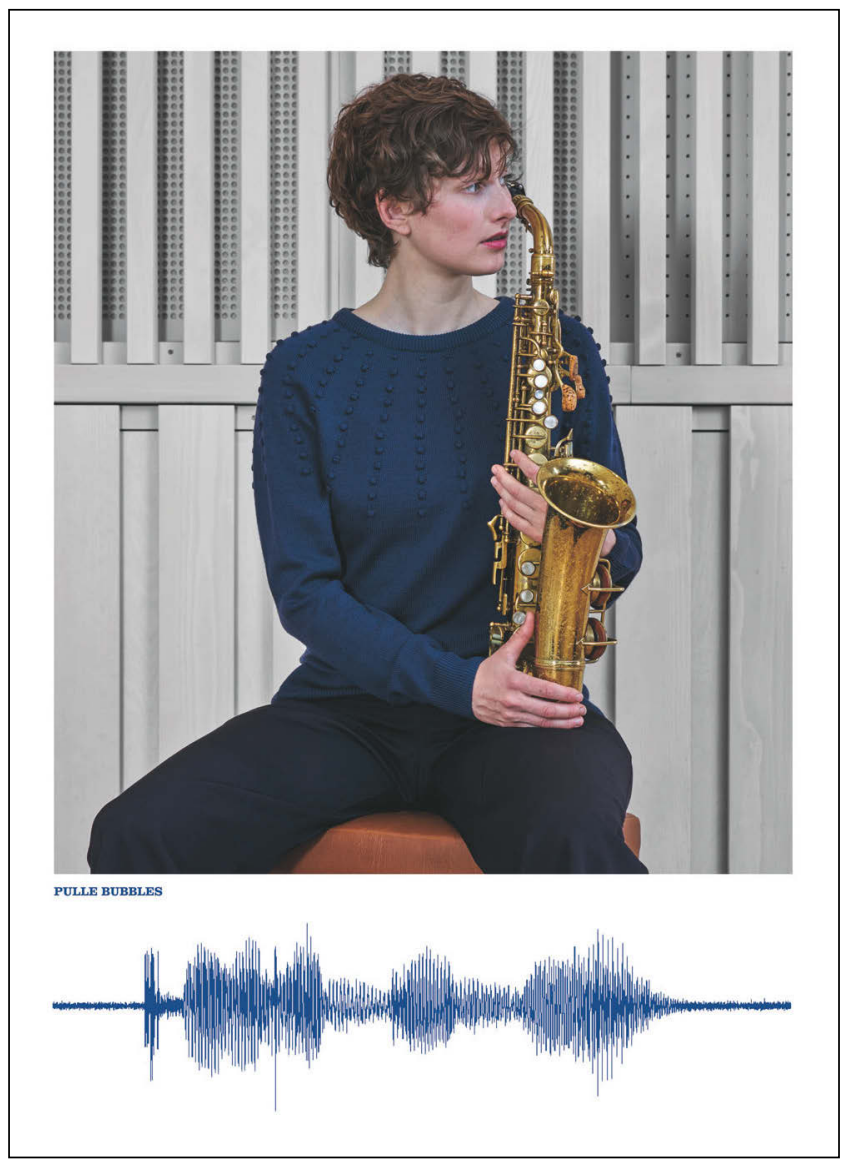

Das Thema dient als Referenzpunkt für den Plot - das Musizieren und die Darstellung der Instrumente - und schafft gleichzeitig einen Referenzrahmen für die technologische Umsetzung. Die Idee der Instrumente inspirierte nicht nur die Story des Fotoshootings, sondern auch die technologische Realisierung auf der Strickmaschine. So beschreibt die Designerin, wie sie, vom Thema ausgehend, den Schnitt definiert und dann daran arbeitet, dieses Thema als Stil durch die ganze Kollektion zu ziehen und herauszuarbeiten. Das Thema wirkt als textile Richtlinie, die die schneidertechnische Ausarbeitung der Kollektion bedingt. Insofern ist das Thema als narratives Fragment ein symbolisch-konzeptuelles Merkmal der neuen Kollektion, orientiert technologische Entscheidungen und kreative Ausgestaltungen, ist aber kaum relevant für die administrative Verwaltungstätigkeit. 
Narrative Fragmente können auch von der organisationalen Biografie stammen und sich auf designnahe Themen beziehen (Abb. 6). Im Gespräch zwischen Schneiderin und Designerin wird der thematische Bezug geklärt:

"Weißt du, was das Konzept der Kollektion ist?" Die Designerin informiert die Couture-Schneiderin über die aktuelle Kollektion. Die Winter-Kollektion 2017/2018 dreht sich um alle Berufe, die im Gebäude des Modeunternehmens vorkommen. Das Ziel dabei ist, den Kunden von den verschiedenen Berufsbildern zu erzählen. Auf einer Skizze sind Figuren mit Berufsbezeichnungen betitelt in unterschiedlichen Kleidern zu sehen. La directrice, la céramique, la couturière, la vendeuse, la joaillereuse ...

Abb. 7: Skizze der Kollektionsmodelle und Berufe im Hauptgebäude

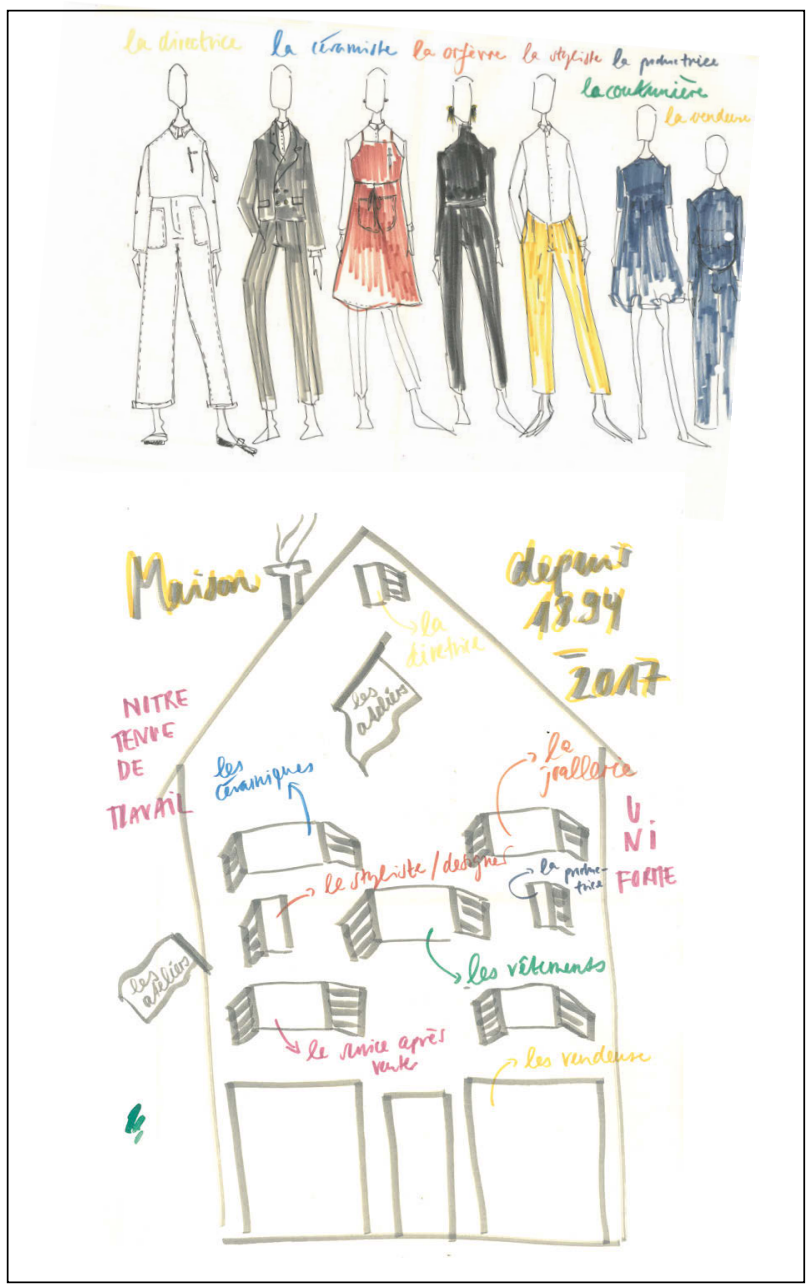


Aber auch produktionsnahe Angelegenheiten werden zu Themen und als solche zur Grundlage für die Ausgestaltung neuer Modelle (Abb. 7):

Mit der Herbst-Winterkollektion 2016/17 will die Designerin über die eigene Seidengeschichte erzählen. Sie will darauf aufmerksam machen, dass die Stoffe nicht nur exklusiv für sie gedruckt, sondern auch gewoben werden. Während ältere Kollektionen über unternehmerische Expansion in die USA oder Indien informierten, soll diesmal über die interne, exklusive Entwicklung und Produktion berichtet werden. Die Kollektion wird als Gefäß für Mitteilungen bezeichnet.

Abb. 8: Kampagnenbild »La Route de la Soie«

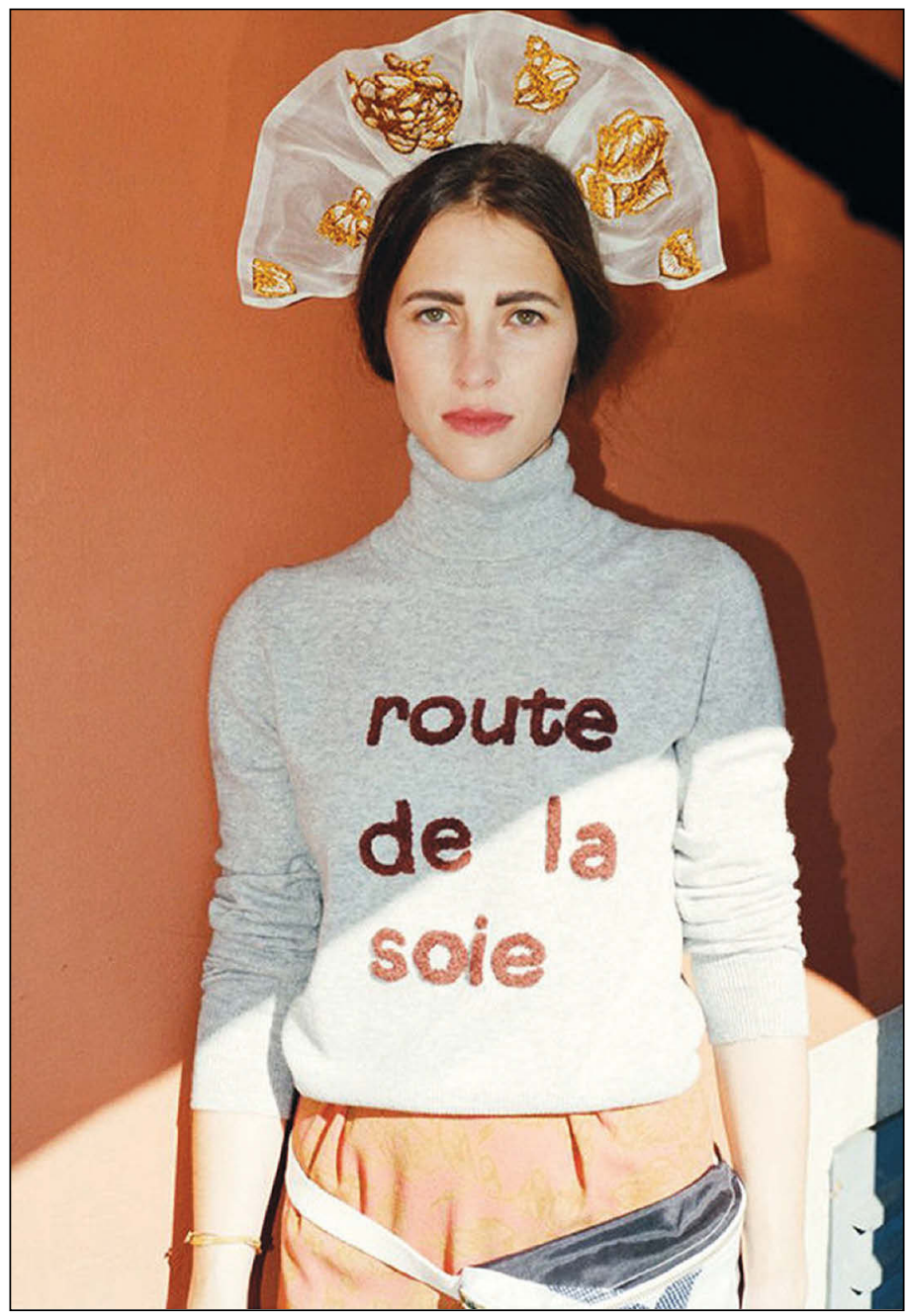


Diese Beispiele illustrieren, dass Themen - auf welche die Arbeitstitel folgen sowohl Momente der Biografie der Modeunternehmen als auch aktuelle oder besondere Interessen der Designer umfassen. Damit beleuchten sie verschiedene Facetten des Unternehmens. Weiter fungiert das Thema - ähnlich den Prototypen oder Analogien - als Kommunikationsmedium zwischen temporären Projektmitarbeitern und permanentem Personal. »Kennst du das Thema?«, wurde oft gefragt, wenn ein zusätzliches Projektmitglied über die aktuelle Kollektion informiert werden sollte. Diese Frage unterstreicht sowohl die thematische Bedeutung für den weiteren Entwicklungsprozess als auch für das Verständnis der Kollektion.

Das Thema und sein Arbeitstitel sind narrative Fragmente, die zum Grundstein des Plots werden, der retrospektiv aufbereitet und als Geschichte zur Kollektion erzählt wird. Auf diese Art werden die narrativen Fragmente Teil der Neuerungsnarrative der Kollektion. Als konzeptuelle Grundlage verfestigt sich das Thema in den Phasen der Kollektionsentwicklung in textilen und materiellen Hinweisen und ist somit gleichzeitig künstlerisches Beiwerk der Handlungsroutine der Herstellung von neuen Kreationen.

\subsubsection{Materielle Markierungen: Logo, Stoffdrucke und Fertigungsdetails}

Da der Arbeitstitel nur vorläufig besteht, wird das narrative Fragment Titel in der materiellen Ausarbeitung der Kollektionsteile sowie der dazugehörigen textilen Details ausgedrückt. Materielle, narrative Fragmente sind das Kollektionslogo, die Stoffdrucke oder die vestimentären Fertigungsdetails. Das Logo (Abb. 8) ist eine materielle Referenz, welche die Kollektionsteile zu einer Einheit verbindet und die Narrative materialisiert. Im Feld wird die Bedeutung erläutert:

Der Arbeitstitel hat den Namen der Familienfarm in Argentinien, auf welcher die ganze Familie Weihnachten und Neujahr verbracht hat. Sie hat ein Auge mit einer Träne gezeichnet. Das Bild wird das Logo der Kollektion. Die Träne und das Auge dürfen aber nicht allzu traurig wirken, deshalb macht sie einen lachenden Mund dazu. 
Abb. 9: Kollektionslogo

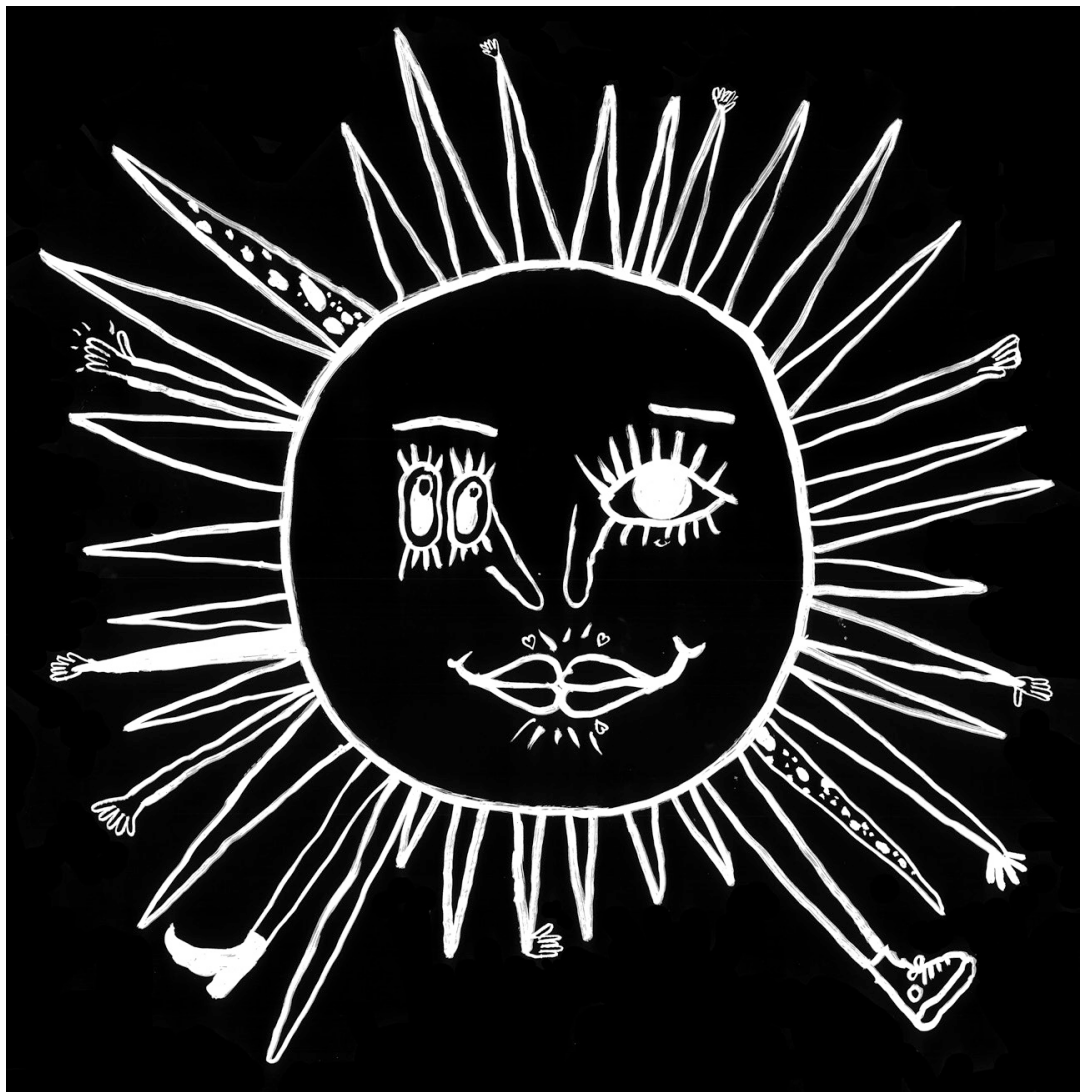

Die Idee, die sich nun im Arbeitstitel spiegelt, wird in einem kollektionsspezifischen Stoffdruck interpretiert. Der Titel wird mit dem Fortgang der Kollektionsentwicklung in Form des textilen Logos materialisiert.

Und auch kollektionsspezifische Stoffdrucke werden zu Trägern des Themas gemacht. So wird in der Vorstellung neuer Kollektionsentwürfe das Muster eines Schals, der sich über mehrere Jahre als Klassiker im steten Bestand wechselnder Kollektionen bewährt hat, nun zu einem thematisch passenderen Druck abgeändert:

Die Designerin kommt zum Print. Seit 20 Jahren haben sie den "Leo-Dot", den sie nun zum hauseigenen Punktemuster ändern will. Das künftige gepunktete Muster soll eher wie ein Luchsmuster aussehen, denn der Luchs ist ein einheimisches Tier und das Muster etwas feiner. 
Das Leopardenmuster wird zum Luchsmuster umdesignt und dieses zum Symbol für die lokale Verwurzelung. Aus der Interpretation und den Weiterentwicklungen bestehender Elemente bilden sich Muster, die dann später wieder aufgegriffen und absichtlich zurückgewiesen oder variiert werden.

Darüber hinaus werden textile Markierungen in Berücksichtigung der ursprünglichen Idee gefertigt, um materielle Hinweise zum Thema zu schaffen. Ein Beispiel sind Fertigungsdetails (so z. B. Manschette, Kragen, Schnallen, Knöpfe, Reißverschlüsse, Label und die dazugehörigen Farben), die vermehrt als Neuerungen der bestehenden Modelle fungieren; also wandelbare stilprägende Elemente, die angepasst werden und das Modell deshalb nur minimal verändern. Die Designerin reflektiert über diese Entscheidungen:

Die Designerin macht sich zur Sommerkollektion 2017 Gedanken und überlegt sich, welche Klassikerteile bleiben und welche Details (z. B. Manschetten oder Kragen) sich verändern sollen, ebenso wie diese Veränderung aussehen soll. Sie nennt dies die stilprägenden Elemente. [...] Sie überlegt, an der Hose eine kleine Schnalle einzubauen, damit man sie enger machen kann. Gerade zeichnet sie an einem Band, das so auf der Seite an die Hose genäht wird. Die Hose, an der sie arbeitet, ist eine Argentinische Reiterhose, die sie aber nicht genau so übernehmen kann. Sie übernimmt das Riemchen als Element und verwendet es für ein Hosenmodell, das sie selbst entworfen hat.

Ein weiteres Beispiel davon ist die Erläuterung der Designerin, die mehr Zeit und Kreativität in ein bestimmtes Detail an einem Kleid investieren und so die Zugehörigkeit des Kleides zum Thema der Kollektion verdeutlichen will. Diese Verdeutlichung soll in Form einer kleinen Tasche für das Messband oder als darauf gestickte kleine Schere zum Ausdruck kommen. Ob und wie solche vestimentären Details erschaffen werden, wird gemeinsam besprochen, wobei wieder die technologische Realisierbarkeit wegweisend ist. Demgegenüber wird das grundlegende Thema vom Projektteam unhinterfragt aufgegriffen und zur Richtschnur des Projektauftrags. Materielle Markierungen sind stärker von Technologien abhängig als symbolische.

\subsubsection{Symbolische Markierungen: Namen und Bezeichnungen}

Symbolische, narrative Fragmente sind Modellnamen oder Farbbezeichnungen. Allen Kollektionsteilen werden Namen zugewiesen, die auf das übergeordnete Thema und den Arbeitstitel verweisen und die einzelnen Kollektionsmodelle durch die thematische Rahmung zu einer Einheit - der Kollektion - verbinden. Entsprechend wird die Namensgebung der Modelle zum Inhalt der Vorstellungssitzung neuer Kollektionen: 
Die Namen für die neuen Modelle sind populäre argentinische Namen aus dem Jahre 2014: das überarbeitete Serafin-Kleid heißt nun Alma, das Hemd Lola, die Hose Benjamin, der Blazer Joaquin und der Name Mia wird, da es inn schon mal gab, mit Maddalena ersetzt. So lautet der Name der Nanny ihrer Tochter. Das sei zwar portugiesisch, gäbe es aber sicher auch in Argentinien.

Die Modellnamen sind symbolisch-sprachliche Hinweise und Bestandteil des Neuerungsnarrativ, welches die Geschichte später abrundet und die einzelnen Modelle sprachlich zu einer Einheit verbindet.

Ebenso wie die Modellnamen werden auch die Farbbezeichnungen sorgfältig in Übereinstimmung mit der Geschichte ausgewählt und zu sprachlichen Hinweisen kuratiert. Während einmal die Namensvergabe der neuen Modelle im Zuständigkeitsbereich der Produktion liegt, sind woanders die Farbbezeichnung und die Zusammenstellung eines Farbkonzeptes Aufgabe des Designers. Die Farben Green Sixties, Zora, Aube, Snapdragon oder Blue Graphit verstehen sich als Indizien der Lavalampen aus den i97oern oder stellen mit Latte di pistacchio, Sorbetti di fragola, Puffo oder Fiore delle [sic!] mandorle sprachliche Verbindungen zur italienischen Eiskultur her und symbolisieren die Neuerungen der neuen Sommerkollektion. Das folgende Beispiel stammt aus einem Newsletter und zeigt die ungewöhnliche Sprache, mit welcher die neue Farbpalette beschrieben wird.

Einen frischen Anstrich aus "Herbst-Rhythmen" - ein Mixtum compositum aus "SONIC «-Künstlern wie dem Rhapsody in Blue-virtuosen Gershwin, Soul Surfer Antony \& The Johnsons oder etwas rosa [sic!] aus Billie Holidays Garderobe. Aneinandergereiht und angestoßen von Paul Klees Bild Hammamet geben diese den Winterton an.

Die beiden Beispiele zeigen, wie das Unternehmen mittels sprachlicher Darstellung, d. h. der Verwendung besonderer Vokabulare, Kreativität konstruiert und damit die Erneuerung und Einzigartigkeit der Kollektion kommuniziert.

Vom Anfang in der ursprünglichen thematischen Idee, die in der ersten Kollektionssitzung vorgestellt wird, manifestiert sich ein roter Faden, der die narrativen Darstellungen anleitet und entlang dessen sie sich entfalten: Vom Thema und dem Arbeitstitel zu stilistischen, materiellen Elementen wie Logo, Stoffdrucke und Fertigungsdetails hin zu symbolischen Elementen der Bezeichnungen neuer Modelle und Farben entsteht allmählich eine aus narrativen Fragmenten komplettierte Geschichte. 


\subsection{INSZENIERENDE ShOWS}

Nachdem der Entwurfsidee durch das Thema materiell und symbolisch Ausdruck verliehen wurde, werden die Neuerungsnarrative nun rhetorisch und spielerisch aufbereitet, inszeniert und zur Schau gestellt. Modenschauen und Fotoshootings sind Darstellungsformen der Mode, die die neuen Kreationen zur Schau stellen. Sie sind ressourcenintensiv und als Plattformen kreativer Zurschaustellung ein unverkennbarer Bestandteil der Modeindustrie. Die inszenierten (und inszenierenden!) Darstellungsformen beinhalten ein Konzept, die Zusammenstellung der Kleidung, Accessoires und Models und schließlich einen oder mehrere Schauplätze. Ressourcenintensiv sind Schauen, weil zusätzliche Materialien gebraucht werden, wie z. B. Accessoires, ein oder mehrere Schauplätze und mehr Personal, denn die Kleider werden von lebendigen Mannequins getragen. »Was ist hier das Narrativ?«, ist die zentrale Frage dieser Phase, welche die Projektmitglieder wiederholt stellen und die sich als roter Faden durch die einzelnen Einstellungen der Szenen (im Fotoshooting) oder unterschiedlichen Outfits respektive Kollektionen (in der Modenschau) zieht und stets an das ursprüngliche Thema erinnert. Diese inszenierenden Darstellungsformen haben ein zeremonielles Moment, das ihr Ausbleiben undenkbar macht.

\subsubsection{Das Konzept}

$\mathrm{Zu}$ Beginn erarbeitet der Creative Director, manchmal in Zusammenarbeit mit anderen Projektmitgliedern wie dem PR-Manager, dem Grafiker oder dem Fotografen, ein Konzept aus, das Themen in Erinnerung ruft, den Plot der Geschichte festlegt und zum Ziel hat, diese zu transportieren. Dieses Konzept ist die orientierende Arbeitsgrundlage für die Inszenierung. Darin sind Anleitungen für Licht/Schatten, Muster/Farben, Posen/Bewegungen und Objekte/Accessoires enthalten, sodass der Plot für das Publikum sichtbar gemacht wird. Eine Feldnotiz hält fest:

Die Konzepte beinhalten die Kleiderkombinationen, die maximale Zahl der Aufnahmen, sie beschreiben den Hinter- und Vordergrund und wie das Model posieren soll. Zur Orientierung für die gesamte Idee und Komposition des Models im Bild werden zusätzlich Inspirationsbilder mitgegeben.

Das Konzept fungiert wie eine Landkarte, auf welcher der aktuelle Standort sowie die vorgegebenen Ziele ausformuliert wurden, d. h. die Tagesziele des Fotoshootings und der Modenschau. Folglich bündeln Konzepte den Blick der Akteure auf den Arbeitsauftrag und geben, z. B. mit Inspirationsbildern, praktische Anweisungen dazu. Das Konzept hält fest, wie die Kleidung den Raum 
aufnehmen und das Licht spiegeln soll; wie die Farbigkeit gestaltet und in welcher Reihenfolge sie präsentiert respektive in welchen Räumen sie fotografiert werden soll. Im Feld kann beobachtet werden, wie die Designerin sich an dieser Handlungsanleitung ausrichtet:

Die Designerin fragt, ob das weiße Kleid nicht außerhalb des Farbkonzepts liegt? Wenn der Fotograf die Treppe nicht frontal, sondern leicht schräg fotografiert, dann wären mehr Weißtöne auf dem Ausschnitt und das Bild würde besser ins Konzept passen.

Die Projektgruppe arbeitet nahe am Konzept und versucht, die entwickelte Vorlage umzusetzen. Diese gilt während der Aufnahmearbeiten als Richtschnur für das Licht, die Komposition, die Posen und die Aufnahmetechnik, anhand derer versucht wird, der Kollektionsdarstellung eine einheitliche Optik zu verleihen. So sollen die Kleider, die Räume und die Bildausschnitte miteinander korrespondieren und sich zu einer passenden Bildsprache bzw. der Darstellung der Geschichte zusammenfügen.

Auch für die Modenschau wird ein Konzept entwickelt, das die unterschiedlichen Präsentationen der teilnehmenden Modefirmen reguliert und dadurch teilweise vereinheitlicht. Das Konzept definiert die Möglichkeiten der Kleiderkombinationen, es kontrolliert die inhaltliche Zusammenstellung der Looks und die Präsentationsreihenfolge. Hier wird das Konzept vom Modenschauveranstalter entworfen. Zusammenfassend koordinieren Konzepte sowohl die projektinterne Kommunikation als auch die einheitliche Kollektionsdarstellung.

\subsubsection{Das Outfit}

Für die visuelle Ausgestaltung werden die Kleiderkombinationen mit Accessoires ergänzt und von Models präsentiert. Ausgewählte Looks (meistens sind es I3) der gesamten, fertigen Musterkollektion werden zu einer Modenschauauswahl zusammengestellt. Deshalb wird die Musterkollektion auch als Peak der Kreativität bezeichnet, denn die strengen Selektionskriterien haben dazu geführt, dass nur noch Muster hängen, die vom Designteam als besonders kreativ bewertet wurden.

Das Zusammenstellen der Kleiderkombinationen erfolgt in einer eingehenden Auseinandersetzung und in intensiven Arbeitsphasen, die sich unregelmäßig wiederholen, bis der Designer zufrieden mit der Auswahl ist. Die Outfits werden dann mit Accessoires ergänzt und zu Looks oder Key Looks zusammengestellt, die nicht selten mit Show Pieces vervollständigt werden, und in/an einer bestimmten Location vorgeführt. Im Feld ist dieser Prozess auch als Styling bekannt. Looks bestehen aus den selektierten Kollektionsteilen, die 
manchmal auch Show Pieces - Kleidung oder Accessoires, die entweder nur zu Showzwecken hergestellt oder aus ökonomischen Gründen später nicht seriell produziert werden -, zusätzlichen Accessoires und dem Model, dessen Frisur und Schminke. Die Zusammenstellung von Kleidung, Models und Accessoires wird sorgfältig orchestriert. Die Designer planen dafür besondere Zeit ein und sind dabei hoch konzentriert, weshalb sie den Eindruck eines Kurators machen, der sich der Umsetzung einer Ausstellung widmet. Eine Beobachtungsnotiz beschreibt, wie die Zusammenstellung der Outfits sich an Stoffmaterialien und Farben orientiert.

Als erstes stellt die Designerin die Ober- und Unterteile zusammen und ergänzt sie mit passenden Schuhen, Hüten oder Taschen. Sie hat sich die Kombinationen der Kleider auf einer Liste notiert. Die Kleidung wird in die Reihenfolge des Aufnahmen-Ablaufs gelegt, so dass die Models die Kleidung sortiert der Reihe nach vorfinden und ohne zu überlegen so anziehen können.

Der Creative Director bereitet die Kleiderkombinationen vor und notiert oder fotografiert die geplanten Looks. Anhand dieser Fotos und Listen werden die Models vor Ort bei der Modenschau oder dem Fotoshooting im Fitting gekleidet. Die Planung des Kombinierens betrifft jedoch nicht nur die Kleidung und mögliche Accessoires, sondern auch die Wahl des Models, welches das Outfit vorführt, und den Ort, an dem die Inszenierung stattfinden soll. So informiert die Designerin über das anstehende Fotoshooting: »Ein extravagantes Shooting steht an: im Jazzcampus performen Io Jazzstudenten und ihre Instrumente in der neuen Winterkollektion « (persönliche Korrespondenz, 09. 05. 20I6). So wird die Jazzschule zum Ort des Geschehens, wo das Narrativ zwischen Kleidung, dem Thema Instrumente und den Musikern als Models eine Verbindung herstellt.

Das Vorgehen wird zeremoniell wiederholt: Die Kollektionsteile werden mit den Accessoires und den Modelporträts wie Puzzleteile hin und hergeschoben, bis die richtige Kombination steht..$^{\circ}$ Um Perfektion zu erlangen, beansprucht dieser Prozess mehrere Tage, wobei die Kombinationen im Designteam genau durchgesehen, diskutiert, korrigiert und angepasst - selten: belassen - werden.

70 | Das ist ein Beispiel interner Kreativität: Der Designer fokussiert auf die Kleidung, die Erscheinung und nicht die Art und Weise, wie diese zusammengestellt werden. Deshalb verweist die einzelne Kleiderkombination auf die zugrunde liegende Geschichte der Kollektion und ignoriert die Belange und Herausforderungen des Tüftelns; hier der kombinatorischen Versuche der Modelle, der Accessoires, der Schminke und Frisur und dem Model, welches die Kombination schließlich präsentieren wird. Diese Anstrengungen bleiben unsichtbar und werden nicht zur Handlung der Geschichte für die allgemeine Öffentlichkeit. 
Das intuitive Zusammenstellen der Kleider beginnt in der Entwicklungsphase während der Musterungen, in denen die Designer oder die Produktionsmanager $\mathrm{ab}$ und $\mathrm{zu}$ Kleider anziehen und innerhalb der Kollektion kombinieren und so Kombinationsvorschläge entwickeln. Später finalisiert das Styling die Kleiderkombinationen für die Modenschau und das Fotoshooting. Das Styling meint die Komplettierung der Looks durch Accessoires, das präsentierende Model (welches durch ein Fotoporträt vertreten ist) sowie der Schminke und der Frisur. Manchmal wird für das Styling eine externe Stylistin beigezogen. Diese Arbeit vermittelt den Eindruck einer äußerst wichtigen Aufgabe, die deshalb akkurat präpariert und sorgsam kuratiert wird. Diese Präparation verbindet die einzelnen Kleider und stellt einen übergreifenden, kreativen Sinn unter ihnen her.

Schließlich wird dieser Vorgang im Fitting, der Hauptprobe vor der Modenschau, wiederholt und die Kleiderkombinationen, die Accessoires und die Models werden ein letztes Mal miteinander abgeglichen. In diesem Moment werden Look-Komponenten (Model, Kleidung, Accessoires) noch einmal überprüft und allenfalls kurzfristig geändert. Auch diese Entwicklung der definitiven Zusammenstellung des Outfits ist eine zeitintensive Aufgabe, wie eine Beobachtungssequenz zeigt:

Jeder Look wird einzeln an den Models ,durchprobiert. Der Modenschau-Organisator erlaubt 13 Looks; manchmal kann die Auswahl um einen zusätzlichen ergänzt werden. Obwohl die Designerin genügend Auswahl dabeihat, ist sie nicht ganz zufrieden mit den Kombinationen. Sie stört sich an den Kleider- und Model-Kombinationen. Die Models in den Kleidern wirken etwas schlapp und schludrig und die Designerin erklärt, dass ihre Kleider dieses Unseriöse nicht vertragen; dass der Look dann wie unpassend verkleidet aussieht. Entweder werden die Kleider oder die Models ausgetauscht oder ersetzt, oder die Modenschau-Auswahl wird um einen Look gekürzt.

Hier öffnet sich das Projektteam und die Einschätzungen aller Projektteilnehmer werden herangezogen. Einmal kann beobachtet werden, wie sogar Vorschläge der Models oder der Ethnografin angehört und berücksichtigt werden. Das Fitting spielt beim Fotoshooting keine Rolle; es gibt keine Hauptprobe, da spontan auf Veränderungen und andere Kombinationen reagiert werden kann.

Schließlich wird aus allen möglichen Looks einer ausgewählt und zum ikonischen Key Look ernannt. Der Key Look ist eine ausgewählte Kleiderkombination oder eine fotografische Komposition, die als ikonisches Sinnbild das Zentrum der neuen Kollektion festlegt. Sowohl in der Modenschau als auch bei den Fotoaufnahmen spielt diese Schlüsselkombination eine wichtige Rolle. Zusätzliche Dokumente verweisen darauf: »Auf einer Postkarte, die im Showroom aufliegt, ist der Key Look zu sehen, an den man sich gerne erinnert; dieser Look soll das Publikum an die Modenschau und den Brand erinnern.« 
Der Key Look stellt das Thema auf besondere Weise dar und wird so komponiert, dass er das Narrativ der Kollektion bestmöglich repräsentiert.

Die Zuteilung von Kleidermodell, Model, Accessoires und Darstellungsort findet sowohl in Einzel- als auch in Teamarbeit (Creative Director in Zusammenarbeit mit dem PR-Manager, Produktionsmanager oder dem Stylisten) statt. In der heterogenen Projektkonstellation bedingt die Expertise den Fokus: Der Fotograf kommentiert den Lichteinfall oder den Schattenwurf der Falten auf dem Kleid, das Modell kommentiert, wenn sich eine verlangte Pose unnatürlich anfühlt, und der Designer sucht das passende Outfit für die ausgewählten Räumlichkeiten aus und bemerkt, wenn das Kleid nicht richtig fällt oder die Prototypen-Größe dem Model nicht passt.

\subsubsection{Der Schauplatz}

Immer wieder werden die Auswahl und die Bedeutung des Schauplatzes der Show angesprochen. Der Schauplatz spielt eine besondere Rolle im Konzept der Inszenierung der Kollektionsgeschichte. Dabei ist sowohl wichtig, wie Kreativität transportiert wird, als auch wo die Darstellung stattfindet. Während Fotografien auch in zu diesem Zweck eingerichteten Studios gemacht werden, werden nicht selten besondere Locations ausgesucht, die - wie am Beispiel der Jazzschule gezeigt - zur visuellen Darstellung der Kollektion beitragen. Gleichermaßen wird für die Modenschau ein Raum gewählt, der sich für die Präsentation von Mode eignet. Die Wahl des Schauplatzes ist Teil des Konzepts. Die Unternehmen suchen themennahe Örtlichkeiten für die Fotoshootings aus, so zum Beispiel das eigene Wohnhaus, um die Familiarität im Unternehmen zu betonen, die Straßen um das hiesige Geschäftslokal, um Stadtverbundenheit zu signalisieren, oder das spanische Klublokal für die Darstellung eines maritimen Looks, das in einer Feldnotiz ausführlich beschrieben wird (9):

Das Wandbild trägt zum Ambiente im Restaurant bei, das die Szene des maritimen Looks der Sommerkollektion 2015 prägt. Für die Kollektionsbroschüre wurden die Wandbilder mit den Fischen und das Model mit dem Matrosenhut zu einer maritimen Collage kombiniert. 
Abb. 10: Bildausschnitt des Fotoshootings im spanischen Klublokal

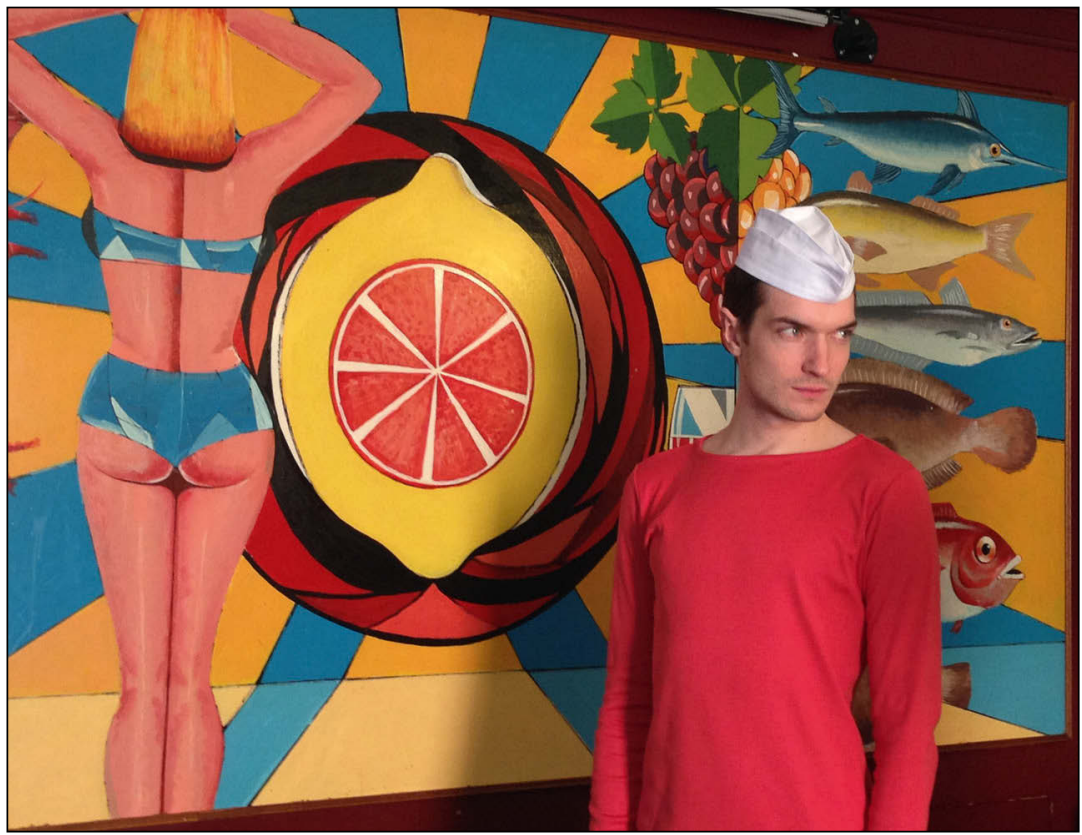

Die Lokalität trägt visuell zum Storytelling der Kollektion bei, indem es den Look optisch in eine Umgebung integriert und so eine eigene, typische Kollektionsästhetik entwickelt. Das Ambiente eines Kellerzimmers, das einer Abstellkammer gleichkommt, in der alte Matratzen und Golfschläger, eine Puppenhäusersammlung und einige leere Kartonschachteln herumstehen, wird vom Grafiker mit mad und spooky bewertet. Vor allem »das Esoterische« wird in den Szenen geschätzt, in denen die physischen Dinger - Puppenhäuser, aber auch andere Artefakte wie beispielsweise objets - selber leben, aber trotzdem als autonome Gegenstände behandelt werden. Die Aufnahmen sollen dabei unambitioniert und möglichst natürlich wirken. Über solche Vokabulare finden Bewertungen während der Aufnahmearbeiten statt.

Das Team entscheidet vor Ort am Schauplatz über die Zuteilung der Aufnahmeeinstellungen, Räume und Outfits. Diese Zuteilung wurde zwar vorgängig vom Designer geplant, nun aber vor Ort im Kollektiv konkretisiert und manchmal auch geändert. Die Wahl des Schauplatzes ist nie arbiträr, sondern folgt dem Thema der Geschichte, die zwar erst in der Postproduktion narrativ-visuell gefertigt wird, aber als Leitlinie schon während der Arbeit am Set präsent ist. 


\subsubsection{Die Accessoires}

Des Weiteren umfassen die Ausgestaltungen der Shows auch Accessoires, die die Inszenierung komplettieren und nicht selten zu diesem Zweck extra hergestellt werden. Accessoires sind Utensilien des inszenierenden Spiels, welche einen Teil der Geschichte symbolisieren oder hervorheben und so zum Storytelling beitragen. Sie decken eine Reichweite ab, die von illustrativen Objekten wie beispielsweise Sportgeräten (z. B. Badminton-Racket, American Football), über Objekte, die lediglich für die Modenschau produziert wurden - Show Pieces - (z. B. Schweizer Trachten-Kopfschmuck), bis hin zum Hund der Designerin, der während der Schau von den Models Gassi geführt wird. Ein Beispiel sind bemalte Schuhe, ein anderes sind die objets, die lediglich zum Zweck des Fotoshootings genäht wurden und deren Wichtigkeit in folgender Notiz zum Ausdruck kommt (Abb. Io):

Die in-house Couturière hat die Comic-Elemente, die als Logo des kollektionseigenen Musters den Stoff verzieren, in Kissenform genäht. Diese objets werden für das Fotoshooting gebraucht. [...] Entweder hält der Grafiker die objets selber, oder fixiert sie so, dass seine Hand nicht gesehen wird. Da er das Comic-Muster für die Kollektion ausgearbeitet hat, übernimmt er am Set nun die Aufgabe, die objets in der Fotografie zu platzieren.

Abb. 11: Kampagnenbilder mit Comic-Elementen

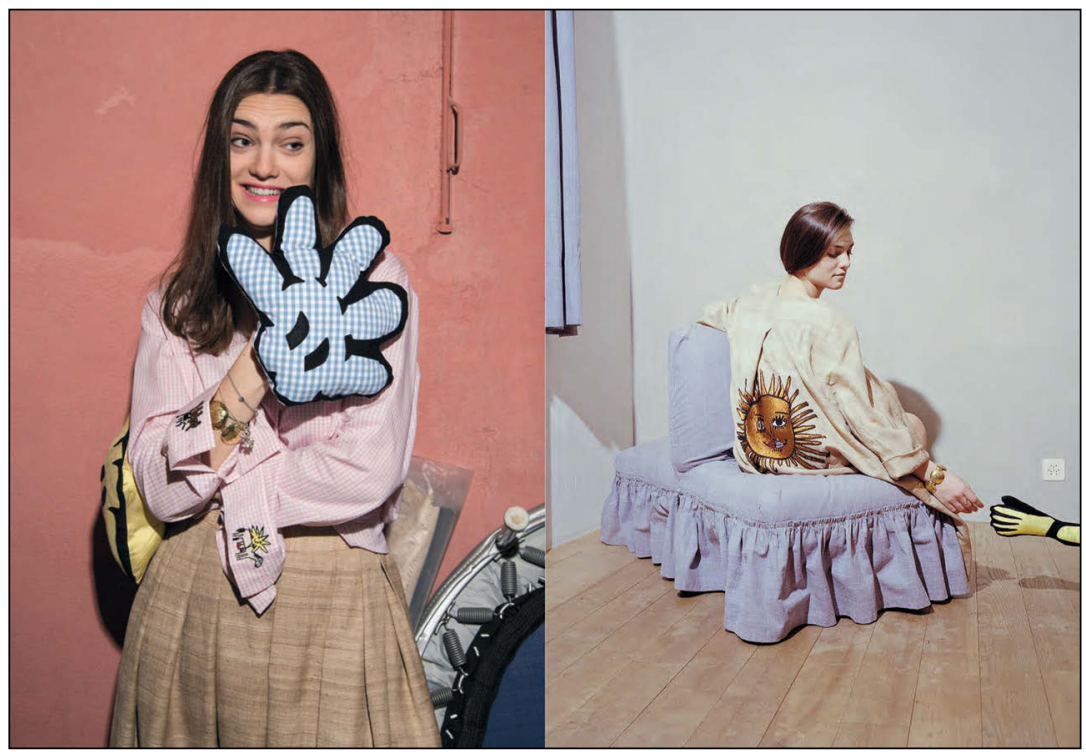


Illustrative, Show-spezifische und natürliche Gegenstände (und Lebewesen) sind zusätzliche Elemente, die der kreativen Inszenierung und zeremoniellen Darstellung der modischen Neuerungen dienen. So wird zum Beispiel eine Seidenhaube auf einem Haarreifen, die der Kopfbedeckung einer Innerschweizer Tracht ähnelt, ungefähr ein dutzend Mal hergestellt (Abb. 7). Die Accessoires sollen die Kleiderkombinationen mit einem Bruch versehen und einerseits Verwirrung stiften, andererseits aber nicht $z u$ viel sein. Ein solcher Bruch sind beispielsweise die mit Fensterfarbe selbst gestalteten Schuhe, wie folgende Beobachtungsnotiz erläutert (Abb. II):

Im Lager werden Schuhe von der Designerin und dem PR-Manager für die Modenschau bearbeitet. Sie bemalen die gebrauchten Schuhe mit Window Colour, denn diese Farbe hat eine silikonartige Textur, die sie mögen.

Abb. 12: Präparierte Schuhe für die Modenschau

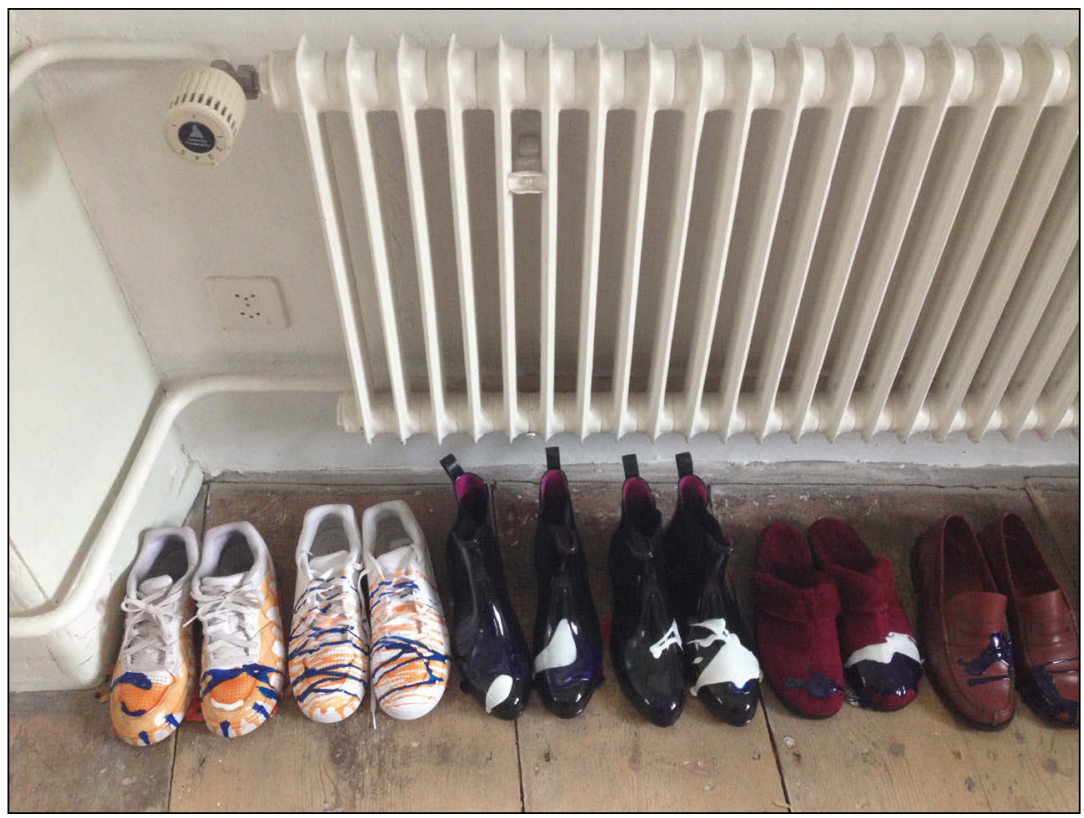

Der Fotograf ist nicht begeistert von den Accessoires (er nennt sie »Pfupf «) und

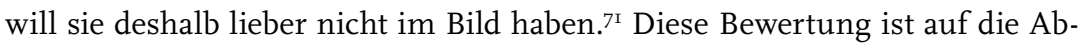
sicht, das Bild möglichst natürlich zu gestalten, zurückzuführen.

71 | Diese Interaktion markiert einen Moment eines Konflikts im Projekt, der aufgrund divergierender Interessen und unterschiedlicher erklärter Ziele entsteht. Wäh- 
Manchmal werden Accessoires nur für den Zweck der Vorführung bzw. der Aufnahme, nicht aber für die serielle Produktion gefertigt. Dann handelt es sich um Show Pieces. Umgekehrt kommt es auch vor, dass Kleider, die für die Modenschau ausgewählt werden, schließlich nicht seriell hergestellt werden, da sie ökonomisch zu wenig lukrativ sind. Trotz solcher ökonomischen Bewertungen sind diese nicht weniger relevant für das Gesamtbild der Kollektion, weshalb deren symbolischer Beitrag zum Gesamteindruck der Kollektion und der kreativen Elemente steigt. Ähnlich wie Key Looks haben Show Pieces einen besonderen Wert, denn sie begünstigen das Marketing und spielen mit dem Wiedererkennungswert der spezifischen Kollektion wie auch dem des Unternehmens. Das Präsentieren dieser Modeobjekte (Kleidung wie Accessoires) ist deshalb wichtig für das Modeunternehmen. Show Pieces sind eine Verschmelzung symbolisch-visueller und materiell-textiler Darstellungen: Als getragener und interpretierter Gegenstand faszinieren sie und ziehen so die Aufmerksamkeit des Publikums auf sich; als exklusive und nicht kommerzielle Objekte dominiert deren Symbolik, die nicht nur dem Objekt, sondern auch der Show einen einmaligen und avantgardistischen Reiz verleiht. So spielen diese Accessoires und Kleider mit den Kennzeichen des Luxus.

Die inszenierenden Shows sind Kommunikationsmittel der Kreativität und spielen somit eine bedeutende Rolle für die Außendarstellung der Modefirmen.

\subsection{Finalisierendes Storytelling}

Als letzte Darstellungsform der Mode ist das Storytelling zu nennen, das die narrativen Fragmente in der Postproduktion zu einem Plot verbindet. Das abschließende Storytelling in Broschüren und Newslettern findet erst nach der Herstellung statt, während Etiketten und Labels eine beständige Form des Storytellings sind. Finalisierend entsteht aus einer Verkettung verworfener und überarbeiteter Versuche eine Version, die als intendierte Geschichte präsentiert wird. Dazu werden Etiketten und Labels, Broschüren und Newsletters entworfen, die als außengerichtete Kommunikation den Höhepunkt der Selbstdarstellung verkörpern. Hinzu kommt, dass die Ausstellungsfläche in den Ladenlokalen szenografisch für die neue Kollektion eingerichtet wird. Das Storytelling verknüpft die orientierenden Narrative (Thema, symbolische und materielle Markierungen) mit denjenigen der Inszenierung (Accessoires, Schauplatz) im finalisierenden Schaubild der Etiketten und Labels, Broschü-

rend die Designerin die Accessoires als Medien des Storytellings braucht, zielt der Fotograf auf "richtige" Bilder ab, von deren Inhalt und Komposition keine fremden Gegenstände ablenken sollen. 
ren und Newsletter und der Szenografie zu einer kohärenten, verständlichen Geschichte. Schließlich wird sie über mediale Kanäle kommuniziert und verbreitet.

\subsubsection{Etiketten und Labels}

Neben den Marketingbemühungen über Newsletter und Broschüren wird direkt am Kleidungsstück auf den Etiketten über den Preis und qualitative Informationen informiert. So sind auf den angenähten Labels die Größe, Produkt- und Unternehmensname zu finden. Etiketten und Labels bleiben über einzelne Kollektionsgeschichten hinweg bestehen. Folglich handelt es sich bei ihnen um beständige narrative Formen, deren Inhalt sich auf das Unternehmen und deren Technologien bezieht.

Erstens sind Etiketten ein Hinweis auf die Beschaffenheit des Kleidungsstücks. Sie machen kenntlich, aus welchem Material das Kleidungsstück besteht und wie dieses bestmöglich gepflegt wird. Etiketten beschreiben die Kleidung und ihre qualitative Beschaffenheit sowie den Umgang damit. Zweitens sind Etiketten ein Hinweis auf die Herstellung des Kleidungsstücks. Das Etikett gibt an, woher die Materialien stammen, wo die Kleidung hergestellt wurde und über welche Stationen der Herstellungsprozess verlief. Das Etikett gibt auch Informationen zur räumlichen Disparität der Herstellung preis. Diese Herstellungshinweise beinhalten Informationen zu Handwerkstechniken, so zum Beispiel »woven on traditional wooden handlooms in Kathmandu«, »hand embroidered in Kolkata«, wozu weiter ausgeführt wird, dass »[w]e were lucky enough to find a young, independent master artisan in Kolkata who embroiders our ideas, philosophy and passion into textile works of art«. Ein anderes Beispiel beschreibt die Verbundenheit mit heimischen Handarbeiten. Auf dem Etikett steht:

Would you have guessed this garment was screen printed in Mitlödi? The tiny Swiss village in the canton of Glarus has an excellent reputation among haute-couture circles for its highly skilled artisans. Mitlödi perfectly embodies our philosophy of injecting traditional local crafts with cutting-edge designs.

Infolgedessen explizieren Etiketten technische Finessen, Produktionsabläufe und geografische Herstellungs- und Zulieferorte. Diese kommunizierte Transparenz kann ein Unterscheidungsmerkmal zu anderen Organisationen, wie auch anderen Sektoren der Creative Industries, sein. Gleichzeitig wird dadurch eine Nähe zur Kundschaft geschaffen: »von uns für dich, hergestellt in der Schweiz« lautet die Kassenbelegaufschrift, die direkt anspricht und eine familiäre, auf Dubasis beruhende Verbindung zwischen Unternehmen und Kundschaft herstellt. Die Ansprache impliziert, dass die Geschichte bei der Trägerin 
weitergeht und nicht durch den Kauf beendet wird. Als Folge wird peinlich genau darauf geachtet, dass jedes Kleidungsstück mit den passenden Etiketten versehen wird. Die Etikettenzusammenstellung wird je nach Produkt individuell und von Hand (meistens vom Verkaufspersonal) angeordnet.

Im Vergleich zu den Etiketten sind die Labels direkt an die Kleidung genäht. Sie sind die Signatur des Unternehmens. Das Label hat einen plakativen Charakter und ist entweder innerhalb des Kleidungsstücks oder sichtbar auf dem Kleidungsstück aufgenäht. Dadurch wird neben den Etiketten auch das Label zum potenziellen Kommunikationsmedium.

Mit der Sichtbarkeit und Platzierung des Labels wird gespielt. Das eine Modeunternehmen näht das Label (ca. I x $3 \mathrm{~cm}$ ) immer sichtbar außen am Saum des Kleidungsstücks auf. Auf dem Label an der Außenseite steht nur der Name des Unternehmens, auf dem an der Innenseite steht zusätzlich »Made in Switzerland«. Die andere Firma spielt mit der Platzierung des Labels. Bei den Schals werden die Labels je nach Modell individuell kreiert, jedoch immer am gleichen Ort platziert und angenäht. Diese ungefähr kreditkartengroße Fläche ist auffällig bunt gestaltet und von Hand gestickt, weshalb sie von der Kundschaft auch schon als kindisch bezeichnet wurde. Auf Kundenkommentare wird üblicherweise eingegangen, und so kann sich eine Kundin das Etikett entfernen lassen, wenn es stört; gleichzeitig wird es als Werbefläche bezeichnet. Wenn die Labels falsch hergestellt wurden - ein Beispiel ist eine weiße Popelinebluse, auf die das weiße Etikett mit weißen Stickereien außen aufgenäht werden soll, die Stickerei ist aber fälschlicherweise rot -, werden die Produzenten darauf hingewiesen und die Fehler behoben. Da die Labels die allerletzte Station der Musterung sind, können Anpassungen bis kurz vor Produktionsstart vorgenommen werden. Etiketten und Labels sind narrative Elemente, die erst abschließend zum Zweck der Außendarstellung (wörtlich!) eingesetzt werden.

\subsubsection{Broschüren und Newsletter}

$\mathrm{Zu}$ den beständigen narrativen Elementen kommen sporadische, also solche, die absichtlich für die kommunikative Darstellung der Kollektionsgeschichte entwickelt werden. Ein Beispiel ist das Narrativ, das aus den unterschiedlichen Posen der Models konstruiert wird. Die Idee der Geschichte wird erst durch die Bildbearbeitung sichtbar. Die Positionen wurden mit Stützen, verschieden hoher Sitzmöglichkeiten und Matten imitiert, um ohne zu fallen eine möglichst natürliche Fallposition herzustellen (Abb. I2). Diese Bildkonstruktion, die in der Postproduktion vom Fotografen oder Grafiker entwickelt wurde, wurde für den Newsletter wie auch die Kollektionsbroschüre verwendet. Der Newsletter wird im Zweiwochenrhythmus verschickt; die Kollektionsbroschüre wird jeweils zur Veröffentlichung der neuen Kollektion elektronisch an die 
registrierten Kunden versandt oder liegt im Laden in physischer Form aus. Für diese Kommunikation werden sowohl die Haptik des Papiers, das Format und die Erscheinungsform (Heft, Poster) bedacht (persönliche Korrespondenz, 03.05.20I7). Die Broschüre umfasst realistische Aufnahmen der getragenen Kleidung, Fokusbilder auf textile Details, aber auch szenische Bilder und Stillleben, Texte, Beschreibungen und Beschriftungen. Auf diese Weise finalisiert das Storytelling die Kollektion und vervollständigt so die kohärent konstruierte Geschichte.

Abb. 13: Beispiel der Postproduktion für den Newsletter

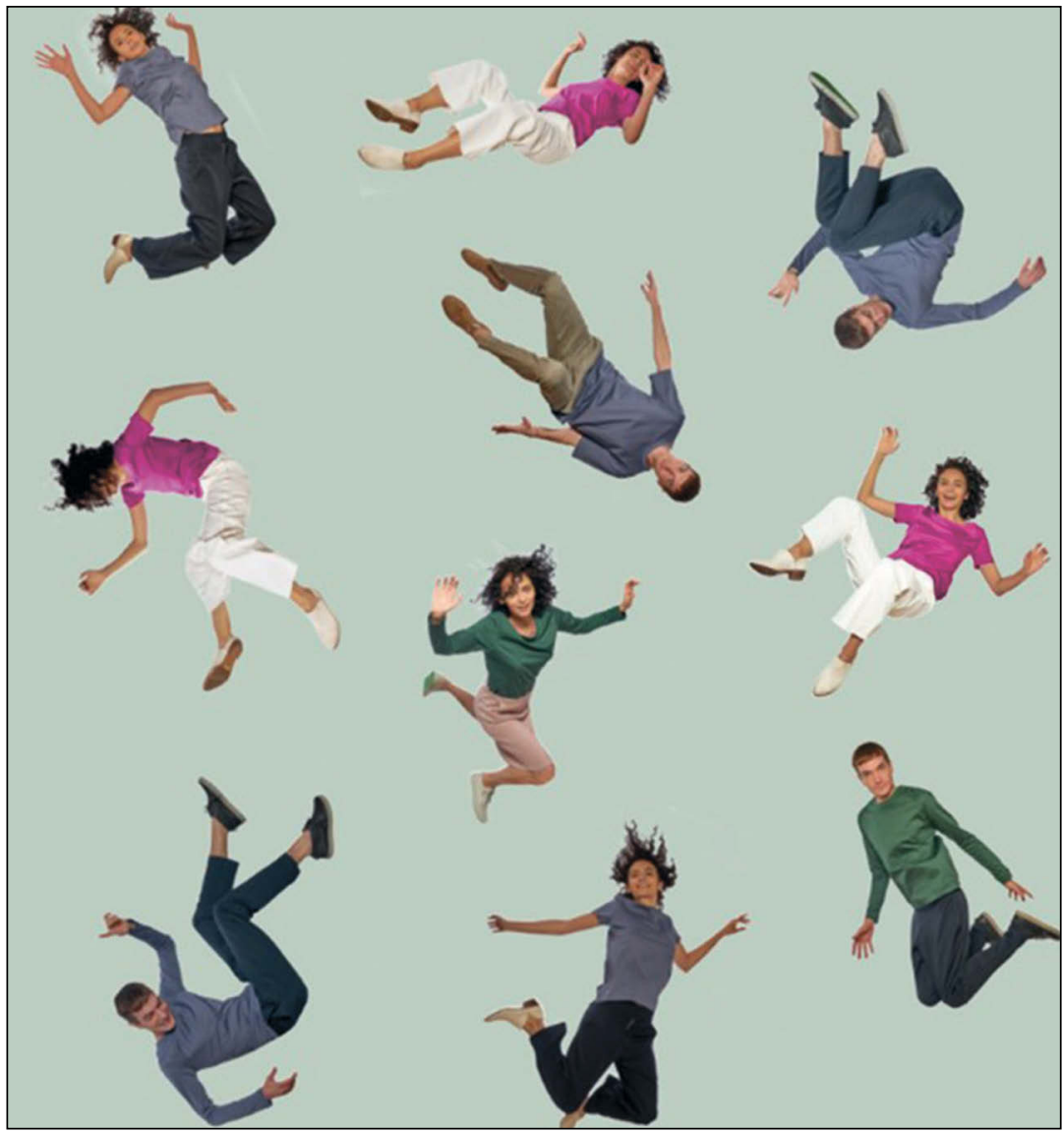

Die Fotoshooting-Aufnahmen, die das Thema der Kollektion aufnehmen sollen, werden nicht selten in der Kollektionsbroschüre mit Texten ergänzt, sodass die visuelle Darstellung der Broschüre den Charakter eines >Handbuchs< oder >Manifests< zur Kollektion erhält. Die Designerin und der Marketingexperte 
stellen eine Broschüre zusammen, die die Geschichte mit Bildern und Texten vervollständigt. Ein Beispiel ist ein südamerikanisches Gedicht, der Verfasser ist ein Familienmitglied, das ergänzend zu den Bildern der argentinischen Kollektion gedichtet wurde und die Geschichte am Ende der Broschüre abrundet (Abb. I3). Weil der Inhalt des Gedichts das Thema der Kollektion aufgreift und in neuer Form erzählt, ist es ein narrativer Bestandteil der finalisierenden Selbstdarstellung. Zudem wird hier auch die spezifische Sprache der Marketingkommunikation offensichtlich. Häufig spiegelt diese Sprache den »künstlerischen« Reichtum des Projektteams aus Designerin, Marketingexperte und zusätzlicher Grafikperson.

Abb. 14: Argentinisches Gedicht aus einer Broschüre

\begin{tabular}{|rl|}
\hline En el ojo el agua vive & Im Loch lebt das Wasser \\
y por el ojo muere el caballo & und wegen des Lochs stirbt das Pferd \\
y el gato y el cuerpo. & und die Katze und der Körper. \\
El ojo de tinta, negro & Das Tinten-Loch, schwarz \\
negra el agua de tanto azul. & schwarz das Wasser vor lauter Blau. \\
Amarilio el ojo en el cielo & Gelb das Loch im Himmel \\
Casi blanco. & Fast weiß. \\
Qué quema mi andar & Wie mein Gehen brennt \\
y mi mano de fuete y laburo & und meine Hand von Peitsche und Arbeit \\
y mi yegua sedienta, & und meine durstige Stute, \\
al ojo me lleva. & bringt mich ans Loch. \\
Viva. & Lebendig. \\
& \\
\hline
\end{tabular}

Die Texte spiegeln die künstlerische Auseinandersetzung mit der Kollektion. Ein illustratives Beispiel ist auch die Sprache, die beispielsweise im angeführten Newsletter gewählt wird:

Die erste Begegnung war die hinreißendste. Ihr Name spricht sich französisch aus: Jupes Volante [sic!]. Sie roch nach exotischer Leichtigkeit und dem ersten Mal Barfußgehen. Lau verfing sich der Wind in der Weite des Saums. Landschaften von 100 Tälern, Flüssen und Schatten bauschten den geschmeidigen Baumwollzwirn auf. Es war Sommer. Ein sommerlicher Konsens und saloppe Kreuzung aus Ferien und Alltag. Jupe Volante [sic!] ist in Scooter, Deep teal und Nachtblau erhältlich und aus $100 \%$ swisscotton gestrickt. 
Die Kollektionsmodelle und -farben werden mit einem poetischen Kommentar in eine imaginäre Szene eingebettet, die ein Bild schafft, das die Kollektion präsentiert und erklärt. Ähnlich dem von Fine (1996) formulierten Argument zu Restaurantküchen und deren Personalsprache, die immer dann aktiviert wird, wenn das Personal einen bestimmten Eindruck kontrollieren will, so wird im vorliegenden Beispiel eine künstlerische Rhetorik gewählt, die einen bestimmten Eindruck vermitteln will. Diese Neuerungsnarrative und kreativen Darstellungen kommen zur Anwendung, wenn Originalität und Neuheit für eine spezifische Arbeitsaufgabe eine besondere Rolle spielen: "The creative display of cooking, the idiosyncratic transformation of foodstuff, and the presentation of food to appreciative, knowledgeable audiences is often labelled art, while routine preparations are not (Fine I996: IOI). Dementsprechend profitiert Storytelling von einer bestimmten $>$ kreativen Sprache.

Organisierende Geschichten markieren den Abschluss einer Kollektion. Im Storytelling werden die bis dahin ziemlich lose gekoppelten narrativen Fragmente nun zu einer abschließenden, zusammenhängenden Erzählung der neuen Kleiderkollektion komplettiert. Dazu dienen narrative Elemente der Finalisierung wie Etiketten und Labels, Kollektionsbooklets und Newsletter, die die antizipierte Geschichte zum Ende visuell illustrieren und textuell ergänzen.

\subsubsection{Szenografie}

Die Szenografie, die Ladengestaltung und die Schaufenstervariationen, sind auch Bestandteil der narrativen und zeremoniellen Schauseite, denn die regelmäßigen Anpassungen und Variationen in den Schaufenstern und die Veränderungen im Ladenlokal weisen darauf hin, dass die Warenausstellung und -präsentation als Bestandteil der kreativen Außendarstellung verstanden wird. Die Bedeutung der Präsentationsweise zeigt sich einerseits in der dafür aufgewendeten Zeit (z. B. in Sitzungen oder der Ladenumgestaltung) als auch in den dafür aufgewendeten Ressourcen. Die Szenografie wird als repräsentative Darstellungsform gewählt mit der Absicht, die Erneuerung im direkten Umfeld kenntlich zu machen.

Für jede neue Kollektion wird das Ladenlokal umgestaltet. Die Gestaltung der Verkaufsfläche variiert mit dem Kollektionsthema. Saisonale Gegenstände, z. B. Saisonschnittblumen oder ein geschmückter Weihnachtsbaum, ergänzen die Kollektion zu einem stimmungsvollen Gesamtbild. Während eine Geschichte eine Kollektion lang erzählt wird, variiert die räumliche Interpretation dieser in kürzeren Abständen. Dazu werden Möbel verschoben, weggeräumt oder hinzugestellt, neue Produkte ausgestellt, alte Produkte neu geordnet und das Licht wöchentlich von einem Elektriker kon- 
trolliert. Für besondere Anlässe werden Handwerker angeheuert, wie z. B. ein befreundeter Schreiner und Produktedesigner, der mit der Weihnachtsschaufenster-Gestaltung beauftragt wird. Die regelmäßigen, szenografischen Änderungen sind ein bedeutungsvolles Element für das abschließende Narrativ und die Außendarstellung.

Auch die schlichte Ausstaffierung des Ladenlokals, die den reinen Stil der Mode aufnimmt, ist eine Möglichkeit der szenografischen Ausgestaltung. Diese Schlichtheit ist ein essenzieller Bestandteil der Kommunikation mit dem Publikum. Da durch das Schaufenster das Licht in den Raum dringt, müssen die Kleider entsprechend farblich richtig geordnet werden. Die Schaufenstergestaltung ist deshalb auch Thema einer Sitzung, in der die neue Kollektion vorgestellt wird und die Designerin den Shop-Verantwortlichen erklärt, wie die Läden und die Fenster zu gestalten sind:

Wichtig ist, dass die hellen Farben vorne im Laden hängen und die dunklen eher in den Hintergrund, also hinten in den Laden geräumt werden. Es betrifft alle Lokale, weil alle "offene" Schaufenster haben und da nicht viel drin ist. Das heißt, dass der Passant/die Passantin rechts und links vom Plakat von den Einrichtungen des Ladens abgelenkt würde und das Schaufenster auch oft eher wild eingerichtet ist. Es sei deshalb wichtig, dass die Modelle akkurat hingelegt und auch die Farben eher ruhig kombiniert würden, denn nur mit Ruhe im Schaufenster schafft man es, die Passanten zu stoppen und ihre Aufmerksamkeit zu gewinnen.

Hierbei steht das Licht-Farben-Konzept im Vordergrund, auf welches das Verkaufspersonal ausführlich hingewiesen wird. Der reduktionistische Ansatz verlangt, dass die Aufmerksamkeit weniger über opulente Schaufenstergestaltungen denn schlichte Arrangements gelenkt wird. Die Szenografie des Ladens und Schaufensters wird als Mittel der Darstellung neuer Kreationen eingesetzt und variiert mit der Erneuerung der Kollektion. Als solches ist sie eine Voraussetzung der Kommunikation von Kreativität. 


\subsection{Kommunikative Aspekte, Narrative und Zeremonien}

Modefirmen konstruieren über ihre Sprache (narrative Fragmente in Storytelling und Szenografie) und Zeremonien (Shows) eine Schauseite, die institutionellen Erwartungen und Rationalitätsmythen entspricht. Modedesign gründet also nicht nur auf sorgfältiger Entwicklung und technologischer Produktion kreativer Güter, sondern beruht auch auf einer kommunikativen Darstellung des Neuen. Während der Entwurfs- und der Vermarktungsphase werden im sprachlich-zeremoniellen Spiel mit narrativen Fragmenten symbolische und materielle Orientierungsmarkierungen konstruiert, die in den Shows inszeniert und im finalisierenden Sprachspiel aufgegriffen werden. Mittels dieser Methoden werden eine authentische Außendarstellung und eine kreative Fassade konstruiert. Das Ziel dieser Darstellung ist es, trotz standardisierter Produktions- und Arbeitskonzepte nicht nur nach außen hin glaubwürdig, authentisch und kreativ zu erscheinen, sondern diese Glaubenssätze auch intern zu verifizieren und zu übernehmen. Diese kommunikativen Aspekte werden als Kreativitätszeremonie und Neuerungsnarrativ bezeichnet, denn im Vergleich zur materiellen Produktion von Kreativität wird hier ein sinnbildhaftes Organisationsporträt gezeichnet, das auf zeremoniellen und sprachlichen Darstellungen beruht. Im Anschluss auch mithilfe des soziologischen Neoinstitutionalismus konnten Sprache, z. B. Vokabulare, und Zeremonien als zentrale Organisationsformen der Darstellungsformen in der Mode analysiert werden. Kreativitätszeremonien und Neuerungsnarrative sind Darstellungsformen der Kreativität und ergeben die kommunikativen Aspekte der Kreativitätshervorbringung.

Auf der Basis des Feldmaterials illustriert eine Darstellung, mit welchen Mitteln und Methoden der >profane< Produktionsprozess »kreativisiert« und das Label Kreativität reklamiert wird (Abb. I4-I7). Dabei wurden folgende Darstellungsformen der Mode als Voraussetzung für die Kommunikation von Kreativität beschrieben: generelle Aspekte der Entwürfe (Narrative [Abb. I4]), generelle Aspekte der Inszenierung (Abb. I5) und die je spezifischen Ausprägungen (Fotoshooting und Modenschau [Abb. I6] und der Postproduktion und des Marketings [Abb. I7]).

Die kreative Leitung übernimmt die Aufgabe, das Thema als sichtbarer Stil in die Kollektion einzubauen. Während die materielle Entwicklung und serielle Produktion auf kreativ-technologischen Verhandlungen basiert, werden zur Kommunikation narrative und zeremonielle Elemente glaubhaft dargestellt. Thema oder Arbeitstitel sind ein Beispiel für narrative Fragmente. Die Geschichte wird thematisch in symbolischen Narrativen wie dem Arbeitstitel oder Modell- und Farbbezeichnungen und in materiellen Narrativen wie dem grafischen Logo oder neu gezeichneten Stoffdrucken kenntlich gemacht. In der Entwurfsphase setzen die Narrative den Rah- 
men für das Kollektionslogo, den Stoffdruck und die kreierten Fertigungsdetails, die nicht selten auch in Zusammenarbeit mit Kooperationspartnern entwickelt werden.

Die thematische Veränderung der Kollektion, der Schnittmodifikationen und der Farben illustriert, inwieweit stark Wettbewerb geprägte Industrien größerem Druck ausgesetzt sind, die Produkte stets anzugleichen und so Marktanpassung zu markieren und zu symbolisieren, um den wirtschaftlichen Vorteil aufrechtzuerhalten, jedoch gleichzeitig das Kerngeschäft sowie den eigenen Stil, die Handschrift oder das künstlerische Ideal, unter diesen gespannten Bedingungen fortzuführen. Die narrativen Fragmente werden in den Shows in Form von Outfits, Accessoires und Schauplatz und im Storytelling in Form von Etiketten und Labels oder Broschüren und Newslettern wieder aufgegriffen, sodass retrospektiv ein ideales Kollektionsnarrativ entsteht. Zum Schluss müssen diese Neuerungsnarrative glaubhaft kommuniziert werden.

Inszenierende Shows sind eine Besonderheit der Modebranche, denn sie visualisieren die Neuerung physisch am Menschen, musikalisch interpretiert und örtlich lokalisiert, zelebrieren den kreativen Ausdruck des Modedesigns und sind daher eine Kunstform an sich. Sie involvieren eine Vielzahl unterschiedlicher, temporärer wie auch permanenter Akteure, die der »kreativisierenden« Darstellung der Idealisierung der Modefirmen dienen. 
Abb. 15: Generelle Aspekte des Entwurfs

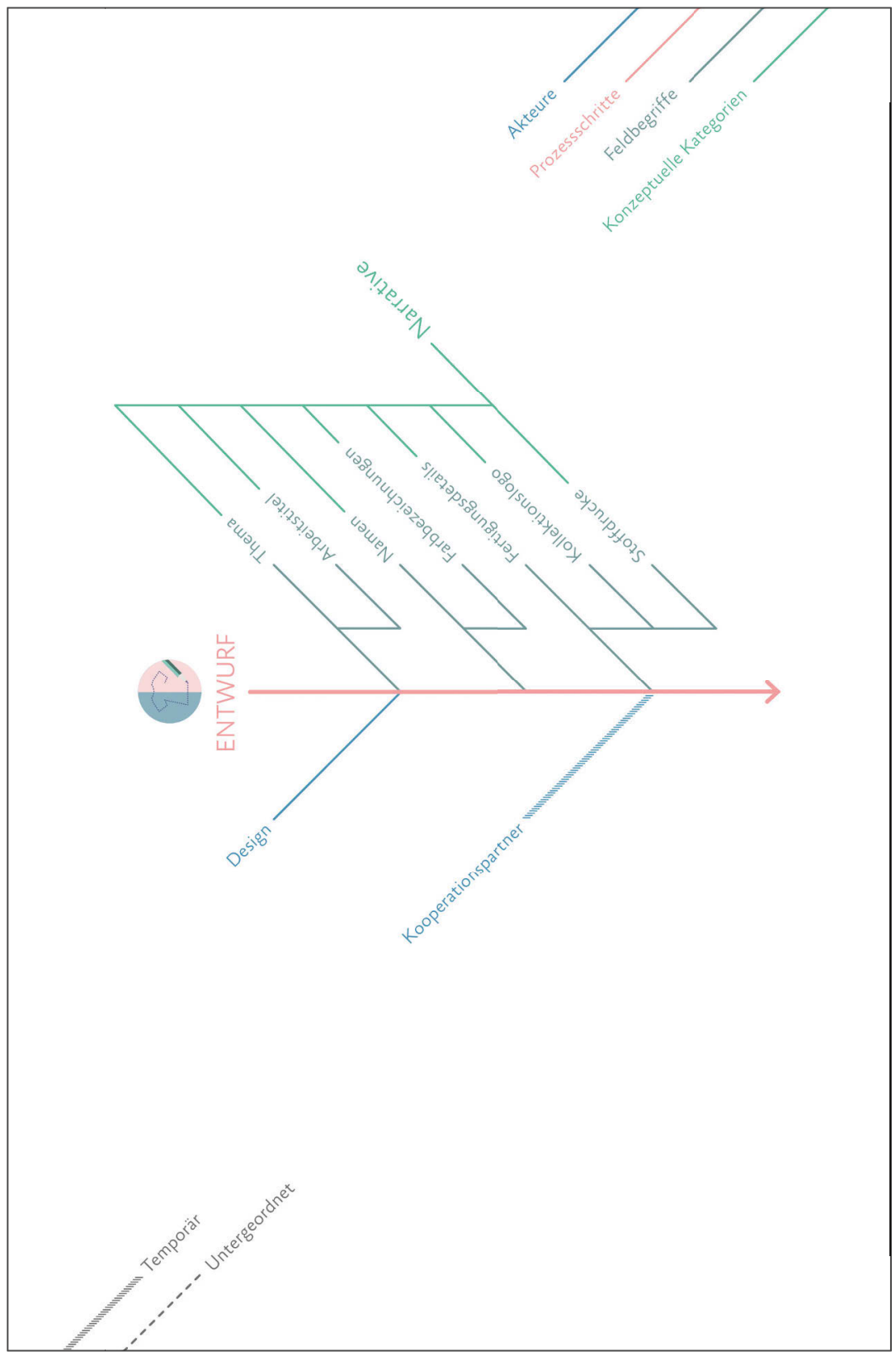


I88 Die Fabrikation von Kreativität

Abb. 16: Generelle Aspekte der Inszenierung (1)

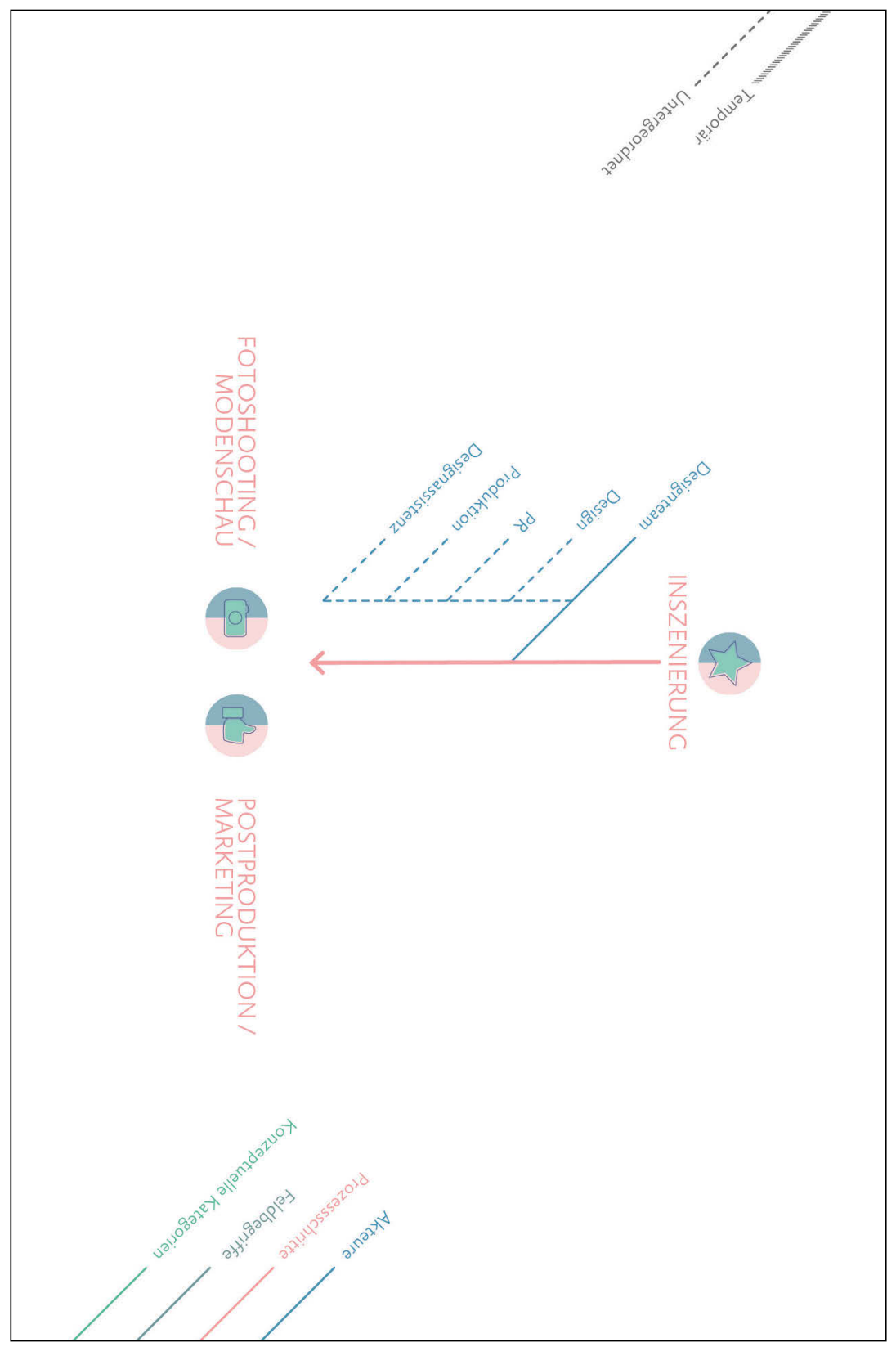


Abb. 17: Spezifische Aspekte des Fotoshootings/der Modenschau (2)

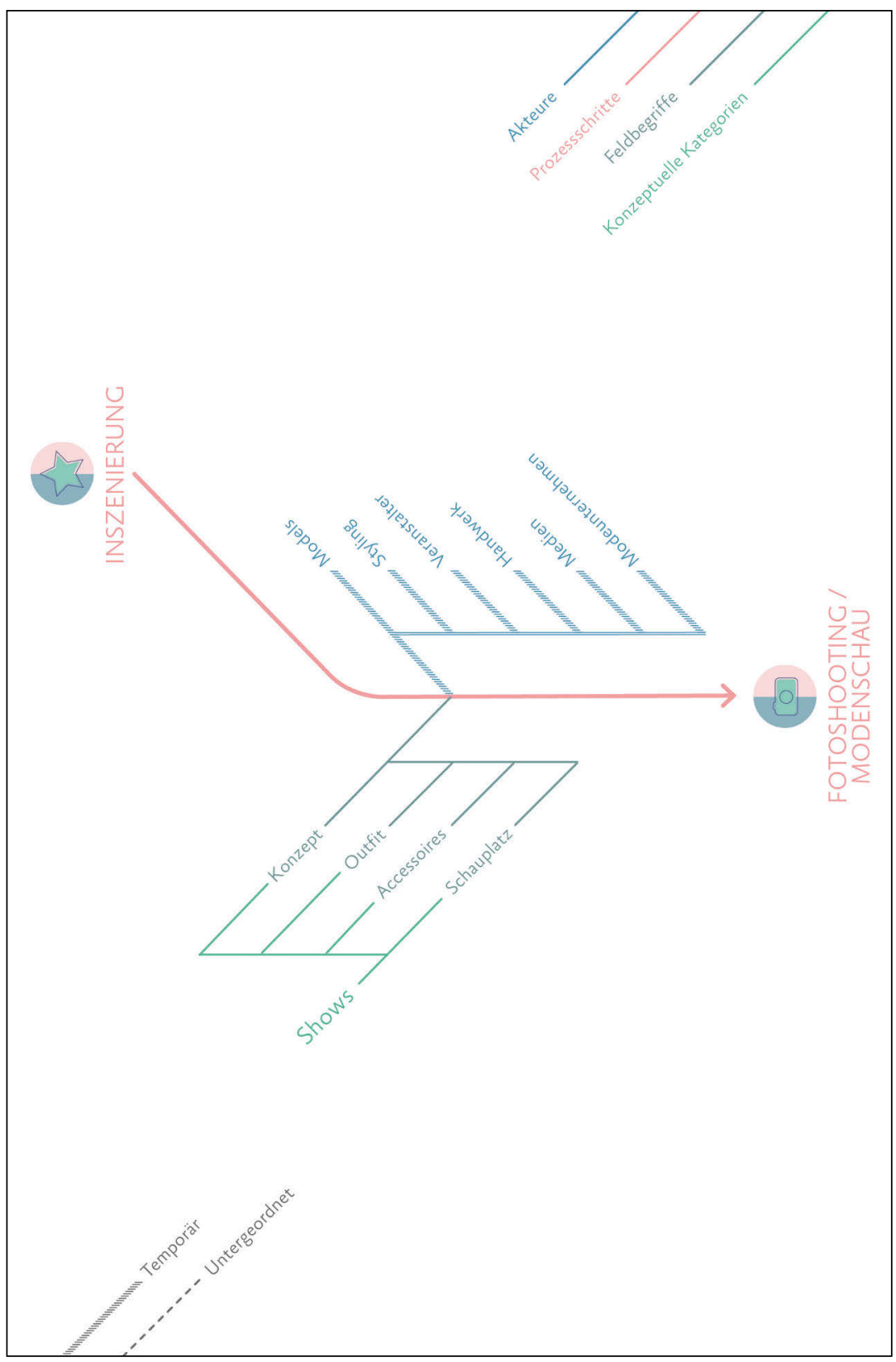


I90 Die Fabrikation von Kreativität

Abb. 18: Spezifische Aspekte der Postproduktion/Marketing (3)

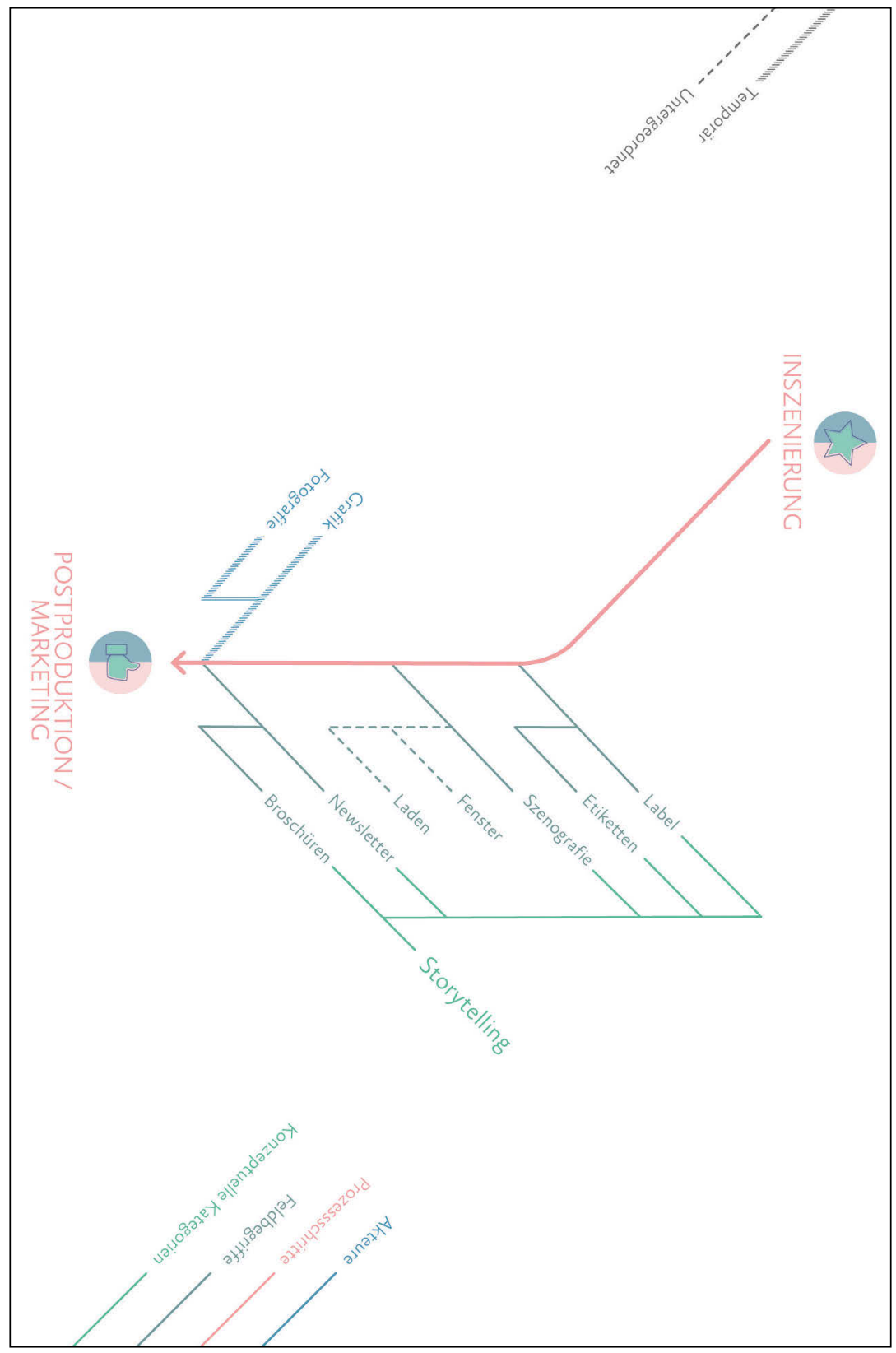


Die orientierenden Narrative werden zur Grundlage für das Konzept der Modenschauen und des Fotoshootings (Abb. I6). Im Konzept werden die grundlegenden Komponenten der visuellen Darstellung beschrieben, die zum Arrangement der Geschichte beitragen. Diese Shows zeichnet aus, dass sie viele Ressourcen (Zeit, Geld, Personal und künstlerische Gestaltung) benötigen und sich nicht nur von anderen Modenschauen bzw. Fotografien abheben, sondern auch von den vergangenen Shows der eignen Firmen unterscheiden.

Abschließend werden narrative Fragmente der Inszenierungen (besondere Accessoires, Show Pieces oder Fotografien, die aus Shootings resultieren) zu einer finalisierenden Geschichte konstruiert. Die Geschichte hat nun keinen fragmentären Ausdruck mehr, sondern vermittelt den Eindruck eines antizipierten Plots. Mit dem Storytelling werden Hinweise in Broschüren und Newslettern verarbeitet und in der Laden- und Schaufenstergestaltung in Szene gesetzt. Dafür werden meist spezialisierte Handwerker (z. B. Schreiner, Grafiker) hinzugezogen. Dieses Zusammenfügen findet ex post in einem Nachbearbeitungsgang statt, der zwar auf die laufende Entstehung und Abwandlung der Narrative Bezug nimmt, diese aber rhetorisch strukturiert und als geplant darstellt. Die genauere Betrachtung der narrativen Fragmente hat jedoch gezeigt, dass die erzählte Geschichte eine emergente ist, die sich sukzessive herausbildet, während die Kollektionsentwicklung rekursiv und nicht geradlinig verläuft.

Die Neuerungsnarrative idealisieren den kreativen Ausdruck und perpetuieren den Mythos der Kreativen und der kreativen Praxis. Anhand von Narrativen wird erklärt, wie Kreative professionell auf gewisse Themen reagieren, sich diese zu eigen machen und künstlerisch umsetzen. Die vorgängig bestimmten Narrative und die zeremonielle Inszenierung der nachträglich konstruierten Geschichte machen Kreativität sichtbar.

Entsprechend unterstreicht das finalisierende Storytelling die institutionelle Passung mit Rationalitätsmythen und Erwartungen aus dem Feld und arbeitet gleichzeitig die individuelle Verschiedenheit $\mathrm{zu}$ anderen Feld- oder Marktteilnehmern heraus: »As such, they [die Erzählenden] selectively distill a complex jumble of otherwise ambiguous and contradictory activities, pronouncements, and impressions into a simplified and relatively coherent portrait « (Ashforth/Humphrey I997: 53). Die Organisation wird auf eine besondere Weise porträtiert, die ihr eine spezifische Marktnische zuweist. Mützel spricht darum von der doppelten Rolle von Geschichten:

Zum einen enthalten Geschichten Informationen und Bewertungen, zum anderen geben sie Auskunft über die strukturelle Position, die der Erzähler für sich und andere ausmacht. So erschaffen Akteure mit Verkettungen von Erzählungen, die evaluierend und strukturierend wirken, gemeinsam den Markt. Der Austausch von Geschichten als 
Netzwerk von Signalen kann so als das Hauptmedium der Marktkonstruktion verstanden werden. (Mützel 2015: 273)

Geschichten sind organisationale Mittel, den Wettbewerb im Feld der Mode zu konstruieren und zu kontrollieren. Insofern sind Erzählungen von Evaluatoren, Kritikern, Journalisten und Regulationsbehörden die evaluative Basis für Zuschreibungen und Bewertungen organisationaler Kreativität. In Anlehnung an die narrative Marktkonstruktion versucht die vorliegende Arbeit zu argumentieren, wie über die Verkettung von Narrativen und Zeremonien Kreativität konstruiert wird.

Alle drei Formen der Kreativitätsdarstellung, orientierende Narrative, inszenierende Shows und Storytelling, dienen der Verankerung von Glaubenssätzen und der Kommunikation und werden als Kreativitätszeremonien respektive Neuerungsnarrative zur Grundlage für Bewertungs- und Deutungspraktiken. Sie »kreativisieren« die wenig spektakuläre Herstellung kreativer Güter und wirken demzufolge als conditio sine qua non der Kreativität im Feld der Mode. Das sprachliche und zeremonielle Ausweisen wird selbst zum Akt der Kreativität: »[T]he attribution of organizational creativity is itself a playful, creative process in which an organization - aiming to be recognized as creative - acts as if this recognition has already been attributed« (Koch u. a. 20I8: 2). Dieses Spiel schließt an die technologischen Aspekte der Kreativität an, indem die unspektakulären Herstellungsweisen kreativer Güter in Szene gesetzt und mit Geschichten illustriert werden, und macht die kommunikativen Voraussetzungen sichtbar.

In diesem Kapitel wurden die sprachlichen und zeremoniellen Darstellungen der Mode als Reaktion auf institutionelle Erwartungen und Kreativitätsmythen analysiert. Anhand dieser Mechanismen wurde versucht zu erklären, wie Kreativität kommunikativ konstruiert wird. Dabei wurde der Frage nachgegangen, welche Rolle Sprache in der Kreativitätshervorbringung spielt. Sprachspiele sind Methoden der organisationalen Selbstkontextierung und Außendarstellung und als solche nicht nur »rhetorische Strategien« (Suddaby/Greenwood 2005) oder »nachträgliche rhetorische Aufbereitung«, mithilfe welcher die Kreativität des Modedesigns im Feld reklamiert wird, sondern vor allem »Praxis alltäglicher Begründungen« (Hasse/Schmidt 2008). Diese sprachlichen Begründungen und zeremoniellen Inszenierungen richten den internen Organisationsfokus (Ziele, Ressourcen, Auftrag) an bestehenden Erwartungen des Feldes aus und stellen - nicht erst im Nachhinein - rentable Verbindungen zwischen Organisation und Umwelt her (Lounsbury/Glynn 200I). Die Kreativitätszeremonien und Neuerungsnarrative, die im Modesektor in Form von kollektionsspezifischen vorgängig entwickelten und nachträglich aufbereiteten Geschichten und showartigen Ereignissen auftreten, aktualisieren vergangene Kreationen und bringen Neuerungen zur Geltung. 


\section{Organisierte Kreativität in der Mode}

Am Ausgangspunkt der vorliegenden Arbeit stand die Annahme, dass Organisationen im Feld der Mode als kreativ wahrgenommen werden. Zudem hat sich während der Feldbesuche herausgestellt, dass es problematisch ist, den Begriff Kreativität zu verwenden, da dieser von den Beobachtungs- und Gesprächspartnern weder kohärent noch konsequent verwendet wurde. Obwohl erste Kontakte und Beobachtungen im Feld zeigten, dass Kreativität kaum Erwähnung findet - und wenn, dann »überschätzt« wird -, spielt sie eine relevante Rolle bei der Entwicklung und Verbreitung modischer Neuerungen. Die Abwesenheit einer geteilten Begrifflichkeit und die Beobachtung der Kreativitätserwartung inspirierten diese Arbeit und wurden zum Ausgangspunkt für das weitere abduktive Vorgehen des Theoretisierens, das sich zwischen empirischen Beobachtungen, theoretischen Konzepten und möglichen Erklärungen hin- und herbewegt. Zunehmend entwickelte sich der Eindruck, dass Kreativität ein Label einer institutionellen und sozial-konstruierten Zuschreibung ist. Vor diesem Hintergrund erschien es sinnvoll, der Frage nachzugehen, wie das entsteht, was als kreativ und modisch reklamiert und von der Umwelt als solches bewertet wird: Wie wird Kreativität hervorgebracht? Wie werden Neuerungen im Feld der Mode entwickelt, designt und schließlich erfolgreich kommuniziert? Welche Organisationsformen und Bedingungen sind dabei zentral und welche Akteure sind daran beteiligt? Die kreative Selbstbeschreibung des Creative Directors und der Creative Industries wurden zum Ausgangspunkt genommen. Schließlich konnten anhand des Feldmaterials zwei Ausprägungen von Kreativität analysiert werden: die technologische und die kommunikative.

Die technologische Entwicklung und Produktion kreativer Güter ist stark von Projekten, Etappenzielen und Sequenzierungen geprägt (Kap. 6). Deshalb wird oftmals auf Routinen, repetitive Verfahren und Zeitlichkeit zurückgegriffen oder die Neuerungen an Vergangenem ausgerichtet bzw. an Stil und Tradition orientiert (Kap. 6.I und 6.2). Zudem hat sich gezeigt, dass im Rahmen der Projekte unterschiedliche Akteure aus verschiedenen Organisationen eingebunden sind (Kap. 6.3). Dabei fällt auf, dass sich die Wiederholung mit Spezialisten auszahlt, während Kooperationen mit künstlerischen Gestaltern selten wiederholt werden und einmalig bleiben. Gleichzeitig, um trotzdem einen 
kreativen Anschein zu erwecken, wird die Kommunikation und Darstellung von Kreativität mittels Narrativen und Inszenierungen entwickelt (Kap. 7). Dadurch akzentuieren und erweitern Modefirmen wünschenswerte Eigenschaften und verschaffen sich durch geringe Verwendung anderer, ungewohnter Merkmale Akzeptanz. Bei diesen narrativen und zeremoniellen Idealisierungen zum Zweck des kreativen Anscheins handelt es sich nicht nur um retrospektive, rhetorische Strategien, sondern auch um schon vorgängig festgelegte oder während der Kreation entwickelte Narrative (Kap. 7.2 und 7.3). Diese Formen generieren prospektiven Sinn und ihnen wird eine große Bedeutung zugeschrieben; die Beteiligten sind davon überzeugt, dass ursprüngliche Überlegungen zur späteren Darstellung der Kreation einen großen Einfluss auf die Weiterentwicklung haben und sich - wenigstens zu einem gewissen Teil - auch gegenüber ökonomischen und technisch-rationalen Kriterien durchsetzen, die den Produktionsprozess dominieren. Durch Narrative und Zeremonien wird der Erfolg der Kommunikation erhöht. Vor diesem Hintergrund hat diese Arbeit den Blick auf die Mode als ein Bereich gerichtet, in dem Neuerungen nicht nur mit hoher Verlässlichkeit hervorgebracht werden, sondern in dem auch überaus rigide, zeitliche Vorgaben einzuhalten sind. Die Analyse der Bedingungen und Fragen der Entwicklung, Produktion und Kommunikation im Modesektor ist einerseits ein Beispiel der Aktivierung bestimmter Kooperationen, andererseits aber ein Beispiel für die normative Kreativitätserwartung, die durch institutionelle Passung und zeremonielle Aktivitäten adressiert wird. Vor diesem Hintergrund verbindet die Analyse der Kreativitätshervorbringung im empirischen Fall der Mode Kooperationen und Projekte mit Legitimitätsfassaden und sprachlicher Verankerung von Glaubenssätzen und thematisiert dementsprechend die soziale Bestimmung technologischer und sprachlicher Hervorbringungs- und Darstellungsformen.

Was sind also spezifische Effekte, die mit diesen Organisationsbedingungen einhergehen? Die Modefirmen verzichten auf radikale Brüche, wodurch Fehlschläge reduziert werden. Die Modeindustrie bringt Kreativität als Resultat schöpferischer Rekombinationen oder Neukreationen hervor, jedoch verbreitet sich nur ein Bruchteil davon als modische Innovation mit Langzeitwirkung. Hierbei wurde argumentiert, dass die Umweltbeobachtung weniger eine handlungsweisende denn eine inspirierende Funktion hat, während die Organisation fortführt, was sie beherrscht. Diese Studie diskutiert den technologischen und den kommunikativen Einfluss auf die Unsicherheitsreduktion der Bedingungen der Hervorbringung und Darstellung von Kreativität im Modesystem bzw. in den Creative Industries. Ein weiterer Effekt hat die Temporalität, denn es besteht nur wenig Zeit für die anschließende Diffusion der Neuerung. Radikale Innovationen der Mode sind selten, da Mode auf Zeit angelegt ist und aufgrund des schnellen Reproduktionszyklus oft kaum dif- 
fundieren können. Insgesamt lassen sich deshalb zumeist inkrementelle und weniger radikale Variationen beobachten.

Infolgedessen wurden theoretische Konzepte des Kontingenzansatzes und des Neoinstitutionalismus gewählt, um die empirischen Befunde zu erläutern. Die Forschungsperspektive und die Theoriekonzepte der Kontingenzforschung sowie deren Weiterentwicklungen im Neoinstitutionalismus liefern ein tragfähiges Fundament für die Kreativitätsforschung. Im Dialog befähigen diese beiden Organisationstheorien dieses Forschungsvorhaben dazu, alternative Erklärungsmodelle für die organisationstheoretische Fundierung der soziologischen Kreativitätsforschung zu entwickeln. Die Analyse des Feldmaterials untermauert das theoretische Argument.

Mithilfe des Feldmaterials wurde versucht zu zeigen, dass die Hervorbringung organisationaler Kreativität im Feld der Mode und der damit verbundenen Erneuerungs- und Kreativitätsmythen weder ausschließlich mit der kontingenztheoretischen Annahme der Abschottung zentraler Technologien im Betriebskern noch mit dem neoinstitutionalistischen Argument legitimierter Sprache erklärbar ist. Als Folge unterschiedlicher Rationalitäten, die in den Creative Industries vorherrschen, kann weder nur die Entwicklung und Produktion kreativer Güter mittels technologischer Kontinutität und Effizienz noch die kommunikative und zeremonielle Ausgestaltung kreativer Tätigkeiten die Kreativität einer Organisation reklamieren. Die Verzahnung der beiden Theorien löst die Spannung der losen Kopplung der technologischen Aktivitäten im Betriebskern und der zeremoniellen Inszenierung als Reaktion auf institutionelle Erwartungen der Umwelt. Zwischen sichtbaren Formalstrukturen (Routinen, Technologien, Qualifikationsanforderungen und Kompetenzen) und Aktivitätsstrukturen (Narrative, Inszenierungen und Storytelling) besteht ein interdependentes Verhältnis. Während Modeorganisationen der Logik der Neuheitsroutine folgen und auf der Basis von Routinen kreative Güter produzieren, wird die Neuerung nach außen durch Zeremonien inszeniert und kreativ dargestellt. Bei Ersterem ist hier von technologischen Aspekten der Kreativität die Rede, beim Zweiten wird dies als kommunikative Aspekte der Kreativität bezeichnet. Damit wird die »romantische« Vorstellung von Kreativität (d. h. der Geniemythos wie auch die Einzigartigkeit der Creative Industries) infrage gestellt und mit einer organisatorischen Vorstellung derselben ersetzt.

Diese Arbeit versucht die Entwicklung von Kreativität mittels Standardisierung, d. h. Routinen und Wiederholung, zu theoretisieren. Am Beispiel der Mode problematisiert diese Arbeit die Ambivalenz zwischen Technologie und Sprache, Entwicklung und Darstellung, Idee und Intention und stellt dies nicht nur als komplexen Sachverhalt, sondern ebenso als iteratives Spiel mit festgelegten Gestaltungsgrenzen dar. Der Ertrag der Arbeit trifft folglich nicht nur auf die kreative Tätigkeit der Modeindustrie zu. Die Konzepte Neuheitsroutine und Kreativitätszeremonien bzw. Neuerungsnarrative zeigen die komplexe 
Verschachtelung der Rationalitäten, die in Organisationen der Creative Industries vorherrschen. Die Theoretisierung des Konzepts der Kreativität in zwei konstitutive Dimensionen und die Beschreibung der entsprechenden grundlegenden Eigenschaften können deshalb nicht nur auf »kreative« Industrien oder Organisationen, sondern allgemein auf die Hervorbringung von Neuheit angewendet werden.

Das Kapitel fasst rückblickend den konzeptuellen Argumentationsgang zusammen (Kap. 8.I), skizziert den gewinnbringenden Erkenntnisertrag für die Kreativitätsforschung (Kap. 8.2) und eröffnet einen Ausblick in weiterführende Forschungsfragen (Kap. 8.3).

\subsection{Das Prinzip des Kreierens neuer Moden}

Im Rahmen dieser Arbeit wurde Kreativität als kollektiver, organisatorischer und institutioneller Sachverhalt rekonstruiert. Die kollektive Perspektive betont die Zeitdimension des Kreierens, die aufgrund der spezialisierten und vielzähligen Akteure beschleunigt wird. Die organisationale Perspektive betont die organisatorische Koordination aller beteiligten Akteure, die Verwaltung betrieblicher Formal- und Aktivitätsstrukturen und das Organisieren der unspektakulären Routinen. Die institutionalistische Perspektive adressiert die Rationalitätsmythen im Feld der Mode und die Definition, die Kreativität als Selbstzweck fern von Rechenschaftspflicht verortet. Insofern stellt die sprachliche Konstruktion einen zentralen Baustein der Hervorbringung von Kreativität dar, die sowohl dem zeremoniellen Ausweisen als auch dem glaubhaften Verfestigen des Kreativitätsmythos zugrunde liegt. Das heißt, dass modisch ist, was im Feld der Mode produziert wird, und kreativ ist, was Kreative (er-)schaffen. Daraus gehen zeremonielle Ausgestaltungen, z. B. der Selbstinszenierung, und die Konstruktion von Fassaden hervor, die der Aufrechterhaltung des kreativen Porträts dienen und Glaubwürdigkeit und Vertrauen reflektieren. Modenschauen, Fotostrecken oder die Starallüren der »kreativen Köpfe« sind Beispiele solcher Zeremonien.

Aus der Literaturübersicht geht hervor, dass die Herstellung neuer Kreationen in Produktions- und Kooperationsnetzwerken stattfindet, in denen die Modefirmen eingebettet sind und durch welche Kreativität als Kollektivgut entsteht. So wurden die Creative Industries als Konglomerat von Industriezweigen und Branchen beschrieben, welche Beziehungen, Informationen und Ressourcen über das Produktions- und Kooperationsnetzwerk verwalten und kontrollieren. Netzkontakten kommt eine eminente Rolle in der Herstellung von Modekollektionen zu. Ebenso ist die umfassende Vernetzung im institutionellen Feld lebenswichtig für die symbolischen Ausprägungen der Kreativität (beispielsweise in Zuständigkeiten und Verantwortlichkeiten bestimmter 
Berufskategorien oder Aufgabengebiete), denn darüber werden Ressourcen koordiniert (Informationen, Personal, Trends und Ideen, Publikum) und Legitimität, Beständigkeit in der von Dynamik und Wandel geprägten Umwelt und somit organisationales Überleben generiert. Netzkontakte mit Feldmitgliedern werden im Rahmen der Projektarbeit als Talent- und Informationspool relevant. Sie werden jedoch nur während der terminierten Kooperation aktiviert und deaktivieren sich nach diesen Geschäften wieder bzw. bestehen latent im Mehrzweckressourcenpool weiter. Für das Forschungsvorhaben zur Kreativitätshervorbringung und ständigen und standardisierten Erneuerung der Mode interessieren deshalb insbesondere die permanenten Projektkontakte, die Teil der formalen Organisation sind, sowie die temporär aktivierten Netzkontakte, die zu spezifischen Kooperationsaufgaben angeheuert werden. Ein zukünftiger Forschungsbeitrag könnte Netzwerke deshalb als zwischenorganisationale Räume untersuchen, welche in der Innovationsforschung bereits als »locus of innovation« herausgearbeitet wurden (Powell u. a. I996). Anschließend drängt sich die Frage auf, welche Rolle diese Netzwerke in der Kreativitätshervorbringung übernehmen, die über die Produktion und Koordination kreativer Tätigkeiten hinausgeht. Ein Fokus auf die Netzwerk- bzw. Feldebene wäre eine weitere Weiterentwicklungsmöglichkeit dieser Arbeit und würde zu Fragen der »Konsistenz innerhalb der Divergenz«, d. h. der Heterogenität in Feldern, beitragen (Schneiberg/Clemens 2006).

Die Nachfrageseite kommt im Entwicklungs- und Produktionsprozess modischer Neuerungen als Informationsquelle nicht infrage, da sie erst nach der Fertigstellung der Produkte relevant wird und somit kaum zur Unsicherheitsreduktion beiträgt. Da auch in der Mode das erklärte Ziel ist, »to design items that will please customers « (Aspers/Godart 20I3: I8I), vermeiden Modefirmen totale Neuheit und minimieren mögliche Unsicherheitsfaktoren, indem sie bestätigte Maßstäbe und Modelle abrufen und dadurch an Bekanntes anschließen (sogenannte innovation savers). Die organisationale Perspektive meint deshalb die organisatorischen Ambivalenzen zwischen künstlerisch gestalteten Produkten und zuverlässigen Dienstleistungen, die Ausrichtung auf Erneuerung trotz Bewahrung der Tradition sowie die Spannung zwischen Kommerz und Kulturproduktion.

Modekreationen werden oft in Anschluss an Bestehendes oder Vergangenes entwickelt und entstehen selten »from scratch « (Djelic/Ainamo 2005: 75). Radikale Neuheit entsteht jedoch weniger planbar und mehr zufällig, weshalb Modeunternehmen die Entwicklung mittels bewährter und verlässlicher Verfahren bevorzugen. Die Praxis des Modeschaffens hat aber auch gezeigt, dass diese Verfahren Kreativität nicht hemmen. So werden denn auch Neuentwicklungen selten ohne vorliegende Prototypen und angepasst an vorhandene Organisationsstrukturen und -technologien ausgearbeitet. Meist dient ein fremdes oder ein eigenes Kleidungsstück als Grundlage, das dann abgeändert und 
angepasst wird. Hier findet das Gegenteil einer verfremdenden Gestaltung statt: Der Neuheitsgehalt wird reduziert, um Verständnis zu generieren und die Diffusion respektive die Adoption im Feld zu begünstigen. Aus einer organisationalen Perspektive lässt sich argumentieren, dass Kreativität wesentlich durch die Aspekte Zeit und Wissen koordiniert wird, die gegebenenfalls auch die Institutionalisierung von Kreationen fördern.

Die neoinstitutionalistische Perspektive auf das Phänomen ergänzt die kollektive und organisationale Ausprägung der Kreativitätshervorbringung und leistet eine Erklärung der inszenierten Darstellungen und zeremoniellen Aktivitäten im Feld der Mode. Damit ist gemeint, dass Kreativität weder ausschließlich technologisch koordinier- und planbar noch strategisch so zu verwalten ist, dass ihr die Anerkennung und Zuschreibung aus der Umwelt gewiss ist. Vielmehr zeigt diese Studie, wie das kommunikative Format der Kreativität die technologische Entwicklung der Mode mit sprachlichen und inszenierenden Darstellungsformen ergänzt. Auf der Basis einer erwarteten Zuschreibung wird eine - selber als kreativ wahrgenommene - Inszenierung vorgenommen. ${ }^{72}$ Durch Verbindungen zum organisationalen Feld werden institutionelle Erwartungen bestätigt, Mythen stabilisiert und eine Zuschreibung des Labels »kreativ« überhaupt erst plausibel. Die attributive Verwendung kommt anderen Zuschreibungen, wie z. B. Vertrauen, sehr nahe: Je weniger darüber gesprochen wird, desto glaubwürdiger ist das Label. Die beobachtete Kreativitätsverneinung, der Verweis auf andere Akteure und die Abwesenheit der Begrifflichkeiten im Alltagsgeschäft haben die Annahme bestätigt, dass Kreativität vor allem dann zugeschrieben wird, wenn kaum darüber gesprochen wird. Von einem neoinstitutionalistischen Standpunkt aus kann argumentiert werden, dass Kreativität die Beachtung und Reaktion auf Aktualitäten ist, die aus dem organisationalen Feld stammen und von den Unternehmen beobachtet bzw. übernommen werden (können). Damit ist hier kein gerichteter Prozess gemeint, sondern vielmehr die beliebige, heterarchische und zufällige Verbreitung von (gestalterischen) Elementen, die den Kollektionen einflussreicher und bedeutender Modefirmen oder bekannter Designer entstammen, deren Innovationen in Modemagazinen oder in den digitalen Medien abgebildet

72 | Zu Beginn stand die Annahme, dass Kreativität implizit und nicht über einen aktiven Prozess zugeschrieben wird. Ein Beispiel für eine aktive Zuschreibung sind die Oscars im Filmsektor oder die Billboard Music Charts der Musikindustrie. Jedoch gibt es auch im Modesektor zunehmend Awards, die national vergeben werden, so beispielsweise der Desig Preis Schweiz (Zugriff am 28. Mai 2019 auf www.designpreis. ch/? pageID=482\&Ing=de) oder der Swiss Grand Award for Design, bei dem eine Teilnahme aus der Sparte Mode- und Textildesign auch möglich ist (Zugriff am 28. Mai 2019 auf https://swissdesignawardsblog.ch/who). 
und verbreitet werden. Dabei wäre interessant, weiter zu untersuchen, inwiefern die Unternehmen die Umwelt beobachten und kognitiv Trends anpassen.

Vor diesem Hintergrund wird hier argumentiert, dass Neukreationen erstens mittels Technologien, Routinen und Projekten organisational entwickelt werden, zweitens über Sprache kommuniziert wird, auf deren Grundlage eine Zuschreibung und Bewertung geschehen kann, und viertens Kreationen zum Schluss von Produktionsnetzwerken kollektiv produziert werden. Kreativität in der Mode ist damit sowohl Gegenstand guter Planung mittels Technologien (technologische Aspekte der Kreativität) als auch institutioneller Anpassung durch zeremonielle Ausgestaltungen (kommunikative Aspekte der Kreativität). Folglich hat diese Arbeit ein besonderes Interesse an der Produktion, der Kommunikation und Darstellung sowie der Zuschreibung von Kreativität.

\subsection{Lose Kopplung von Technologie und Sprache}

Die Analyse des empirischen Feldmaterials hat gezeigt, dass Kreativität in Modefirmen erstens auf Routinen basiert, durch Technologien hergestellt und in Projekten ausgearbeitet wird und zweitens mittels Sprache vermittelt und in kreativen Inszenierungen zeremoniell sichtbar gemacht wird. Die Diskussion der technologischen Bedingungen und Fragen der Produktion und der kommunikativen Voraussetzungen und Fragen der Darstellung fließen in die Kreativitätshervorbringung durch die Modefirmen ein. Obwohl es sich dabei um »zwei Seiten derselben Medaille« handelt, bedürfen diese Prozesse einer bestimmten Ordnung. Die Kerntechnologien, im spezifischen Fall das Expertenwissen der Produzenten und das Technikkönnen der Strickcomputer, folgen einer rationalen Logik, während die kreativen Tätigkeiten einer künstlerisch-kreativen Logik folgen. Interessanterweise gehen die kreativen Tätigkeiten über das Entwerfen und Kreieren neuer Moden hinaus und umfassen das stete Beobachten der modischen Umwelt, das Adoptieren daraus hervorgehender Trends und die Übersetzung dieser in die organisationale Technologie. Expressive und symbolisch geladene Güter entstehen folglich nicht (nur) durch expressive Techniken. Die rationale und die künstlerisch-kreative Logik konfligieren jedoch, weil Erstere auf dem ökonomischen Effizienzstreben, Letztere auf der bedeutsamen Neuheitssuche beruht. Die Lösung in Konflikt stehender Erwartungen ist die Entkopplung der zentralen Kernaktivitäten und der Erneuerung der Mode. Als Folge werden die beiden Technologien hermeneutisch vor Störungen und Variation abgeschottet und gleichzeitig wird versucht, Variation und Wandel zuzulassen. Modefirmen müssen einen Weg zwischen der Produktionssicherheit und operativen Kontinuität der zentralen Herstellungsverfahren und der Varianz und Veränderung durch neue Moden finden. Die technologischen Aspekte der Kreativität beziehen sich deshalb auf 
den Übersetzungsprozess, wodurch sich Modefirmen Erwartungen und Ideen aus der Umwelt aneignen. Andererseits, so das Argument dieser Arbeit, generieren Modefirmen im Zuge dieser technologischen Übersetzung sowohl institutionelle Passung als auch Differenzierung innerhalb ihres Feldes. Die kommunikativen Aspekte beziehen sich auf die Ausgestaltung der stilistischen Ausdifferenzierung, wodurch sich Modefirmen von anderen - auch eigenen ehemaligen - Kollektionen abheben. Dieser Prozess resultiert in der kognitiven Nischenbildung und Kategorisierung. Die Kombination von technologischen und kommunikativen Kreativitätsaspekten lässt stilistische Variation zu und ermöglicht trotz institutioneller Ordnung Heterogenität im Feld der Mode. Diese Studie zeigt, wie Modefirmen Stabilität und Wandel sowie Doppelung und Erneuerung bewältigen.

\subsubsection{Lose Kopplung in der Modekreation}

Das Prinzip der losen Kopplung ermöglicht das Puffern des Betriebskerns vor Umwelteinflüssen zugunsten der Kreativitätshervorbringung. Deshalb wird mithilfe der Kontingenztheorie diese als organisatorische Lösung für das gesellschaftliche und organisationstheoretische Dilemma der Inkompatibilität von Rationalität und Unbestimmtheit identifiziert. Die lose Kopplung von Aktivitäts- und Formalstruktur hebt die Dialektik zwischen »lose« und »gekoppelt« hervor. Organisationen stehen der komplexen Situation gegenüber, gesellschaftlichen Vorstellungen nachzukommen und Konformität mit ihnen zu symbolisieren, während die technologische Wertschöpfung im Kern fortgeführt wird. »Thus, the concept of loose coupling allows theorists to posit that any system, in any organizational location, can act on both a technical level, which is closed to outside forces (coupling produces stability), and an institutional level, which is open to outside forces (looseness produces flexibility)« (Orton/Weick I990: 205). Auf der technischen Ebene finden Entwicklungsproduktionsprozesse statt, die zur Stabilität beitragen, während in der institutionellen Ebene Darstellungsformen angesiedelt sind, wodurch Flexibilität und Erneuerung zugelassen werden. Berührungspunkte bleiben also bestehen und sind relevant, denn die Technologie hat großen Einfluss auf die technische Umsetzung der kreativen Idee, so, wie die kreative Idee existierende Technologien herausfordern kann. Die lose Kopplung erklärt sowohl die Agiliät und Anpassungsfähigkeit der Designentwicklung als auch die schnellen Produktionsprozesse.

Durch die Dialektik von technologischen Operationen und institutioneller Anpassung zeigen Organisationen sowohl Anpassungsfähigkeit als auch Eigenarten gegenüber ihrer Umwelt. Auf der Basis der Mikroprozesse der täglichen Handlungen wird ermöglicht, die standardisierten, legitimierenden und formalen Strukturen trotz verkomplizierenden, institutionellen Mythen 
aufrechtzuerhalten. Folglich können sich Organisationen ähnlicher institutioneller Herkunft formal gleichen, unterscheiden sich jedoch stark in den praktischen Ausführungen. Diese Variabilität der Reaktionen auf institutionellen Druck ist auf die Mikroprozesse der Institutionalisierung zurückzuführen, d. h. auf die praktische Umsetzung der Organisationsmitglieder, die darum bemüht sind, sowohl den externen Ansprüchen als auch internen Forderungen gerecht zu werden, weshalb sie Skripts verhandeln, adaptieren und in Alltagshandlungen übersetzen. Mode ist ein Beispiel für ein System, das nur partiell gepuffert ist. Eine detaillierte Untersuchung der Mode trägt deshalb zu einem gleichzeitig distinktiven wie responsiven, von Orton und Weick (I990) als dialektisches Verständnis loser Kopplung bezeichnet bei. Weil das institutionelle Umfeld von Reformen und unterschiedlichen Nachfragen durchsetzt ist, tendieren Organisationen dazu, sichtbare Strukturen und Prozesse, nicht aber ihre Arbeitsaktivitäten anzupassen: »As a consequence, change is predominantly related to formal and visible structures and processes. [...] In order to decouple, facades need to be set up and maintained not only at the interfaces with the external environment, but also internally« (Hasse/Krücken 2008: 200 f.). Lose Kopplung, so wird im Neoinstitutionalismus postuliert, bezieht sich auf Organisationen und deren Notwendigkeit, die Strukturen und Prozesse in Reaktion auf die externen Bedürfnisse anzupassen.

Die Kreativitätszeremonien und die Neuerungsnarrative sind das symbolische Resultat der losen Kopplung zwischen Formal- und Aktivitätsstruktur, wozu die kommunikativen Aspekte in Form von spezifischen, institutionalisierten Vokabularen und gestalteten Fassaden den Glauben an Kreativität konstruieren und die damit verbundenen Mythen perpetuieren. Je weiter die Handlungen und Entscheidungen der Organisationen von dem Bild entfernt sind, das die institutionelle Umwelt davon hat, desto wichtiger werden Kreativitätszeremonien und Neuerungsnarrative, die kreative Fassaden errichten und aktualisieren. Je >profaner die Hervorbringung und je mystifizierter die institutionelle Vorstellung davon, desto größer die Kluft zwischen den technologischen und kommunikativen Voraussetzungen - und umso relevanter die zeremonielle »Kreativisierung « der »demystifizierten« Realität. »Kreative« Organisationen betreiben deshalb vergleichsweise viel Aufwand für die Errichtung kreativer Fassaden, auch wenn man allgemein vermuten kann, dass sämtliche Organisationen zunehmend mehr in ein derartiges Fassadenmanagement investieren. Solche Fassaden sind z. B. die Berufsbezeichnung »Creative Director«, die Einrichtung eines Ateliers anstelle eines Büros oder die Erfindung eines inhaltlichen Kollektionsthemas. Damit wird die Glaubwürdigkeit, dass es sich hierbei um eine kreative Organisation handelt, erhöht und das zugeschriebene Label »kreativ« ratifiziert. Sprache ist somit kein Zufalls- oder Nebenprodukt des Organisierens von Mode, sondern die entscheidende Grundlage, die die kreative Fassade pflegt. Durch Sprache und Zeremonien werden die institu- 
tionellen Erneuerungserwartungen erfüllt und der Glaube an die Richtigkeit des Kreativitätsmythos - »die«, d. h. die Organisation bzw. die Designer, »sind wirklich kreativ« - erhalten. Der Kreativitätsmythos ist somit eine unhinterfragte Rationalität und ist wesentlicher Bestandteil des Modesektors.

Die Modefirmen reklamieren Kreativität, indem sie diese sprofanen< Herstellungsweisen und die daraus resultierenden Güter mittels Storys, Shows und Storytelling als Neuerungen darstellen und dadurch »kreativisieren«. Damit wird das Unspektakuläre spektakulär gemacht; was vorher unsichtbar bzw. effizient war, wird nun sichtbar bzw. aufwendig inszeniert und zu einer Darstellung der Kreativität. Sowohl die technologischen wie auch die kommunikativen Aspekte sind Voraussetzung der erfolgreichen Entwicklung und Produktion, Kommunikation und Darstellung und Zuschreibung von Kreativität im Feld der Mode.

\subsubsection{Technologische Bedingungen und Fragen der Produktion}

Die Beobachtung der Hervorbringung organisationaler Kreativität im Feld der Mode gibt Aufschluss über drei für die organisierte Hervorbringung von Kreativität fundamentale Organisationsformen: Routinen, Technologien und Projekte. Diese Organisationsformen sind das Ergebnis der Konfiguration dreier unterschiedlicher Spannungsverhältnisse. Routinen sind einerseits variationsfähig, andererseits verlässlich, weshalb sie in Organisationen in Form standardisierter Handlungsprotokolle vorkommen oder als Leitlinien situative Interpretationen zulassen. Ähnlich verhält es sich mit Technologien, die, wenn sie gemäß Schema operieren, Beständigkeit versprechen, aber wenn sie partiell modifiziert oder mit Neuem kombiniert werden, auch mal Unterbrechungen auslösen. Schließlich bilden Projekte, d. h. projektbasierte Formen des Organisierens, die Schnittfläche zwischen temporär-variablen und permanent-repetitiven Organisationsstrukturen ab. Während die meisten Projekte auf eine Weise in permanente Verwaltungs- oder Koordinationsstrukturen eingebunden sind, ermöglichen sie Veränderung und Erneuerung. Die drei Organisationsformen bilden eine modulare Aktivitätsstruktur, die sich auf die Entscheidungsfindung im Organisationskern auswirkt und die Schnelligkeit und Flexibilität der Ausarbeitung und Herstellungsverfahren der Modebranche erklären kann. Organisationen versuchen, die Unsicherheit und Ambivalenz der Umwelt zu reduzieren, indem sie sich an Technologien halten, die sie beherrschen und die sich bewährt haben. Ein minimales Maß an Variation der Komponenten (Technologien, Routinen und Projekte) wird gestattet. Infolgedessen können technische Verfahren angepasst, Routinen verändert und Projektpartner gewechselt werden. Darunter versteht diese Arbeit die technologischen Aspekte von Kreativität. 
Die Organisationsformen lassen sich einzeln verwalten und anpassen. Das heißt, dass Routinen, Technologien und Projekte als Bestandteile des zentralen Betriebsapparates zwar interdependent sind, als ambivalente Konzepte jedoch individuell und situativ gedeutet werden und somit rasch auf Veränderung reagieren können. Diese modulare Organisationsstruktur ist die Basis für die paradoxe Gleichzeitigkeit der technologischen Abschottungsleistung und der kreativen Anpassungsfähigkeit bzw. für die lose Kopplung zwischen Organisation und Umwelt. In dieser Arbeit wurde die von Modefirmen entwickelte Methode der verlässlichen Generierung von Neuerungen als Neuheitsroutine herausgearbeitet. Sie bewegt sich zwischen Verlässlichkeit und Variation, zwischen Neuerung und Wiederholung sowie zwischen Permanenz und Zeithaftigkeit mit dem Effekt, dass durch routineartige Prozesse Bekanntes bestehen darf, wie auch Diversität - und dadurch Neuheit - entstehen kann. So schließen sich Kreativität und Routine bzw. Wiederholung nicht aus, sondern ergänzen sich vielmehr zu einem Konzeptpaar, das sowohl einen konzeptuellen Beitrag zum Termingeschäft als auch zu den Techniken der Entwicklung und Produktion von Moden leistet. Ebenso soll der Fall der Mode veranschaulichen, dass Struktur keineswegs etwas ist, das Organisationen haben (oder blind übernehmen), sondern etwas, das sie (aktiv) schaffen.

Die Kontingenzforschung beleuchtet die Technologiezentriertheit innovativer Organisationen. Mit Thompson (2008) wurde kontingenztheoretisch argumentiert, dass Modekreation technisch und von Umwelteinflüssen gepuffert betrieben wird. Die Anpassung der Organisationsstruktur ermöglicht eine schnelle Reaktion auf die für innovative Systeme relevanten Veränderungen in der Umwelt, ohne dass der Kernbetrieb tangiert wird. Diese strukturelle Anpassung wurde mit Mintzbergs (1989) Adhocratie-Modell erläutert und für die Modeunternehmen adaptiert und an die Bedingungen und Bedürfnisse im Feld der Mode angepasst. Dabei ist frappant, wie bestimmte organisationale Labels Individuen mit besonderen Fähigkeiten ausstatten, die infolgedessen den Grad des kreativen Ausmaßes bestimmen. Ebenso spricht das Feldmaterial in Bezug auf die Standardisierung eine deutliche Sprache: Kernaktivitäten und Prozesse finden auf routinebasierten Verfahren statt, die durchaus Veränderung und Flexibilität zulassen, sich jedoch an bekannten Musterverfahren und Prozessprogrammen ausrichten. Organisationale Kreativität resultiert folglich aus der geschickten Trennung standardisierter Verfahren und dem Wunsch nach Erneuerung aus der Umwelt. Das heißt, dass die technologischen Bedingungen trotz Einschränkungen (constraints) und Abhängigkeiten (contingencies) durch die Neuheitsroutine kontinuierlich fortgeführt werden. Die vorliegenden empirischen Fallbeispiele haben gezeigt, dass die Modefirmen die situativen Komponenten modular bedienen, modifizieren und »kompatibel mit gewünschten organisatorischen Lösungen« machen (Kieser 20I4: I83). Insofern wird die Annahme der Kontingenztheorie bestätigt, dass der Or- 
ganisationsbetrieb von situativen Faktoren abhängig ist. Routinen, Technologien und Projekte beschränken die Organisationen zwar, eröffnen ihnen aber gleichermaßen wichtige organisatorische Gestaltungsspielräume. Organisationen sind daher nicht nur umweltabhängig, sondern können ihre Situation auf der Mikroebene direkt beeinflussen; sie reagieren auf und reproduzieren die Umwelt.

Die technologischen Aspekte beschreiben, wie Kreativität bzw. Mode von flexiblen Routinen, der Machbarkeit durch definierte Techniken, Apparaturen und Wissen abhängen. Die entworfenen Ideen müssen für die Technologie übersetzt werden, damit später die serielle Produktion ermöglicht werden kann. Aufgrund der Übersetzung der externen Ideen in interne Vorstellungen wird einerseits substanzielle Veränderung der Verfahrenstechniken verhindert und dennoch (inhaltliche) Varianz zugelassen. Die Entwicklung setzt eine Kalibrierung der kreativen Vorstellungen und der technologischen Bedingtheit voraus, weshalb oft nur geringe Dosen des künstlerischen Vorhabens realisierbar sind. Die Realisierung der kreativen Ideen findet mittels zweierlei Fertigungstechniken statt, die sich an je einem untersuchten Standort spiegeln: Der Generalist produziert die Modekollektionen anhand der Werkstattfertigung, während der Spezialist die Kleider nach dem Fließprinzip fertigt. Die empirischen Beispiele der Partnerschaft und der Strickmaschine veranschaulichen, wie Organisationen in der Entwicklung von neuen Kollektionsmodellen betriebliche Technologien vor unzähligen technologischen Alternativmöglichkeiten puffern. Trotz vestimentärer Neuerungen (thematische Inspirationen, Modellskizzen und Entwurfsmodelle) beschränkt sich die Kreativitätshervorbringung auf das Wissen und die Fähigkeiten der Partner und die Techniken der Maschine. Technologie meint folglich mehr als nur eine zuverlässige und langfristige Zusammenarbeit auf Vertrauensbasis (Partnerschaft mit Produzenten) oder Verlässlichkeit (Technikbezug zur Stickmaschine). Das Verhältnis kennzeichnet eine gegenseitige Abhängigkeit, denn die Modefirmen sind auf die handwerkliche, technische Expertise der Produzenten sowie der Maschine angewiesen, was bewirkt, dass die Produktionsstätten, deren Know-how und Infrastruktur vor jeglichen Umwelteinflüssen, temporären Turbulenzen oder Störungen abgeschottet werden. ${ }^{73}$ Insofern lösen technologische Bedingungen Machbarkeitsfragen ein, denn Machbarkeitsentscheidungen werden an der Technologie ausgerichtet. Demnach haben Technologien einen großen Einfluss auf die Kollektionsentwicklung und im Spezifischen auf die technologische Umsetzung und Dosierung des kreativen Vorhabens.

73 | Während die Maschine nicht von den Auftraggebern abhängig ist, ist die Abhängigkeit zwischen Produzent und Modefirma zweiseitig: während die Partner regelmäßig Aufträge erhalten und so den Umsatz sichern, ermöglichen der enge Kontakt und die Loyalität direkte Kontrolle durch die Modefirma. 
Als Beispiel hat sich gezeigt, wie strukturelle Bedingungen von Organisationsmitgliedern (v. a. der kreativen Leitung und dem Designteam) als Bestandteil ihrer persönlichen (Design-)Aufgabe inkorporiert werden, um unter den existierenden Bedingungen Kreatives zu schaffen. Technologien finden sich in der alltäglichen Arbeit wieder, z. B. wenn die Designerin ihre Entwürfe so skizziert, dass sie für die Strickmaschine umsetzbar sind, oder Nuancenunterschiede einer Farbe innerhalb der gleichen Kollektion akzeptiert werden. Als Folge dessen wird die kreative Leitung mit der Übersetzung betraut.

Die Übersetzung fungiert als Kontrollmechanismus des Umwelteinflusses auf den Produktionsprozess, denn die aus der Umwelt stammenden kreativen Ideen, Trends und Moden werden vom Betriebskern als Störung eingestuft, da sie unbekannt sind und Diskontinuität im Produktionsprozess anregen. Der Entstehungsprozess von Kreativität erfolgt nicht linear, sondern als dynamisch-rekursive Entwicklung, die sich zwischen Kompromiss und Konservation bewegt. Erneuerungen in der Mode tangieren darum lediglich einzelne Aspekte des Designs wie Farbwahl, Druckmuster oder schnitttechnische Modifikationen. Porac u. a. (I989) haben die Differenzierungsvarianten als Möglichkeit herausgearbeitet, anhand derer Firmen derselben Nische ihre Verschiedenheit deutlich machen können. Die Autoren sprechen vom »konkurrenzfähigen Scheitelpunkt«:

The belief of many managers is that the best differentiating strategy is to offer design variations on the classic theme that are more attractive than the variations produced by other firms, and to do so in a way that maximizes a retailer's ability to customize. Thus, the apparent differences among Scottish firms have to do with styling, colour and subtle variations in technology that are believed to give a firm a competitive edge. In this way, differences are created within an otherwise homogenous competitive cusp. (Porac u. a. 1989: 414)

Die wettbewerbsfähigen Modifikationen sind stilistische Erneuerungen, keine absoluten Neuerungen, die sich vor allem erfahrene Modefirmen zu Nutzen machen können, denn einerseits besitzen sie schon ein eigenes »classic theme «, eine Handschrift, andererseits kennen sie die competitive edge, deren Formgestaltung zentral für die Kollektionsneuerungen sind. Schließlich signalisieren die stilistischen Modifikationen nicht nur die Kompetenz kreativer Erneuerung, sondern kreieren auf der Basis gestalterischer Variationsfähigkeit individuelle Attribute, die schwer zu imitieren sind. Nichtsdestotrotz werden Virtuosität und Neuinterpretationen der alten, traditionellen Muster als Eigenschaften der Erneuerungen institutionell (mit-)erwartet. Dazu werden entsprechende Fassaden aufgebaut, die durch zeremonielle Formen aufrechterhalten werden und den institutionalisierten Kreativitätsmythos pflegen. Diese legitimierenden Kreativitätszeremonien und Neuerungsnarrative werden unter den 
kommunikativen Aspekten der Kreativität detailliert beschrieben (s. Kap. 7 bzw. 8.2.3). Die Neuheitsroutine und insbesondere deren stilistische Modifikationen tragen daher zur Ausdifferenzierung im Feld der Mode bei.

Zum Zweck der Koordinierung dieser Vorgänge hat sich die projektbasierte Organisationsform als tragfähiges Konzept herausgestellt, die permanente Strukturen (Hierarchie, technologische Voraussetzungen) mit temporären Ressourcen (professionelles Expertenwissen, Apparaturen und Techniken) kombiniert. Das Projekt ist eine permanent-temporäre Organisationsform, in der Zeit (zeitlicher Druck) und Wissen (spezialisiertes Know-how) die Bedeutung im Schaffens- und Entstehungsprozess der Modekollektion fundamental beeinflussen. Auf der Basis von Projekten koordinieren Modeunternehmen sowohl bürokratische Strukturen, die sich vor allem in den Anstellungsverhältnissen und der Organisation des Projekts zeigen, als auch temporäre Arbeitsstrukturen, die sich im zeitlich begrenzten Zusammenarbeiten sowie in den Projektinhalten (Ziele, Aufträge, Mitglieder, Zeithorizont, Budget) offenbaren. Projekte verbinden die Organisation mit der Umwelt, da sie oft - zumeist in der Modebranche - unter der Schirmherrschaft einer ständigen Formalstruktur (Unternehmen) stattfinden, jedoch temporär beschränkt existieren und während der Projektlaufzeit aus einem »bunten Haufen« verschiedener Mitglieder bestehen. Die temporär angeheuerten Teilnehmer lösen die Prominenz der formalen Organisation auf. Aus dem Bruch mit organisationalen Grenzen und Konventionen resultiert ein Tummelplatz für neue Ideen und originelle Einfälle.

Weiter koordinieren die projektbasierten Kollektionsphasen und Arbeitsteams die Interdependenz und Verkettung der lose gekoppelten Ebenen der Aktivitäts- und der Formalstruktur. In den einzelnen Projekten wird darüber befunden, zu welchem Grad die Technologie bewahrt bzw. der Variation stattgegeben wird. Diese Mikroprozesse haben einen großen Einfluss auf die Institutionalisierung in den Organisationen, denn sie bedingen, ob eine Praktik zur Routine wird oder Bestandteil der veränderlichen Fassadengestaltung bleibt. Für die Unternehmen bedeutet dies, dass auf der operativen Ebene entschieden wird, ob textile Techniken, Inspirationen, Ideen oder Trends sowie deren gestalterische Umsetzung als Kollektionsmodell Bestandteil einer Kollektion bleiben oder zu einem »Evergreen « oder »Klassiker« avancieren. Kreativität in der Mode ist somit ein Termingeschäft und das Resultat einer modularen Aktivitätsstruktur sowie ihrer Terminierung.

Das Kreieren einer neuen Modekollektion ist somit kein einmaliges, alleinstehendes Vorhaben, sondern ein in eine bestehende Formalstruktur integriertes Unterfangen. Projekte fördern den Zufluss und Austausch nicht redundanter Ressourcen, da sie Kontakt zwischen permanenten, ehemaligen und »neuen« Mitgliedern herstellen. Während der Projektlaufzeit entsteht somit eine temporäre Öffnung gegenüber der Umwelt, weshalb Projekte als 
Schaltstelle zwischen Organisation und institutionellem Feld fungieren und die Informations- und Ressourcenzirkulation kontrollieren. Demzufolge fungieren Projekte als Mittel der Umweltbeobachtung, während sie gleichzeitig Instanzen der Abschottung des Kernbetriebs sind. Trotz Kooperationen mit Künstlern, Magazinen oder Experten weichen die Unternehmen selten von den technologischen Verfahren ab. Projekte sind Organisationsformen organisierter Variation, die mit einem gewissen Toleranzniveau für Wandel ausgestattet sind, wobei die Veränderung der Projektparameter (Laufzeit, Aufgabe, Teamkonstellation) auch Unvorhersehbares initiieren kann. Kontingenztheoretisch gesprochen, fungiert die Projektorganisation als Puffer. Als Instanz der Varianzkontrolle von Personal, Wissen oder technischen Fähigkeiten wird in Projekten der fremde Einfluss auf die Organisation entweder als zulässig oder als störend ermittelt. Die Machbarkeitserwägungen richten sich an technologischen Voraussetzungen aus, finden aber in bunt gemischten Projektteams statt.

Das Dilemma zwischen Öffnung und Schließung der Organisation gegenüber der Umwelt wird durch das lose Koppeln der Teilbereiche Technologie und Sprache organisatorisch gelöst. Der technologische Kern, der vornehmlich organisational gekoppelt und geschlossen ist, und die Neuheit, die im System meist lose und offen besteht, sind nur an gewissen Berührungspunkten verbunden. Da Mode einer gewissen Portion Erneuerung und Varianz bedarf, wird Kreativität im Modedesign sowohl durch die totale Öffnung (avantgardistische Designs gelten als unverständlich) als auch die totale Schließung (Kopien und Reproduktionen gelten als unmodisch) verunmöglicht. Der Berührungspunkt zwischen technologischem Kern und Neuheit wird darüber gesteuert, dass Neuerungen in der Umwelt registriert und von der Organisation übernommen werden, um dann an die technologischen Bedingungen angepasst zu werden. Der Technologiefokus kontrolliert das Ausmaß, zu welchem die Neuerung, die nicht nur zur Wettbewerbsfähigkeit, sondern auch zur kreativen Glaubwürdigkeit beiträgt, umgesetzt bzw. adaptiert wird. Die Neuheitsroutine macht Veränderung und Erneuerung möglich, während die Aktivitätsbasis das Bewährte und Beherrschte bleibt. Dadurch wird Kreativität trotz Kontinuität möglich. Die Neuheitsroutine ist das Resultat der losen Kopplung zwischen Formal- und Aktivitätsstruktur. Sie ist die technologische Antwort auf das Dilemma der Öffnung und Schließung der Aktivitätsstruktur, die zwar die Basis der konstanten Weiterentwicklung bleibt, durch die Neuheitsroutine aber partiell und temporär verändert wird.

\subsubsection{Kommunikative Bedingungen und Fragen der Darstellung}

Empirisch konnte ermittelt werden, dass Organisationen mit Narrativen und Inszenierungen auf institutionelle Erwartungen der Umwelt reagieren, so 
auch auf bestehende gesellschaftliche Kreativitätsmythen. Zeremonien sind die Reaktion auf die technologische Fertigung im Betriebskern und Praktiken, Prozesse, Personen und Produkte »re-mystifizieren« bzw. »kreativisieren« diese Fertigungsverfahren. Sprachliche und zeremonielle Ausgestaltungen ergänzen die von Technologie, Zeit und Kommerzialität geprägte Entscheidungsfindung und konstruieren eine individuelle, kreative Fassade und bestätigen hierbei den Glauben an Kreativität als etwas Fundamentales. Diese »Kreativisierung « findet in der Formalstruktur der Organisation statt, die Kreativität sprachlich ausweist und verankert, hat aber gleichermaßen einen Effekt auf die technologische Produktion der Güter, die währenddessen im »Hintergrund « kontinuierlich weiteroperiert. Die Modefirmen haben damit eine Möglichkeit geschaffen, trotz Routinen, Technologien und Wiederholungen Kreativität zu reklamieren. Die Aktivitätsstruktur hingegen wird organisational gepuffert und ist nur lose mit den kreativen Glaubenssätzen und Fassaden gekoppelt. Die Analyse des Feldmaterials zeigt, dass Sprache und Zeremonien hierbei von Bedeutung sind. Eigene Sprachspiele, aber auch Erzählungen von Drittparteien liefern die evaluative Basis für Zuschreibungen und Bewertungen der >kreativen $<$ Organisation. Sprachspiele - damit sind sowohl rhetorische als auch visuelle Inszenierungen gemeint - signalisieren die Bedeutung der Mikroprozesse für die unternehmerischen Darstellungen der Vergleichbarkeit und Unvergleichbarkeit kreativer Güter bzw. der Feld- und Nischenzugehörigkeit der Unternehmen. Damit wird angesprochen, was in dieser Arbeit als die kommunikativen Aspekte von Kreativität begriffen wird.

Die konstruktive Praxis der kommunikativen Aspekte bezieht sich maßgebend auf Darstellungsformen. Institutionelle Erwartungen schmücken die Mode mit der Annahme, sie sei neu und kreativ, ohne dass genauer bekannt ist, was Mode ist und wie sie entsteht. Im Modesektor reproduzieren Kreativitätszeremonien und Neuerungsnarrative den Glauben daran, dass die regelmäßige Abänderung bestehender und Berücksichtigung fremder Deutungen, wie Trends, Ideen und Moden, die rationale Grundlage der Erneuerung der Mode sind. Das Beobachten, Übersetzen und Adaptieren von Inspirationen durch eine kreative Person oder ein Team sind an dieser Stelle die adäquaten Methoden der Modekreation. Diese Praxis wird weder hinterfragt noch untergraben. Die saisonale Erneuerung der Mode wird als natürliche Ordnung der Modekreation betrachtet, die zur Reproduktion der Kreativitätsmythen und zur Institutionalisierung der Mode beiträgt. Es ist die Aufgabe der sozialen Akteure, die materiellen und symbolischen Institutionen kontinuierlich und wiederholt herzustellen: »It is the continuous enactment of practices and meanings by organization members that constitutes and maintains institutions, including their appearance and experience as taken-for-granted « (Zilber 2002: 25I). Als Folge davon werden über kollektionsspezifische Hinweise Geschichten konstruiert, die dann in Shows der Kreativität und durch Storytelling dargestellt 
werden. Mittels Kreativitätszeremonien und Neuerungsnarrativen werden eine besondere Rhetorik und Inszenierungen der kreativen Fassade der Modefirma gepflegt.

Auf institutionellen Vokabularen bauen Narrative auf, welche die Organisation als notwendig und rational darstellen. Institutionelles Vokabular und Jargon suggerieren kreativen und künstlerischen Reichtum, dem eine besondere Außenwirkung zukommt. Es handelt sich dabei um eine symbolische Anpassung, wodurch Isomorphie mit institutionellen Erwartungen justiert und Legitimität übertragen wird. Bei diesen symbolischen Ausgestaltungen handelt es sich um Darstellungsformen, die eine aufgrund der standardisierten Verfahren entstandene Lücke zwischen effektiver Realisierung und erwarteter Kreativitätsvorstellung überbrücken und Glaubwürdigkeit wie auch Legitimität reklamieren. »Here, the term >symbolic $<$ means that the practice sends a signal to relevant audiences; it does not mean that planning [oder Darstellungsformen wie Geschichten und Inszenierungen] is superficial or inconsequential« (Bromley/Hwang/Powell 20I2: 482). Der Neoinstitutionalismus versteht Legitimität als grundlegende Bedingung für wirtschaftlichen Erfolg, den Zugang zu Ressourcen und organisationales Überleben. Die gestalterische Aufbereitung und Darstellung sind Mittel der situativen Einflussnahme von Organisationen auf die Umwelt und sind als solche als Möglichkeit der Beeinflussung und Reaktion auf Kontingenzen zu verstehen.

Die kommunikativen Kreativitätsaspekte beschreiben, wie Organisationen das relevante Publikum vom Neuheitsgehalt der Kollektion überzeugen und den Status eines kreativen und modischen Unternehmens aufrechterhalten. Geschichten wirken demzufolge als Möglichkeit der Erwartungskonstruktion sowie Rechtfertigungs- oder Reparationsstrategie. Dazu wird während der Kreativitätshervorbringung sukzessive eine Geschichte konstruiert, die auf einem vordefinierten Thema gründet und materiell und symbolisch in die Kollektion eingearbeitet wird. Zum Abschluss der Kollektionsherstellung wird diese als intendiert präsentiert und zum Zweck der Außenkommunikation rhetorisch zurechtgelegt. In Shows wird diese narrative Idee aufgegriffen und auf sehr aufwendige Weise visualisiert, indem Outfits, Accessoires und Models an ausgewählten Schauplätzen inszeniert werden. Schließlich werden die narrativen Fragmente mithilfe von Storytelling in Etiketten und Labels, Broschüren und Newslettern zu einer kohärenten Erzählung finalisiert und der Laden entsprechend in Szene gesetzt. Geschichten werden zu Biografien, z. B. zur Ausbildung der »kreativen Köpfe« oder in Form der Entstehungsgeschichte des kreativen Gegenstandes, durch Storytelling durch die Beteiligten selbst, das organisationale Feld oder die Medien erzählt. In diesen Geschichten wird auf die Einmaligkeit verwiesen und die Neuerungen der Kollektion werden herausgestrichen. Narrative Fragmente der Orientierung, Inszenierung und Finalisierung sind Formen der zeremoniellen Ausgestaltung der institutionel- 
len Erwartungserfüllung und formen sich erst zum Schluss zu einem rhetorischen Sprachspiel zwecks außengerichteter Kommunikation.

Durch die narrativen Fragmente wird die Kollektion in die Biografie der Organisation eingebettet. Dabei wird sie als minimal verschieden vom organisationalen Stil (Identität) dokumentiert und als maximal verschieden von Moden im Feld ausgewiesen (Alterität). Die Neuerung wird dann im eigenen Karriereverlauf sowie im Vergleich mit aktuellen Konkurrenten platziert, wobei ein Mittelweg zwischen der »affirmation of difference « (Czarniawska 2008) - der Verschiedenheitsbekundung - und der »range of acceptability « (Deephouse I999) - der Ähnlichkeitserklärung - gefunden werden muss. Für die Modefirmen liegt die optimale Verschiedenheit zwischen der Herausbildung eines exklusiven Stils und der Verwendung eines konsumentenfreundlichen Designs. Die Modefirmen versuchen durch die Narrative eine bestimmte Identifikation zu erzeugen, mit welcher sie ihre Nischenzugehörigkeit und Anpassung an institutionelle Erwartungen im Feld signalisieren.

Die eigenen Alleinstellungsmerkmale haben ihren Ursprung in der kognitiven und mentalen Konstruktion potenzieller Konkurrenzgruppen. Aufgrund dieser Unverwechselbarkeit - diese Nische wird von keiner weiteren Organisation bedient - erscheinen die Modeunternehmen kreativ, in dem was sie tun. »Indeed, the characteristics of stylistic innovation drive the creation of market niches where those companies that do not adopt the convergent style or do not exclusively focus on that trend, might also come out to be stylistically successful« (Cappetta/Cillo/Ponti 2006: I286). Die Nischenbildung wird von stilistischen Modifikationen vorangetrieben, wenn Unternehmen die gestalterische Umsetzung der Trends frei wählen oder sie partiell ignorieren. Wie die empirischen Beispiele zeigen, fördern der Fokus auf Klassiker und die stilistische Modifikation bewährter Kollektionsmodelle die Fundierung einer eigenen Marktnische. Weiter ist auch die Art und Weise kreativ, wie sie es tun, denn im Modedesign wird Kreativität in Form aufwendiger Darstellungen deutlich gemacht, ohne selbst zu behaupten, dass es sich um Kreativität handelt.

Die Nische wird nicht nur durch die Wahl einer bestimmten Technologie bestimmt, sondern auch durch eine Semantik, die zum kognitiven Kriterium für die Definition von Konkurrenz und Zugehörigkeit wird. Modefirmen greifen auf unterschiedliche kognitive Konstruktionen (Identität und Alterität) sowie Semantiken (Verschiedenheitsbekundungen und Ähnlichkeitserklärungen) zurück, was zumal in kleinen Märkten wie der Schweizer Modebranche als kognitive Bewältigung starken Wettbewerbs funktioniert. Aufgrund der kognitiven und mentalen Kategorisierungen, die sich in der unterschiedlichen Selbstdarstellung der beiden Unternehmen widerspiegeln, nehmen sich der spezialisierte Strickhersteller und das breit aufgestellte Traditionsunternehmen nicht als direkte Konkurrenz wahr. Diese Arbeit versucht ein Nischenverständnis zu zeichnen, das nicht nur einen bestimmten marktwirtschaftlichen 
Vorteil meint, sondern Hervorbringungsaspekte offenlegt, die Kreativität provozieren. Die Nischenfindung und -ausgestaltung wird dabei selbst zur kreativen Aktivität und die Darstellung dieser Unverwechselbarkeit reproduziert den (externen und internen!) Glauben daran.

Eine für die Variationsfähigkeit der Organisation und deren kreative Außenwirkung fundamentale Aufgabe ist es folglich, neue Ideen und potenzielle Inspirationsquellen in der Umwelt zu registrieren und diese in Form von narrativen Fragmenten in die Organisationskultur des Modeunternehmens zu übersetzen. In der Mode stammen thematische Narrative meist aus Erlebnissen der Organisationmitglieder oder aus gesellschaftlichen Diskursen, womit gegenseitige Wahrnehmung und institutionelle Zugehörigkeit signalisiert werden. Diese Ideen entstammen den »Meta-Narrativen « der institutionellen Umwelt, aus der sie von der kreativen Elite - in diesem Fall durch die Creative Directors - in den lokalen Kontext übersetzt und individuell modifiziert werden: »Rather, through interpretation organizational elites translate them into local - more specific and selective - versions, which are then used in orgnaizational sensemaking processes « (Zilber 2009: 206). Die Translation und das Editieren von Meta-Narrativen in den lokalen Kontext statten die Unternehmen mit institutionellen, passenden Argumentationsbausteinen aus und erzeugen damit organisationale Historien, Biografien und Identitäten. Um modische Erneuerung reklamieren zu können, müssen etablierte narrative Fragmente offensichtlich transformiert werden. Insofern können Narrative und deren lokale Deutungen institutionellen Wandel fördern. Werden diffundierte Trends und Moden von einer Firma übernommen, so werden sie mit den existierenden technischen Mitteln in die lokale Technologie übersetzt und kommunikativ in die organisationale Kultur, d. h. die aktuelle Kollektion, eingebettet. Eine neue Idee wird als narratives Fragment in die Organisation übernommen, wo es eine sprachliche Verbindung zwischen den dauerhaften Institutionen (z. B. der organisationalen $»$ Handschrift«) und den vorübergehenden Modeerscheinungen (z. B. Modetrends) herstellt. Diese Übersetzung macht die fremde Idee in der Organisation anschlussfähig. Demnach ist Modedesign ein Arrangement zwischen existierenden Institutionen und verfliegenden Moden. Die Wirksamkeit zeremonieller Regeln und institutioneller Erwartungen - in diesem Falle die Adaption zirkulierender, institutionalisierter Moden - zeigt sich darin, dass die Ablehnung reale Konsequenzen für die Organisation haben kann. Institutionelle Anpassung an die Modeumwelt gewährleistet Legitimität und Ressourcen, stabilisiert die Kreditwürdigkeit, demonstriert soziale Fitness und erhöht die Überlebenschancen. Die Darstellungsformen der Mode sind sowohl geplante wie auch emergente Konstruktionen und Reproduktionen institutionalisierter Kreativitätsmythen. Anhand von unterschiedlichen Darstellungsformen wird die Kollektion institutionell und organisational passend 
gemacht. Kreativitätszeremonien und Neuerungsnarrative rekonstruieren die »Kreativisierung«.

\subsection{Schlussbemerkungen und Ausblick}

Im Untersuchungsfokus der vorliegenden Arbeit standen die Wechselwirkung zwischen der institutionellen Umwelt und der Organisation und ihrem technologischen Kern, deren Analyse eine Erklärung für die technologischen Bedingungen der Produktion und kommunikative Darstellung von Kreativität aus organisationssoziologischer Perspektive ermöglichen sollte. Die Bausteine werden wie folgt zusammengefasst: Die kulturpolitische Einführung in das Thema der Creative Industries in Kapitel 3 und die kulturtheoretische, historische Beschreibung der Entstehung und Verwendung des Kreativitätsbegriffs in verschiedenen wissenschaftlichen Disziplinen in Kapitel 4 repräsentieren die konzeptuelle Annäherung an den Untersuchungsgegenstand. Um die Vorgehensweise zu explizieren, wurde das Forschungsdesign der empirischen Untersuchung zu Beginn vorgestellt. Dies mit der Absicht, auf die Verwendung des Begriffs Kreativität im Feld hinzuweisen.

Die empirischen Beobachtungen haben mich dazu geleitet, das Phänomen der Kreativitätshervorbringung mit Konzepten aus dem soziologischen Neoinstitutionalismus als auch der Kontingenztheorie $\mathrm{zu}$ theoretisieren, die in Kapitel 6 und 7 jeweils erläutert und mit Beispielen aus dem Feldmaterial ergänzt wurden. Diese Theoretisierung ist ein Versuch, die Beobachtung geschickt mit Erklärungen zu verknüpfen. Darauf aufbauend wurde die Kreativitätshervorbringung in den zwei Betrieben analysiert, die auf den ersten Blick verschiedener wirken mögen, als dass sie schließlich sind. In diesem Sinn und in Anlehnung an Everett C. Hughes wurde die »likeness inside the shell of variety« fokussiert (Hughes I993: 503). Dieses Vorgehen erlaubte, das Phänomen Kreativität in zwei Teilkonzepte aufzuteilen: die technologischen und die kommunikativen Aspekte der Kreativitätshervorbringung. Zunächst wurden die technologischen Bedingungen der Produktion der kreativen Güter beschrieben, um daran anschließend die kommunikativen Voraussetzungen der Darstellung von Kreativität zu erörtern. Für beide Kapitel gilt, dass erst die als notwendig erachteten theoretischen Konzepte vorgestellt und dann mit empirischen Beispielen unterfüttert wurden.

Das Kapitel hat sich mit den zentralen Ergebnissen der Arbeit auseinandergesetzt, die wie folgt zusammengefasst werden können: In Übereinstimmung mit der Argumentation der Kontingenztheorie legen technologische Bedingungen durchaus eine Spezialisierung auf bestimmte technische Möglichkeiten nahe, die auch für die Modeindustrie feststellbar ist. Sowohl die Spezialisierung als auch die Generalisierung der kreativen Güter sind als Strategie 
der technologischen Wahrung und produktiven Kontinuität zu verstehen. Jedoch reicht das Argument nicht aus, dass Kreativität lediglich auf Routinen, Technologien und Projektarrangements basiert. In Übereinstimmung mit der Argumentation des Neoinstitutionalismus konnte festgestellt werden, dass zeremonielle und sprachliche Hinweise an Bedeutung gewinnen, denn mittels dieser Darstellungen gelingt es den Modefirmen, trotz standardisierter Produktionsverfahren Kreativität zu reklamieren. Diese Arbeit bestätigt demnach die kollektive und organisationale Herstellung von Kreativität und fügt diesem Verständnis eine institutionelle Dimension hinzu, die besagt, dass Kreativität ein Label ist, dass sowohl sozial konstruiert wie auch zugeschrieben wird. Die technologischen Kreativitätsaspekte setzen sich aus der technologischen Einschränkung, den routineähnlichen Verfahren und der projektbasierten Koordinationsform zusammen und werden durch die kommunikativen Kreativitätsaspekte komplettiert, die sich aus Narrativen, Shows und Storytelling zusammenfügen. Ein Abgleich dieser beiden Eigenschaften von Kreativität hat gezeigt, wie diese entsteht: Einerseits werden durch die Neuheitsroutine Inhalte (Inspirationen, Muster, Schnitte, Stoffqualitäten etc.) angeeignet und andererseits durch die Kreativitätszeremonien verfremdet und durch Neuerungsnarrative aktualisiert. Dadurch, dass Modekreation ein lose gekoppelter Übersetzungsprozess von fremden Ideen in technologische Machbarkeit ist, sind Modefirmen kreativ, indem was sie machen. Dadurch, dass Modekreation durch diese Übersetzung institutionelle, globale Erwartungen mit organisationalen, lokalen Vorstellungen kombinieren und erneuern, sind Modefirmen kreativ indem, wie sie es machen. Während Ersteres Isomorphie begünstigt und die institutionelle Passung der Modefirme fokussiert, ermöglicht Letzteres die institutionelle Ausdifferenzierung und kognitive Nischenbildung.

Die technologisch-kommunikativen Eigenschaften organisationaler Kreativität erlauben es, Bezüge zu verschiedenen Subsektoren im Feld der Mode als auch zu anderen Kreativindustrien herzustellen.74 Eine weitere, zu verfol-

74 | So ähnelt die Modebranche beispielsweise der Gastronomie, denn beide Branchen bringen Neuheiten primär durch Variationen, d. h. inkrementelle Variationen statt radikalen Neuerungen, hervor. Die Außendarstellung der Mode unterscheidet sich jedoch weitgehend von derjenigen der Gastronomie, die sich auf die kulinarische, also geschmackliche und optische Kreation, Nennungen in Gastronomieführern oder Auszeichnungen von Küchen und Köchen bezieht. Demgegenüber ist die Filmbranche der Modeindustrie näher, denn Filmfestivals, Awards und Auszeichnungen sowie Trailer können als Selbstinszenierung einer auf (breiter) Außenwirkung beruhenden Industrie verstanden werden. Im Film finden radikale Innovationen direkte Anwendung (3 D, Streaming), was die Filmindustrie von der Mode unterscheidet. Des Weiteren löst sich ein Filmset nach Beendigung der Dreharbeiten in seine Einzelteile auf; die Mode jedoch bewirtschaftet ein "Mutterhaus", das permanent operiert. 
gende Hypothese lautet demnach, dass sich die Einstellungen »Technologie« bzw. »Kommunikation « in Form eines graduellen Reglers auf unterschiedliche marktwirtschaftliche oder institutionelle Bedürfnisse anwenden lassen. Vergleicht man kommerziell ausgerichtete Bekleidungshersteller mit den künstlerischen Ausrichtungen von Couture-Häusern, so werden fundamentale Unterschiede deutlich. Während die Konfektionsbekleidung stark über technologische Bedingungen geregelt und kaum kommunikativ ausgewiesen wird, kann vermutet werden, dass Couture stark über kommunikative Darstellungen reklamiert und die technologischen Voraussetzungen als sekundär betrachtet werden.

Diese Arbeit stellt fest, dass die hergebrachten Definitionen von Kreativität in der Mode unzureichend sind. Als projektbasierte Organisationsform beruht Mode auf technologischen Eingrenzungen und routinierten Verfahren und schafft sich somit einen Raum der Gleichzeitigkeit von Beständigkeit und Variation, Tradition und Erneuerung, serieller Herstellung und künstlerischer Kreation. Folglich können nur mehrdimensionale Beschreibungen das Phänomen Kreativität erklären. Dieses Buch mag die Entwicklung und Darstellung der Modekreation zwar demystifizieren, es schafft es aber nicht, meine Faszination am Gegenstand Mode zu verringern. 


\section{Bibliografie}

Aage, Tine/Belussi, Fiona (2008): From Fashion to Design: Creative Networks in Industrial Districts. In: Industry \& Innovation I5 (5), 475-49I.

Aldrich, Howard E./Fiol, C. Marlene (I994): Fool Rush In? The Institutional Context of Industry Creation. In: Academy of Management Review I9 (4), $645-670$.

Alvarez, José Luis/Mazza, Carmelo/Strandgaard Pedersen, Jesper/Svejenova, Silviya (2005): Shielding Idiosyncrasy from Isomorphic Pressures: Towards Optimal Distinctiveness in European Filmmaking. In: Organization I2 (6), 863-888.

Alvesson, Mats/Kärreman, Dan (2000): Taking the Linguistic Turn in Organizational Research. In: The Journal of Applied Behavioral Science 36 (2), I36-I58.

Amabile, Teresa M. (I982): Social psychology of creativity: A consensual assessment technique. In: Journal of Personality and Social Psychology 43 (5), 997-IOI3.

Amabile, Teresa M. (I983): The social psychology of creativity: A componential conceptualization. In: Journal of Personality and Social Psychology 45 (2), $357-376$.

Amabile, Teresa M. (I995): Commentary: Attributions of Creativity - What Are the Consequences? In: Creativity Research Journal 8 (4), 423-426.

Amabile, Teresa M. (I996): Creativity in Context. Colorado/Oxford: Westview Press.

Amabile, Teresa M. (I998): How to Kill Creativity. In: Harward Business Review $76(5), 76-87$.

Amabile, Teresa M./Conti, Regina/Coon, Heather/Lazenby, Jeffrey/Herron, Michael (I996): Assessing the Work Environment for Creativity. In: Academy of Management Journal 39 (5), II54-II84.

Anand, N./Peterson, Richard A. (2000): When Market Information Constitutes Fields: Sensemaking of Markets in the Commercial Music Industry. In: Organization Science II (3), 270-284.

Andriopoulos, Constantine (200I): Determinants of organisational creativity: a literature review. In: Management Decision 39 (Iо), 834-84I. 
Ashforth, Blake E./Humphrey, Ronald H. (I997): The Ubiquity and Potency of Labeling Organizations. In: Organization Science 8 (I), 43-58.

Aspers, Patrik (20I0): Orderly fashion. A sociology of markets. Princeton/Oxford: Princeton University Press.

Aspers, Patrik/Godart, Frédéric (2013): Sociology of Fashion: Order and Change. In: Annual Review of Sociology 39, I7I-I92.

Bakker, René M. (2010): Taking Stock of Temporary Organizational Forms: A Systematic Review and Research Agenda. In: International Journal of Management Reviews I2 (4), 466-486.

Bakker, René M./Boroş, Smaranda/Kenis, Patrick/Oerlemans, Leon A. G. (20I3): It's Only Temporary: Time Frame and the Dynamics of Creative Project Teams. In: British Journal of Management 24 (3), 383-397.

Bakker, René M./DeFillippi, Robert J./Schwab, Andreas/Sydow, Jörg (2016):

Temporary Organizing: Promises, Processes, Problems. In: Organization Studies 37 (13), I703-I719.

Barrett, Frank J. (I998): Creativity and Improvisation in Jazz and Organizations: Implications for Organizational Learning. In: Organization Science 9 (5), 605-622.

Bartel, Caroline A./Garud, Raghu (2009): The Role of Narratives in Sustaining Organizational Innovation. In: Organization Science 20 (I), IO7-II7.

Barthes, Roland (I986): Rhetorik des Signifikats: Die Welt der Mode. In: Silvia Bovenschen (Hg.): Die Listen der Mode. Frankfurt am Main: Suhrkamp, 29I-308.

Bechky, Beth A. (2006): Gaffers, Gofers, and Grips: Role-Based Coordination in Temporary Organizations. In: Organization Science I7 (I), 3-2I.

Becker, Howard S. (I974): Art as a Collective Action. In: American Sociological Review 39 (6), 767-776.

Becker, Howard S. (2008): Art Worlds. 2. Aufl. Berkley/Los Angeles: University of California Press.

Becker, Markus C. (2008): The Past, Present and Future of Organizational Routines: Introduction to the Handbook of Organizational Routines. In: Ders. (Hg.): Handbook of Organizational Routines. Cheltenham (UK)/ Northampton (MA): Edward Elgar, 3-I4.

Beckert, Jens (20II): The Transcending Power of Goods: Imaginative Value in the Economy. In: Ders./Patrik Aspers (Hg.): The Worth of Goods: Valuation and Pricing in the Economy. Oxford: Oxford University Press, Io6-I28.

Berger, Peter/Luckmann, Thomas (I99I): The Social Construction of Reality - A Treatise in the Sociology of Knowledge. 6. Aufl. Austin (TX): Penguin Social Sciences.

Berger, Peter L./Luckmann, Thomas (Hg.; 20I3): Die gesellschaftliche Konstruktion der Wirklichkeit. 25. Aufl. Frankfurt am Main: Fischer. 
Bilton, Christ (2007): Managment and Creativity: From Creative Industries to Creative Management. Malden: Blackwell.

Blumer, Herbert (I969): Fashion: From class differentiation to collective selection. In: The Sociological Quarterly Io (3), 275-29I.

Boden, Margaret A. (2005): The Creative Mind. 2. Aufl. New York: Routledge. Bohn, Cornelia (2006): Kleidung als Kommunikationsmedium. In: Dies. (Hg.): Inklusion, Exklusion und die Person. Konstanz: UVK Verlagsgesellschaft, 95-I24.

Boje, David M. (I99I): The Storytelling Organization: A Study of Story Performance in an Office-Supply Firm. In: Administrative Science Quarterly 36 (I), 106-I26.

Boje, David M. (I995): Stories of the Storytelling Organization - A Postmodern Analysis of Disney As Tamara-Land. In: Academy of Management Journal 38 (4), 997-I035.

Boje, David M. (Hg.; 200I): Narrative Methods for Organizational \& Communication Research. London/Thousand Oaks: SAGE Publications.

Boland, Richard J./Tankasi, Ramkrishnan V. (I995): Perspective Making and Perspective Taking in Communities of Knowing. In: Organization Science $6(4), 350-372$.

Bornemann, Stefan (20I2): Kooperation und Kollaboration. Das Kreative Feld als Weg zu innovativer Teamarbeit. Wiesbaden: Springer VS.

Bourdieu, Pierre (I993): But Who Created the >Creator<? In: Ders.: Sociology in Question. London/Thousand Oaks: SAGE Publications, I39-I48.

Bouty, Isabelle/Gomez, Maire-Léandre (20I6): Creativity at Work. In: Raghu Garud u. a. (Hg.): The Emergence of Novelty in Organizations. Oxford: Oxford University Press, 216-24I.

Brady, Tim/Davies, Andrew (2004): Building Project Capabilities: From Exploratory to Exploitative Learning. In: Organization Studies 25 (9), I6oI-I62I. Bromley, Patricia/Hwang, Hokyu/Powell, Walter W. (2012): Decoupling revisited: Common pressures, divergent strategies in the U.S. nonprofit sector. In: M@n@gement I5 (5),468-5or.

Brown, Andrew D./Gabriel, Yiannis/Gherardi, Silvia (2009): Storytelling and Change: An Unfolding Story. In: Organization I6 (3), 323-333.

Brown, John Seely/Duguid, Paul (I99): Organizational Learning and Communities of-Practice: Toward a Unified View of Working, Learning, and Innovation. In: Organization Science 2 (I), 40-58.

Burow, Olof-Axel (I999): Die Individualisierungsfalle: Kreativität gibt es nur im Plural! Stuttgart: Klett-Cotta.

Burt, Ronald S. (2004): Structural Holes and Good Ideas. In: The American Journal of Sociology iıo (2), 349-399. 
Cappetta, Rossella/Cillo, Paola/Ponti, Anna (2006): Convergent designs in fine fashion: An evolutionary model for stylistic innovation. In: Research Policy 35 (9), I273-I290.

Carlsen, Arne/Clegg, Stewart/Gjersvik, Reidar (20I3): Idea Work. Lessons of the Extraordinary in Everyday Creativity. 2. Aufl. Oslo: Cappelen Damm Akademisk.

Catmull, Ed/Wallace, Amy (20I4): Creativity, Inc. Overcoming the Unseen Forces that Stand in the Way of True Inspiration. New York: Randhom House.

Cattani, Gino/Ferriani, Simone (2008): A Core/Periphery Perspective on Individual Creative Performance: Social Networks and Cinematic Achievements in the Hollywood Film Industry. In: Organization Science I9 (6), $824-844$.

Cattani, Gino/Ferriani, Simone/Colucci, Mariachiara (20I5): Creativity in Social Networks. In: Candace Jones/Mark Lorenzen/Jonathan Sapsed (Hg.): The Oxford Handbook of Creative Industries. Oxford: Oxford University Press, 75-95.

Caves, Richard E. (2002): Creative Industries: Contracts between Art and Commerce. 2. Aufl. Cambridge (MA): Harvard University Press.

Caves, Richard E. (20I2): Contacts Between Art and Commerce. In: Brian Moeran/Ana Alacovska (Hg.): Creative Industries: Critical Readings. London: Berg, Bd. 2: Economy, 3-I3.

Chai, Andreas; Peter Earl/Potts, Jason (20II): Fashion and Economic Evolution. In: Jason Potts (Hg.): Creative Industries and Economic Evolution. Cheltenham (UK)/Northampton (MA): Edward Elgar, II9-I27.

Chen, Katherine K. (2009): Enabling Creative Chaos. Chicago/London: The University of Chicago Press.

Chen, Katherine K. (20I2): Organizing Creativity: Enabling Creative Output, Process, and Organizing Practices. In: Sociology Compass 6 (8), 624-643. Child, John (I972): Organizational Structure, Environment and Performance: The Role of Strategic Choice. In: Sociology 6 (I), 2-22.

Child, John (I997): Strategic choice in the analysis of action, structure, organizations and environment: retrospect and prospect. In: Organization Studies I, $43-76$.

Cohen, Michael D./Burkhart, Roger/Dosi, Giovanni/Egidi, Massimo/Marengo, Luigi/Warglien, Massimo/Winter, Sidney (I996): Routines and Other Recurring Action Research Issues. In: Industrial and Corporate Change 5 (3), $653-698$.

Crane, Diana (2000): Fashion and its Social Agendas. Chicago/London: The University of Chicago Press.

Crane, Diana (20I2): Globalization, Organizational Size, and Innovation in the French Luxury Fashion Industry: Production of Culture Theory Revisited. 
In: Brian Morean/Ana Alacovska (Hg.): Creative Industries: Critical Readings. London/New York: Berg, Bd. 3: Organization, 80-Io2.

Crane, Diana/Bovone, Laura (2006): Approaches to material culture: The sociology of fashion and clothing. In: Poetics 34, 319-333.

Creed, W. E. Douglas/Scully, Maureen A./Austin, John R. (2002): Clothes make the person? The tailoring of legitimating accounts and the social construction of identity. In: Organization Science I3 (5), 475-496.

Cronin, Matthew A./Loewenstein, Jeffrey (20I8): The Craft of Creativity. Stanford: Stanford University Press.

Csíkszentmihályi, Mihaly (I988): Society, culture, and person: a system view of creativity. In: Robert J. Sternberg (Hg.): The Nature of Creativity. Cambridge/New York/Melbourne: Cambridge University Press, 325-339.

Csíkszentmihályi, Mihaly (I996): Creativity: Flow and the Psychology of Discovery and Invention. New York: Harper Collins Publishers.

Csíkszentmihályi, Mihaly (2007): Implications of a Systems Perspective for the Study of Creativity. In: In: Robert J. Sternberg (Hg.): The Nature of Creativity. Io. Aufl. Cambridge/New York/Melbourne: Cambridge University Press, 33I3-335.

Cummings, Larry (1965): Organizational Climates for Creativity. In: Academy of Management Journal 8 (3), 220-227.

Czarniawska, Barbara (I998): A Narrative Approach to Organization Studies. London/Thousand Oaks: SAGE University Paper.

Czarniawska, Barbara (2007a): Shadowing and other Techniques for Doing Fieldwork in Modern Societies. Malmö: Liber AB.

Czarniawska, Barbara (2007b): The Styles and the Stylists of Organization Theory. 2. Aufl. In: Christian Knudsen/Haridimos Tsoukas (Hg.): The Oxford Handbook of Organization Theory. New York: Oxford University Press, 237-26r.

Czarniawska, Barbara (2008): Alterity/Identity Interplay in Image Construction. In: Daved Barry/Hans Hansen (Hg.): The SAGE Handbook of New Approaches to Organization Studies. London/Thousand Oaks: SAGE Publications, 49-67.

Czarniawska, Barbara (20I4a): Social Science Research: From Field to Desk. London/Thousand Oaks: SAGE Publications.

Czarniawska, Barbara (20I4b): Storytelling: A Managerial Tool and its Local Translations. In: Gili S. Drori/Markus A. Höllerer/Peter Walgenbach (Hg.): Global Themes and Local Variations in Organization and Management: Perspectives on Glocalization. New York: Routledge, 65-78.

Czarniawska, Barbara (20I5): Narrative, Diskurse und Organisationsforschung. 2. Aufl. In: Rainer Diaz-Bone/Gertraude Krell (Hg.): Diskurs und Ökonomie. Diskursanalytische Perspektiven auf Märkte und Organisationen. Wiesbaden: Springer VS, 79-IO4. 
Czarniawska, Barbara/Joerges, Bernward (1996): Travels of Ideas. In: Dies./ Guje Sevón: Translating Organizational Change. Berlin/New York: de Gruyter, 13-48.

Czarniawska, Barbara/Sevón, Guje (1996a): Introduction. In: Dies. (Hg.): Translating Organizational Change. Berlin/New York: de Gruyter, I-I2.

Czarniawska, Barbara/Sevón, Guje (I996b): Translating Organizational Change. Berlin/New York: de Gruyter.

Czarniawska-Joerges, Barbara (20II): Narratives of Individual and Organizational Identities. In: Stanley A. Deetz (Hg.): Communication Yearbook I7. Florence (KY): Routledge, 193-22I.

Davies, Andrew/Brady, Tim (2000): Organisational capabilities and learning in complex product systems: towards repeatable solutions. In: Research Policy 29 (7/8), 93I-953.

De Vaan, Mathijs/Stark, David/Vedres, Balazs (20I5): Game Changer: The Topology of Creativity. In: American Journal of Sociology I20 (4), I-5I.

Deephouse, David L. (1999): To Be Different, or to Be the Same? It's a Question (And Theory): Of Strategic Balance. In: Strategic Management Journal 20 (2), I47-166.

DeFillippi, Robert (200I): Introduction: Project-based Learning, Reflective Practices and Learning Outcomes. In: Management Learning 32 (I), 5-IO.

DeFillippi, Robert (20I5): Managing Project-Based Organization in Creative Industries. In: Candace Jones/Mark Lorenzen/Jonathan Sapsed (Hg.): The Oxford Handbook of Creative Industries. Oxford: Oxford University Press, 268-283.

DeFillippi, Robert/Grabher, Gernot/Jones, Candace (2007): Introduction to paradoxes of creativity: Managerial and organizational challenges in the cultural economy. In: Journal of Organizational Behavior 28 (5), 5II-52I.

DeFillippi, Robert J./Arthur, Michael B. (1998): Paradox in Project-Based Enterprise: The Case of Film Making. In: California Management Review 40 (2), I25-I4O.

Denzin, Norman K./Lincoln, Yvonna S. (20II): Introduction: The Discipline and Practice of Qualitative Research. 4. Aufl. In: Dies. (Hg.): SAGE Handbook of Qualitative Reserach. London/Thousand Oaks: SAGE Publications, $\mathrm{I}-20$.

Department for Culture, Media and Sport (DCMS; 200I): Creative Industries Mapping Document. London: UK Labour Force, Department for Culture, Media and Sport.

DiMaggio, Paul J. (I99I): Constructing an Organizational Field as a Professional Project: U.S. Art Museums, I920-1940. In: Walter W. Powell/Ders. (Hg.): The New Institutionalism in Organizational Analysis. Chicago/London: The University of Chicago Press, 267-292. 
DiMaggio, Paul J./Powell, Walter W. (1983): The Iron Cage Revisited: Institutional Isomorphism and Collective Rationality in Organizational Fields. In: American Sociological Review 48 (2), I47-160.

Djelic, Marie-Laure/Ainamo, Antti (2005): The Telecom Industry as Cultural Industry? The Transportation of Fashion Logics into the Field of Mobile Telephony. In: Candace Jones/Patricia H. Thornton (Hg.): Transformation in Cultural Industries. Oxford: Elsevier Ltd., 45-80.

Djelic, Marie-Laure/Ainamo, Antti (I999): The Coevolution of New Organizational Forms in the Fashion Industry: A Historical and Comparative Study of France, Italy, and the United States. In: Organization Science Io (5), 622-637.

Dokko, Gina/Rosenkopf, Lori (20I0): Social Capital for Hire? Mobility of Technical Professionals and Firm Influence in Wireless Standards Committees. In: Organization Science 2I (3), 677-695.

Dosi G. (I982): Technological paradigms and technological trajectories. In: Research Policy II, I47-I62.

Dowd, Timothy (2004): The embeddedness of cultural industries. In: Poetics $32, \mathrm{I}-3$.

Drazin, Robert/Schoonhoven, Claudia Bird (I996): Community, Population, and Organization Effects on Innovation: A Multilevel Perspective. In: Academy of Management Journal 39 (5), 1065-1083.

Drazin, Robert/Glynn, Mary Ann/Kazanjian, Robert K. (I999): Multilevel Theorizing About Creativity in Organizations: A Sensemaking Perspective. In: The Academy of Management Review 24 (2), 286-308.

Dutton, Jane E./Dukerich, Janet M. (I99I): Keeping an Eye on the Mirror: Image and Identity in Organizational Adaptation. In: Academy of Management Journal 34 (3), 5I7-554.

Eberle, Thomas S./Maeder, Christoph (2016): Organizational Ethnography. In: David Silverman (Hg.): Qualitative Research. Theory, Method and Practice. 4. Aufl. London/Thousand Oaks: SAGE Publications, I2I-I36.

Elsbach, Kimberly D./Kramer, Roderick M. (2003): Assessing Creativity in Hollywood Pitch Meetings: Evidence for a Dual-Process Model of Creativity Judgments. In: The Academy of Management Journal 46 (3), 283-30I.

Elsbach, Kimberly D./Sutton, Robert I. (I992): Acquiring Organizational Legitimacy through Illegitimate Actions: A Marriage of Institutional and Impression Management. In: The Academy of Management Journal 35 (4), $699-738$.

Emerson, Robert M./Fretz, Rachel I./Shaw, Linda L. (20II): Writing Ethnographic Fieldnotes. 2. Aufl. Chicago/London: The University of Chicago Press. Engwall, Mats (2003): No project is an island: Linking projects to history and context. In: Research Policy 32 (5), 789-808. 
Entwistle, Joanne (2006): The Cultural Economy of Fashion Buying. In: Current Sociology 54 (5), 704-724.

Entwistle, Joanne (2002): The Aesthetic Economy. In: Journal of Consumer Culture 2 (3), 3I7-339.

Entwistle, Joanne/Rocamora, Agnès (2006): The Field of Fashion Materialized: A Study of London Fashion Week. In: Sociology 40 (4), 735-751.

Esposito, Elena (20II): Originality through Imitation: The Rationality of Fashion. In: Organization Studies 32 (5), 603-613.

Feldman, Martha S. (2000): Organizational Routines as a Source of Continuous Change. In: Organization Science II (6), 6II-629.

Feldman, Martha S./Pentland, Brian T. (2003): Reconceptualizing Organizational Routines as a Source of Flexibility and Change. In: Administrative Science Quarterly 48, 94-II8.

Fine, Gary Alan (I996): Justifying Work: Occupational Rhetorics as Resources in Restaurant Kitchens. In: Administrative Science Quarterly 4I (I), 9O-II5.

Fiol, C. Marlene (2005): Intraorganizational Cognition and Interpretation. In: Joel A. C. Baum (Hg.): The Blackwell Companion to Organizations. Oxford: Blackwell Business, II9-I37.

Flick, Uwe (20II): Triangulation. Eine Einführung. 3., aktual. Aufl. Wiesbaden: Springer VS.

Fligstein, Neil/McAdam, Doug (20II): Toward a General Theory of Strategic Action Fields. In: Sociological Theory 29 (I), I-26.

Florida, Richard (2006): The rise of the creative class. II. Aufl. New York: Basic Books.

Flyvbjerg, Bent (2006): Five Misunderstandings About Case-Study Research. In: Qualitative Inquiry I2 (2), 219-245.

Ford, Cameron M. (I996): A Theory of Individual Creative Action in Multiple Social Domains. In: Academy of Management Review 2I (4), III2-II42.

Fortwengel, Johann/Schüssler, Elke/Sydow, Jörg (20I6): Studying Organizational Creativity as Process: Fluidity or Duality? In: Creativity and Innovation Management 2 (2), I-I2.

Foster, Pacey C./Ocejo, Richard E. (2015): Brokerage, Mediation, and Social Networks in the Creative Industries. In: Candace Jones/Mark Lorenzen/Jonathan Sapsed (Hg.): The Oxford Handbook of Creative Industries. Oxford: Oxford University Press, 403-420.

Froschauer, Ulrike (2009): Artefaktanalyse. In: Stefan Kühl/Petra Strodtholz/ Andreas Taffertshofer (Hg.): Handbuch Methoden der Organisationsforschung. Quantitative und Qualitative Methoden. Wiesbaden: VS Verlag für Sozialwissenschaften, 326-347.

Funke, Joachim (2000): Psychologie der Kreativität. In: Rainer M. Holm-Hadulla (Hg.): Kreativität. Heidelberg: Springer, 283-300. Online unter www. 
psychologie.uni-heidelberg.de/ae/allg/mitarb/jf/Funke_20oo_Kreativitaet.pdf.

Garud, Raghu/Simpson, Barbara/Langley, Ann/Tsoukas, Haridimos (2016a): Introduction: How Does Novelty Emerge? In: Dies. (Hg.): The Emergence of Novelty in Organizations. Oxford: Oxford University Press, I-26.

Garud, Raghu/Simpson, Barbara/Langley, Ann/Tsoukas, Haridimos (Hg.; 2or6b): The Emergence of Novelty in Organizations. Oxford: Oxford University Press.

Garud, Raghu/Tuertscher, Philipp/Van de Ven, Andrew H. (2013): Perspectives on Innovation Processes. In: The Academy of Management Annals 7 (I), 775-8i9.

Giertz-Mårtenson, Ingrid (2008): Looking into the Future: A Study of Fashion Forecasting. MA-Thesis: Stockholm University 2006, I-IO.

Gilson, Lucy L. (20I5): Creativity in Teams: Processes and Outcomes in Creative Industries. In: Candace Jones/Mark Lorenzen/Jonathan Sapsed (Hg.): The Oxford Handbook of Creative Industries. Oxford: Oxford University Press, $50-74$.

Gilson, Lucy L./Madjar, Nora (20II): Radical and incremental creativity: Antecedents and processes. In: Psychology of Aesthetics, Creativity, and the Arts 5 (I), $2 \mathrm{I}-28$.

Gilson, Lucy L./Mathieu, John E./Shalley, Christina E./Ruddy, Thomas E. (2005): Creativity and Standardization: Complementary or Conflicting Drivers of Team Effectiveness? In: Academy of Management Journal 48 (3), $52 \mathrm{I}-53 \mathrm{I}$.

Godart, Frédéric C./Maddux, William W./Shipilov, Andrew V./Galinsky, Adam

D. (20I5): Fashion with a Foreign Flair: Professional Experiences Abroad Facilitate the Creative Innovations of Organizations. In: Academy of Management Journal 58 (I), I95-220.

Godart, Frédéric C./Mears, Ashley (2009): How Do Cultural Producers Make Creative Decisions? Lessons from the Catwalk. In: Social Forces 88, 67I692.

Godart, Frédéric C./Shipilov, Andrew V./Claes, Kim (20I4): Making the Most of the Revolving Door: The Impact of Outward Personnel Mobility Networks on Organizational Creativity. In: Organization Science 25, 377-400. Goncalo, Jack A./Duguid, Michelle M. (20I2): Follow the crowd in a new direction: When conformity pressure facilitates group creativity (and when it does not). In: Organizational Behavior and Human Decision Processes II8 (I), I4-23.

Gong, Yaping/Kim, Tae-Yeol/Lee, Deog-Ro (2013): A multilevel Model of Team Goal Orientation, Information Exchange and Creativity. In: Academy of Management Journal 56 (3), 827-851. 
Grabher, Gernot (200I): Ecologies of creativity: The village, the group, and the heterarchic organisation of the British advertising industry. In: Environment and Planning A 33 (2), 35I-374.

Grabher, Gernot (2002): Cool Projects, Boring Institutions: Temporary Collaboration in Social Context. In: Regional Studies 36 (3): 205-2I4.

Grabher, Gernot (2004a): Learning in Projects, Remembering in Networks? Communality, Sociality, and Connectivity in Project Ecologies. In: European Urban and Regional Studies II (2), IO3-I23.

Grabher, Gernot (2004b): Temporary Architectures of Learning: Knowledge Governance in Project Ecologies. In: Organization Studies 25 (9), I49II5I4.

Granovetter, Mark S. (1973): The Strength of Weak Ties. In: American Journal of Sociology 78 (6), I360-I380.

Guilford, Joy Paul (1950): Creativity. In: The American Psychologist 33 (4), 444-454.

Hargadon, Andrew B./Bechky, Beth A. (2006): When Collections of Creatives Become Creative Collectives: A Field Study of Problem Solving at Work. In: Organization Science I7 (4), 484-500.

Hargadon, Andrew B./Douglas, Yellowlees (200I): When Innovations Meet Institutions: Edison and the Design of the Electric Light. In: Administrative Science Quarterly 46 (3), 476-50I.

Hargadon, Andrew/Sutton, Robert (1997): Technology Brokering and Innovation in a Product Development Firm. In: Administrative Science Quarterly 42 (4), 716-749.

Hartley, John/Potts, Jason/Cunningham, Stuart/Flew, Terry/Keane, Michael/ Banks, John (2013): Key Concepts in Creative Industries. London/Thousand Oaks: SAGE Publications.

Harvey, Sarah/Kou, Chia-Yu (20I3): Collective Engagement in Creative Tasks: The Role of Evaluation in the Creative Process in Groups. In: Administrative Science Quarterly 58 (3), 346-386.

Hasse, Raimund/Krücken, Georg (20I4): Decoupling and Coupling in Education. In: Boris Holzer/Fatima Kastner/Tobias Werron (Hg.): From Globalization to World Society. Neo-Institutional and Systems-Theoretical Perspectives. New York: Routledge, I97-2I4.

Hasse, Raimund/Krücken, Georg (2009): Neo-institutionalistische Theorie. In: Georg Kneer/Markus Schroer (Hg.): Handbuch Soziologische Theorien. Wiesbaden: Springer VS, 237-25I.

Hasse, Raimund/Krücken, Georg (2008): Institution. In: Nina Baur u. a. (Hg.): Handbuch Soziologie. Wiesbaden: Springer VS, $\mathrm{I}_{3}-\mathrm{I} 82$.

Hasse, Raimund/Mützel, Sophie/Nyfeler, Judith (2019): Hauptsache neu? Die Organisation von Innovation und Kreativität. In: Maja Apelt u. a. (Hg.): 
Handbuch Organisationssoziologie. Wiesbaden: Springer Reference (im Erscheinen).

Hasse, Raimund/Passarge, Eva (2015): Silicon Valley und sonst nichts Neues? Biotechnologie in der Schweiz als Beispiel für neue Organisationsformen und deren Legitimierung. In: Zeitschrift für Soziologie 44 (I), 6-2I.

Hasse, Raimund/Schmidt, Lucia (2008): Unternehmertum, Arbeit, Sprache. In: Sociologia Internationalis 48 (I), I-28.

Henderson, Rebecca M./Clark, Kim B. (I990): Architectural Innovation: The Reconfiguration of Existing Product Technologies and the Failure of Established Firms. In: Administrative Science Quarterly 35 (I), 9-30.

Hirsch, Paul M. (I972): Processing Fads and Fashions: An Organization-Set Analysis of Cultural Industry Systems. In: American Journal of Sociology $77(4), 639-659$.

Hirsch, Paul M. (2000): Cultural Industries Revisited. In: Organization Science is (3), 356-36r.

Hobday, Mike (2000): The Project-Based Organisation: An Ideal Form for Managing Complex Products and Systems? In: Research Policy 29 (7/8), $87 \mathrm{I}-893$.

Hodgons, Geoffrey M. (2008): The Concept of a Routine. In: Markus C. Becker (Hg.): Handbook of Organizational Routines. Cheltenham (UK)/Northampton (MA): Edward Elgar, I5-28.

Hollingsworth, J. Rogers/Boyer, Robert (I997): Coordination of Economic Actors and Social Systems of Production. In: Dies. (Hg.): Contemporary Capitalism: The Embeddedness of Institutions. Cambridge/New York/Melbourne: Cambridge University Press, I-47.

Hughes, Everett C. (I993): The Sociological Eye: Selected Papers. New Brunswick (NJ): Transaction Publishers.

Hutchins, Edwin (I99I): Organizing Work by Adaptation. In: Organization Science 2 (I), I4-39.

Hutter, Michael (20II): Infinite Surprises: On the Stabilization of Value in the Creative Industries. In: Jens Beckert/Patrik Aspers (Hg.): The Worth of Goods: Valuation and Pricing in the Economy. Oxford: Oxford University Press, 2OI-220.

Jia, Liangding/Shaw, Jason D./Tsui, Anne S./Park, Tae-Youn (20I3): A Social-Structural Perspective on Employee-Organization Relationships and Team Creativity. In: Academy of Management Journal 57 (3), 869-891.

Jones, Candace/Anand, N./Alvarez, Josè Luis (2005): Manufactured Authenticity and Creative Voice in Cultural Industries. In: Journal of Management Studies 42 (5), 893-899.

Jones, Candace/Lichtenstein, Benyamin B. (2009): Temporary Inter-organizational Projects: How Temporal and Social Embeddedness Enhance Coordination and Manage Uncertainty. In: Steve Cropper u. a. (Hg.): The Oxford 
Handbook of Inter-Organizational Relations. Oxford: Oxford University Press, 232-255.

Jones, Candace/Livne-Tarandach, Reut (2008): Designing a Frame: Rhetorical Strategies of Architects. In: Journal of Organizational Behavior 29, 1075Io99.

Jones, Candace/Lorenzen, Mark/Sapsed, Jonathan (20I5): Creative Industries - A Typology of Change. In: Dies. (Hg.): The Oxford Handbook of Creative Industries. Oxford: Oxford University Press, 3-30.

Jones, Candace/Svejenova, Silviya/Strandgaard Pedersen, Jesper (20I6): Misfits, Mavericks and Mainstreams: Drivers of Innovation in Creative Industries. In: Organization Studies 37 (6), 751-768.

Jones, Candace/Thornton, Patricia H. (2005): Transformation in Cultural Industries. Oxford: Elsevier Ltd.

Kadushin, Charles (I976): Networks and Circles in the Production of Culture. In: American Behavioral Scientist is (6), 769-784.

Katz, Ralph (I982): The Effects of Group Longevity on Project Communication and Performance. In: Administrative Science Quarterly 27, 8I-IO4.

Kaufman, James C./Sternberg, Robert J. (2015): The Creative Mind. In: Candace Jones/Mark Lorenzen/Jonathan Sapsed (Hg.): The Oxford Handbook of Creative Industries. Oxford: Oxford University Press, 33-49.

Kawamura, Yuniya (2005): Fashion-ology reprinted. An introduction to fashion studies. London/New York: Bloomsbury Academic.

Kern, Anja (2006): Exploring the Relation between Creativity and Rules. The Case of the Performing Arts. In: International Studies of Management \& Organization 36 (I), 63-80.

Khaire, Mukti (20I5): Entrepreneurship in Creative Industries and Cultural Change. In: Candace Jones/Mark Lorenzen/Jonathan Sapsed (Hg.): The Oxford Handbook of Creative Industries. Oxford: Oxford University Press, 200-2I8.

Khaire, Mukti (20I4): Fashioning an Industry: Socio-cognitive Processes in the Construction of Worth of a New Industry. In: Organization Studies 35 (I), 4I-74.

Khaire, Mukti/Hall, Erika V. (20I6): Medium and Message: Globalization and innovation in the production field of Indian fashion. In: Organization Studies $37(6), 845-865$.

Kieser, Alfred (20I4): Der Situative Ansatz. In: Ders./Mark Ebers (Hg.): Organisationstheorien. 7. Aufl. Stuttgart: Kohlhammer, I64-194.

Kieser, Alfred/Walgenbach, Peter (20I0): Organisation. 6. Aufl. Stuttgart: Schäffer-Poeschel.

Koch, Jochen/Wenzel, Matthias/Senf, Ninja Natalie/Maibier, Corinna (20I8): Organizational Creativity as an Attributional Process: The Case of Haute Cuisine. In: Organization Studies 39 (2/3), 25I-270. 
Krämer, Hannes (20I4): Die Praxis der Kreativität. Eine Ethnografie kreativer Arbeit. Bielefeld: transcript.

Krause-Jensen, Jakob (2013): Looking into the box: design and innovation at Bang \& Olufsen. In: Brian Moeran (Hg.): Exploring Creativity. Evaluative Practices in Innovation, Design, and the Arts. Cambridge/New York/Melbourne: Cambridge University Press, I46-I7I.

Kruse, Jan (20I5): Qualitative Interviewforschung. 2. Aufl. Weinheim/Basel: Beltz Juventa.

Kühl, Alicia (20I5): Modenschauen. Die Behauptung des Neuen in der Mode. Bielefeld: transcript.

Kuhlmann, Martin (2005): Beobachtungsinterview. In: Stefan Kühl/Petra Strodtholz/Andreas Taffertshofer (Hg.): Handbuch Methoden der Organisationsforschung. Quantitative und Qualitative Methoden. Wiesbaden: VS Verlag für Sozialwissenschaften, 78-ıoo.

Kurtzberg, Terri R./Amabile, Teresa M. (2010): From Guilford to Creative Synergy: Opening the Black Box of Team-Level Creativity. In: Creativity Research Journal i3 (3), 285-294.

Lampel, Joseph (2008): The Genius Behind the System: The Emergence of the Central Producer System in the Hollywood Motion Picture Industry. In: Ders/Jamal Shamsie/ Theresa K. Lant (Hg.): The Business of Culture. Strategic Perspectives on Entertainment and Media. 2. Aufl. New York/London: Psychology Press, 4I-56.

Lampel, Joseph/Lant, Theresa K./Shamsie, Jamal (2000): Balancing Act: Learning from Organizing Practices in Cultural Industries. In: Organization Science II (3), 263-269.

Lampel, Joseph/Shamsie, Jamal/ Lant, Theresa K (2008a): The Business of Culture. Strategic Perspectives on Entertainment and Media. 2. Aufl. New York/London: Psychology Press.

Lampel, Joseph/Shamsie, Jamal/ Lant, Theresa K. (2008b): Toward a Deeper Understanding of Cultural Industries. In: Dies. (Hg.): The Business of Culture. Strategic Perspectives on Entertainment and Media. 2. Aufl. New York/London: Psychology Press, 3-I4.

Lampel, Joseph/Shamsie, Jamal/Lant, Theresa K. (2008c): Untangling the Complexities of Cultural Industries: Directions for Future Research. In: Dies. (Hg.): The Business of Culture. Strategic Perspectives on Entertainment and Media. 2. Aufl. New York/London: Psychology Press, 289-304. Landry, Charles (2004): The Creative City: A Toolkit for Urban Innovators. 4. Aufl. London/Sterling (VA): Earthscan Publications.

Lant, Theresa K. (2005): Organizational Cognition and Interpretation. In: Joel A. C. Baum (Hg.): The Blackwell Companion to Organizations. Oxford: Blackwell Business, 334-362. 
Lant, Theresa K./Baum, Joel A. C. (I995): Cognitive Sources of Socially Constructed Competitive Groups. Examples from the Manhattan Hotel Industry. In: Richard W. Scott/Soren Christensen (Hg.): The Institutional Construction of Organizations. London/Thousand Oaks: SAGE Publications, I5-38.

Lant, Theresa K./Hewlin, Patricia F. (2008): Silicon Alley.com: Struggling for Legitimacy in New Media. In: Joseph Lampel/Jamal Shamsie/Theresa K. Lant (Hg.): The Business of Culture. Strategic Perspectives on Entertainment and Media. 2. Aufl. New York/London: Psychology Press, 227-238.

Lee, You-Na/Walsh, John P./Wang, Jian (2015): Creativity in scientific teams: Unpacking novelty and impact. In: Research Policy 44 (3), 684-697.

Liebold, Renate/Trinczek, Rainer (2009): Experteninterview. In: Stefan Kühl/ Petra Strodtholz/Andreas Taffertshofer (Hg.): Handbuch Methoden der Organisationsforschung. Quantitative und Qualitative Methoden. Wiesbaden: VS Verlag für Sozialwissenschaften, 32-56.

Lindkvist, Lars (2005): Knowledge Communities and Knowledege Collectivities: A Typology of Knowledge Work in Groups. In: The Journal of Management Studies 42 (6), II89-г210.

Lipovetsky, Gilles (2002): The Empire of Fashion. Dressing Modern Democracy. 3. Aufl. Princeton (NJ): Princeton University Press.

Lofland, John (I97I): Feld-Notizen (Notes). In: Analyzing Social Settings, IIoI2O.

Lounsbury, Michael/Glynn, Mary Ann (200I): Cultural entrepreneurship: Stories, legitimacy, and the acquisition of resources. In: Strategic Management Journal 22 (6/7), 545-564.

Lundin, Rolf A./Söderholm, Anders (I995): A theory of the temporary organization. In: Scandinavian Journal of Management II (4), 437-455.

Madjar, Nora/Oldham, Greg R./Pratt, Michael G. (2002): There's No Place Like Home? The Contributions of Work and Nonwork Creativity Support to Employees' Creative Performance. In: Academy of Management Journal 45 (4), $757-767$.

Maeder, Christoph (20II): Ethnografische Semantik. In: Ralf Bohnsack/Winfried Marotzki/Michael Meuser (Hg.): Hauptbegriffe Qualitativer Sozialforschung. 3., durchges. Aufl. Opladen/Farmington Hills (MI): Barbara Budrich, $52 \mathrm{f}$.

Mangematin, Vincent/Sapsed, Jonathan/Schüssler, Elke (20I4): Disassembly and reassembly: An introduction to the Special Issue on digital technology and creative industries. In: Technological Forecasting and Social Change 83 (I), I-9.

March, James G. (I991): Exploration and Exploitation in Organizational Learning. In: Organization Science 2 (I), 7I-87.

March, James G./Olsen, Johan P. (I989): Rediscovering Institutions: The organizational Basis of Politics. New York: The Free Press. 
March, James G./Simon, Herbert A. (1958): Organizations. New York: Wiley. Mareis, Claudia (20I6a): Angewandte Imagination und Kreativität um I960. Eine Einführung. In: Dies. (Hg.): Designing Thinking. Angewandte Imagination und Kreativität um i960. Paderborn: Wilhelm Fink, 9-37.

Mareis, Claudia (Hg.; 20I6b): Designing Thinking. Angewandte Imagination und Kreativität um iو6o. Paderborn: Wilhelm Fink.

Martin, Joanne/Feldman, Martha S./Hatch, Mary Jo/Sitkin, Sim B. (1983): The Uniqueness Paradox in Organizational Stories. In: Administrative Science Quarterly 28 (3), 438-453.

McRobbie, Angela (20II): Re-Thinking Creative Economy as Radical Social Enterprise. In: Variant (4I), $32 \mathrm{f}$.

McRobbie, Angela (20I6): Towards a Sociology of Fashion Micro-Enterprises: Methods for Creative Economy Research. In: Sociology 50 (5), 934-948.

Mears, Ashley (20II): Pricing Looks: Circuits of Vlaue in Fashion Modeling Markets. In: Jens Beckert/Patrik Aspers (Hg.): The Worth of Goods: Valuation and Pricing in the Economy. Oxford: Oxford University Press, I55-I77.

Meyer, Christian (2013): Finding the Right Place: On the Rhetorics of Field Access. In: Ivo A. Strecker/Shauna LaTosky (Hg.): Writing in the Field: Festschrift for Steven Tyler. Münster: LIT Verlag, 2I-32.

Meyer, John W./Rowan, Brian (I977): Institutionalized Organizations: Formal Structure as Myth and Ceremony. In: American Journal of Sociology 83 (2), $340-363$.

Meyerson, Debra/Weick, Karl E./Kramer, Roderick M. (2006): Swift Trust and Temporary Groups. In: Roderick M. Kramer (Hg.): Organizational Trust: A Reader. Oxford: Oxford University Press, 4I5-444.

Mintzberg, Henry (Hg.; I989): Mintzberg on Management: Inside our strange world of organizations. New York: The Free Press.

Moeran, Brian (2008): Economic and cultural production as structural paradox: the case of international fashion magazine publishing. In: International Review of Sociology I8 (2), 267-28I.

Moeran, Brian (2009): The organization of creativity in Japanese advertising production. In: Human Relations 62 (7), 963-985.

Morean, Brian (20I2): More Than Just A Fashion Magazine. In: Ders./Ana Alacovska (Hg.): Creative Industries: Critical Readings. London/Thousand Oaks: SAGE Publications, Bd. 3: Organization, 4I-56.

Moeran, Brian/Christensen, Bo T. (20I3): Introduction. In: Ders. (Hg.): Exploring Creativity. Evaluative Practices in Innovation, Design, and the Arts. Cambridge/New York/Melbourne: Cambridge University Press, I-42.

Mora, Emanuela (2006): Collective production of creativity in the Italian fashion system. In: Poetics 34 (6), 334-353.

Mützel, Sophie (20I5): Geschichten als Signale. Zur diskursiven Konstruktion von Märkten. In: Rainer Diaz-Bone/Gertraude Krell (Hg.): Diskurs und 
Ökonomie. Diskursanalytische Perspektiven auf Märkte und Organisationen. 2. Aufl. Wiesbaden: Springer VS, $263-284$.

Mützel, Sophie (20I7): Markets from stories. Habil. Humboldt-Universität Berlin, Kultur-, Sozial- und Bildungswissenschaftliche Fakultät.

Nelson, Richard R./Winter, Sidney G. (I977): In Search of Useful Theory of Innovation. In: Research Policy 6 (I), 36-76.

Nelson, Richard R./Winter, Sidney G. (1982): An Evolutionary Theory of Economic Change. Cambridge (MA): Harvard University Press.

Nelson, Richard R./Winter, Sidney G. (20I4): Evolutionary Theorizing in Economics. In: The Journal of Economic Perspectives i6 (2), 23-46.

Obstfeld, David (2012): Creative Projects: A Less Routine Approach Toward Getting New Things Done. In: Organization Science 23 (6), I57I-I592.

Oldham, Greg R./Cummings, Anne (1996): Employee Creativity: Personal and Contextual Factors at Work. In: Academy of Management Journal 39 (3), 607-634.

Ortmann, Günther/Sydow, Jörg (20I8): Dancing in chains: Creative practices in/of organizations. In: Organization Studies 39 (7), 899-921.

Orton, J. Douglas/Weick, Karl E. (1990): Loosely Coupled Systems: A Reconceptualization. In: The Academy of Management Review I5 (2), 203-223.

Osborne, Thomas (2003): Against >creativity<: A philistine rant. In: Economy and Society 32 (4), 507-525.

Patriotta, Gerardo (2003): Sensemaking on the shop floor: Narratives of knowledge in organizations. In: Journal of Management Studies 40 (2), 348-375.

Patriotta, Gerardo/Hirsch, Paul M. (20I6): Mainstreaming Innovation in Art Worlds: Cooperative links, conventions and amphibious artists. In: Organization Studies 37 (6), 867-887.

Patriotta, Gerardo/Lanzara, Giovan Francesco (2006): Identity, Institutions, and New Work Roles. The Case of a Green Field Automotive Factory. In: American Behavioral Scientist 49 (7), 987-999.

Pedersen, Birgit Lyngbye (20I2): Dressed up for the International Market: A Study of the Danish Fashion Fair Promoting the Danis Fashion Industry, I947-I970. In: Peter McNeil/Louise Wallenberg (Hg.): Nordic Fashion Studies. Stockholm: AXL Books, II5-I30.

Pentland, Brian T. (I995): Grammatical Models of Organizational Processes. In: Organization Science 6 (5), 54I-556.

Pentland, Brian T. (1999): Building process theory with narrative: From description to explanation. In: Academy of Management Review 24 (4), 7II724 .

Pentland, Brian T./Rueter, Henry H. (I994): Organizational Routines as Grammars of Action. In: Administrative Science Quarterly 39 (3), 484-510. 
Perretti, Fabrizio/Negro, Giacomo (2007): Mixing genres and matching people: A study in innovation and team composition in Hollywood. In: Journal of Organizational Behavior 28, 563-586.

Perry-Smith, Jill E. (2006): Social Yet Creative: The Role of Social Relationships in Facilitating Individual Creativity. In: Academy of Management Journal 49 (I), 85-IOI.

Perry-Smith, Jill E./Mannucci, Pier Vittorio (20I6): From creativity to innovation: The social network drivers of the four phases of the idea journal. In: Academy of Management Review 42 (I), 53-79.

Petkova, Iva (20I6): Between high-tech and high-fashion: How E-Commerce fashion organizations gain moral and pragmatic legitimacy in the fashion field. In: Poetics 57, 55-69.

Polletta, Francesca/Chen, Pang Ching Bobby/Gharrity Gardner, Beth/Motes, Alice (20II): The Sociology of Storytelling. In: Annual Review of Sociology 37 (I), I09-I30.

Porac, Joseph F./Thomas, Howard (1990): Taxonomic Mental Models in Competitor Definition. In: Academy of Management Journal I5 (2), 224-240.

Porac, Joseph F./Thomas, Howard/Baden-Fuller, Charles (I989): Cognitive Communities: The Case of Scottish Knitwear Manufacturers. In: Journal of Management Studies 26 (4), 397-4I6.

Potts, Jason/Cunningham, Stuart (2008): Four models of the creative industries. International Journal of Cultural Policy I4 (3), 233-247.

Potts, Jason/Cunningham, Stuart/Hartley, John/Ormerod, Paul (2008): Social network markets: A new definition of the creative industries. In: Journal of Cultural Economics 32 (3), I67-185.

Powell, Walter W. (I990): Neither Market nor Hierarchy: Network Forms of Organization. In: Research in Organizational Behavior I2, 295-336.

Powell, Walter W./Colyvas, Jeannette A. (2013): Microfoundations of Institutional Theory. In: Royston Greenwood u. a. (Hg.): The SAGE Handbook of Organizational Institutionalism. 2. Aufl. London/Thousand Oaks (CA): SAGE Publications [2008], 276-298.

Powell, Walter W./DiMaggio, Paul J. (199I): The New Institutionalism in Organizational Analysis. Chicago/London: The University of Chicago Press.

Powell, Walter W./Koput, Kenneth W./Smith-Doerr, Laurel (1996): Interorganizational Collaboration and the Locus of Innovation: Networks of Learning in Biotechnology. In: Administrative Science Quarterly 4I (I), II6-I45.

Powell, Walter W./Smith-Doerr, Laurel (2003): Networks and Economic Life. In: Neil J. Smelser/Richard Swedberg (Hg.): The Handbook of Economic Sociology. 2. Aufl. Princeton (NJ): Princeton University Press, I-7I.

Powell, Walter W./Smith-Doerr, Laurel (I994): Networks of Economic Life. In: Neil J. Smelser/Richard Swedberg (Hg.): The Handbook of Economic Sociology. Princeton (NJ): Princeton University Press, 368-402. 
Pratt, Andy C./Eikhof, Doris Ruth/Sawyer, Keith/Austin, Robert D./Johnson, Peter/Straw, Martyn/Wikstrom, Patrik/Ren, Jie/Lau, Filip (2015): Opinions: All about Creativity and Innovation. In: Journal of Business Anthropology 4 (2), 228-297.

Raunig, Gerald/Wuggenig, Ulf (20I6): Kritik der Kreativität. Wien: Transversal Texts.

Raustiala, Kal/Sprigman, Christopher (2006): The piracy paradox: Innovation and intellectual property in fashion design. In: Virginia Law Review 92 (8), I687-I777.

Reckwitz, Andreas (20I4): Die Erfindung der Kreativität. 4. Aufl. Berlin: Suhrkamp.

Reckwitz, Andreas (20I6): Kreativität und Soziale Praxis. Studien zur Sozialund Gesellschaftstheorie. Bielefeld: transcript.

Rhodes, Carl/Brown, Andrew D. (2005): Narrative, organizations and research. In: International Journal of Management Reviews 7 (3), I67-188.

Richards, Lyn (Hg.; 20I5): Handling Qualitative Data: A Practical Guide. 3. Aufl. London/Thousand Oaks: SAGE Publications.

Rogers, Everett M. (2003): Diffusion of Innovations. 5. Aufl. New York: Free Press.

Rosso, Brent D. (20I4): Creativity and Constraints: Exploring the Role of Constraints in the Creative Processes of Research and Development Teams. In: Organization Studies 35 (4), 55 I-585.

Runco, Mark A. (2007): Creativity. Theories and Themes: Research, Development, and Practice. Burlington/San Diego: Elsevier.

Sacramento, Claudia A./Dawson, Jeremy F./West, Michael A. (2008); Team Creativity: More Than the Sum of Its Parts? In: Michael D. Mumford/Samuel T. Hunter/Katrina E. Bedell-Avers (Hg.): Multi-Level Issues in Creativity and Innovation. Bingley (UK): Emerald Group, 269-287.

Sahlin-Andersson, Kerstin (1996): Imitating by Editing Success: The Construction of Organizational Fields. In: Barbara Czarniawska/Guje Sevón: Translating Organizational Change. Berlin/New York: de Gruyter, 69-92.

Sahlin-Andersson, Kerstin/Linderoth, Henrik (2002): Bridging the gap between temporality and permanency: Action and the temporary organization. In: Dies./Anders Söderholm (Hg.): Beyond project management: New perspectives on the temporary-permanent dilemma. Malmö: Liber AB, 224-240.

Sahlin, Kerstin/Wedlin, Linda (2013): Circulating Ideas: Imitation, Translation and Editing. In: Royston Greenwood u. a. (Hg.): The SAGE Handbook of Organizational Institutionalism. 2. Aufl. London/Thousand Oaks (CA): SAGE Publications [2008], 218-242.

Saunders, Carol S./Ahuja, Manju K. (2006): Are all distributed teams the same? In: Small Group Research 37 (6), 662-700. 
Schneiberg, Marc/Clemens, Elisabeth S. (2006): The Typical Tools for the Job: Research Strategies in Institutional Analysis. In: Sociological Theory 24 (3), 195-227.

Schumacher, Christina/Glaser, Marie Antoinette (2008): Kreativität in der Architekturausbildung. Erkundung zu einem disziplinären Mythos. In: Birgit Althans u. a. (Hg.): Kreativität. Eine Rückrufaktion. Bielefeld: transcript (Zeitschrift für Kulturwissenschaften I), I3-30.

Schwab, Andreas/Miner, Anne S. (2008): Learning in hybrid project systems: The effects of project performance on repeated collaboration. In: Academy of Management Journal 5I (6), III7-II49.

Scott, W. Richard (I994): Conceptualizing Organizational Fields. Linking Organizations and Societal Systems. In: Hans-Ulrich Derlien/Uta Gerhardt/ Fritz W. Scharpf (Hg.): Systemrationalität und Partialinteresse. Festschrift für Ruth Mayntz. Baden-Baden: Nomos, 203-22I.

Scott, W. Richard (2008): Observations on Research on Cultural Industries. In: Joseph Lampel/Jamal Shamsie/Theresa K. Lant (Hg.): The Business of Culture. Strategic Perspectives on Entertainment and Media. 2. Aufl. New York/London: Psychology Press, I5-22.

Scott, W. Richard/Meyer, John W. (I99I): The Organization of Societal Sectors: Propositions and Early Evidence. In: Walter W. Powell/Paul J. DiMaggio (Hg.): The New Institutionalism in Organizational Analysis. Chicago/London: The University of Chicago Press, ro8-140.

Selznick, Philip (I984): Leadership in Administration. Berkley/Los Angeles/ London: University of California Press.

Sevón, Guje (I996): Organizational Imitation in Identity Transformation. In: Barbara Czarniawska/Dies.: Translating Organizational Change. Berlin/ New York: de Gruyter, 49-67.

Shalley, Christina E./Gilson, Lucy L. (20I7): Creativity and the Management of Technology: Balancing Creativity and Standardization. In: Production and Operations Management 26 (4), 605-6r6.

Shalley, Christina E./Gilson, Lucy L./Blum, Terry C. (2000): Matching Creativity Requirements and the Work Environment: Effects on Satisfaction and Intentions to Leave. In: Academy of Management Journal 43 (2), 2I5-223.

Shaw, Gordon/Brown, Robert/Bromiley, Philip (I998): Strategic Stories: How 3M is Rewrinting Business Planning. In: Harvard Business Review 76 (3), $4 \mathrm{I}-50$.

Shiu, Eric (20I4): Creativity Research. London/New York: Routledge.

Silverman, David (20I3): Doing Qualitative Research. 4. Aufl. London/Thousand Oaks: SAGE Publications.

Silverman, David (20I4): Interpreting Qualitative Data. 5. Aufl. London/Thousand Oaks: SAGE Publications. 
Silverman, David (20I7): How was it for you? The Interview Society and the irresistible rise of the (poorly analyzed) interview. In: Qualitative Research I7 (2), I44-I58.

Simmel, Georg (1986): Die Mode. In: Silvia Bovenschen (Hg.): Die Listen der Mode. Frankfurt am Main: Suhrkamp, I79-207.

Simmel, Georg (20I3): Fashion. In: American Journal of Sociology 62 (6), 54I$55^{8}$.

Simonton, Dean Keith (I999): Origins of Genius. Oxford: Oxford University Press.

Simonton, Dean Keith (2000): Creativity: Cognitive, Personal, Developmental, and Social Aspects. In: American Psychologist 55 (I), I5I-I58.

Simonton, Dean Keith (2004): Group artistic creativity: Creative clusters and cinematic success in feature films. In: Journal of Applied Social Psychology 34 (7), I494-I520.

Simonton, Dean Keith (2010): The Psychology of Creativity. A Historical Perspective. In: History (January 2012), 37-4I.

Skov, Lise (2006): The Role of Trade Fairs in the Global Fashion Business. In: Current Sociology 54 (5), 764-783.

Slavich, Barbara/Svejenova, Silviya (2016): Managing Creativity: A Critical Examination, Synthesis, and New Frontiers. In: European Management Review I3 (4), 237-250.

Sohlberg, Peter/Leiulfsrud, Håkon (20I7): Conceptual Constructionism: An introduction. In: Dies. (Hg.): Concepts in Action: Conceptual Constructionism. Leiden: Brill, I-22.

Sole, Deborah/Wilson, Daniel G. (I999): Storytelling in Organizations: The power and traps of using stories to share knowledge in organizations. In: Training and Development 53, I-I2.

Sombart, Werner (I986): Wirtschaft und Mode. In: Silvia Bovenschen (Hg.): Die Listen der Mode. Frankfurt am Main: Suhrkamp, 80-I05.

Söndermann, Michael/Weckerle, Christoph (2008): Zweiter Zürcher Kreativwirtschaftsbericht. Empirisches Portrait der Kreativwirtschaft Zürich. Zürich. Online unter www.suz.uzh.ch/dam/jcr:0০o০o০oo-I5fd-7ade-ffffffffbf503cbd/13.Io.Zweiter_ZHKWBericht.pdf.

Sonenshein, Scott (20I4): How Organizations Foster the Creative Use of Resources. In: Academy of Management Journal 57 (3), 8I4-848.

Sonenshein, Scott (2016): Routines and Creativity: From Dualism to Duality. In: Organization Science 27 (3), 739-758.

Sriraman, Bharath/Harris, Anne (20I4): Creative Turn: Toward a New Aesthetic Imaginary. Rotterdam: Sense Publishers.

Stark, David (20II): What's Valuable? In: Jens Beckert/Patrik Aspers (Hg.): The Worth of Goods: Valuation and Pricing in the Economy. Oxford: Oxford University Press, 319-338. 
Sternberg, Robert J. (2006): The Nature of Creativity. In: Creativity Research Journal I8 (I), 37-4I.

Sternberg, Robert J. (2007): Handbook of Creativity. Io. Aufl. Cambridge/New York/Melbourne: Cambridge University Press.

Stinchcombe, Arthur L. (I959): Bureaucratic and Craft Administration of Production: A Comparative Study. In: Administrative Science Quarterly 4 (2), I68-I87.

Suchman, Mark C. (I995): Managing Legitimacy: Strategic and Institutional Approaches. In: Academy of Management Review 20 (3), 57I-6Io.

Suddaby, Roy/Greenwood, Royston (2005): Rhetorical Strategies of Legitimacy. In: Administrative Science Quarterly 50 (I), 35-67.

Suddaby, Roy/Haugh, Helen/Doherty, Bob/Huybrechts, Benjamin (20I7): Managing the Authenticity-Legitimacy Paradox: The Rhetorical Construction of Fair Trade, I999-20I3 (Seminar am Amsterdam Business Research Institute).

Swap, Walter/Leonard, Dorothy/Shields, Mimi/Abrams, Lisa (200I): Using Mentoring and Storytelling to Transfer Knowledge in the Workshop. In: Journal of Management Information Systems I8 (I), 95-II4.

Swedberg, Richard (2012): Theorizing in sociology and social science: Turning to the context of discovery. In: Theory and Society 4I (I), I-4O.

Sydow, Jörg/Lindkvist, Lars/DeFillippi, Robert (2004): Project-Based Organizations, Embeddedness and Repositories of Knowledge: Editorial. In: Organization Studies 25 (9), I475-I489.

Sydow, Jörg/Staber Udo (2002): The Institutional Embeddedness of Project Networks: The Case of Content Production in German Television. In: Regional Studies 36 (3), 215-227.

Taylor, Alva/Greve, Heinrich R. (2006): Superman or the Fantastic Four? Knowledge Combination and Experience in Innovative Teams. In: Academy of Management Journal 49 (4), 723-740.

Taylor, Melissa (2005): Culture transition: Fashion's cultural dialogue between commerce and art. In: Fashion Theory - Journal of Dress Body and Culture $9(4), 445-460$.

Thompson, James D. (2008): Organizations in Action. 6. Aufl. New Brunswick (NJ): Transaction Publishers.

Titton, Monica (20I2): Andys Erbe: Über das Verhältnis von Mode und Kunst am Beispiel der Künstlerkollaborationen des Luxuslabels Louis Vuitton. In: Sociologia Internationalis 50 (I/2), $\mathrm{I}_{45}-\mathrm{I} 65$.

Torrance, Ellis Paul (I966): Torrance Tests of Creative Thinking. Princeton: Personnel Press.

Tschang, Ted F. (2007): Balancing the Tensions Between Rationalization and Creativity in the Video Games Industry. In: Organization Science I8 (6), 989-1005. 
Tschmuck, Peter (2012): How Creative Are the Creative Industries? A Case of the Music Industry. In: Brian Moeran/Ana Alacovska (Hg.): Creative Industries: Critical Readings. Bd. I: Concepts. London/New York, 268-28I.

Tuschman, Mary J./Benner, Michael L. (2003): Exploitation, Exploration, and Process Management: The Productivity Dilemma Revisited. In: The Academy of Management Review 28 (2), 238-256.

UNESCO (2013): Creative Economy Report 20I3. New York/Paris. Online unter www.unesco.org/culture/pdf/creative-economy-report-2or3.pdf.

United Nations Conference on Trade and Development (UNCTAD; 2008a): Creative Economy Report. Geneva. Online unter https://unctad.org/en/pages/PublicationArchive.aspx? publicationid $=945$.

United Nations Conference on Trade and Development (UNCTAD; 2008b): Creative Economy Report Summary: The challenge of assessing the creative economy towards informed policy-making. Geneva. Online unter https://unctad.org/en/Docs/ditc20082ceroverview_en.pdf

Unsworth, Kerrie L. (200I): Unpacking Creativity. In: The Academy of Management Review 26 (2), 289-297.

Unsworth, Kerrie L./Clegg, Chris W. (2010): Why do employees undertake creative action? In: Journal of Occupational and Organizational Psychology 83, 77-99.

Uzzi, Brian (1996): The sources and consequences of embeddedness for the economic performance of organizations: The network effect. In: American Sociological Review 6r (4), 674-698.

Uzzi, Brian (I997): Social Structure and Competition in Interfirm Networks: The Paradox of Embeddedness. In: Administrative Science Quarterly 42 (I), 35-67.

Uzzi, Brian/Spiro, Jarrett (2005): Collaboration and Creativity: The Small World Phenomenon. In: American Journal of Sociology III (2), 447-504.

Van de Ven, Andrew H./Scott Poole, Marshall (2005): Field Research Methods. In: Joel A. C. Baum (Hg.): The Blackwell Companion to Organizations. Oxford: Blackwell Business, I-I9.

Van den Berg, Kare (2009): Drei Absagen der Kunst an ihren erweiterten Begriff. In: Stephan A. Jansen/Eckhard Schröter/Nico Stehr (Hg.): Rationalität der Kreativität? Multidisziplinäre Beiträge zur Analyse der Produktion, Organisation und Bildung von Kreativität. Wiesbaden: VS Verlag für Sozialwissenschaften, 207-224.

Vangkilde, Kasper T. (2013): In search of a creative concept in HUGO BOSS. In: Brian Moeran (Hg.): Exploring Creativity. Evaluative Practices in Innovation, Design, and the Arts. Cambridge/New York/Melbourne: Cambridge University Press, 69-95.

Veblen, Thorstein (I986): Theorie der feinen Leute. In: Silvia Bovenschen (Hg.): Die Listen der Mode. Frankfurt am Main: Suhrkamp, Io6-I55. 
Ventresca, Marc J./Kaghan, William N. (2008): Routines, >going concerns< and innovation: towards an evolutionary economic sociology. In: Markus C. Becker (Hg.): Handbook of Organizational Routines. Cheltenham (UK)/ Northampton (MA): Edward Elgar, 52-86.

Ventresca, Marc J./Mohr, John W. (2005): Archival Research Methods. In: Joel A. C. Baum (Hg.): The Blackwell Companion to Organizations. Oxford: Blackwell Business, 805-828.

Wallace, Doris B./Gruber, Howard E. (I989): Creative People at Work. New York/Oxford: Oxford University Press.

Watson, Elizabeth (2007): Who or What Creates? A Conceptual Framework for Social Creativity. In: Human Resource Development Review 6 (4), 4I9-44I. Weckerle, Christoph/Page, Roman/Grand, Simon (20I6): Von der Kreativwirtschaft zu den Creative Economies. Zürich. Online unter www.creativeeconomies.com/downloads/creative-economy-report-2oi6.pdf.

Weckerle, Christoph/Söndermann, Michael (2005): Kreativwirtschaft Zürich. Studie I : Der privatwirtschaftliche Teil des kulturellen Sektors im Kanton Zürich. Zürich. Online unter https://awa.zh.ch/internet/volkswirtschaftsdirektion/awa/de/standortfoerderung/cluster/kreativwirtschaft/_jcr_content/contentPar/downloadlist/downloaditems/70_I384509183891.spooler. download.1384509075385.pdf/Erster_Kreativwirtschaftsbericht_Teil_I.pdf

Weckerle, Christoph/Söndermann, Michael/Hofecker, Franz-Otto (2003): Kultur.Wirtschaft.Schweiz. Das Umsatz- und Beschäftigungspotential des Kulturellen Sektors: Erster Kulturwirtschaftsbericht Schweiz. Zürich.

Weckerle, Christoph/Theler, Hubert (20I0): Dritter Kreativwirtschaftsbericht Zürich: Die Bedeutung der Kultur- und Kreativwirtschaft für den Standort Zürich. Zürich. Online unter https://awa.zh.ch/internet/volkswirtschaftsdirektion/awa/de/standortfoerderung/cluster/kreativwirtschaft/_jcr_content/contentPar/downloadlist/downloaditems/73_I384509200092.spooler.download.r384509056045.pdf/Dritter_Kreativwirtschaftsbericht.pdf

Weick, Karl E. (I995): Sensemaking in Organizations. London/Thousand Oaks (CA): SAGE Publications.

Weick, Karl E./Sutcliffe, Kathleen M./Obstfeld, David (2005): Organizing the Process of Sensemaking. In: Organization Science I6 (4), 409-42I.

Weisberg, Robert W. (2006): Creativity. Hoboken (NJ): John Wiley \& Sons.

Werven, Ruben van/Bouwmeester, Onno/Cornelissen, Joep P. (2015): The power of arguments: How entrepreneurs convince stakeholders of the legitimate distinctiveness of their ventures. In: Journal of Business Venturing 30 (4), 6i6-63i.

Westenholz, Ann (2006): Identity Work and Meaning Arena - Beyond Actor/ Strucutre and Micro/Macro Distinctions in an Empirical Analysis of IT Workers. In: American Behavioral Scientist 49 (7), IOI5-IO29. 
Woodman, Richard W./Sawyer, John E./Griffin, Ricky W. (I993): Toward a Theory of Organizational Creativity. In: Academy of Management Review I8 (2), 293-32I.

Wooten, Melissa/Hoffman Andrew J. (2013): Organizational Fields: Past, Present and Future. In: Royston Greenwood u. a. (Hg.): The SAGE Handbook of Organizational Institutionalism. 2. Aufl. London/Thousand Oaks (CA): SAGE Publications [2008], I30-I47.

Wuggenig, Ulf (20I6): Kreativitätsbegriffe. Von der Kritik zu Assimilation, Vergiftung, Ausschlag. Vorwort zur Neuauflage von Kritik der Kreativität. In: Gerald Raunig/Ders. (Hg.): Kritik der Kreativität. Wien: Transversal Texts, II-77.

Ziege, Eva-Maria (20II): Die Kunst der Unterscheidung. Soziologie der Mode. In: Leviathan 39 (I), I4I-I59.

Zilber, Tammar B. (2009): Institutional maintenance as narrative acts. In: Thomas B. Lawrence/Roy Suddaby/Bernhard Leca (Hg.): Institutional Work: Actors and Agency in institutional Studies of Organizations. Cambridge/ New York/Melbourne: Cambridge University Press, 205-235.

Zilber, Tammar B. (2002): Institutionalization as an Interplay Between Actions, Meanings, and Actors: The Case of a Rape Crisis Center in Israel. In: The Academy of Management Journal 45 (I), 234-254. 


\section{Anhänge}

\section{Anhang A: Übersicht Interviews UND BEOBACHTUNGSPROTOKOLLE}

\begin{tabular}{|l|l|l|}
\hline Datum & Standort & Anlass \\
\hline 30.I0.I4 & Standort I & $\begin{array}{l}\text { Interview mit } \\
\text { Designerin }\end{array}$ \\
\hline I5.I2.I4 & Standort 2 & Beobachtungsprotokoll: Fotoshooting \\
\hline 26.0I.I5 & Standort I & $\begin{array}{l}\text { Interview mit PR- } \\
\text { Manager }\end{array}$ \\
\hline 26.0I.I5 & Standort I & $\begin{array}{l}\text { Interview HR- } \\
\text { Managerin/Ladenchefin }\end{array}$ \\
\hline 27.0I.I5 & Standort I & $\begin{array}{l}\text { Interview mit Art } \\
\text { Director/CEO }\end{array}$ \\
\hline 28.0I.I5 & Standort I & $\begin{array}{l}\text { Interview mit } \\
\text { Designerin }\end{array}$ \\
\hline 29.0I.I5 & Standort I & $\begin{array}{l}\text { Interview mit } \\
\text { Produktionsmanagerin }\end{array}$ \\
\hline 03.02.I5 & Standort 2 & $\begin{array}{l}\text { Beobachtungsprotokoll: Strickmaschinen- } \\
\text { operateur }\end{array}$ \\
\hline 03.02.15 & Standort 2 & Interview mit CEO \\
\hline 03.02.I5 & Standort 2 & $\begin{array}{l}\text { Interview mit } \\
\text { Produktemanagerin }\end{array}$ \\
\hline 05.02.I5 & Standort 2 & Interview mit Designerin \\
\hline 09.02.I5 & Standort I & Beobachtungsprotokoll: Modenschau \\
\hline 24.02.15 & Standort 2 & Beobachtungsprotokoll: Musterung \\
\hline 09.05.15 & Standort 2 & Beobachtungsprotokoll: Messebesuch \\
\hline 30.06.15 & Standort 2 & $\begin{array}{l}\text { Beobachtungsprotokoll: Musterung/Vorstel- } \\
\text { lung Entwürfe Sommer 20I6 }\end{array}$ \\
\hline & & \\
\hline
\end{tabular}




\begin{tabular}{|c|c|c|}
\hline O6.07.I5 & Standort I & $\begin{array}{l}\text { Beobachtungsprotokoll: Vorstellung Entwürfe } \\
\text { Herbst/Winter } 2016\end{array}$ \\
\hline 05.IO.I5 & Standort I & Beobachtungsprotokoll: zweite Musterung \\
\hline 29.10 .15 & Standort 2 & $\begin{array}{l}\text { Beobachtungsprotokoll: externer Anlass: } \\
\text { Case Study in Gymnasium }\end{array}$ \\
\hline O2.II.I5 & Standort 2 & $\begin{array}{l}\text { Beobachtungsprotokoll: Shop-Verantwort- } \\
\text { lichen-Treffen }\end{array}$ \\
\hline O5.II.I5 & Standort I & Beobachtungsprotokoll: Gegenmuster \\
\hline I7.I2.I5 & Standort 2 & $\begin{array}{l}\text { Beobachtungsprotokoll: Recap mit Designe- } \\
\text { rin }\end{array}$ \\
\hline $\begin{array}{l}\text { II.OI - } \\
05.02 .16\end{array}$ & Standort I & Beobachtungsprotokoll: Shadowing (20 Tage) \\
\hline 07.02 .16 & Standort I & Beobachtungsprotokoll: Fitting, Modenschau \\
\hline 10.02 .16 & Standort I & $\begin{array}{l}\text { Beobachtungsprotokoll: Vorstellung Entwürfe } \\
\text { Frühjahr/Sommer 20I7 }\end{array}$ \\
\hline I8.02.I6 & Standort 2 & $\begin{array}{l}\text { Beobachtungsprotokoll: Recap mit Designe- } \\
\text { rin }\end{array}$ \\
\hline 06.07 .16 & Standort 2 & $\begin{array}{l}\text { Beobachtungsprotokoll: Musterungen, Grafi- } \\
\text { kertreffen }\end{array}$ \\
\hline 06.07 .16 & Standort 2 & $\begin{array}{l}\text { Interview mit Strickmaschinenprogrammie- } \\
\text { rer }\end{array}$ \\
\hline 14.07 .16 & Standort 2 & Beobachtungsprotokoll: Fotoshooting \\
\hline 07.09 .16 & Standort I & $\begin{array}{l}\text { Beobachtungsprotokoll: Übergabe Schnitte } \\
\text { an Couture-Schneiderin }\end{array}$ \\
\hline OI.03.I7 & Standort I & Beobachtungsprotokoll: Fotoshooting \\
\hline 2I.०8.I7 & Standort 2 & $\begin{array}{l}\text { Beobachtungsprotokoll: Kick-off-Meeting } \\
\text { Herbst/Winter 20I7/I8 }\end{array}$ \\
\hline 26.10.17 & Standort 2 & Beobachtungsprotokoll: Fotoshooting \\
\hline
\end{tabular}


Anhang B: Organisation und Darstellung DER MOdEKREATION

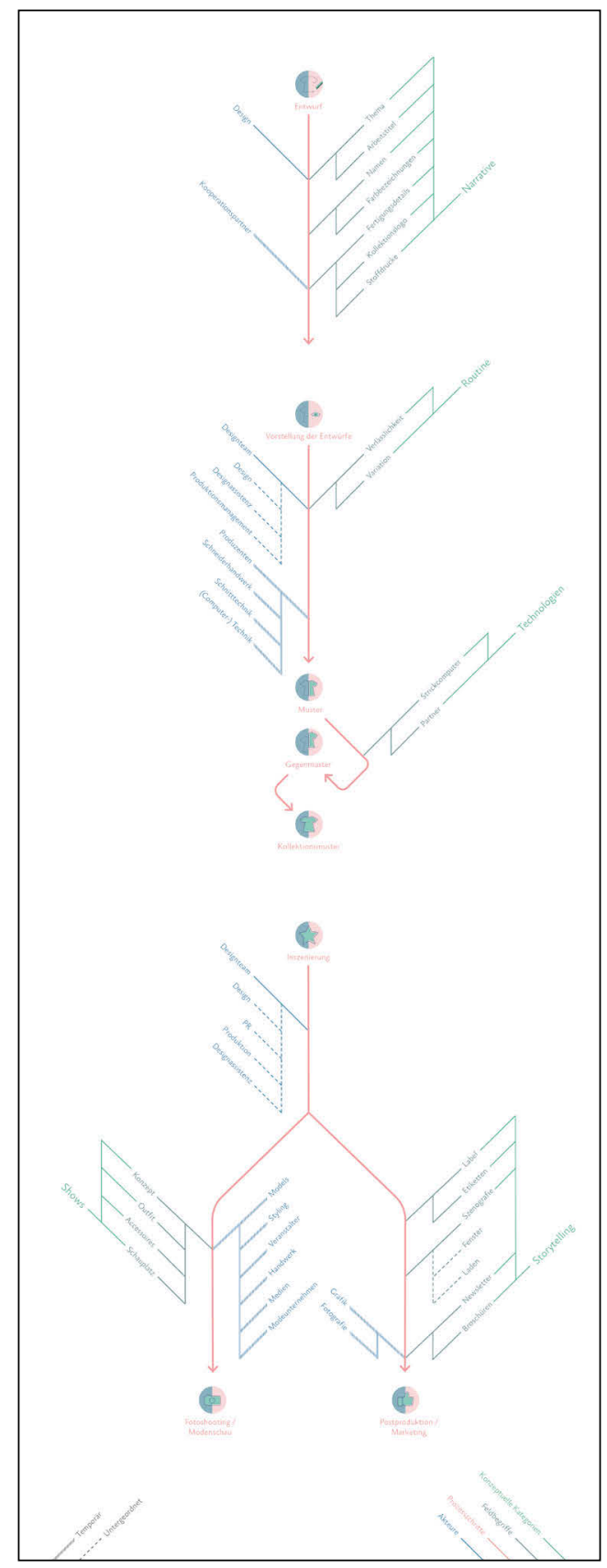




\section{Anhang C: IntervieWLeItFAden}

In der explorativen Phase fanden neun semistrukturierte Interviews mit Mitgliedern der Designteams an ihrem Arbeitsplatz statt.

Einstieg:

Woran orientieren Sie sich/ihr euch?

Wer/was inspiriert Sie/euch?

Wer gibt den Ton an?

Wer ist gut in »Mode machen«? Wieso sind die gut?

Aufgabengebiet/Tätigkeit:

Was ist Ihre/deine Aufgabe?

Beschreibe einen gewöhnlichen Arbeitstag.

Was sind Probleme dabei? Wie gehen Sie diese an/gehst du diese an?

Beschreibe einen außergewöhnlichen Arbeitstag.

Selbstwahrnehmung/Beobachtung im Feld:

Was unterscheidet Sie/euch von anderen Modefirmen? Was ist speziell an Ihnen/euch?

Was macht es wahrscheinlich, dass Ihre/eure Kollektion ankommt?

Wie unterscheiden sich Modefirmen von anderen Organisationen?

Die Transkripte der Interviews wurden mit folgenden Informationen komplettiert: Art des Dokuments, Dauer, Ort und Anschrift, Datum und Uhrzeit, anwesende Personen, Datum Transkript und Dateibenennungslogik. 


\section{Anhang D: Vorlage Feldnotizen}

FELDNOTIZEN DATUM
ORT

NAME, FUNKTION

\begin{tabular}{|l|l|l|}
\hline \multicolumn{2}{|l|}{ SETTING } & \multicolumn{2}{l|}{ Überlegungen } \\
\hline Setting & Anwesende \\
\hline Anwesende: & \\
\hline
\end{tabular}

\section{ERWARTUNGEN EX ANTE}

- Erwartungen

BEOBACHTUNGSPROTOKOLL

\begin{tabular}{|l|l|}
\hline Beobachtung & Überlegungen \\
\hline Beobachtung & Überlegungen \\
\hline
\end{tabular}

ERWARTUNGEN/ FRAGEN EX POST FÜR WEITERE ERHEBUNGEN

- Frage 1

- Frage 2

MATERIAL

Material 1 


\section{Anhang E: Telefoninterviews}

Um einen ersten Eindruck der Verwendung und Assoziationen zum Begriff »Kreativität« in der Mode zu erhalten, wurden Dozierende höherer Fachschulen und Fachhochschulen, die Ausbildungsgängen in Mode- und/oder Textildesign anbieten, kurz telefonisch befragt. Die Antworten haben gezeigt, dass weder von einer einheitlichen Verwendung des Kreativitätsbegriffs noch von homogenen Assoziationen damit gesprochen werden kann. Diese diversen Aussagen wurden zur Grundlage für die weitere Entwicklung des Forschungsdesigns.

Vorstudie Kreativität

Name:

Schule/Institut:

Datum und Zeit des Anrufs:

Was ist Kreativität für Sie?

Wer oder was ist kreativ oder kann es sein?

Wie drückt sich das aus?

Was ist nicht kreativ? 


\section{Dank}

An dieser Stelle danke ich den Menschen ganz herzlich, die mich bei der Entstehung dieses Buches inspiriert, unterstützt und begleitet haben. Im Rahmen der Anstellung als wissenschaftliche Assistentin am Soziologischen Seminar der Universität Luzern hatte ich die Möglichkeit, meinem thematischen Interesse an der Mode zu folgen und daraus eine soziologische Problemstellung zu generieren.

Dieses Buch konnte nur entstehen, weil mir zwei Schweizer Modebetriebe großzügig Zugang und Einsicht in ihren organisatorischen Alltag gewährt haben und weil die Mitarbeitenden bereit waren, ihre Erfahrungen mit mir zu teilen, und sie alle geduldig meine Fragen zur Kreativität und ihrem Organisationsalltag beantworteten.

Raimund Hasse danke ich für die kritischen Hinweise zum Text, die wichtigen Kommentare und Anregungen und die nötige Gewährung großzügiger wissenschaftlicher Freiräume. Sophie Mützel danke ich für ihre Denkanstöße und Kritik, die für mich eine wertvolle Unterstützung dargestellt haben. Beide haben mit ihren Anregungen zur Fokussierung und zu entscheidenden Präzisierungen beigetragen. Schließlich bedanke ich mich bei ihnen für die Begutachtung meiner Arbeit im Rahmen des Promotionsverfahrens an der Universität Luzern. Barbara Czarniawska danke ich für ihre Gastfreundschaft und anregenden Kommentare zum »Methodenkapitel« während meines Aufenthalts am Gothenburg Research Institute.

Darüber hinaus möchte ich mich bei meinen Kolleginnen und Kollegen bedanken, die Teile der Arbeit bei unterschiedlichen Gelegenheiten mit mir diskutiert, wertvolle Anregungen gegeben und/oder durch ihre kritische Lektüre zur Verbesserung des Manuskripts beigetragen haben: Hannah Mormann, Roman Gibel, Nadine Arnold, Eva Passarge, Lea Fünfschilling, Sven Kette, Philippe Saner, Markus Unternährer, Judith Tonner und Anne Beutter. Ihre konstruktiven Kommentare und ermutigenden Worte haben nicht nur dabei geholfen, den Fokus nicht zu verlieren, sondern auch maßgebend zum Spaß an wissenschaftlicher Arbeit beigetragen. Isabelle Haffter und Leonie Häsler danke ich für ihre moralische Unterstützung. Isabel Christen hat mir mit viel Geduld bei der Erstellung der Infografiken in diesem Buch geholfen. 
Meinen Eltern und meinen Brüdern danke ich für die bedingungslose Unterstützung meines Werdegangs, ihren Ansporn und die vielen, manchmal hitzigen, aber vor allem motivierenden Gespräche. David Schneiter danke ich von ganzem Herzen für seinen Rückhalt, seine Ermutigungen und seine Liebe. 


\section{Soziologie}

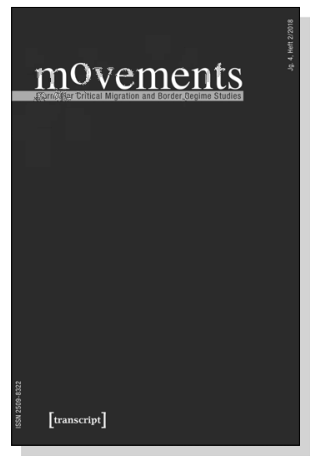

Juliane Karakayali, Bernd Kasparek (Hg.)

movements.

Journal for Critical Migration and Border Regime Studies

Jg. 4, Heft 2/2018

Februar 2019, 246 S., kart.

24,99 €(DE), 978-3-8376-4474-6

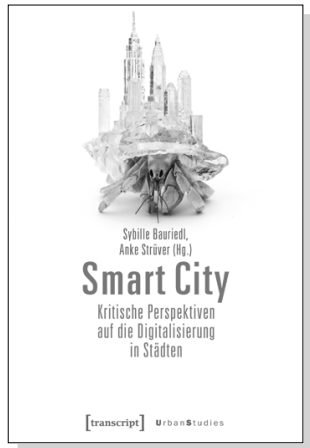

Sybille Bauriedl, Anke Strüver (Hg.)

\section{Smart City -}

Kritische Perspektiven auf die Digitalisierung in Städten

2018, 364 S., kart.

29,99€ (DE), 978-3-8376-4336-7

E-Book: 26,99 € (DE), ISBN 978-3-8394-4336-1

EPUB: $26,99 €(D E)$, ISBN 978-3-7328-4336-7

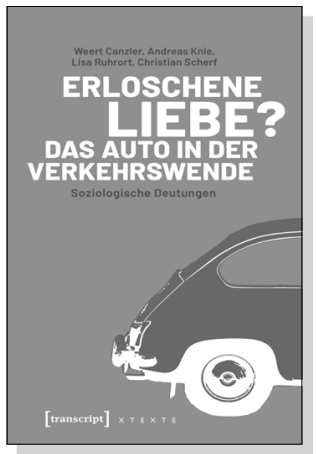

Weert Canzler, Andreas Knie, Lisa Ruhrort, Christian Scherf

\section{Erloschene Liebe?}

Das Auto in der Verkehrswende

Soziologische Deutungen

2018, 174 S., kart., zahlr. Abb.

$19,99 €(D E), 978-3-8376-4568-2$

E-Book: $17,99 €$ (DE), ISBN 978-3-8394-4568-6

EPUB: $17,99 €(D E)$, ISBN 978-3-7328-4568-2 


\section{Soziologie}

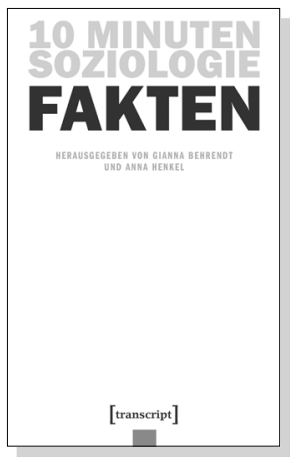

Gianna Behrendt, Anna Henkel (Hg.)

10 Minuten Soziologie: Fakten

2018, 166 S., kart.

$16,99 €(D E), 978-3-8376-4362-6$

E-Book: 14,99 € (DE), ISBN 978-3-8394-4362-0

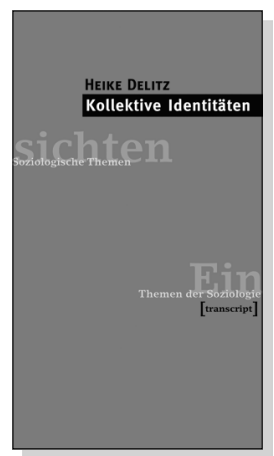

Heike Delitz

Kollektive Identitäten

2018, 160 S., kart.

$14,99 €(D E), 978-3-8376-3724-3$

E-Book: 12,99 € (DE), ISBN 978-3-8394-3724-7

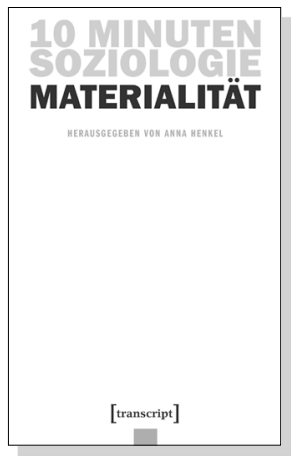

Anna Henkel (Hg.)

10 Minuten Soziologie: Materialität

2018, 122 S., kart.

$15,99 €(D E), 978-3-8376-4073-1$

E-Book: 13,99 €(DE), ISBN 978-3-8394-4073-5 